\title{
Multilevel Panel Method for Wind Turbine Rotor Flow Simulations
}

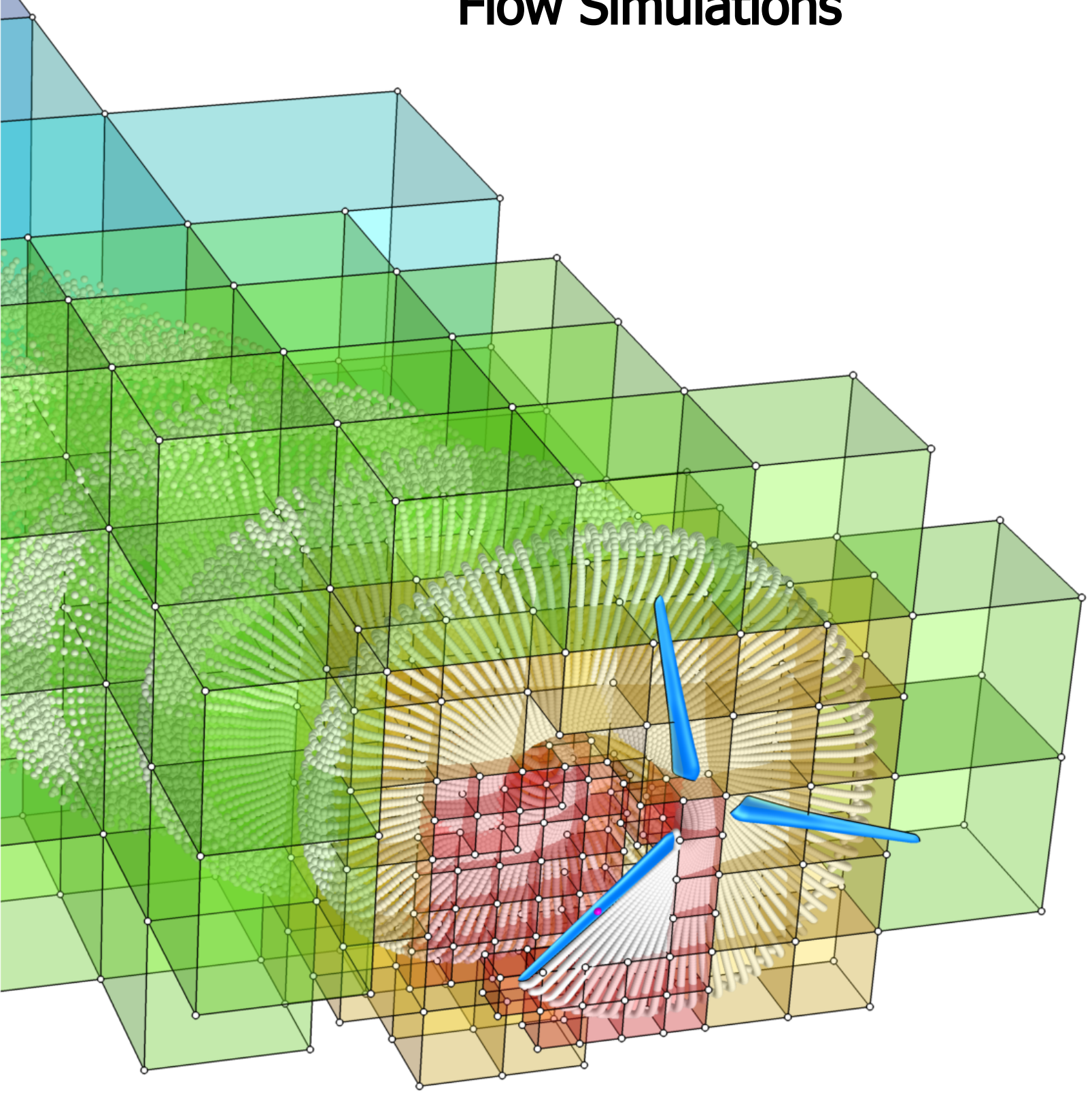


Multilevel Panel Method FOR Wind Turbine Rotor Flow Simulations 



\section{MULTILEVEL PANEL METHOD \\ FOR WIND TURBINE ROTOR \\ FLOW SIMULATIONS}

PROEFSCHRIFT

ter verkrijging van

de graad van doctor aan de Universiteit Twente, op gezag van de rector magnificus, prof.dr. T.T.M. Palstra,

volgens besluit van het College voor Promoties in het openbaar te verdedigen

op woensdag 14 december 2016 om 16:45 uur

door

Arne van Garrel

geboren op 18 februari 1965

te Amsterdam, Nederland 
Dit proefschrift is goedgekeurd door de promotoren:

Prof.dr.ir. H.W.M. Hoeijmakers,

Prof.dr.ir. C.H. Venner.

Samenstelling promotiecommissie:

Prof.dr. G.P.M.R. Dewulf

Prof.dr.ir. H.W.M. Hoeijmakers

Prof.dr.ir. C.H. Venner

Prof.dr.ir. A. de Boer

Prof.dr.ir. J.J.W. van der Vegt

Dr.ir. C.J. Simão Ferreira

Prof.ir. H. Snel

Prof.dr. M. Drela
Universiteit Twente, voorzitter

Universiteit Twente, promotor

Universiteit Twente, promotor

Universiteit Twente

Universiteit Twente

Technische Universiteit Delft

Instituto Tecnológico de Costa Rica

Massachusetts Institute of Technology

Multilevel Panel Method for Wind Turbine Rotor Flow Simulations Thesis University of Twente, Enschede, The Netherlands

Copyright (c) 2016 by A. van Garrel.

ISBN: 978-90-365-4224-1

DOI: $10.3990 / 1.9789036542241$

http://dx.doi.org/10.3990/1.9789036542241

Cover: Multilevel boxes used to determine the influence of the wake of a rotor blade at $30^{\circ}$ yaw at a point on the blade surface.

Gedrukt door CPI - Koninklijke Wöhrmann - Zutphen. 


\section{Summary}

The ongoing trend in wind turbine development is towards larger rotors because of the resulting lower cost of energy. These large rotors lead to relatively flexible structures that are more susceptible to the unsteady aerodynamic loading occurring in normal operating conditions. An accurate prediction of these loadings is important for the design of an economically viable and technically reliable wind turbine. Simulation methods of wind turbine aerodynamics currently in use mainly fall into two categories: the first is the group of traditional low-fidelity engineering models and the second is the group of computationally expensive CFD methods based on the Navier-Stokes equations.

For an engineering environment the search is for "medium fidelity" wind turbine simulation methods that bridge the gap between the computationally inexpensive low-fidelity methods and the computationally expensive CFD methods. The ultimate goal is a balanced mixture of higher accuracy of the representation of the physics and shorter simulation times for wind turbine aerodynamics simulation methods. This can be found in the combination of the theories for panel methods, integral boundary layer methods, strong viscous-inviscid coupling, and fluid structure interaction.

The present study focuses on the development of the theory and the practical implementation of a fast multilevel integral transform in a computer program. We utilize this multilevel scheme in a low-order panel method. It is demonstrated that for the simulation of the wake flow of wind turbine rotors the computational burden is reduced from $\mathcal{O}\left(N^{2}\right)$ for a conventional panel method to $\mathcal{O}(N)$ for the present method. This implies that the computational effort is reduced to grow linearly with problem size $N$, with $N$ the number of panels.

We consider the unsteady, incompressible flow around wind turbine rotors and assume the effects of viscosity to be confined to infinitesimal thin boundary layers and wake regions and assume irrotational flow elsewhere. These assumptions allows us to reduce the flow problem to the problem of solving the Laplace equation for a (scalar) velocity potential function. This makes it possible to reformulate the problem as an integral equation over the surface of the rotor and the wakes that emanate from the trailing edges of the rotating wind turbine blades.

The mathematical model is discretized in the form of a low-order panel 
method. The implementation of the panel method is verified by considering the flow over a stationary ellipsoid in a uniform onset velocity field, the flow over a rotating ellipsoid in a fluid at rest, and by considering the flow over a high aspect ratio wing with elliptic planform with as cross-section a von Kármán-Trefftz airfoil. For the first two test cases the numerical results are compared with analytical solutions. The error in the velocity potential is shown to be $\mathcal{O}\left(h^{2}\right)$, with $h$ a characteristic panel size. The third test case uses the analytical solution for the 2D von Kármán-Trefftz airfoil and the numerical results show the order of accuracy in the pressure distribution to be close to $\mathcal{O}(h)$. The computational cost of this conventional panel method is shown to be growing quadratically with the number of panels.

In the present study a fast multilevel integral transform method has been developed that builds upon the multilevel multi-integration concept of Brandt and Lubrecht who approximated the kernel function in the integrand with a polynomial. The new Multi-Level Multi-Integration Cluster (MLMIC) method is capable of handling highly irregular wake sheets that occur in the wake of the wind turbine. The implementation of the MLMIC method is first verified by considering clouds of point singularities representing the dipole and the source distributions in the panel method. An increasing number of points $N$ is accompanied by an equally fast increasing length of the domain, which mimics the behavior of a wind turbine wake extending downstream in time. The error in the potential function is shown to be controlled by the order of the polynomial interpolation. The computational time is shown to increase as $\mathcal{O}(N)$. In a second verification test the solid angle at points interior to a configuration is considered, for which the numerical results are compared to the exact solution. The error in the solid angle for increasing number of panels is shown to be decreasing, up to a number of panels dictated by the polynomial order used in the MLMIC method, at the same rate as the conventional panel method.

As a validation test the panel method, combined with the MLMIC scheme for the deformation of the wake, is applied to the MEXICO wind tunnel experiment. The numerical results show good agreement with the experimentally obtained pressure distributions at 5 blade sections of this model wind turbine as well as the results of a state-of-the-art solution method for the Reynolds-averaged Navier-Stokes equations. Compared to a conventional panel method in these simulations the MLMIC scheme reduced the computation time for the wake deformation by a factor 150 .

This thesis ends with some concluding remarks and an outlook on future developments that are now feasible with the reduction of computation time achieved by the new MLMIC method. The current work repositions panel 
methods as excellent computational design tool for wind turbine blades. 


\section{Samenvatting}

De aanhoudende trend naar grotere rotoren in de ontwikkeling van windturbines wordt ingegeven door de daaruit voortvloeiende lagere energie kosten. Deze grote rotoren leiden tot relatief flexibele constructies die gevoeliger zijn voor de tijdafhankelijke aerodynamische krachten die optreden bij normale bedrijf omstandigheden van windturbines. Een nauwkeurige voorspelling van deze belastingen op de constructie is belangrijk voor het ontwerp van een economisch haalbare en technisch betrouwbare windturbine. De meest gangbare methoden die momenteel in gebruik zijn voor de numerieke simulatie van windturbine aerodynamica vallen in twee categorieën: de eerste is de groep van traditionele "low-fidelity" engineering modellen en de tweede is de groep van rekenkundig dure CFD-methoden voor het oplossen van de Navier-Stokes vergelijkingen.

Voor een engineering omgeving is het zoeken naar "medium fidelity" windturbine simulatie methoden die de kloof overbruggen tussen de rekenkundig goedkope low-fidelity methoden en de dure CFD methoden. Het uiteindelijke doel is een dergelijke evenwichtige mix van korte rekentijden en hogere nauwkeurigheid voor de representatie van de fysica. De verwezenlijking van dit ultieme doel is te vinden in de combinatie van de theorieën voor de panelen methoden, integraal grenslaag methoden, sterke viskeuze - nietviskeuze koppeling, en de interactie tussen aerodynamische krachten en de dynamica van de constructie.

Het huidige onderzoek richt zich op de ontwikkeling van de theorie en de praktische implementatie van een snelle multilevel integraal transformatie in een reken methode. We maken gebruik van deze multilevel methode in een lage-orde panelen methode. We tonen aan dat voor de simulatie van het zog achter de windturbine rotor de rekenlast wordt verlaagd van $\mathcal{O}\left(N^{2}\right)$ voor een conventionele panelen methode naar $\mathcal{O}(N)$ voor de nieuwe multilevel aanpak. Dit impliceert dat de rekentijd is gereduceerd tot een lineair met het aantal panelen $N$ groeiende functie.

We beschouwen tijdafhankeljke, onsamendrukbare stromingen rond windturbine rotoren en nemen aan dat de effecten van de viscositeit beperkt zijn tot oneindig dunne grenslagen en zog oppervlakken, en veronderstellen een rotatievrije stroming in de rest van de ruimte. Met deze aannames kunnen we het stromingsprobleem reduceren tot het oplossen van de Laplace vergelijking voor een (scalaire) snelheidspotentiaal. Dit maakt het mogelijk om het probleem te herformuleren als integraal vergelijking over het opper- 
vlak van de bladen van de rotor en de zogoppervlakken die zich ontwikkelen vanaf de achterrand van de roterende windturbinebladen.

Het rekenmodel is gediscretiseerd in de vorm van een lagere orde panelen methode. De implementatie van de panelen methode is geverifieerd aan de hand van een drietal testgevallen. Het eerste testgeval richt zich op de stroming over een stilstaande ellipsoïde in een uniform wind snelheidsveld. De tweede test behandelt de stroming over een roterende ellipsoïde in stilstaande lucht. Het derde testgeval beschouwt de stroming over een vleugel. Deze vleugel heeft grote slankheid en een elliptische planform met als dwarsdoorsnede een Von Kármán-Trefftz profiel. Voor de eerste twee testgevallen worden de numerieke resultaten vergeleken met analytische oplossingen en wordt aangetoond dat de fout in de snelheidspotentiaal $\mathcal{O}\left(h^{2}\right)$ is, met $h$ een karakteristieke paneelgrootte. Het derde testgeval maakt gebruik van de analytische oplossing voor de stroming om het 2D Von Kármán-Trefftz vleugelprofiel en de numerieke resultaten tonen aan dat de nauwkeurigheid in de drukverdeling in de buurt van $\mathcal{O}(h)$ ligt. De rekentijd van deze conventionele panelen methode groeit kwadratisch met het aantal panelen.

In de huidige studie is een snelle multilevel integraal transformatie methode ontwikkeld die voortbouwt op het multilevel multi-integratie concept van Brandt en Lubrecht waarin de kernfunctie in de integrand met een polynoom wordt benaderd. De nieuwe Multi-Level Multi-Integration Cluster (MLMIC) methode is geschikt voor zeer onregelmatige oppervlakken zoals die zich voordoen in het zog van de windturbine. De implementatie van de MLMIC methode wordt eerst geverifieerd door de dipool en de bron verdelingen in de panelen methode te representeren als wolken van punt singulariteiten. De toename van het aantal punten $N$ gaat gepaard met een even snel in lengterichting toenemende domein grootte, hetgeen het gedrag nabootst van een windturbine zog dat zich in de tijd stroomafwaarts uitbreidt. De fout in de snelheidspotentiaal functie kan worden gestuurd met de keuze voor de orde van het interpolatie polynoom. Er wordt aangetoond dat de rekentijd toeneemt met $\mathcal{O}(N)$. In een tweede verificatie test beschouwen we de ruimtehoek in punten in het inwendige van een object. De numerieke resultaten worden vergeleken met de exacte oplossing. De fout in de ruimtehoek voor toenemend aantal panelen neemt af met dezelfde snelheid als voor de conventionele panelen methode, dit tot een aantal panelen dat wordt bepaald door de orde van het interpolatie polynoom in de MLMIC methode.

Als validatie test voor de panelen methode gecombineerd met de MLMIC methode voor de vervorming van het rotor zog beschouwen we resultaten van het MEXICO windtunnel experiment. De huidige numerieke resultaten 
tonen een goede overeenkomst met de experimenteel verkregen drukverdelingen langs 5 bladsecties van de model windturbine, zowel als met de resultaten van een state-of-the-art numerieke simulatie methode voor de Reynoldsgemiddelde Navier-Stokes vergelijkingen. Vergeleken met een conventionele panelen methode reduceert de MLMIC methode voor deze testgevallen de rekentijd voor de vervorming van het zog met een factor 150 .

Dit proefschrift eindigt met enkele concluderende opmerkingen en een vooruitblik op de mogelijke toekomstige ontwikkelingen die binnen bereik zijn gekomen door de korte rekentijden benodigd voor de nieuwe MLMIC methode. Het huidige werk herpositioneerd panelen methoden als uitstekend eerste numeriek ontwerpgereedschap voor windturbine bladen. 


\section{Contents}

Summary

Samenvatting vii

Contents $\quad x i$

$\begin{array}{ll}\text { Nomenclature } & \text { Xv }\end{array}$

1 Introduction 1

1.1 Wind Energy . . . . . . . . . . . . . . . 1

1.2 Wind Turbine Aerodynamics .............. 3

1.3 Challenges.................... 7

1.4 Thesis Outline ................. 10

2 Potential Flow Model 13

2.1 Introduction . . . . . . . . . . . . . . . . . 13

2.2 Governing Equations . . . . . . . . . . . . . . . 14

2.3 Boundary Integral Equation . . . . . . . . . . . . . 16

2.4 Boundary Conditions . . . . . . . . . . . . . . 18

2.4.1 Introduction . . . . . . . . . . . . . 18

2.4 .2 Body Surface . . . . . . . . . . . . 18

2.4.3 Wake Surface ............... 22

2.5 Pressure, Forces, and Moments . . . . . . . . . . . . 24

3 Low Order Panel Method 27

3.1 Introduction . . . . . . . . . . . . . . . 27

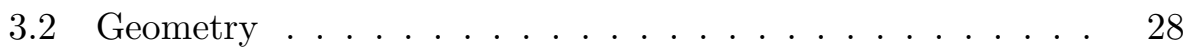

3.2.1 Solid Body Transformations . . . . . . . . . . 30

3.3 Aerodynamic Influence Coefficients . . . . . . . . . . . . . 32 
3.3.1 Dipole Potential and Velocity . . . . . . . . . . . 34

3.3.2 Source Potential and Velocity . . . . . . . . . . . . 37

3.4 Post-processing . . . . . . . . . . . . . . . . . 37

3.4 .1 Velocity Vector Field . . . . . . . . . . . . . . 37

3.4.2 Aerodynamic Forces and Moments . . . . . . . . 39

3.5 Verification . . . . . . . . . . . . . . 40

3.5 .1 Geometry . . . . . . . . . . . . . . . 41

3.5 .2 Solid Angle . . . . . . . . . . . . . . . . 42

3.5.3 Ellipsoid in Uniform Onset Flow . . . . . . . . . 48

3.5.4 Rotating Ellipsoid in Fluid at Rest . . . . . . . . . 51

3.5.5 Elliptic Wing with von Kármán-Trefftz Airfoil . . . 55

4 Fast Cluster Multilevel Algorithm $\quad 59$

4.1 Introduction . . . . . . . . . . . . . . . . 59

4.2 Multi-Level Multi-Integration Cluster Scheme . . . . . . . . 61

4.2 .1 Smooth Kernels . . . . . . . . . . . . . . . . . . 63

$4.2 .2 \quad$ Singular Kernels . . . . . . . . . . . . . . . . . . . 68

4.3 Higher Dimensions . . . . . . . . . . . . . . . . . 73

4.3 .1 Singular Kernels . . . . . . . . . . . . . . . . 74

4.3.2 Surface Singularity Distributions . . . . . . . . 76

4.3.3 Vector (Component) Kernels . . . . . . . . . . 78

4.4 Implementation . . . . . . . . . . . . . . . . . . . . 79

4.5 MLMIC Verification . . . . . . . . . . . . . 81

4.5.1 Source and Dipole Potentials . . . . . . . . . 83

4.5 .2 Solid Angle Tests . . . . . . . . . . . . . . . . 90

4.6 Related Work . . . . . . . . . . . . . . . . . . . . . . 93

4.6.1 Fast Multipole Method . . . . . . . . . . . . 93

4.6.2 Panel Clustering Method . . . . . . . . . . . . . 95

4.6.3 Multi-Level Multi Integration . . . . . . . . . . . . 96

4.6.4 Precorrected-FFT Method . . . . . . . . . . . 99 
5 Application $\quad 101$

5.1 MEXICO experiment ................ 101

6 Conclusions and Outlook 113

6.1 Conclusions .................... 113

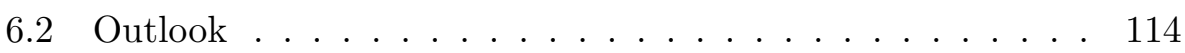

$\begin{array}{lr}\text { References } & 117\end{array}$

$\begin{array}{lr}\text { A Mathematical Compendium } & 127\end{array}$

B Conservation Laws $\quad 131$

B.1 Mass Conservation . . . . . . . . . . . . . . . 131

B.2 Momentum Conservation ............... 133

B.3 Energy Conservation . . . . . . . . . . . . . 136

C Helmholtz Decomposition $\quad 141$

D Rotational Onset Flow 143

$\begin{array}{ll}\text { E Boundary Integral Equation } & 147\end{array}$

F Barycentric Lagrange Interpolation 153

G Tri-Axial Ellipsoids $\quad 157$

G.1 Ellipsoid Geometry . . . . . . . . . . . . . . . 158

G.2 Ellipsoid in Uniform Onset Flow . . . . . . . . . . . . . 159

G.3 Rotating Ellipsoid in Fluid at Rest . . . . . . . . . . . . . . 160

G.4 Flow Solutions for a Two-Dimensional Ellipse . . . . . . . 163

$\begin{array}{lr}\text { Acknowledgements } & 169\end{array}$

$\begin{array}{lr}\text { About the Author } & 171\end{array}$ 


\section{Nomenclature}

\section{Dimensions}

[K] kelvin temperature

[kg] kilogram mass

[m] meter length

$[\mathrm{s}]$ second time

[N] newton force $\left[\mathrm{kg} \cdot \mathrm{m} \cdot \mathrm{s}^{-2}\right]$

$[\mathrm{J}]$ joule energy, work, heat $[\mathrm{N} \cdot \mathrm{m}]$

$[\mathrm{W}]$ watt power $\left[\mathrm{J} \cdot \mathrm{s}^{-1}\right]$

[rad $]$ radian angle $\left[\mathrm{m} \cdot \mathrm{m}^{-1}\right]$

[-] dimensionless

$[\cdots]$ dimension from context

\section{Acronyms}

BEM Blade Element Momentum

CFD Computational Fluid Dynamics

CPU Central Processing Unit

FMM Fast Multipole Method

FSI Fluid-Structure Interaction

GMRES Generalized Minimal RESidual

IBL Integral Boundary Layer

KT Von Kármán - Trefftz

LES Large Eddy Simulation

MLMI Multi-Level Multi-Integration

MLMIC Multi-Level Multi-Integration Cluster

RaNS Reynolds-averaged Navier-Stokes

RPM Revolutions Per Minute

VII Viscous-Inviscid Interaction

2D Two-Dimensional

3D Three-Dimensional 


\section{Roman symbols}

\begin{tabular}{|c|c|c|}
\hline$a, b, c$ & {$[\mathrm{~m}]$} & ellipsoid semi-axes \\
\hline $\mathcal{B}$ & {$[-]$} & box \\
\hline$C_{p}$ & {$[-]$} & pressure coefficient \\
\hline$c$ & {$[\mathrm{~m}]$} & chord length \\
\hline$c$ & {$[-]$} & constant, number of boxes \\
\hline$c, C$ & {$\left[\mathrm{~m}^{2} \cdot \mathrm{s}^{-2}\right]$} & Bernoulli constant \\
\hline$c_{p}$ & {$\left[\mathrm{~J} \cdot \mathrm{kg}^{-1} \cdot \mathrm{K}^{-1}\right]$} & specific heat capacity at constant pressure \\
\hline$c_{v}$ & {$\left[\mathrm{~J} \cdot \mathrm{kg}^{-1} \cdot \mathrm{K}^{-1}\right]$} & specific heat capacity at constant volume \\
\hline$d$ & {$[-]$} & space dimension, relative distance \\
\hline$E$ & {$\left[\mathrm{~J} \cdot \mathrm{kg}^{-1}\right]$} & total specific energy \\
\hline$e$ & {$\left[\mathrm{~J} \cdot \mathrm{kg}^{-1}\right]$} & internal specific energy \\
\hline$H$ & {$\left[\mathrm{~J} \cdot \mathrm{kg}^{-1}\right]$} & total specific enthalpy \\
\hline$h$ & {$\left[\mathrm{~J} \cdot \mathrm{kg}^{-1}\right]$} & specific enthalpy \\
\hline$h$ & {$[\mathrm{~m}]$} & box size, panel size \\
\hline$i, j$ & {$[-]$} & index numbers \\
\hline$J$ & {$\left[\mathrm{~m}^{2}\right]$} & surface transformation Jacobian \\
\hline$K$ & {$[\cdots]$} & kernel function \\
\hline$L$ & {$[-]$} & Lagrange polynomial \\
\hline $\mathrm{L}$ & {$[\mathrm{m}]$} & length \\
\hline$l$ & {$[\mathrm{~m}]$} & line element \\
\hline $\mathrm{Ma}$ & {$[-]$} & Mach number \\
\hline$N$ & {$[-]$} & problem size \\
\hline$n$ & {$[-]$} & number of elements \\
\hline$P$ & {$[\mathrm{~W}]$} & power \\
\hline$p$ & {$[-]$} & polynomial order, i.e. degree +1 \\
\hline$p$ & {$\left[\mathrm{~N} \cdot \mathrm{m}^{-2}\right]$} & pressure \\
\hline$Q$ & {$\left[\mathrm{~J} \cdot \mathrm{m}^{-3}\right]$} & heat \\
\hline $\operatorname{Re}$ & {$[-]$} & Reynolds number \\
\hline$r$ & {$[\mathrm{~m}]$} & distance \\
\hline$S$ & {$\left[\mathrm{~m}^{2}\right]$} & surface \\
\hline$T$ & {$[\mathrm{~K}]$} & temperature \\
\hline$t$ & {$[\mathrm{~s}]$} & time \\
\hline$t_{1}, t_{2}, t_{3}$ & {$[\mathrm{~m}]$} & translation vector components \\
\hline$U$ & {$\left[\mathrm{~m} \cdot \mathrm{s}^{-1}\right]$} & velocity \\
\hline$V$ & {$\left[\mathrm{~m}^{3}\right]$} & volume \\
\hline$v$ & {$\left[\mathrm{~m} \cdot \mathrm{s}^{-1}\right]$} & velocity \\
\hline$v_{1}, v_{2}, v_{3}$ & {$[-]$} & rotation axis components \\
\hline$\partial S$ & {$[\mathrm{~m}]$} & surface boundary \\
\hline$\partial V$ & {$\left[\mathrm{~m}^{2}\right]$} & volume boundary \\
\hline$x, y, z$ & {$[\mathrm{~m}]$} & Cartesian coordinates \\
\hline
\end{tabular}




\section{Greek symbols}

\begin{tabular}{|c|c|}
\hline$\alpha$ & {$[\mathrm{rad}]$} \\
\hline$\alpha_{0}, \beta_{0}, \gamma_{0}$ & {$[-]$} \\
\hline$\gamma$ & {$[-]$} \\
\hline$\delta_{i k}$ & {$[-]$} \\
\hline$\delta()$ & {$[\cdots$} \\
\hline$\delta, \Delta$ & {$[\mathrm{m}]$} \\
\hline$\Delta$ & {$[-]$} \\
\hline$\partial \Omega$ & $\cdots]$ \\
\hline$\epsilon$ & $\cdots$ \\
\hline$\varepsilon$ & {$[\mathrm{m}]$} \\
\hline$\Phi$ & {$\left[\mathrm{m}^{2} \cdot \mathrm{s}^{-1}\right]$} \\
\hline$\varphi$ & {$\left[\mathrm{m}^{2} \cdot \mathrm{s}^{-1}\right]$} \\
\hline$\Gamma$ & {$\left[\mathrm{m}^{2} \cdot \mathrm{s}^{-1}\right]$} \\
\hline$\kappa$ & {$\left[\mathrm{W} \cdot \mathrm{m}^{-1} \cdot \mathrm{K}^{-1}\right]$} \\
\hline$\lambda$ & {$[-]$} \\
\hline$\mu$ & {$\left[\mathrm{kg} \cdot \mathrm{m}^{-1} \cdot \mathrm{s}^{-1}\right]$} \\
\hline$\mu$ & {$\left[\mathrm{m}^{2} \cdot \mathrm{s}^{-1}\right]$} \\
\hline$\nu$ & {$\left[\mathrm{m}^{2} \cdot \mathrm{s}^{-1}\right]$} \\
\hline$\Omega$ & {$[\cdots]$} \\
\hline$\Psi$ & {$[\cdots]$} \\
\hline$\psi$ & {$[\cdots]$} \\
\hline$\rho$ & {$\left[\mathrm{kg} \cdot \mathrm{m}^{-3}\right]$} \\
\hline$\sigma$ & {$\left[\mathrm{m} \cdot \mathrm{s}^{-1}\right]$} \\
\hline$\sigma_{v}$ & {$\left[\mathrm{~s}^{-1}\right]$} \\
\hline$\theta$ & {$[\mathrm{rad}]$} \\
\hline$\vartheta$ & {$[-]$} \\
\hline$\xi, \eta$ & {$[-]$} \\
\hline$\xi_{0}, \eta_{0}, \zeta_{0}$ & {$[-]$} \\
\hline
\end{tabular}

angle of attack

elliptic integral values

specific heat ratio

Kronecker delta

Dirac delta function

length scales

difference

space boundary

error

infinitesimal distance

velocity potential

velocity perturbation potential

vortex strength

thermal conductivity

local speed ratio, scaling factor, wing aspect ratio

dynamic viscosity coefficient

dipole strength

kinematic viscosity

space

scalar field

scalar field

mass density

source strength

volume source distribution

rotation angle

solid angle

non-orthogonal coordinate system directions

elliptic integral values 


\section{Vectors, matrices, and tensors}

\begin{tabular}{|c|c|c|}
\hline$\vec{a}$ & {$[\cdots]$} & general vector field \\
\hline$\vec{\alpha}$ & {$[\cdots]$} & solenoidal vector field, i.e. $\nabla \cdot \vec{\alpha}=0$ \\
\hline$\vec{F}, \vec{f}$ & {$[\mathrm{~N}]$} & force vector \\
\hline$\vec{f}$ & {$\left[\mathrm{~m} \cdot \mathrm{s}^{-2}\right]$} & force vector per unit mass \\
\hline$\vec{g}$ & {$\left[\mathrm{~m} \cdot \mathrm{s}^{-2}\right]$} & gravitational acceleration vector \\
\hline$\vec{\gamma}$ & {$\left[\mathrm{m} \cdot \mathrm{s}^{-1}\right]$} & surface vorticity distribution \\
\hline$\vec{h}$ & {$[\cdots]$} & harmonic vector field, i.e. $\nabla \cdot \vec{h}=0, \nabla \times \vec{h}=0$ \\
\hline$\vec{l}$ & {$[\mathrm{~m}]$} & line element \\
\hline$\vec{M}, \vec{m}$ & {$[\mathrm{~N} \cdot \mathrm{m}]$} & moment vector \\
\hline $\bar{n}$ & {$[-]$} & unit surface normal vector \\
\hline$\vec{\Omega}$ & {$\left[\mathrm{rad} \cdot \mathrm{s}^{-1}\right]$} & angular velocity vector \\
\hline$\vec{\omega}_{v}$ & {$\left[\mathrm{~s}^{-1}\right]$} & volume vorticity distribution \\
\hline$\vec{q}$ & {$\left[\mathrm{~W} \cdot \mathrm{m}^{-2}\right]$} & heat flux density \\
\hline $\mathbf{R}$ & {$[-]$} & rotation matrix \\
\hline$\vec{r}$ & {$[\mathrm{~m}]$} & displacement vector \\
\hline $\mathbf{T}$ & {$[-]$} & translation matrix \\
\hline$\vec{\tau}$ & {$\left[\mathrm{N} \cdot \mathrm{m}^{-2}\right]$} & viscous stress vector \\
\hline$\overline{\bar{\tau}}$ & {$\left[\mathrm{N} \cdot \mathrm{m}^{-2}\right]$} & viscous stress tensor \\
\hline$\vec{u}$ & {$\left[\mathrm{~m} \cdot \mathrm{s}^{-1}\right]$} & velocity vector \\
\hline $\bar{v}$ & {$[-]$} & rotation axis direction \\
\hline$\vec{x}$ & {$[\mathrm{~m}]$} & point location \\
\hline$\vec{y}$ & {$[\mathrm{~m}]$} & point location \\
\hline
\end{tabular}




\section{Subscripts and superscripts}

()$_{\infty} \quad$ onset flow value

()$_{e} \quad$ value at ellipsoid surface

()$_{\text {ff }} \quad$ value in far field

()$_{\varepsilon} \quad$ infinitesimal size

()$_{\eta} \quad$ component in $\eta$-direction

()$^{h} \quad$ value at finer grid level

()$^{H} \quad$ value at coarser grid level

()$^{I} \quad$ coarser receiver grid nodes

()$^{J} \quad$ coarser source grid nodes

( ) I coarser receiver grid nodes

( ) $J \quad$ coarser source grid nodes

()$_{i} \quad$ receiver indices

()$_{j} \quad$ source indices

()$_{\mu} \quad$ value from dipole singularity

()$_{n} \quad$ value in surface normal direction

( ) nf value in near field

()$^{p} \quad$ principal value or finite part integral value

( ) ref reference value

( ) rel value in moving frame of reference

()$_{S} \quad$ surface component

()$_{\sigma} \quad$ value from source singularity

()$_{v} \quad$ value from volume source distribution

()$^{\mathrm{T}} \quad$ transpose operator

()$_{t} \quad$ value in surface tangential direction

()$_{t e} \quad$ value at the trailing edge

()$_{v} \quad$ value in volume

()$_{w} \quad$ value at the wake surface

( ) $\omega_{v} \quad$ value from volume vorticity distribution

()$_{m}$. value at surface fluid side

()$\cdot k \quad$ value at surface fictitious side

()$_{\xi} \quad$ component in $\xi$-direction

()$_{x} \quad$ component in $x$-direction

( ) $y \quad$ component in $y$-direction

()$_{z} \quad$ component in $z$-direction

( ) average value

( ) extended field

$\left(^{*}\right)$ time derivative

$\rightarrow$ vector

$\left(^{-}\right) \quad$ vector of unit length 


\section{Introduction}

\subsection{Wind Energy}

Wind energy has been an important part of the Netherlands heritage. Probably introduced in the Netherlands from north-western France or from Flanders the windmill was quickly recognized as a very powerful tool to do work that was previously performed by animal or human labor. The flat and open Dutch countryside with its good wind climate must have been an important factor in the widespread adaptation of wind mills as well as the multitude of waterways over which the raw materials could be supplied. There were mills for grinding grain, oil seeds, shells, hemp, bones, etc. Another important usage for the wind mill was in pumping lakes dry and reclaiming land from the sea after dikes had been raised around areas that would flood during high tide. The benefit of this is obvious, the reclaimed land could be used for agriculture while increasing the accessibility of the existing land and decreasing health problems associated with for example rats, fungi, and mosquitoes.

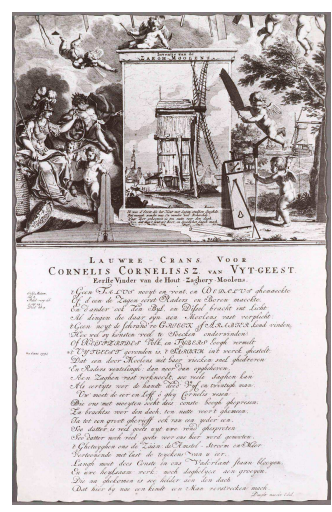

(a) Patent.

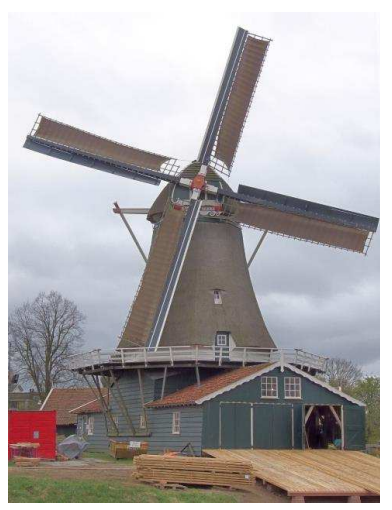

(b) Sawmill.

Figure 1.1: Dutch Sawmill Patent for Cornelis Corneliszoon.[27]

The importance of the invention of the wind powered sawmill in 1594 by Cornelis Corneliszoon van Uitgeest can not be overstated. It made the conversion of $\log$ timber into planks 30 times faster than before. This made the construction of wood houses much more affordable and made the construction of a massive fleet of ships possible. The sawmill thus formed part 
of the basis of the "Dutch Golden Age", the period that spanned roughly from 1600 to 1700 in which the Dutch Republic was the foremost maritime and economic power in the world [115]. The increase in wealth had a definite impact on science in the low countries. At the time the steam engine was introduced in the Netherlands (around 1850), an estimated number of 10,000 wind mills were in use. During the industrial revolution the use of coal as source of energy led to a gradual decline of the use of wind as a means to do work.

A renewed interest in wind energy was sparked due to the oil crisis of 1973 when Arab countries increased oil prices by $70 \%$ and reduced oil output every month. In the USA the precursor to the Department of Energy tasked NASA with research into utility-scale wind turbines. In cooperation with large companies like Boeing, Lockheed, and General Electric from the aerospace field, a series of prototype wind turbines were developed ranging from the 38 meter rotor diameter MOD-0 turbine of $100 \mathrm{~kW}$ rated power in 1975 , to the 97.5 meter rotor diameter MOD-5B turbine of $3.2 \mathrm{MW}$ rated power in 1987. It was soon discovered that the knowledge gained in the aerospace industry on aerodynamics and structures was not one to one transferable to the realm of the highly unsteady characteristics prevalent in wind turbine applications.

In 1955 the Reactor Centrum Nederland (RCN) was established in the Netherlands with the objective to secure the energy future of the country through the development of nuclear energy. This "atomic power" held the promise of vast quantities of inexpensive and clean energy. Gradually the awareness grew that radioactive nuclear waste would become a real problem and public opinion shifted away from nuclear energy. The oil crisis of 1973 resulted in 1976 in the foundation of the Energy research Centre of the Netherlands (ECN) that incorporated RCN as a daughter research institute.

ECN was tasked by the Dutch government with research into all possible renewable energy resources and provide the government with advice on energy policies. Unfortunately the political climate in the Netherlands was such that wind energy was politicized and regarded a left-wing hobby. More policies were directed towards increasing conventional power production from coal, natural gas, and oil, and towards increasing nuclear power. Moreover, wind energy was considered technology of the past, and certainly not an advancement in technology. The resolution of the oil crisis and a return to low cost oil from the Arab countries made the argument for investing in renewable energy very difficult. The result was an adverse business climate for wind turbine manufacturers in the Netherlands and a discouragement for the installation of wind turbines. 
At the present day some effort is put into increasing the share of wind energy to the total power production in the Netherlands by stimulating research and application of offshore wind farm power plants. This drive towards offshore locations is directly related to the highly populous western parts of the Netherlands that have the better wind climate. It should be noted that the current complaints of "horizon pollution" were also expressed several centuries ago when wind mills started to appear. Giving the local community their fair share of the proceeds could remove this impediment.

In 2015 the installed wind power capacity in the Netherlands was about $3.4 \mathrm{GW}$. The wind turbines contributed about $6.3 \%$ to the total electricity consumption in the Netherlands [4], which corresponds to about a $1.4 \%$ contribution of wind energy to the total energy consumption.

In the Kyoto Protocol [2] industrialized countries agreed to reduce their collective emissions of greenhouse gases in 2008-2012 by $5.2 \%$ compared to the year 1990. At the Paris climate conference in December 2015 [3] (COP21), 195 countries adopted a legally binding global climate deal to limit global warming to well below $2^{\circ} \mathrm{C}$. Another positive sign is that nowadays oil companies like Shell start to be active in off-shore wind farm construction and exploitation [92].

Despite these encouraging developments a few words on the current wave of "greenwashing" that proclaims that we can keep on doing the things we are currently doing, if only we start using "green" technologies like electric cars, photovoltaic solar panels and wind turbines. In light of mitigating the effects of carbon emissions and climate change, it should be noted that the installation of such technologies without removal of conventional fossil fuel based power generation will result in a net increase of greenhouse gasses to the detriment of all living plants and animals on the planet. For a substantial reduction of greenhouse gasses our current set of living arrangements will have to be reconsidered drastically [38] to overcome our addiction to vast amounts of energy.

\subsection{Wind Turbine Aerodynamics}

It was the quest for flying machines that drove the interest in the further development of a theory for aerodynamics around the start of the twentieth century. Some of the notable names that also greatly contributed to wind turbine and propeller aerodynamics are Joukowsky in Russia, Prandtl and Betz [11] in Germany, and Lancaster in England. It was Betz [10] in the introduction of a paper dedicated to wind energy who stated 


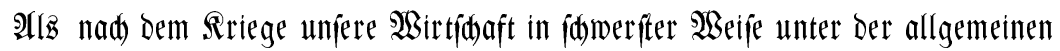
Rohlennot litt, lenfte fidt bie $\mathfrak{A}$ ufmerff́amfeit wieber ftart anberen Energiequeflen

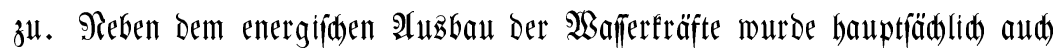
eine ftärtere heranzitehung Der 2 Sindenergie empfoblen.

The coal shortage mentioned by Betz in his recommendation for wind energy was due to the in kind reparations that Germany had to submit to after their defeat in WW-I. This bears some similarity with the current situation in which there is a growing hunger for energy while conventional resources are past their peak and climate change consequences are clear. The aerodynamic theories developed in that era by the research groups in Russia, Germany, and England are still in use today by wind turbine designers as blade-element-momentum (BEM) methods. An overview of the ideas behind BEM methods can be found in the reference work by de Vries [116] or the recent book by Sørensen [106], and a discussion of the work by Joukowsky can be found in [67] and [81]. Though strictly valid only for steady, incompressible, inviscid, quasi one-dimensional flows, the method is extended with various models that are tuned to experimental data and that try to compensate for the lack of physics in the underlying relations. The BEM method is still an area of active research at ECN in the Netherlands [99]. The justification of this approach is often found in the computational efficiency of the method and the resulting ability to perform hundreds of simulations for different flow cases in the design and certification phases of a new wind turbine.

After WW-II, the general availability of the digital computer in 1957 heralded the birth of computational fluid dynamics (CFD) without the need for classrooms full of human computers as shown in Figure 1.2 and described by Grier [44]. It was the start of research into areas that were unimaginable before and the beginning of an explosion of algorithms, theories, mathematical models, approximations, books, articles, and computer programs for the simulation the behavior of fluids under various circumstances.

One of the first methods that were implemented that could simulate the inviscid incompressible flow around three-dimensional geometries for incompressible and inviscid fluids was the so-called panel method by Hess and Smith [53] in 1962 working at the Douglas Aircraft Division. The advantage of the method was that the problem could be reduced to an integral equation on the surface of the geometry only; no volume discretization was necessary. This meant that flow problems for fairly complicated geometries could be numerically simulated. Though the initial implementation was for non-lifting bodies, the introduction of vortex elements soon after made it feasible to analyze lift carrying bodies too and numerically simulate flows 


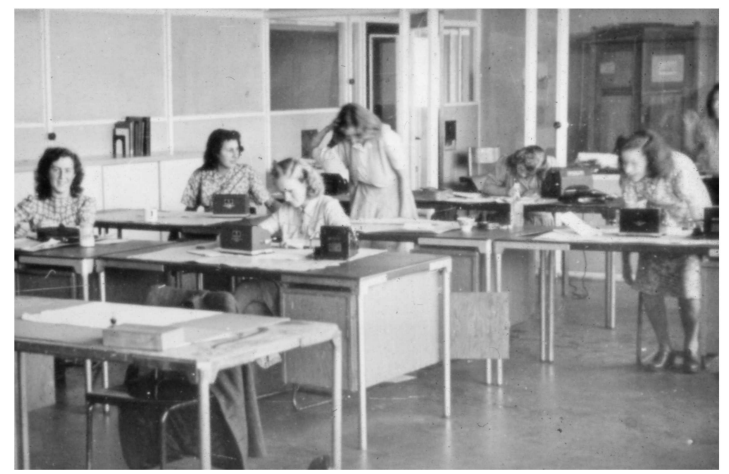

Figure 1.2: Human computers at NLR. Courtesy of H. Tijdeman.

around full aircraft configurations. In the Netherlands the development of such a panel method was carried out at NLR (Netherlands Aerospace Centre) by Labrujère and coworkers [68] in 1970. A logical next step that was undertaken was to use higher order approximations and increase the accuracy of the method [60],[57]. In the scientific community these panel methods are nowadays referred to as Boundary Element Methods, but due to a possible confusion with the blade-element-momentum methods that share the same acronym BEM, we will keep using the classical term "panel methods" in this thesis.

There is a very old relationship between wind energy and panel methods: George Green, the mathematician who discovered what was to be called Green's Theorem spent most of his days working in his windmill for grinding flour. His major work [40] forms the basis of the panel method and was selffinanced by the proceeds of wind energy. For a description of the history of the panel method and the various incarnations the reader is referred to references [25],[58], and [63].

The panel method was extended to the numerical simulation of linearized supersonic flows [24],[56], but due to the panel method's inability to simulate high speed transonic flows, the aerospace CFD community soon set it sights at the development of computational methods based on the steady Euler equations so that shock waves occurring in the flow around high speed airplanes could be simulated. Flow solvers that could simulate these flows around three-dimensional configurations in a production setting started to appear after 1980 .

The next step was to extend these solvers for the Euler equations for steady 
flow to viscous flows governed by the Reynolds-averaged Navier-Stokes (RaNS) equations. This necessitated new research in turbulence modeling and it was in the second half of the 1990's that such solvers were commonly available and could simulate flows with reasonable accuracy.

Later developments were directed towards unsteady flows, the interaction with deforming geometries, and the introduction of optimization strategies. Key words here are Large Eddy Simulation (LES), Fluid-Structure Interaction (FSI), higher order schemes, and adjoint equation methods. At the University of Twente several of these topic have been pursued in the framework of wind turbine aerodynamics in the last decade: Verhoeff [112] on an Euler method for rotating geometries, De Vries [114] on the simulation of synthetic jets with an unsteady RaNS method, and Jongsma [61] on an adjoint method for optimization of wind turbine blade designs.

Of course all these advances have only become feasible by the increasingly faster computers and continuous development of advanced algorithms like preconditioning and fast iterative solvers for systems of equations such as Krylov and multigrid methods. For the programming side of the CFD productivity rise, the advent of parallel and vector programming techniques are mentioned here. It is only recently that these unsteady RaNS or LES methods are applied for representative unsteady wind turbine flow conditions in combination with structural dynamics [50],[59].

At NLR another interesting development has been the so-called field panel method [91], [87] that was based on the full potential equation for isentropic flows. Its advantage was that only the part of the flow with non-negligible compressibility required a volume grid. The project, however, was put on hold due to the impractical quadratic computational costs. Later the project was reconsidered after the MLMI algorithm (see below) was found to reduce these cost substantially. Unfortunately, by that time the vast investments in numerical flow simulation methods based on the Euler equations, their successful application, and the prospect of extending the method to viscous flows was the reason the project was abandoned for good.

There was some continued development on panel methods in the 1980's for airplanes in arbitrary motion by Katz and Maskew [62] and on modeling the dynamic behavior of the wakes emanating from lifting wings by Hoeijmakers [57] at NLR in the Netherlands. The non-linearity due to the deforming and rolling-up vortex sheets as well as the quadratic computational character of the problem, however, prohibited widespread use.

For integral methods notable new algorithms were introduced in the late 1980's through the introduction of a geometric hierarchy of approximations which reduced the computational times for the evaluation of the integrals 
appearing in, for example, acoustics, electromagnetics, hydrodynamics, lubrification, magnetostatics, molecular dynamics, radiosity, and celestial mechanics. One of the earliest fast evaluation schemes is the Fast Multipole Method (FMM) introduced by Greengard [41] in 1987. In his thesis he used spherical harmonics expansions to approximate the inverse distance Green's function in the description of potential fields of particle systems in three dimensions. Hackbusch and Nowak in 1989 introduced a panel clustering scheme in which multipole expansions of clusters of nearby panels were combined. This was an extension of the use of multipole (Taylor series) expansions in the panel coordinates earlier by Hess and Smith [53] in 1962. Hess and Smith introduced the multipole expansion in order to avoid the computationally expensive evaluation of $\ln (x)$ and $\arctan (x)$ functions whenever possible. Instead of a series expansion Brandt and Lubrecht [23] in 1988 introduced interpolating polynomials in their Multi-Level MultiIntegration (MLMI) scheme. Interpolation was performed in both the panel and evaluation point coordinates in order to approximate the Green's function. This approach avoids the need for higher order derivatives of the Green's function and can be applied for general functions too. It has found widespread application in the field of contact mechanics and lubrication between deformable bodies [111]. The basic idea of the MLMI method was outlined already by Brandt [19] in 1981 and described briefly in [20] in 1987. The application of the MLMI method for a practical problem was demonstrated by Lubrecht and Ioannidis [72].

These multilevel algorithms were able to reduce the quadratic computational cost of the panel method substantially. However, at their time of discovery, the aerospace CFD community had mostly terminated the research into panel methods and was focused on RaNS flow solvers and turbulence modeling. Recent research on the development of panel methods for aerospace applications can be found in the publications by Moore, Peraire and Drela in [76], and Willis [119].

\subsection{Challenges}

The ongoing trend in wind turbine design is towards larger rotors, leading to increasing investment costs and related concerns regarding risk mitigation. An increase in size also leads to relatively flexible structures that are more susceptible to unsteady load occurrences. Fluctuating loading is inherent in unsteady wind turbine aerodynamics as for example by variations in wind speed and direction, irregular rotational speed of the blades, blade pitch actions, rotor yaw misalignment, blade deformations, and the dynamic 
character of the wake downstream of the rotor, to name a few. Wind turbine aerodynamics simulation tools that can predict the effects of unsteady flow on the pressure loading on the wind turbine rotor blades with sufficient accuracy are key to more economic wind turbine designs in the near future.

The challenge for wind turbine applications is to ultimately integrate a mix of simulation methods for unsteady aerodynamics, structural dynamics, and control strategies for use in a wind turbine engineering environment with balanced problem turnaround times and simulation accuracy. The success of flow solvers based on the Navier-Stokes equations, often using large computer systems (many cores), obscures the fact that for day-to-day practical use in a wind turbine engineering environment there is still a need for a method with higher fidelity than the blade-element-momentum methods currently in use in that mix of simulation methods while maintaining reasonable computational demands and short problem turnaround times on relatively small scale computer systems.

A possible solution to this challenge is the Viscous-Inviscid Interaction (VII) technology that was started in the 1970's when inviscid flow methods were coupled in strong interaction with a method solving the boundary layer equations in order to account for the effects of viscosity, specifically in the boundary layers. Figure 1.3 sketches such a decomposition of the flow domain into an outer region in which the flow is considered inviscid and an inner region where the effects of viscosity will be taken into account. An overview of the aspects involved in such an approach has been given by Lock and Williams [71]. As mentioned earlier, this research was almost completely abandoned once practically applicable RaNS based computational methods started to appear. Drela [31] was one of the first to implement a computational method that coupled a panel method for the inviscid external flow with an Integral Boundary Layer (IBL) method successfully for two-dimensional airfoils. His XFOIL computer program is nowadays open source, freely available [30], and the de-facto industry standard.

For wind turbine and helicopter rotor blades the XFOIL program was extended with a boundary layer approximation for the radial flow in the rotating airfoil boundary layer in a collaborative project of ECN, NLR, and Delft University of Technology by Snel, Houwink, Bosschers, and Van Rooij [103]. A similar approach that models a quasi-3D boundary layer flow, but now coupled with a fully 3D panel method, has been pursued by Ramos-García [93] in cooperation with Sørensen and Shen [94] in Denmark.

At NLR in the Netherlands, a successful implementation of a method solving the 3D full-potential equations in strong interaction with a finite difference method for the integral boundary layer equations found its way into 
Inviscid flow
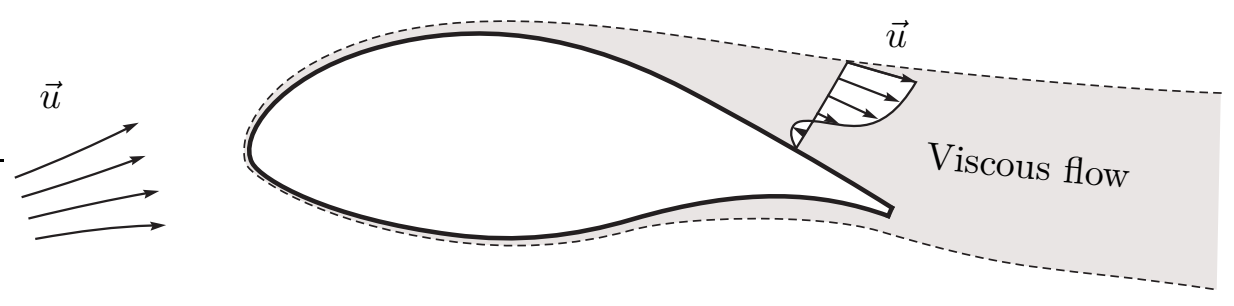

Figure 1.3: Domain decomposition into an inviscid flow region and a viscous flow region.

the MATRICS-V code by Van der Wees and Van Muijden [117]. Due to its ease of use and the fast problem turnaround times this computational method was part of the core aerodynamic design tools at Fokker Aircraft for the simulation of steady transonic flows about simple wing-body configurations.

With these successes in mind, a fully three dimensional and unsteady flow approach was initiated at ECN in 2004 by Van Garrel [36] that would be based on viscous-inviscid interaction technology; for the external flow a time-stepping low-order panel method was selected [37] and a strong coupling with a 3D integral boundary layer method would allow to account for the effects of viscosity even in the case of separated flows. This particular combination of models for the inviscid external flow and the viscous flow in the boundary layer keeps the mathematical problem restricted to the surface of the configuration for both models and thus avoids a timeconsuming volume discretization. The development of an unsteady 3D IBL method has been started by Özdemir [83],[84],[85],[86] and an investigation into a 3D viscous-inviscid interaction algorithm has been performed by $\mathrm{Bi}$ jleveld [12],[13] in collaboration with Veldman (see [107],[108],[109]) at the University of Groningen.

The development of a fully three-dimensional integral boundary layer method in strong interaction with a method for inviscid flows for the external flow is taking place at several other places, the most notable is the work by Drela [32].

For wind turbine applications the dynamic character of the deformation of the wake has to be taken into account in all unsteady flow situations. A substantial drawback, however, is the computational effort that grows quadratically with problem size $N$ when a straightforward panel method implementation for the rolling-up of the wake is used. To render such 
numerical simulations practical the current research therefore focuses on reducing the $\mathcal{O}\left(N^{2}\right)$ computer run times to $\mathcal{O}(N)$ through the development of a fast multilevel integral evaluation scheme capable of handling highly distorted wake geometries.

The current results offer the possibility to have a fresh look upon the mostly discontinued research of the 1970's and 1980's on panel methods, integral boundary layer methods, strong viscous-inviscid coupling, and fluid structure interaction. These technologies can become an important part of "medium fidelity" wind turbine simulation methods that bridge the gap between the low-fidelity BEM methods and the computationally expensive CFD methods. For engineering environments such balanced combination of higher accuracy and shorter simulation times is the ultimate goal.

\subsection{Thesis Outline}

In this thesis we focus on the development of the theory and the practical implementation of a fast multilevel integral transform. We use this multilevel scheme in a low-order panel method and demonstrate that for the simulation of the wake flow of wind turbine rotors the computational burden is reduced to $\mathcal{O}(N)$, that is, the computational effort grows linearly with problem size $N$. This thesis argues for this case as follows:

- We build the governing equations and state the assumptions under which they are valid in Chapter 2. The continuous mathematical description of the resulting boundary integral equations is given and the boundary conditions required to solve the equations. We describe the equations governing the evolution of the rotor wake in time and give the expressions for the pressure distribution over the surface of the rotor blades and the forces and moments acting locally on the surface.

- We describe the discretization of the mathematical model in the form of a low-order panel method (Chapter 3) and explain how the time-dependent blade motion is handled. For the implemented panel method we perform several verification tests. We compute the solid angle at points in the interior of a body and compare the results with the exact solution. For a tri-axial ellipsoid in uniform onset flow and a rotating ellipsoid in still air exact solutions are available. For these two cases the accuracy of the panel method is shown to be $\mathcal{O}\left(h^{2}\right)$ in the potential function, with $h$ a characteristic discretization length. A wing with elliptic planform is constructed and equipped 
with von Kármán-Trefftz airfoil cross-sections for which exact solutions are available for two-dimensional flows. The panel method accuracy in the pressure distribution is shown to be somewhat less than $\mathcal{O}(h)$.

- We develop a fast multilevel integral transform method that is capable of handling highly irregular wake sheets as occurring in the simulation of wind turbine wake flows with a panel method (Chapter 4). We start the explanation on the basis of a one-dimensional integral transform involving a smooth scalar function integrand and a point discretization. We extend the method to asymptotically smooth kernels with localized singularities and describe the algorithm for higher dimensions. We discuss higher order surface singularity distributions as well as more general kernel (integrand) functions.

The implemented multilevel method (designated MLMIC) is submitted to verification tests. We first show, for a test case representing the growth of a wind turbine wake in time, that the computational effort of the method is $\mathcal{O}(N)$ and that the error level can be controlled. We repeat the solid angle test case for the panel method and confirm that the accuracy of the solution can be controlled by two free parameters: the order of the interpolating function and the box size at the finest grid level.

- In Chapter 5 we show the results for the combined panel method and multilevel scheme for a practical wind turbine application. The MEXICO wind tunnel experiment is numerically simulated for three wind turbine operating conditions and we compare the pressure distributions at five rotor blade cross sections with the experimental data and with the results of a state-of-the-art numerical method based on the Reynolds-averaged Navier-Stokes equations. The evaluation of the fluid velocities in the rotor wake with the new MLMIC method is shown to be a factor 150 faster than with a conventional panel method.

- We give a summary of the work in Chapter 6, present the main conclusions, and give an outlook on possible future research directions. The current work repositions panel methods in the computational landscape as valuable medium fidelity computational design method for wind turbine engineering. 


\section{Potential Flow Model}

\subsection{Introduction}

We consider the mathematical model for three-dimensional unsteady flow around wind turbine rotors and start the discussion with the Helmholtz Decomposition. This theorem (see Appendix C) states that a continuously differentiable velocity vector field $\vec{u}(\vec{x})$ around an arbitrary body in 3D space can be described equivalently in terms of source $\sigma_{v}(\vec{x})$ and vorticity $\vec{\omega}_{v}(\vec{x})$ distributions throughout the volume plus an irrotational and solenoidal onset flow $\vec{u}_{\infty}(\vec{x})$, as depicted in Figure 2.1. The two descriptions can be converted into one another through the equations indicated in the same figure. A common phrase is that the velocity field is "induced" by the source and vorticity distributions. Mathematically the two descriptions are equivalent and no cause and effect relationship exist between the two flow field representations, although the word "induced" would suggest otherwise.

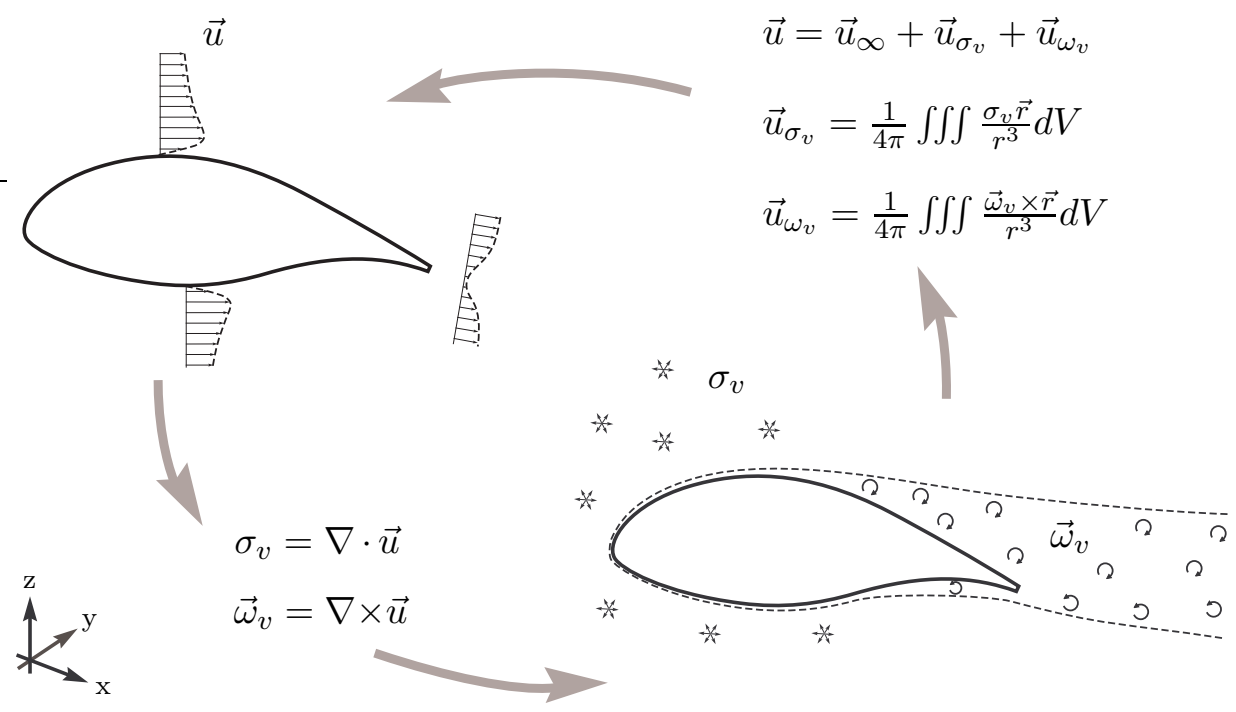

Figure 2.1: Equivalent flow field representations in terms of velocity vector field $\vec{u}(\vec{x})$ or in terms of source $\sigma_{v}(\vec{x})$ and vorticity $\vec{\omega}_{v}(\vec{x})$ volume distributions.

Helmholtz' theorem suggests a split of the domain into a region near the configuration where $\nabla \times \vec{u} \neq \overrightarrow{0}$ and the effects of viscosity are significant 
and an external inviscid flow region. In the present study incompressible flow is assumed, that is, $\nabla \cdot \vec{u}=0$.

We will model the external inviscid flow field and restrict the volumetric source and vorticity distributions to the body and wake surfaces as indicated in Figure 2.2. This leads to a divergence free and irrotational flow everywhere except across these surfaces. The problem is thus reduced to integral equations, involving the boundary of the configuration only, that describe the flow anywhere in the field. Under certain conditions (see Appendix D) this approach can be justified in the case of wind shear in the onset flow field of a wind turbine, that is, when the onset flow field has a rotational component.

It will be possible to account for the effects of viscosity through the concept of a "transpiration velocity" normal to the surface as introduced by Lighthill [70]. The boundary layer displacement thickness necessary to determine this normal velocity can be obtained from a method for solving the boundary layer equations. A strong viscous-inviscid interaction scheme (see Lock and Williams [71], Veldman [108]) would make it possible to account for mildly separated boundary layer flows.

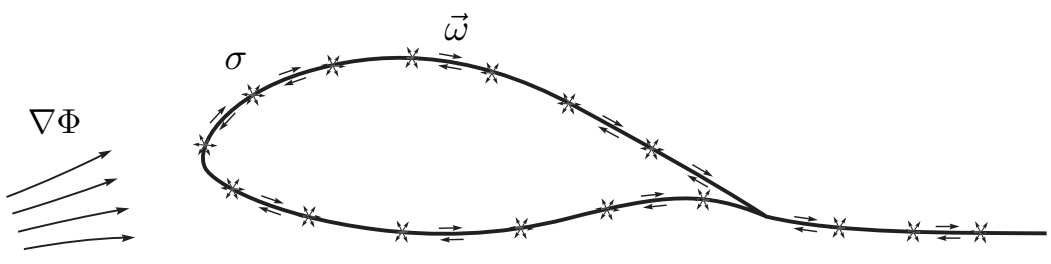

Figure 2.2: Flow field approximation.

\subsection{Governing Equations}

The Navier-Stokes equations describe general fluid flows and express the physical principles of conservation of mass, momentum, and energy. For a point $\vec{x}$ in volume $V \in \mathbb{R}^{3}$ at time $t$, the equations for conservation of mass, momentum, and energy in differential form are

$$
\begin{gathered}
\frac{\partial \rho}{\partial t}+\nabla \cdot(\rho \vec{u})=0, \\
\frac{\partial(\rho \vec{u})}{\partial t}+\nabla \cdot(\rho \vec{u} \vec{u})+\nabla p-\rho \vec{g}-\nabla \cdot \overline{\bar{\tau}}=\overrightarrow{0}, \quad \text { and } \\
\frac{\partial(\rho E)}{\partial t}+\nabla \cdot(\rho E \vec{u})+\nabla \cdot(p \vec{u})-\rho \vec{g} \cdot \vec{u}-\dot{Q}+\nabla \cdot \vec{q}-\nabla \cdot(\overline{\bar{\tau}} \cdot \vec{u})=0,
\end{gathered}
$$


respectively, with $\rho(\vec{x}, t)$ the mass density, $\vec{u}(\vec{x}, t)$ the fluid velocity, pressure $p(\vec{x}, t), \vec{g}$ the gravitational acceleration vector, and $\overline{\bar{\tau}}(\vec{x}, t)$ the viscous stress tensor. The total energy per unit mass is denoted by $E(\vec{x}, t)$ and the term $\dot{Q}(\vec{x}, t)$ in the energy equation $(2.3)$ is the rate of volumetric heating that works on the fluid volume directly, for example by radiation, and has dimension $\left[\mathrm{J} \cdot \mathrm{m}^{-3} \cdot \mathrm{s}^{-1}\right]$. Heat transfer due to thermal conduction is accounted for by the term with heat flux vector $\vec{q}(\vec{x}, t)$ that has dimension $\left[\mathrm{J} \cdot \mathrm{m}^{-2} \cdot \mathrm{s}^{-1}\right]$.

To close the system of equations, the Navier-Stokes equations are supplemented by two equations of state and two constitutive relations. The latter model the viscous stress tensor $\overline{\bar{\tau}}(\vec{x}, t)$ and the heat flux vector $\vec{q}(\vec{x}, t)$ in terms of available variables. See Appendix B for more details.

We consider the fluid dynamics equations for wind turbine applications where in normal operation the local flow velocities occurring at the rotor blades are at most $30 \%$ of the speed of sound, that is, Ma $\leq 0.3$. Away from the wind turbine rotor the local Mach number is even lower. The flow is therefore assumed to be incompressible and the effects of heating are considered negligible. Fluid mass density is considered constant. The effects of viscosity are assumed to be negligible due to the high operational Reynolds numbers. These considerations make it feasible to reduce the set of equations. For unsteady incompressible flow, the equation for the conservation of mass reduces to

$$
\nabla \cdot \vec{u}=0 .
$$

Although the equation does not have an explicit time derivative term, unsteady boundary conditions will introduce time dependence in the solution. The equations expressing conservation of momentum for unsteady, incompressible, inviscid flows read

$$
\rho_{\infty} \frac{\partial \vec{u}}{\partial t}+\rho_{\infty}(\vec{u} \cdot \nabla) \vec{u}+\nabla\left(p-\rho_{\infty} \vec{g} \cdot \vec{x}\right)=\overrightarrow{0} .
$$

A significant reduction in complexity can be achieved when it is assumed that rotational flow is confined to infinitesimal thin boundary layer and wake regions, and is irrotational everywhere else, that is $\nabla \times \vec{u}=\overrightarrow{0}$. This allows us to write the velocity vector field $\vec{u}(\vec{x}, t)$ as the gradient of a scalar velocity potential function $\Phi(\vec{x}, t)$ :

$$
\vec{u}=\nabla \Phi .
$$

Substitution of equation (2.6) in the continuity equation (2.4) gives the Laplace equation for the velocity potential in domain $V$ :

$$
\nabla \cdot \nabla \Phi=0 .
$$


Substituting equation (2.6) in the equations for conservation of momentum (2.5) results in the Bernoulli equation for unsteady potential flow

$$
\frac{\partial \Phi}{\partial t}+\frac{1}{2} \nabla \Phi \cdot \nabla \Phi+\frac{p}{\rho_{\infty}}-\vec{g} \cdot \vec{x}=C(t)
$$

in the whole domain as derived in Appendix B.2

\subsection{Boundary Integral Equation}

It is assumed that domain $V$ can be decomposed into a set of non-overlapping volumes $V_{m}$ with boundaries $\partial V_{m}$ as depicted in Figure 2.3. Let $S_{m, k}$ be the part of the boundary that the two volumes $V_{m}$ and $V_{k}$ have in common:

$$
S_{m, k}=\partial V_{m} \cap \partial V_{k}, \quad m \neq k .
$$

When approaching $S_{m, k}$ from volume $V_{m}$ that side of the surface is indicated by $S_{m}$. and likewise $S_{. k}$ for the other side. The surface $S$ of the configuration and its wake is now described by the complete set of boundaries:

$$
S=\cup S_{m, k} .
$$

The velocity potential function in the Laplace equation (2.7) can be expressed (see Appendix E) for point $\vec{x}$ in volume $V$ in terms of a reference onset velocity potential $\Phi_{\infty}(\vec{x}, t)$ and perturbation velocity potential contributions $\varphi_{\mu}(\vec{x}, t)$ and $\varphi_{\sigma}(\vec{x}, t)$ from integrals involving dipole singularity distributions $\mu(\vec{y}, t)$ and source singularity distributions $\sigma(\vec{y}, t)$, respectively, on the boundaries $S_{m, k}$, that is

$$
\Phi=\Phi_{\infty}+\varphi_{\mu}+\varphi_{\sigma},
$$

The perturbation velocity potentials induced at point $\vec{x}$ by the dipole and source distributions on all surfaces $S_{m, k}$ are defined by

$$
\begin{aligned}
\varphi_{\mu}(\vec{x}, t) & =\frac{-1}{4 \pi} \iint_{S} \mu \frac{\bar{n}_{m} \cdot \vec{r}}{r^{3}} d S, \\
\varphi_{\sigma}(\vec{x}, t) & =\frac{-1}{4 \pi} \iint_{S} \sigma \frac{1}{r} d S,
\end{aligned}
$$

where $\bar{n}_{m}(\vec{y}, t)$ is the unit normal vector in $\vec{y} \in S_{m, k}$ that is pointing into volume $V_{m}$. The vector $\vec{r}$ is defined as the vector from a point $\vec{y}$ on the 


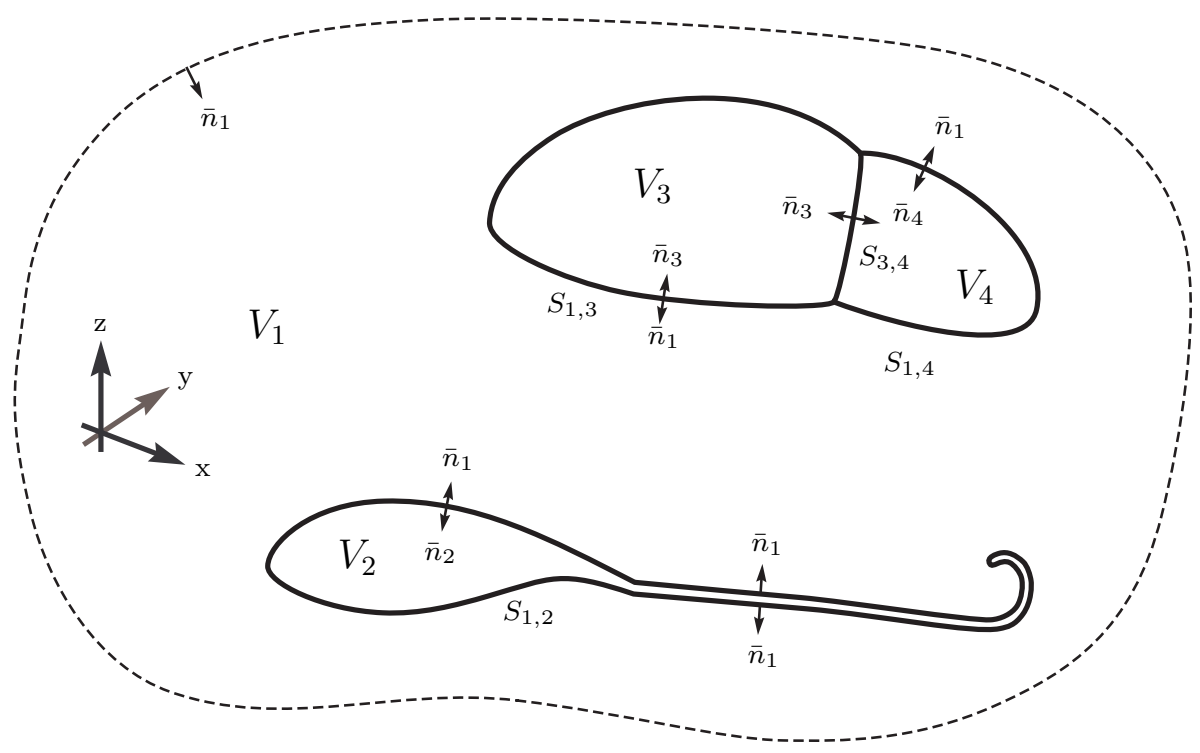

Figure 2.3: Flow domain $V \in \mathbb{R}^{3}$ is the union of non-overlapping volumes $V$ and inner boundaries $S_{m, k}$ that separate volume $V_{m}$ from volume $V_{k}$. Unit normal vector $\bar{n}_{m}$ is defined to point into volume $V_{m}$.

surface to evaluation point $\vec{x}$, whereas the distance between the two points is denoted by $r$, that is,

$$
\vec{r}=\vec{x}-\vec{y}, \quad r=|\vec{r}|, \quad \text { for } \quad \vec{y} \in S_{m, k} .
$$

For problems where the evaluation point $\vec{x}$ and the boundary $S_{m, k}$ are moving relative to each other, we have $\vec{r}=\vec{r}(t)$.

The dipole strength $\mu(\vec{y}, t)$ and the source strength $\sigma(\vec{y}, t)$ at point $\vec{y}$ at the surface, are related to the velocity potential values $\Phi_{m}(\vec{y}, t)$ and $\Phi_{k}(\vec{y}, t)$ on both sides of the surface by

$$
\begin{aligned}
\mu(\vec{y}, t) & =-\left(\Phi_{m}-\Phi_{k}\right), \\
\sigma(\vec{y}, t) & =\nabla\left(\Phi_{m}-\Phi_{k}\right) \cdot \bar{n}_{m} .
\end{aligned}
$$

As shown in Appendix E, the integral for the dipole singularity distribution causes a jump at point $\vec{y} \in S_{m, k}$ in the velocity potential of strength $\mu(\vec{y}, t)$ across the surface:

$$
\lim _{\varepsilon \rightarrow 0} \varphi\left(\vec{y} \pm \varepsilon \bar{n}_{m}, t\right)=\varphi_{\sigma}^{p}(\vec{y}, t)+\varphi_{\mu}^{p}(\vec{y}, t) \mp \frac{1}{2} \mu(\vec{y}, t) .
$$

The two $\varphi^{p}$ terms on the right-hand side of equation (2.15) are to be interpreted as Principal Value or Finite Part integrals over the complete set 
of inner surfaces, with an infinitesimal region around the singular point excluded from the surface of integration.

\subsection{Boundary Conditions}

\subsubsection{Introduction}

Appropriate boundary conditions are required to find a solution for the Laplace equation (2.7) for the potential field $\Phi(\vec{x}, t)$ as formulated in terms of reference potential field $\Phi_{\infty}(\vec{x}, t)$ plus source and dipole perturbation potential fields $\varphi_{\sigma}(\vec{x}, t)$ and $\varphi_{\mu}(\vec{x}, t)$.

For surfaces of thick bodies we will use internal Dirichlet boundary conditions as introduced by Morino and Kuo [77] and later popularized by Maskew [73]. This formulation assumes that we are only interested in the flow field on one side of the surface, and that the flow inside the volume at the other side of the surface is of no interest and can be prescribed. This excludes the use of internal Dirichlet boundary conditions for the flow over e.g. infinitesimal thin surfaces.

\subsubsection{Body Surface}

For thick bodies, the internal Dirichlet formulation will be used assuming that only the solution in the volume on one side of the surface is of interest. Let $S_{m}$. denote the side of surface $S_{m, k}$ in volume $V_{m}$ where we want to obtain a solution of the physical flow problem, and let $S_{. k}$ denote the side of the surface $S_{m, k}$ in volume $V_{k}$ that is considered non-physical and contains a fictitious flow.

The boundary condition at surface side $S_{m}$. is such that the flow velocity at the surface in normal direction, is equal to normal component of the surface velocity $\vec{u}_{S}(\vec{x}, t)$, plus a specified outflow velocity $v_{n}(\vec{x}, t)$ in normal direction relative to the moving boundary:

$$
\nabla \Phi_{m} \cdot \bar{n}_{m}=\vec{u}_{S} \cdot \bar{n}_{m}+v_{n}, \quad \vec{x} \rightarrow S_{m} .
$$

The distribution of the normal velocity $v_{n}$ can be used for example to simulate the boundary layer displacement thickness effects or for the simulation of inflow through a suction slot. The local surface velocity $\vec{u}_{S}$ may be composed of solid body rotation, surface translation, surface rate of deformation and so on.

Suppose the velocity potential in the fictitious flow domains $V_{k}$ is known in 
advance

$$
\Phi_{k}=\Phi_{k}(\vec{x}, t), \quad \vec{x} \in V_{k},
$$

and let

$$
\vec{u}_{k}(\vec{x}, t)=\nabla \Phi_{k}, \quad \vec{x} \in V_{k} .
$$

For point $\vec{x} \in V_{k}$ the boundary integral equation (2.9) reads

$$
\varphi_{\mu}(\vec{x}, t)+\varphi_{\sigma}(\vec{x}, t)=\Phi_{k}(\vec{x}, t)-\Phi_{\infty}(\vec{x}, t), \quad \vec{x} \in V_{k} .
$$

In the limit of point $\vec{x}$ approaching the surface $S_{. k}$ at point $\vec{y}$, equation (2.9) is expressed in terms of Principal Value and Finite Part integrals as

$$
\frac{1}{2} \mu+\varphi_{\mu}^{p}(\vec{x}, t)+\varphi_{\sigma}^{p}(\vec{x}, t)=\Phi_{k}(\vec{x}, t)-\Phi_{\infty}(\vec{x}, t), \quad \vec{x} \rightarrow \vec{y} \in S_{. k} .
$$

Substitution of the boundary condition (2.16) at $S_{m}$. and the known velocity potential in volume $V_{k}(2.17)$ in the definition of the source strength (2.14), gives an expression for the source strength in terms of known quantities:

$$
\sigma(\vec{y}, t)=\left(\vec{u}_{S}-\vec{u}_{k}\right) \cdot \bar{n}_{m}+v_{n}, \quad \vec{y} \in S_{m, k} .
$$

The boundary integral equation (2.19) at $\vec{x} \rightarrow S_{. k}$ now gives an expression involving the unknown dipole strength $\mu(\vec{y}, t)$ as a function of known quantities. The surface gradient of the dipole strength (2.13) gives us at the surface side $S_{m}$. of interest for the tangential component of the velocity

$$
\nabla_{S} \Phi_{m}(\vec{x}, t)=\nabla_{S} \Phi_{k}-\nabla_{S} \mu, \quad \vec{x} \rightarrow S_{m} .
$$

The surface gradient of the velocity potential $\Phi_{k}$ is the velocity vector component of $\vec{u}_{k}$ tangential to the surface and can be written as

$$
\nabla_{S} \Phi_{k}=\bar{n}_{m} \times\left(\vec{u}_{k} \times \bar{n}_{m}\right) .
$$

Combining the normal velocity from boundary condition (2.16), the expression for the source strength (2.21), and the tangential velocity (2.22) gives an expression for the velocity at the surface in the inertial coordinate system:

$$
\nabla \Phi_{m}(\vec{x}, t)=\left(\vec{u}_{S} \cdot \bar{n}_{m}+v_{n}\right) \bar{n}_{m}+\nabla_{S} \Phi_{k}-\nabla_{S} \mu, \quad \vec{x} \rightarrow S_{m} .,
$$

which can also be written as

$$
\vec{u}_{m}(\vec{x}, t)=\vec{u}_{k}+\sigma \bar{n}_{m}-\nabla_{S} \mu, \quad \vec{x} \rightarrow S_{m} .
$$

Equation (2.25) states that the velocity at the surface side of interest is composed of a known base flow field $\vec{u}_{k}$ and a perturbation flow field due to 
the source and dipole singularity distributions. Substitution of the expression for the source strength (2.21) in this equation gives another expression for the velocity in the inertial coordinate system at point $\vec{x} \rightarrow S_{m}$. :

$$
\vec{u}_{m}=\vec{u}_{k}-\left(\vec{u}_{k} \cdot \bar{n}_{m}\right) \bar{n}_{m}+\left(\vec{u}_{S} \cdot \bar{n}_{m}\right) \bar{n}_{m}+v_{n} \bar{n}_{m}-\nabla_{S} \mu .
$$

Although mathematically equations (2.25) and (2.26) are equivalent, for the computation of the velocity at the nodes of the grid on the surface, equation (2.26) is preferred as it avoids a possible interpolation of the source strength $\sigma(\vec{y}, t)$ defined in (2.21). In equation (2.26) only the contribution of the specified outflow velocity $v_{n}$ possibly needs to be interpolated, assuming that the surface velocity $\vec{u}_{S}$ and the fictitious velocity $\vec{u}_{k}$ are quantities known at any location on the surface.

For an observer at point $\vec{x} \in S_{m}$., moving with the local surface velocity $\vec{u}_{S}$ the relative velocity that is experienced will be

$$
\vec{u}_{\text {rel }}(\vec{x}, t)=\vec{u}_{m}-\vec{u}_{S}
$$

Combined with (2.26) this expression becomes

$$
\vec{u}_{\text {rel }}=\vec{u}_{k}-\left(\vec{u}_{k} \cdot \bar{n}_{m}\right) \bar{n}_{m}-\vec{u}_{S}+\left(\vec{u}_{S} \cdot \bar{n}_{m}\right) \bar{n}_{m}+v_{n} \bar{n}_{m}-\nabla_{S} \mu .
$$

The velocity relative to the surface is composed of the tangential components of the fictitious velocity, the surface velocity, and the dipole gradient, plus a contribution in normal direction from the outflow velocity $v_{n}$. The expression for the relative velocity can also be written as

$$
\vec{u}_{\text {rel }}=\bar{n}_{m} \times\left(\left(\vec{u}_{k}-\vec{u}_{S}\right) \times \bar{n}_{m}\right)+v_{n} \bar{n}_{m}-\nabla_{S} \mu .
$$

Still, the velocity potential field $\Phi_{k}$, and consequently velocity field $\vec{u}_{k}$, has to be defined in the fictitious domains. This gives some freedom to base this choice on the properties that the resulting set of equations for the solution in the physical domain will have. A choice that is expected to give small numerical errors is one that results in a smooth and low-gradient source and dipole distributions.

Here it is decided to follow Morino and Kuo [77] and Maskew [73] and set the fictitious flow field equal to the onset flow field, that is, $\Phi_{k}=\Phi_{\infty}$, and $\vec{u}_{k}=\vec{u}_{\infty}$. The definition of the dipole strength (2.13) gives $\varphi_{m}=-\mu$ for the perturbation potential at the exterior side of the surface. Assuming a known surface velocity $\vec{u}_{S}$ and known outflow velocity in normal direction $v_{n}$, the following set of equations determines the velocity distribution at the surface: 
$\Phi_{k}=\Phi_{\infty}, \vec{u}_{k}=\vec{u}_{\infty}:$

$$
\begin{aligned}
\sigma & =\left(\vec{u}_{S}-\vec{u}_{\infty}\right) \cdot \bar{n}+v_{n}, & & \\
\frac{1}{2} \mu+\varphi_{\mu}^{p} & =-\varphi_{\sigma}^{p}, & & \vec{x} \rightarrow S_{\cdot k}, \\
\vec{u}_{m} & =\vec{u}_{\infty}+\sigma \bar{n}-\nabla_{S} \mu, & & \vec{x} \rightarrow S_{m} .
\end{aligned}
$$

Alternative choices for the fictitious velocity potential field $\Phi_{k}$ and velocity field $\vec{u}_{k}$ are also possible:

$\Phi_{k}=0, \vec{u}_{k}=\overrightarrow{0}:$

In this option the fictitious flow field is set equal to zero. Assuming a known surface velocity $\vec{u}_{S}$ and known normal outflow velocity $v_{n}$, this gives the following set of equations to determine the velocity distribution at the surface:

$$
\begin{aligned}
\sigma & =\vec{u}_{S} \cdot \bar{n}+v_{n}, & & \\
\frac{1}{2} \mu+\varphi_{\mu}^{p} & =-\varphi_{\sigma}^{p}-\Phi_{\infty}, & & \vec{x} \rightarrow S_{. k}, \\
\vec{u}_{m} & =\sigma \bar{n}-\nabla_{S} \mu, & & \vec{x} \rightarrow S_{m} .
\end{aligned}
$$

Note that for a stationary surface and zero normal outflow velocity the source distribution is not required, i.e. $\sigma=0$, and that the dipole strength is now proportional to the total velocity at the surface.

$\Phi_{k}=\Phi_{\infty}, \vec{u}_{k}=\vec{u}_{\infty}, \Phi_{m}=0:$

For separated flows, as for example occurring behind blunt bodies, an option has been introduced by Carmichael and Erickson [24] to set the internal potential equal to the onset potential and set the external potential to zero. This fixes the jump in potential across the surface and results in a specified strength of the dipole distribution.

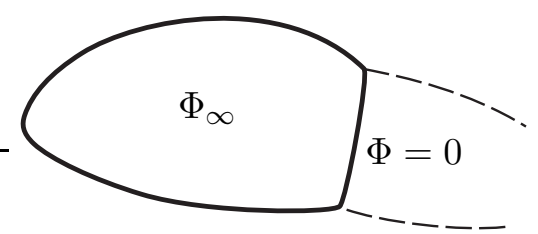

Figure 2.4: Base flow boundary conditions.

Assuming a known surface velocity $\vec{u}_{S}$, the following set of equations results to determine the velocity distribution at that part of the sur- 
face:

$$
\begin{array}{rlrl}
\mu & =\Phi_{\infty}, & \\
\varphi_{\sigma}^{p}=-\varphi_{\mu}^{p}-\frac{1}{2} \mu, & & \vec{x} \rightarrow S_{. k}, \\
\vec{u}_{m}=\left(\vec{u}_{\infty} \cdot \bar{n}+\sigma\right) \bar{n}, & & \vec{x} \rightarrow S_{m} . \\
v_{n}=\sigma-\left(\vec{u}_{S}-\vec{u}_{\infty}\right) \cdot \bar{n} . &
\end{array}
$$

Without further actions a discontinuity in the potential occurs at the edges of the base geometry. The corresponding discrete vortices can be counteracted by introducing wake surfaces emanating from these edges, as is indicated in Figure 2.4 by the dashed lines. The source strength can be split into an unknown part and a known part $\sigma=\sigma_{u}+\sigma_{k}$ which gives the possibility to rewrite above equations as

$$
\begin{array}{rlrl}
\mu & =\Phi_{\infty}, & \\
\sigma_{k} & =\left(\vec{u}_{S}-\vec{u}_{\infty}\right) \cdot \bar{n}, & \\
\varphi_{\sigma_{u}}^{p} & =-\varphi_{\mu}^{p}-\frac{1}{2} \mu-\varphi_{\sigma_{k}}^{p}, & & \vec{x} \rightarrow S . k \\
\vec{u}_{m} & =\left(\vec{u}_{\infty} \cdot \bar{n}+\sigma\right) \bar{n}, & & \vec{x} \rightarrow S_{m} . \\
v_{n} & =\sigma_{u} . &
\end{array}
$$

\subsubsection{Wake Surface}

At the trailing edge of a lifting body, the point where the flow leaves the surface (see Figures 2.1 and 2.2) wake surfaces are explicitly added in the potential flow model. A Kutta condition will be imposed at the trailing edge so that a smooth flow with finite velocity at that point results. Here, the linear Kutta condition that was introduced by Morino and Kuo [77] is used. The condition equates the dipole strength at the first point of the wake to the jump in dipole strengths across the trailing edge:

$$
\mu_{w_{t e}}=\llbracket \varphi \rrbracket_{t e}=\llbracket \mu \rrbracket_{t e} .
$$

For the evolution of the wake we will make use of the theorems of Helmholtz and Kelvin for vorticity dynamics (see Batchelor [6], Cottet and Koumoutsakos [26], Saffman [97]) that state that in inviscid incompressible flow the vorticity field moves with the fluid and its strength remains constant.

In incompressible inviscid flows subjected to a conservative force field the evolution of the vorticity field can be obtained by applying the curl operator to the momentum conservation equation (2.2). After some manipulation, the resulting Lagrangian description is

$$
\frac{\mathrm{D} \vec{\omega}}{\mathrm{D} t}=\vec{\omega} \cdot \nabla \vec{u} .
$$




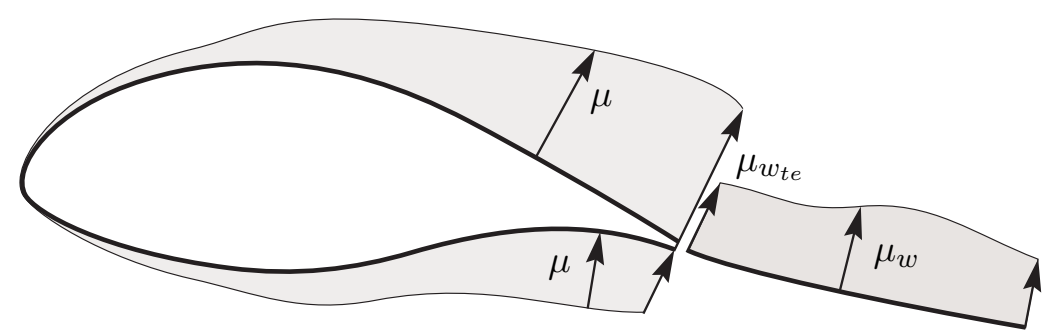

Figure 2.5: Trailing edge Kutta condition.

An identical relationship is valid for material line elements $\delta \vec{l}$ where $\vec{\omega}$ in above equation is replaced by $\delta \vec{l}$. We can thus conclude that in these flows, vortex lines behave as material line elements. Kelvin's circulation theorem for incompressible inviscid flows reads

$$
\frac{\mathrm{D} \Gamma}{\mathrm{D} t}=0
$$

where circulation $\Gamma$ is defined by a surface integral of the flux of vorticity through a cross-section of a vortex tube, or recast into a contour integral around the vortex tube with the help of Stokes' theorem (see Appendix A)

$$
\Gamma=\iint_{S} \vec{\omega} \cdot \bar{n} d S=\int_{\partial S} \vec{u} \cdot \bar{\tau} d s
$$

with $\bar{n}$ the unit normal vector to the cross-section at surface $S$, and $\bar{\tau}$ the unit vector tangential to the contour $\partial S$.

From above equations it can be concluded that in incompressible inviscid flows a tube of vorticity, though in general stretching and deforming, preserves its identity when moving with the velocity field. In terms of the evolution of wake element position $\vec{x}_{w}$ and wake element dipole strength $\mu_{w}$ the corresponding equations are

$$
\frac{\mathrm{d} \vec{x}_{w}}{\mathrm{~d} t}=\vec{u}, \quad \vec{x}_{w}\left(t_{0}\right)=\vec{x}_{t e}\left(t_{0}\right),
$$

and the material derivative of the wake dipole strength

$$
\frac{\mathrm{D} \mu_{w}}{\mathrm{D} t}=0, \quad \mu_{w}\left(t_{0}\right)=\mu_{w_{t e}}\left(t_{0}\right),
$$

where $t_{0}$ is the time of wake element creation. 
Differentiation of the potential field contributions (2.9) with respect to $\vec{x}$ gives an expression for the velocity field:

$$
\vec{u}(\vec{x}, t)=\vec{u}_{\infty}+\vec{u}_{\mu}+\vec{u}_{\sigma},
$$

where the perturbation velocities induced at point $\vec{x}$ by the dipole and source distributions on surface $S_{m, k}$ can be shown to be

$$
\begin{aligned}
& \vec{u}_{\mu}(\vec{x}, t)=\frac{-1}{4 \pi} \iint_{S}\left(\bar{n}_{m} \times \nabla \mu\right) \times \frac{\vec{r}}{r^{3}} d S+\frac{-1}{4 \pi} \int_{\partial S} \mu \frac{\vec{r}}{r^{3}} \times d \vec{l}, \\
& \vec{u}_{\sigma}(\vec{x}, t)=\frac{1}{4 \pi} \iint_{S} \sigma \frac{\vec{r}}{r^{3}} d S .
\end{aligned}
$$

The velocity field associated with a dipole distribution is equivalent to the induced velocity by a surface vorticity distribution $\vec{\gamma}$ of strength $\vec{\gamma}=-\bar{n} \times$ $\nabla \mu$ plus the velocity induced by a discrete vortex filament $\Gamma$ of strength $\Gamma=\mu$ along the edge of $S$ (see Hess [52], Hoeijmakers [58],[57]). Though the line integral in (2.38) is along the contour of the surface, in general such a contribution appears whenever there is a jump in the dipole distribution.

The advection of the wake is performed by integrating (2.35) over a time interval. In this integration the local velocity $\vec{u}$ is required at wake element positions $\vec{x}_{w}$. For each point an evaluation of the integrals in Equations (2.38) and (2.39) over the surface and along its edge is needed.

\subsection{Pressure, Forces, and Moments}

An expression for the pressure $p$ can be obtained from the unsteady Bernoulli equation (2.8) by relating upstream flow quantities with perturbed local quantities. Let $p_{\infty}$ be the undisturbed upstream pressure, and let $\Phi_{\infty}(\vec{x}, t)$ be the upstream total velocity potential, so that the unperturbed onset velocity is $\vec{u}_{\infty}=\nabla \Phi_{\infty}$. Likewise the perturbed local quantities are pressure $p$, total velocity potential $\Phi=\Phi_{\infty}+\varphi_{m}$, and local flow velocity $\vec{u}_{m}=\nabla \Phi_{\infty}+\nabla \varphi_{m}$. Substituted in the Bernoulli equation (2.8) this results in

$$
\frac{p-p_{\infty}}{\frac{1}{2} \rho_{\infty}}=\vec{u}_{\infty} \cdot \vec{u}_{\infty}-\vec{u}_{m} \cdot \vec{u}_{m}-2 \frac{\partial \varphi_{m}}{\partial t}+2 \vec{g} \cdot\left(\vec{x}-\vec{x}_{\mathrm{ref}}\right),
$$

where $\vec{x}_{\text {ref }}$ is defined as the point where the pressure $p$ is equal to the undisturbed pressure upstream $p_{\infty}$ when the fluid is at rest.

For the definition of a pressure coefficient we first introduce the material derivative of the perturbation potential $\varphi_{m}$ at a point on the surface moving 
with velocity $\vec{u}_{S}$ :

$$
\begin{aligned}
\frac{\mathrm{D}_{S} \varphi_{m}}{\mathrm{D} t} & =\frac{\partial \varphi_{m}}{\partial t}+\vec{u}_{S} \cdot \nabla \varphi_{m} \\
& =\frac{\partial \varphi_{m}}{\partial t}+\vec{u}_{S} \cdot\left(\vec{u}_{m}-\vec{u}_{\infty}\right) .
\end{aligned}
$$

We define the reference velocity $\vec{v}_{\text {ref }}$ to be

$$
\vec{v}_{\text {ref }} \stackrel{\text { def }}{=} \vec{u}_{\infty}-\vec{u}_{S}
$$

Substituting (2.27), (2.41), and (2.42) in equation (2.40) we find after some manipulations an expression for the pressure coefficient:

$$
C_{p} \stackrel{\text { def }}{=} \frac{p-p_{\infty}}{\frac{1}{2} \rho_{\infty} v_{\text {ref }}^{2}}=1-\frac{u_{\text {rel }}^{2}}{v_{\text {ref }}^{2}}-\frac{2}{v_{\text {ref }}^{2}} \frac{\mathrm{D}_{S} \varphi_{m}}{\mathrm{D} t}+\frac{2}{v_{\text {ref }}^{2}} \vec{g} \cdot\left(\vec{x}-\vec{x}_{\mathrm{ref}}\right),
$$

where $u_{\text {rel }}^{2}=\vec{u}_{\text {rel }} \cdot \vec{u}_{\text {rel }}$ and $v_{\text {ref }}^{2}=\vec{v}_{\text {ref }} \cdot \vec{v}_{\text {ref }}$. The total force vector and moment vector are determined by integrating the pressure distribution over the surface of the configuration (see Figure 2.6):

$$
\begin{aligned}
\vec{F}(t) & =-\iint_{S} p \bar{n} d S, \\
\vec{M}(t) & =\iint_{S} p \bar{n} \times \vec{r} d S,
\end{aligned}
$$

where $\vec{r}$ is the position vector from a moment reference point to the surface, and $p$ the local pressure acting on the surface of the body in the direction towards the surface.

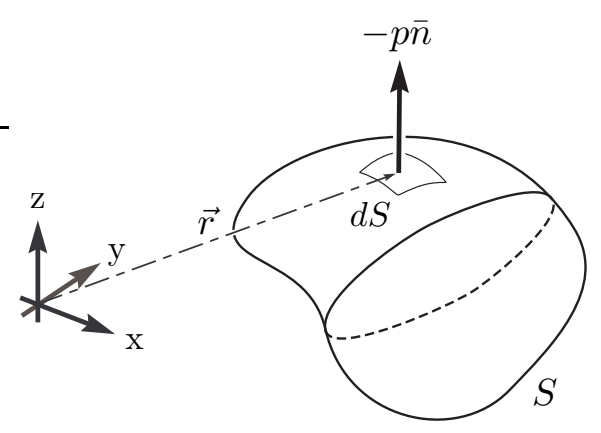

Figure 2.6: Surface pressure integration. 
For configurations in motion the rate of work done by the distributed forces over the surface is the instantaneous power $P$ and can be expressed as

$$
P(t)=-\iint_{S} p \vec{u}_{S} \cdot \bar{n} d S .
$$

This power is extracted from the fluid and is for rigid wind turbine rotors equal to the power at the rotor shaft. Expressed in torque $\vec{M}$ and angular velocity $\vec{\Omega}$ the instantaneous power is in this case

$$
P(t)=\vec{M} \cdot \vec{\Omega}=-\iint_{S} p \bar{n} \cdot(\vec{\Omega} \times \vec{r}) d S,
$$

where $\vec{r}$ is the position vector from a reference point on the rotor axis to the surface. 


\section{Low Order Panel Method}

\subsection{Introduction}

For general wind turbine configurations with components in arbitrary motion there is no analytical solution to the unsteady $3 \mathrm{D}$ potential flow equations (2.30) described in the preceding chapter. We will discretize the equations with a low-order panel method ${ }^{1}$ using the definitions for the perturbation potentials for surface dipole distributions and source distributions and numerically solve for a finite number of degrees of freedom. From the solution we can then determine the velocity vector field and obtain the pressure distribution. From the surface pressure distribution we compute the forces and moments acting on the configuration.

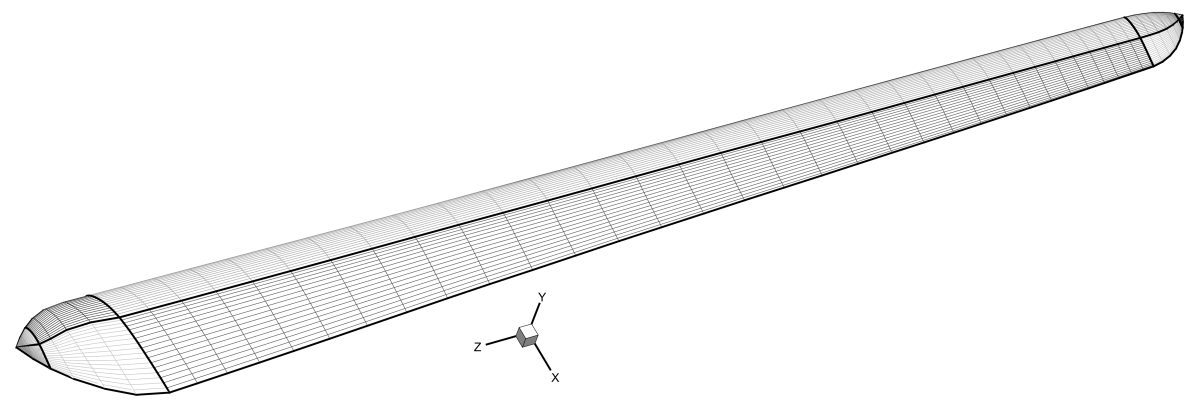

Figure 3.1: Rotor blade surface subdivided into a number of patches with a structured surface grid of panels.

The key property of the panel method is that the solution of the flow equations in 3D space is fully determined by the solution on the surface of the configuration only. This makes the geometric modeling of the problem a relatively simple task. The geometry will be described in terms of bodies and wakes each consisting of structured patches of surface grids, the so called panels in the method. Figure 3.1 shows an example paneling of a

\footnotetext{
${ }^{1}$ The classical term panel method is used here instead of the in scientific literature generally used term Boundary Element Method as that can easily be confused with the Blade Element Momentum methods still in use in the wind energy aerodynamics community that share the same acronym BEM.
} 
wind turbine blade.

In a low order panel method the error in the solution is of $\mathcal{O}(h)$ and thus linearly dependent on a characteristic panel size $h$. We follow the discretization introduced by Morino and Kuo [77] and later popularized by Maskew [73]. More details can be found in the book of Katz and Plotkin [63]. Dirichlet boundary conditions are enforced at a finite number of discrete points, so-called collocation points that are located just below the surface of the body panel midpoints. Some characteristics of the approach followed are:

- Body and wake surface geometries are represented by structured grids of quadrilateral panels and the arbitrary translational and/or rotational motion of (parts of) the geometry is specified in a hierarchical setup described in Section 3.2.

- Each panel on a body or wake patch is assigned a constant strength dipole distribution and for body patches the panels are also assigned a constant strength source distribution (Section 3.3).

- A simple Euler time integration scheme is used to advance the growth and deformation of the wake during each time step.

- The perturbation velocity vectors at the panel corner points are determined by a $2 \mathrm{D}$ version of the Gradient Theorem, see Section 3.4.1.

- The aerodynamic force and moment on each panel are determined through the integration of the surface pressure distribution, obtained by a bi-linear interpolation of all contributions in the Bernoulli equation that define the pressure. Summation then yields the force and moment acting on all geometric components (Section 3.4.2).

- The implemented $\mathcal{O}(h)$ low order panel method is validated with some test cases for tri-axial ellipsoids. For these cases the method is demonstrated to be of $\mathcal{O}\left(h^{2}\right)$ in the velocity potential, with $h$ a characteristic panel size.

\subsection{Geometry}

The surface of the configuration is subdivided into one or more patches which are discretized in a structured surface grid. The grid cells on the surface of the configuration are in the boundary integral discretization called 
panels, hence the name for this numerical discretization method. An example rotor blade surface subdivided into a number of patches, each patch consisting of a grid of panels, is shown in Figure 3.1.

Each patch consist of an array of nodes in $i$ and $j$ direction (see Figure 3.2a) which determine the corner points of the panels. The direction of the vector normal to the surface is defined to be in the direction of the right-hand rule cross product $\bar{n} \equiv \bar{i} \times \bar{j}$ as is indicated in Figure $3.2 \mathrm{~b}$.

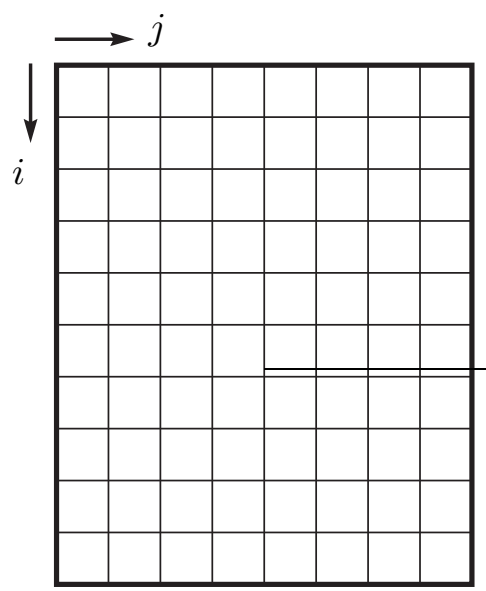

(a) Patch: Ordering nodes and panels in a structured grid using indices $i$ and $j$ with corresponding directions $\bar{i}$ and $\bar{j}$. Here the normal vector points towards the reader.
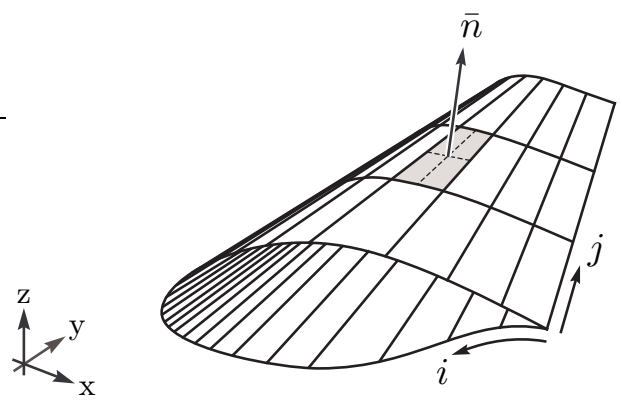

(b) The unit normal vector $\bar{n}$ is assumed to point into the flow domain and is deduced from the right-hand rule vector cross product of the $i$ and $j$ index related directions: $\bar{n} \equiv \bar{i} \times \bar{j}$

Figure 3.2: Node ordering and panel normal vectors in a patch.

The direction of the normal vector at each panel midpoint is determined by the cross product of the vectors through diagonally opposite panel corner points.

$$
\vec{n}=\left(\vec{x}_{i+1, j}-\vec{x}_{i, j+1}\right) \times\left(\vec{x}_{i+1, j+1}-\vec{x}_{i, j}\right),
$$

which is normalized to give the unit vector $\bar{n}_{\text {mid }}=\vec{n} /|\vec{n}|$. The panel area is approximated by a flat, non-curved panel surface representation and equal to $|\vec{n}| / 2$. The unit normal vectors at the panel corner points are determined by the normalized version of

$$
\vec{n}_{i, j}=\left(\vec{x}_{i+1, j}-\vec{x}_{i-1, j}\right) \times\left(\vec{x}_{i, j+1}-\vec{x}_{i, j-1}\right) .
$$




\subsubsection{Solid Body Transformations}

For the arbitrary motion of the geometry we use the same technique as described in some earlier work (Van Garrel [35]). Each time step the new constellation of a user specified geometry is determined. Solid body rotations and translations are performed through the use of transformation matrices expressed in a homogeneous coordinate system. Compound transformations that combine a hierarchy of body rotations and translations of multiple connected bodies can then be represented as matrix products (see Faux and Pratt [34]). These $4 \times 4$ matrices operate on homogeneous coordinates which are composed of the 3 components of a Cartesian vector supplemented with a $4^{\text {th }}$ component equal to 1 . A position vector $(x, y, z)^{\mathrm{T}}$ is thus extended in homogeneous form to $(x, y, z, 1)^{\mathrm{T}}$.

The rotation matrix $\mathbf{R}$ for rotation of a position vector $\vec{x}$ about a general axis through the origin with direction $\bar{v}=\left(v_{1}, v_{2}, v_{3}\right)^{\mathrm{T}}$ through an angle $\theta$, interpreted in a right-hand rule sense, reads

$$
\mathbf{R}=\left(\begin{array}{llll}
v_{1} v_{1} d+c & v_{1} v_{2} d-v_{3} s & v_{1} v_{3} d+v_{2} s & 0 \\
v_{2} v_{1} d+v_{3} s & v_{2} v_{2} d+c & v_{2} v_{3} d-v_{1} s & 0 \\
v_{3} v_{1} d-v_{2} s & v_{3} v_{2} d+v_{1} s & v_{3} v_{3} d+c & 0 \\
0 & 0 & 0 & 1
\end{array}\right)
$$

where

$$
\begin{aligned}
& c=\cos \theta \\
& s=\sin \theta \\
& d=1-\cos \theta,
\end{aligned}
$$

and

$$
\bar{v} \cdot \bar{v}=1 .
$$

The rotation of a position vector $\vec{x}$ is now accomplished by a matrix multiplication that gives a new position vector $\vec{x}_{\text {new }}=\mathbf{R} \vec{x}$.

Similarly, the translation of a position vector $\vec{x}$ can be performed through multiplication with the translation matrix $\mathbf{T}$ such that $\vec{x}_{\text {new }}=\mathbf{T} \vec{x}$. The translation matrix reads

$$
\mathbf{T}=\left(\begin{array}{llll}
1 & 0 & 0 & t_{1} \\
0 & 1 & 0 & t_{2} \\
0 & 0 & 1 & t_{3} \\
0 & 0 & 0 & 1
\end{array}\right)
$$

where $\left(t_{1}, t_{2}, t_{3}\right)^{\mathrm{T}}$ is the Cartesian translation vector.

The transformation matrix for a translation in the reverse direction $-\left(t_{1}, t_{2}, t_{3}\right)^{\mathrm{T}}$ is the inverse $\mathbf{T}^{-1}$ of matrix $\mathbf{T}$. Rotation about a general axis not through 
the origin can be performed by a compound transformation matrix $\mathbf{A}$, which is composed of a translation of the axis to the origin by $\mathbf{T}$, a rotation $\mathbf{R}$, and a translation $\mathbf{T}^{-1}$ back to the original position:

$$
\vec{x}_{\text {new }}=\mathbf{T}^{-1}(\mathbf{R}(\mathbf{T} \vec{x}))=\mathbf{A} \vec{x}
$$

For a set of position vectors $\vec{x}$ it is more efficient to compute the compound transformation matrix $\mathbf{A}$ first, and perform the matrix multiplication with all position vectors next.

The generic setup of a geometry and the (user specified) solid body rotations and translations of the individual parts is implemented through the use of so-called markers as was done in [35]. Each marker is defined by a position vector and the oriented vectors of a component specific orthogonal coordinate system. The basic procedure will be explained with the help of Figure 3.3 that shows a rotor blade and two fictitious bodies with their associated markers: the nacelle/tower combination, and the rotor hub for a three-bladed wind turbine. Fictitious bodies are introduced to associate one or more markers with each of them. To assemble all the components (specified in their component-specific coordinate system) in the correct constellation some of the markers will be pairwise linked.

In our example the marker at the blade root is linked to one of the exterior markers at the hub, and the hub axis marker is linked to the nacelle/tower marker. In addition each of the two linked markers is assigned a role: one is set to be parent and one is set to be child. Each pairwise link defines a transformation matrix that makes that the markers of the transformed bodies have identical position and orientation, and the two roles determine which one of the two components is transformed and which one is leading. In our example the nacelle/tower component is the parent of the hub component which in turn is the parent of the blade component. Through the parent-child hierarchy the transformation matrices are propagated from parent down to the final child components meanwhile compounding the transformation matrices that will be applied to the original definition of the component geometry.

To enable a time dependent constellation of the components, the markers are allowed to rotate and translate relative to their associated component during the simulation. This marker motion is specified by the user and defined in the original coordinate system of the marker at hand. In our example in Figure 3.3 we would rotate the nacelle/tower marker with a time-dependent angle along the rotor axis and could specify a rotor blade pitch action by a rotation along the radial direction of the hub marker to which the blade is connected. A specified marker translation could be useful for example to take the effects of tower top displacements into account. 


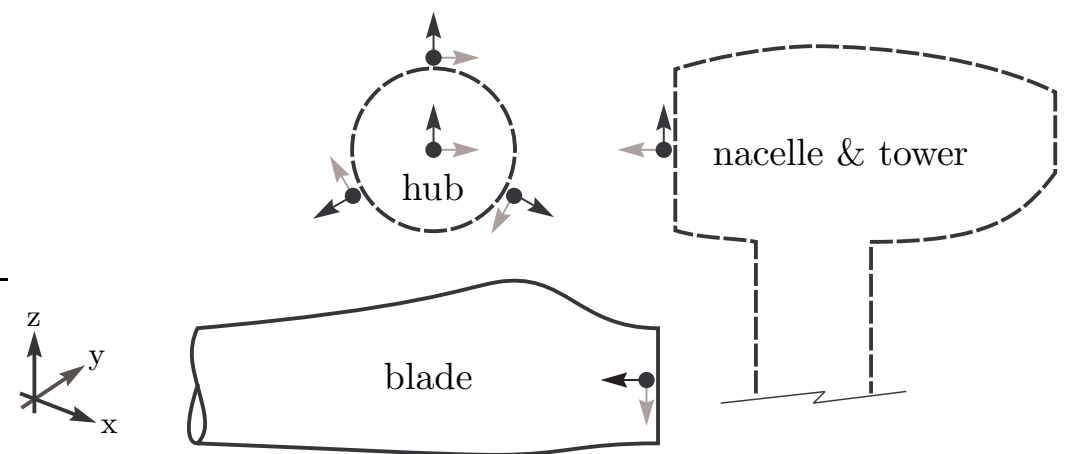

marker:

Figure 3.3: Setup of wind turbine components through the use of markers.

\subsection{Aerodynamic Influence Coefficients}

With the components of the configuration in the correct constellation the boundary integral equations (2.30) are discretized as a low order panel method (see Hoeijmakers [58], Katz and Plotkin [63]) using the definitions for the perturbation potentials for dipole distributions (2.10) and source distributions (2.11). In the low order approach each body panel $j$ carries a constant source $\sigma_{j}$ of known strength and a constant dipole $\mu_{j}$ singularity distribution of unknown strength. Boundary condition (2.16) is enforced in each panel collocation point $\vec{x}_{i}$, located just below the panel's surface midpoint, and determines through (2.21) the panel source strength $\sigma_{j}$.

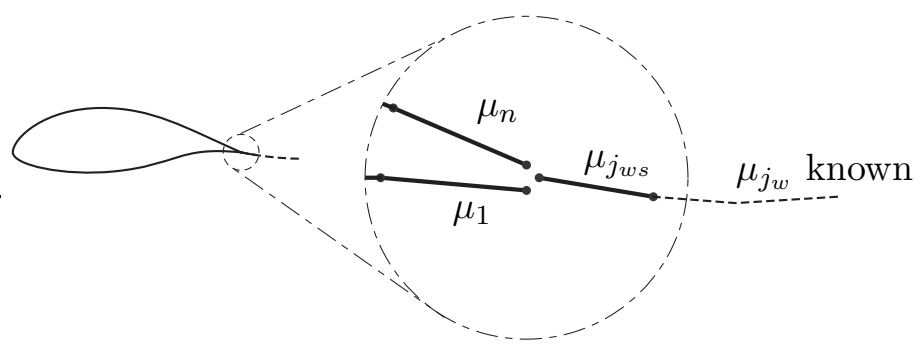

Figure 3.4: Numerical Kutta condition.

The $N_{w}$ downstream wake panels $j_{w}$ that are not attached to a body have 
a dipole strength $\mu_{j_{w}}$ known from previous time steps (see Figure 3.4). For wake panels at the start of the wake attached to two body panels the strength is determined by the Kutta condition (2.31), which in discretized form reads

$$
\mu_{j_{w s}}=\mu_{n}-\mu_{1}
$$

In the current implementation of the panel method the Kutta condition is not included as a separate equation. Instead, the dipole influence coefficients of the wake panels that are attached to the rotor blade are added to, or subtracted from, the influence coefficients of the trailing edge panels to which they are attached, depending on the orientation of the panel normals. In the case that the geometry of the rotor and the immediate wake panels attached to the trailing edges of the rotor blades do not deform and can be considered a "solid body", the associated matrix of dipole influence coefficients is constant throughout the simulation. In the solution of the system of equations this property is exploited.

The discrete set of equations in $N_{b}$ collocation points to solve for the $N_{b}$ unknown dipole strengths $\mu_{j}$ that results is

$$
\sum_{j} A_{i j} \mu_{j}=-\sum_{j} B_{i j} \sigma_{j}-\sum_{j_{w}} A_{i j_{w}} \mu_{j_{w}}, \quad \text { for } \quad i=1 . . N_{b},
$$

where $A_{i j}$ and $B_{i j}$ are aerodynamic influence coefficients defined by

$$
\begin{aligned}
& A_{i j}=\frac{-1}{4 \pi} \iint_{S_{j}} \frac{\bar{n}_{j} \cdot \vec{r}}{r^{3}} d S, \\
& B_{i j}=\frac{-1}{4 \pi} \iint_{S_{j}} \frac{1}{r} d S,
\end{aligned}
$$

in which

$$
\vec{r}=\vec{x}_{i}-\vec{y}, \quad r=|\vec{r}|, \quad \text { and } \quad \vec{y} \in S_{j}
$$

The resulting set of equations for the determination of the dipole strengths on the body can be solved through a conventional LU decomposition direct method or through a (restarted) GMRES iterative solution method as introduced by Saad and Schulz [96]. The direct method has a computational cost of $\mathcal{O}\left(N^{3}\right)$ that has to be performed only once if the matrix entries for the unknowns on the left hand side of equation (3.8) do not change during the simulation. In that case the solution for each subsequent right-hand side can be obtained at the cost of $\mathcal{O}\left(N^{2}\right)$. The iterative Krylov method has the advantage of having a computational cost of $\mathcal{O}\left(N^{2}\right)$ for a fixed number of matrix-vector multiplications, a cost that could be lowered even 
further with a multilevel multi-integration scheme such as described in the next chapter. For a clear treatise on Krylov methods for systems of linear equations the reader is referred to Van der Vorst [113].

The aerodynamic influence coefficients for the velocity induced by each panel carrying a constant dipole or a source distribution follow the definitions in (2.38) and (2.39) and, using the definitions in (3.11), read

$$
\begin{aligned}
\vec{C}_{i j} & =\frac{-1}{4 \pi} \int_{\partial S_{j}} \frac{\vec{r}}{r^{3}} \times d \vec{l}, \\
\vec{D}_{i j} & =\frac{1}{4 \pi} \iint_{S_{j}} \frac{\vec{r}}{r^{3}} d S .
\end{aligned}
$$

For the velocity at a point $\vec{x}_{i}$ anywhere in the domain a summation of the contributions from all $N_{b}$ panels on the body and all $N_{w}$ panels in the wake is needed:

$$
\vec{u}\left(\vec{x}_{i}\right)=\sum_{j} \vec{C}_{i j} \mu_{j}+\sum_{j} \vec{D}_{i j} \sigma_{j}+\sum_{j_{w}} \vec{C}_{i j_{w}} \mu_{j_{w}} .
$$

The velocity at a point on the surface of a body component is much cheaper to obtain and will be discussed in Section 3.4.1.

To account for the growth of the wake, each time step new panels are shed from the trailing edge of lifting components. For the deformation of the wake in time we determine the velocity vector at all downstream wake panel corner points through (3.14) and integrate equation (2.35) over a small time interval with a simple Euler scheme. Each wake panel maintains its dipole strength throughout the simulation as stated by equation (2.36). For wind turbine rotors the number of wake panels $N_{w}$ exceeds the number of panels $N_{b}$ on the rotor blades by a factor 10 easily and thus most of the computational cost for the wake deformation stems from the wake panel dipole distributions $\mu_{j_{w}}$ themselves. The work involved in determining the velocities at the corner points of $N_{w}$ wake panels by evaluation of $N_{w}$ wake panel contributions is of $\mathcal{O}\left(N_{w}^{2}\right)$.

\subsubsection{Dipole Potential and Velocity}

The velocity potential induced at point $\vec{x}$ by a panel with surface $S_{j}$ and dipole distribution $\mu(\vec{y})$ is given by

$$
\varphi_{\mu}(\vec{x})=\frac{-1}{4 \pi} \iint_{S_{j}} \mu \frac{\bar{n} \cdot \vec{r}}{r^{3}} d S,
$$


where

$$
\vec{r}=\vec{x}-\vec{y}, \quad r=|\vec{r}|, \quad \text { and } \quad \vec{y} \in S_{j} .
$$

The usual approach taken in low-order panel methods is to use a flat surface approximation for the panel geometry, for which analytical results exists for the integral in equation (3.15) (see Katz and Plotkin [63], Newman [80]). In general, flat panels, however, lead to gaps between the panels in the surface approximation of for a curved surface, that grow larger with increasing surface curvature and twist. Especially for panels on a highly deformed wake surface this flat panel approximation is inadequate. The approach taken here is to use a bilinear representation for the panel geometry (see Figure 3.5) which gives a better surface approximation and avoids gaps altogether.

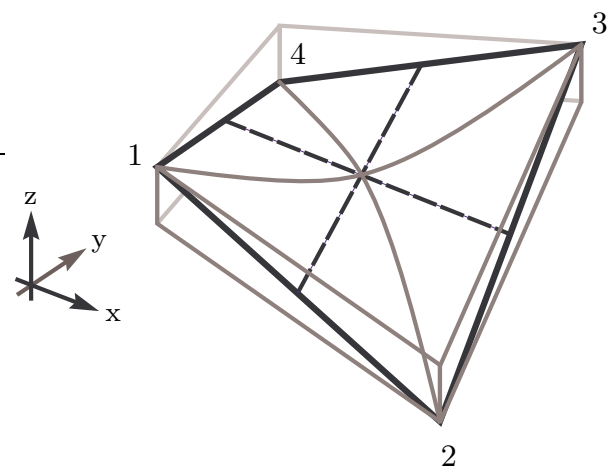

Figure 3.5: A warped quadrilateral panel.

For a unit strength dipole distribution $(\mu=1)$ the integral in equation (3.15) is equal to the solid angle and can be determined by the projection of the warped panel on a sphere with unit radius, and with the evaluation point $\vec{x}$ as its center (see Figure 3.6). The solid angle is then the ratio between the projected panel area and the surface area of the sphere. We use the result that the area of an $n$-sided polygon on a sphere with unit radius is equal to the sum of the included angles minus $(n-2) \pi$ :

$$
\text { area }=-(n-2) \pi+\sum_{i=1}^{n} \beta_{i} .
$$

For an evaluation point that is located outside the box that can be constructed around a highly twisted panel, see Figure 3.5, the resulting formulas can be implemented in a straightforward fashion. When the evaluation 


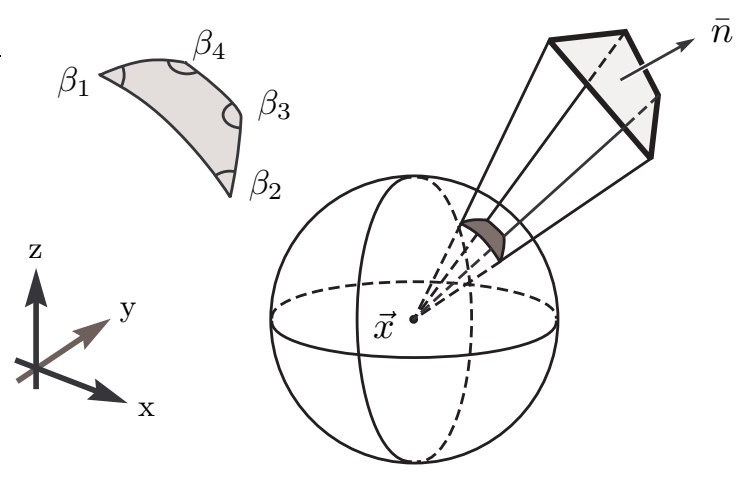

Figure 3.6: The solid angle is the ratio between the area of the projected panel and the surface area of the sphere with the evaluation point as its center. The area of the projected quadrilateral can be determined from its included angles $\beta_{i}$.

point is within this box, care has to be taken for the jumps in the $\arctan ()$ function at the surface. The result then depends on which side of the surface the evaluation point is located. There is no ambiguity on which side a point is located for triangular panels and the implementation of the dipole potential influence coefficient can be kept simpler.

To determine the induced velocity due to a constant strength dipole distribution on a warped, bilinear panel geometry we make use of the equivalence of the contribution of such a distribution with that of a ring vortex consisting of line elements defined by the perimeter of the panel (see Hess [52], Hoeijmakers [58], Katz and Plotkin [63]). The velocity of a vortex line element with vortex strength $\Gamma=\mu$ is also known as the Biot-Savart law and reads

$$
\vec{u}_{\Gamma}(\vec{x})=\frac{-1}{4 \pi} \int \Gamma \frac{\vec{r} \times d \vec{l}}{r^{3}} .
$$

For a straight line element with constant vortex strength $\Gamma$ its contribution to the integral for the ring vortex velocity field can be expressed analytically as

$$
\vec{u}_{\Gamma}(\vec{x})=\frac{\Gamma}{4 \pi} \frac{\left(r_{1}+r_{2}\right)\left(\vec{r}_{1} \times \vec{r}_{2}\right)}{r_{1} r_{2}\left(r_{1} r_{2}+\vec{r}_{1} \cdot \vec{r}_{2}\right)} .
$$

In this expression $\vec{r}_{1}$ and $\vec{r}_{2}$ are the position vectors from the vortex line start and end positions $\vec{x}_{1}$ and $\vec{x}_{2}$ to the evaluation point $\vec{x}$, respectively (see Figure 3.7). The $r_{1}$ and $r_{2}$ terms are respective distances and the orientation of the vortex line element is determined by the panel normal vector in a right-hand rule sense. 


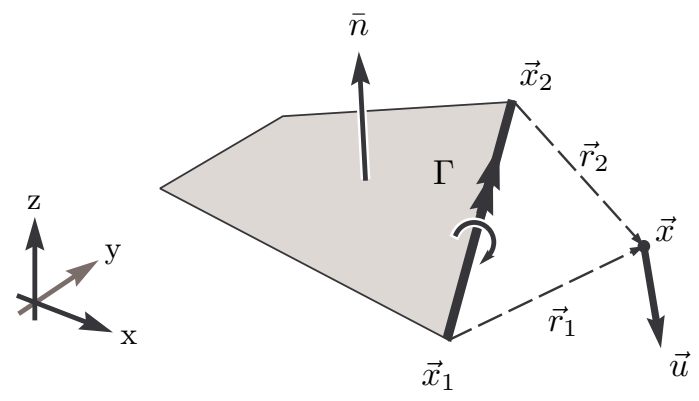

Figure 3.7: Ring vortex line elements.

Summation of the contributions of the panel line elements for $\Gamma=1$ and $\vec{x}=\vec{x}_{i}$ gives the induced velocity aerodynamic influence coefficient $\vec{C}_{i j}$.

\subsubsection{Source Potential and Velocity}

For flat panels the integrals for the velocity potential $B_{i j}$ in $(3.10)$, and the velocity vector $\vec{D}_{i j}$ in $(3.13)$ at a point $\vec{x}_{i}$ induced by a panel with constant strength source distribution can be evaluated exactly. For these analytical results the reader is referred to Katz and Plotkin [63] and Newman [80].

\subsection{Post-processing}

The solution of the system of equations (3.8) gives the dipole strengths for the panels on the configuration and further post-processing is required to obtain the forces and moments acting on the configuration.

\subsubsection{Velocity Vector Field}

To obtain the total velocity $\vec{u}$ at the surface of the configuration, one of the terms in equation (2.26) that has to be determined is the surface gradient of the dipole strength $\nabla_{S} \mu$. This is accomplished with the help of the Gradient Theorem (see Appendix A):

$$
\iint_{S} \nabla_{S} \mu d S=\int_{\partial S} \mu \bar{\nu} d l,
$$


where $\bar{\nu}$ is the unit outward vector normal to the contour and tangential to the surface (see Figure 3.8):

$$
\bar{\nu}=\frac{\vec{\tau} \times \bar{n}}{|\vec{\tau} \times \bar{n}|}
$$

in which $\vec{\tau}$ is the vector tangential to the contour and $\bar{n}$ the local unit normal vector. This gives an expression for the average dipole surface gradient inside contour $\partial S$

$$
\overline{\nabla_{S} \mu}=\frac{1}{S} \int_{\partial S} \mu \bar{\nu} d l .
$$

In the current implementation the contour $\partial S$ is defined by the straight lines $\bar{\tau}$ through the collocation points of the four neighboring panels and normal vector $\bar{n}$ is set to the unit normal vector at the panel node which is constructed from the four nodes that connect to it, as Figure 3.8 shows. With the definition of the surface tangential vector $\bar{\nu}$ in equation (3.21) this gives automatically a surface gradient normal to $\bar{n}$. As a further approximation the perturbation velocity at the grid node due to the dipole distribution is assigned this average surface gradient.
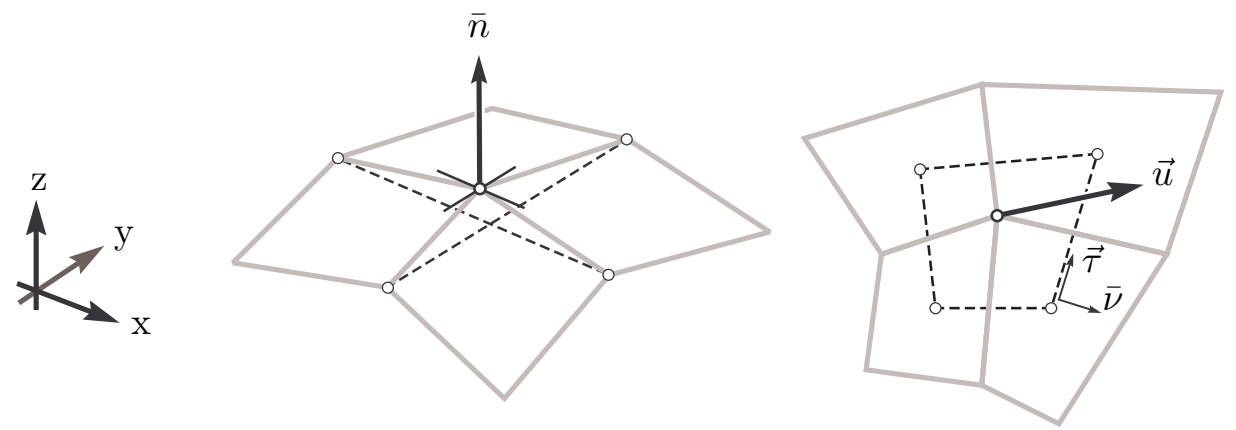

Figure 3.8: Definitions used in the contour integral to determine the surface perturbation velocity at a grid node due to a dipole distribution.

We can now determine the total and the relative velocity vector fields at the grid nodes of the configuration with the help of equations (2.26) and (2.28). In the current implementation the surface velocity field $\vec{u}_{S}$ due to the motion of the boundary is determined by a simple finite difference of the last three positions in time of the grid nodes. 


\subsubsection{Aerodynamic Forces and Moments}

To obtain the aerodynamic forces and moments on the configuration the contribution of each panel to the total is determined by numerical integration of the surface pressure distribution.

The velocity relative to the moving body $\vec{u}_{\text {rel }}$ in the boundary grid nodes is described by equation (2.28). To determine the pressure at the grid nodes through equation (2.43) we still need to obtain the time-dependent term $\frac{\mathrm{D}_{S} \varphi_{m}}{\mathrm{D} t}$. This is achieved by a finite difference formula that uses the results at each collocation point of the last three solutions in the time stepping scheme. A simple averaging of the time dependent terms of the neighboring panels is used to obtain its value at each grid node.

For each panel we set up a local non-orthogonal coordinate system $(\xi, \eta)$ and write for the contribution of panel $j$ to the total force vector and its local moment vector around the panel mid point

$$
\begin{gathered}
\vec{f}_{j}=-\iint_{S_{j}} p \bar{n} J d \xi d \eta, \\
\vec{m}_{j}=\iint_{S_{j}} p \bar{n} \times \vec{r} J d \xi d \eta
\end{gathered}
$$

where $\vec{r}=\vec{x}-\vec{x}_{\text {mid }}$ is a position relative to the panel midpoint $\vec{x}_{\text {mid }}$ with $\vec{x} \in S_{j}$, and the Jacobian of the coordinate transformation is defined by

$$
J=\left|\frac{d \vec{x}}{d \xi} \times \frac{d \vec{x}}{d \eta}\right| .
$$

These integrals are evaluated with a tensor product Gaussian quadrature rule in which the pressure is obtained in the Gauss points by a bi-linear interpolation of the velocity and time derivative term in the Bernoulli equation (2.43). Also the normal vector and the Jacobian of the transformation are interpolated linearly in each direction from the values at the panel corner points. The combination of all bi-linear functions contributing to the panel force (3.23) and moment (3.24) is a function of degree $\xi^{5} \eta^{5}$ at most and can be integrated exactly with a $3 \times 3$ Gaussian quadrature rule.

The rationale for the use of this higher order Gaussian quadrature rule instead of the more commonly used mid point quadrature in low order panel methods lies in the geometric nature of the problem. Modern wind turbines have very slender blades with length/chord ratios of 15 , and as a consequence most panels on the surface also have a high aspect ratio with the largest dimension in radial direction. In addition, the rotation of the 
blades makes that a linear variation of the velocity in radial direction and thus a quadratic variation of the pressure is the more common situation. These effects combined favor a higher order quadrature rule to achieve adequate accuracy.

\subsection{Verification}

Verification tests have been performed for the implemented panel method to determine if the computed results tends to the continuous solution of the mathematical problem. With the exception of the final test, all tests have been performed on a tri-axial ellipsoid with semi-axes $(4,2,1)$ as shown in Figure 3.9.

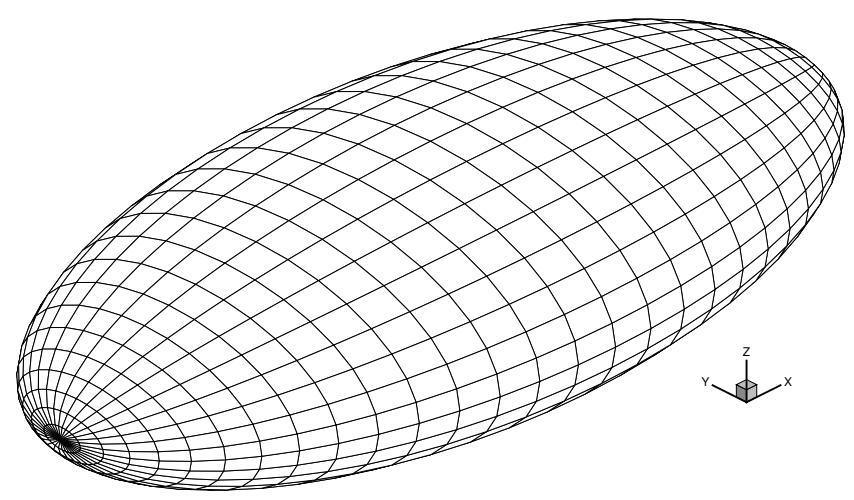

Figure 3.9: Tri-axial ellipsoid with semi-axes $(4,2,1)$ and $32 \times 32$ paneling.

We first test the correctness of the approximation of the geometry. Next we determine the solid angle at points interior to the ellipsoid body in order to verify the correctness of the implementation for the analytic expression of the integral for the dipole velocity potential (3.9). The introduction of an approximation for this integral is investigated and the order of accuracy of the panel method is determined.

The next set of tests makes use of two exact analytical solutions for inviscid, incompressible flow around tri-axial ellipsoids (see Appendix G). One exact solution is for a stationary ellipsoid in a uniform onset flow, and the other is for a tri-axial ellipsoid rotating around an axis through its center in flow at rest.

The final test is for a lifting wing with elliptic planform and very high aspect ratio. A Kármán-Trefftz (KT) airfoil section is used to define the 
cross sections of the wing. For this family of airfoils exact solutions in 2D inviscid incompressible flow are known.

The errors in the verification tests are based on the continuous $\mathrm{L}_{m}$-norm of a function $x$ over surface $S$ that reads

$$
\mathrm{L}_{m}(x)=\left(\frac{1}{S} \int_{S}|x|^{m} d S\right)^{1 / m} .
$$

The discrete versions of this definition for the norms used in this thesis are

$$
\begin{aligned}
\mathrm{L}_{1}(x) & =\frac{1}{n} \sum_{i=1}^{n}\left|x_{i}\right|, \\
\mathrm{L}_{2}(x) & =\left(\frac{1}{n} \sum_{i=1}^{n}\left|x_{i}\right|^{2}\right)^{1 / 2}, \\
\mathrm{~L}_{\infty}(x) & =\max _{i=1, n}\left|x_{i}\right|,
\end{aligned}
$$

where $\left|x_{i}\right|$ is the absolute value in case of scalar functions, and the length in case of vector functions.

\subsubsection{Geometry}

The convergence of the discretized geometry towards the exact ellipsoidal surface is investigated. The investigated geometric norms are based on the distance between the panel mid points $\vec{x}_{c}$, and the points $\vec{x}_{e}$ on the surface of the ellipsoid. The surface is discretized as a single-domain grid with flat panel geometry and poles along the x-axis as shown in Figure 3.9.

The vertex coordinates are obtained by scaling a sphere with a cosine distribution in $\mathrm{x}$-direction and an equi-angular distribution in azimuthal direction. The surface of the ellipsoid is discretized by an array of $n_{\xi}$ panels in azimuthal and $n_{\eta}$ panels in longitudinal direction, leading to a total of $\mathrm{N}=n_{\xi} n_{\eta}$ panels. In the geometric convergence study we set both $n_{\xi}$ and $n_{\eta}$ to $8,16,32, \ldots, 1024$.

The points $\vec{x}_{e}$ are obtained by projecting the collocation points $\vec{x}_{c}$ along the panel normal vector $\bar{n}_{c}$ onto the surface as described in Appendix G.1.

The variation of the error norms with the dimensionless parameter $\mathrm{L}_{\mathrm{ref}} / h$ is shown in Figure 3.10, where $h=\mathrm{L}_{\text {ref }} / n_{\eta}$ is a characteristic panel length and $\mathrm{L}_{\mathrm{ref}}=8$ is a reference length equal to the length of the configuration. As can be seen, the error is $\mathcal{O}\left(h^{2}\right)$ in the geometric norms as expected. It should be noted that the largest errors were invariably for panels near the 
poles on the $x$-axis in the grid. This indicates that we would have to refine the mesh in these regions of large surface curvature in order to obtain a more uniform distribution of the error.

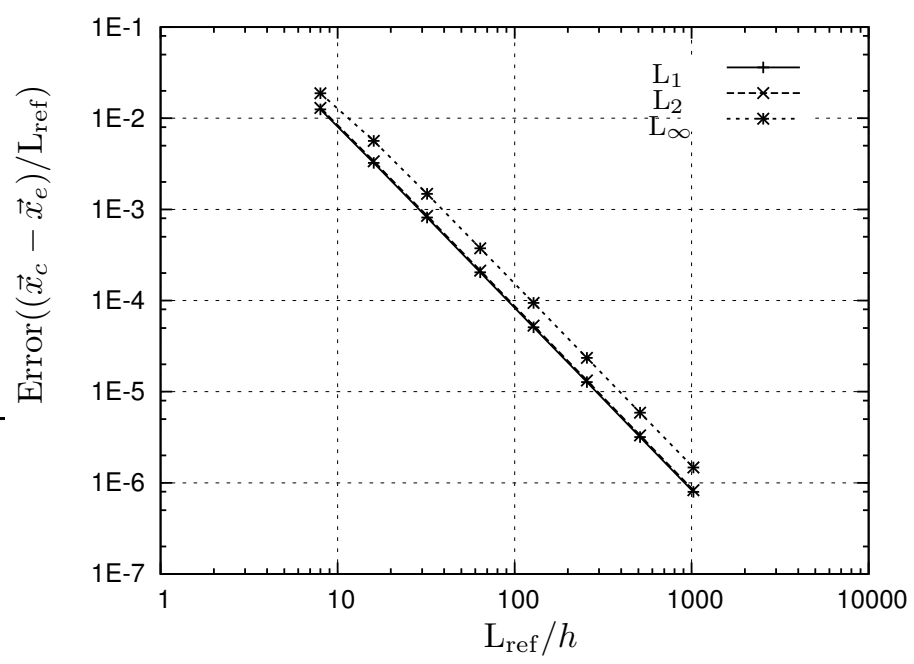

Figure 3.10: Convergence of distance between panel mid points and the surface of an ellipsoid with semi-axes $(4,2,1)$ as function of dimensionless characteristic panel size $\mathrm{L}_{\mathrm{ref}} / h$.

\subsubsection{Solid Angle}

The solid angle at collocation points just below the surface of an ellipsoid is determined. For internal points in closed volumes this solid angle has an exact value equal to the integral of the dipole perturbation velocity potential (2.10) for $\mu=4 \pi$. The correctness of the implementation for the analytic expression for the dipole velocity potential integral (3.9) is verified. Furthermore, the effect of an approximate formula for sufficiently distant panels is investigated. The particular setup we choose makes that the contribution from the analytic expression to the solid angle is exact and the error incurred in the induced velocity field is solely due to the point source approximations.

The solid angle $\vartheta$ at points $\vec{x}$ in the interior of a volume $V \in \mathbb{R}^{3}$ with bounding surface $\partial V$ the exact solution ${ }^{2}$ is $\vartheta(\vec{x} \in V)=4 \pi$. The surface

\footnotetext{
${ }^{2}$ The expression for the solid angle is obtained when $\Phi=1$ is substituted in (E.12).
} 
integral for the solid angle at a point $\vec{x}$ is

$$
\vartheta(\vec{x})=-\iint_{\partial V} \frac{\bar{n} \cdot \vec{r}}{r^{3}} d S,
$$

where

$$
\vec{r}=\vec{x}-\vec{y}, \quad r=|\vec{r}|, \quad \text { and } \quad \vec{y} \in \partial V,
$$

and $\bar{n}$ is the unit normal vector pointing out of the surface at $\vec{y}$. For flat panels an exact analytical expression exists for the integral in equation (3.30) and this allows us to factor out the discretization error due to an approximation for the panel integral.

The evaluation points are chosen to be the interior points approaching the panel midpoints in the limit. Using only the computationally expensive analytical expression, the solid angle was shown to be determined up to machine accuracy, independent of the number of panels.

In subsequent tests we use the costly exact integral expression for panels only in the near field of an evaluation point, for panels sufficiently far away we use a one-point quadrature approximation that is much cheaper to evaluate. These tests give an impression of the relation between the error introduced by the one-point quadrature for the panel integral, the panel size, the choice for the size of the near field, and the simulation time.

The transition from near field to far field is controlled by a near field parameter $n_{\text {nf }}$. Receiver points are considered to be in the near field of a panel when the distance between the panel midpoint and the receiver point is smaller than $n_{\mathrm{nf}}$ times the panel diagonal length. The surface of the ellipsoid is represented by $N=n_{\xi} \times n_{\eta}$ panels, with in the present case $n_{\xi}=n_{\eta}$. The reference length for the ellipsoid is $\mathrm{L}_{\mathrm{ref}}=8$, and the characteristic panel length $h$ is defined by

$$
h=\mathrm{L}_{\mathrm{ref}} / n_{\eta}
$$

We define a near field radius $r_{\mathrm{nf}}$ representative for the whole configuration as

$$
r_{\mathrm{nf}}=n_{\mathrm{nf}} h .
$$

In Figure 3.11 the error in the solid angle as function of a dimensionless near field radius is given for a range of surface grid resolutions. The selected error is the $\mathrm{L}_{2}$-norm of the relative differences with the exact value, that is, $(4 \pi-\vartheta) / 4 \pi$. For a constant near field radius it is clear that the error decreases with increasing number of panels on the surface. For this geometry a reasonable near field radius appears to be in the range of 10 
to 30 percent of the reference length. The data points on the lines are for $n_{\mathrm{nf}}=2^{k}$ with $k=0,1,2, \ldots$. All curves exhibit the largest error when $k=0$, that is when only the panels in the immediate neighborhood are evaluated with the exact integral formula. It is also clear from Figure 3.11 that for a given error level a finer surface paneling allows for a smaller near field radius $r_{\mathrm{nf}} / \mathrm{L}_{\text {ref }}$.

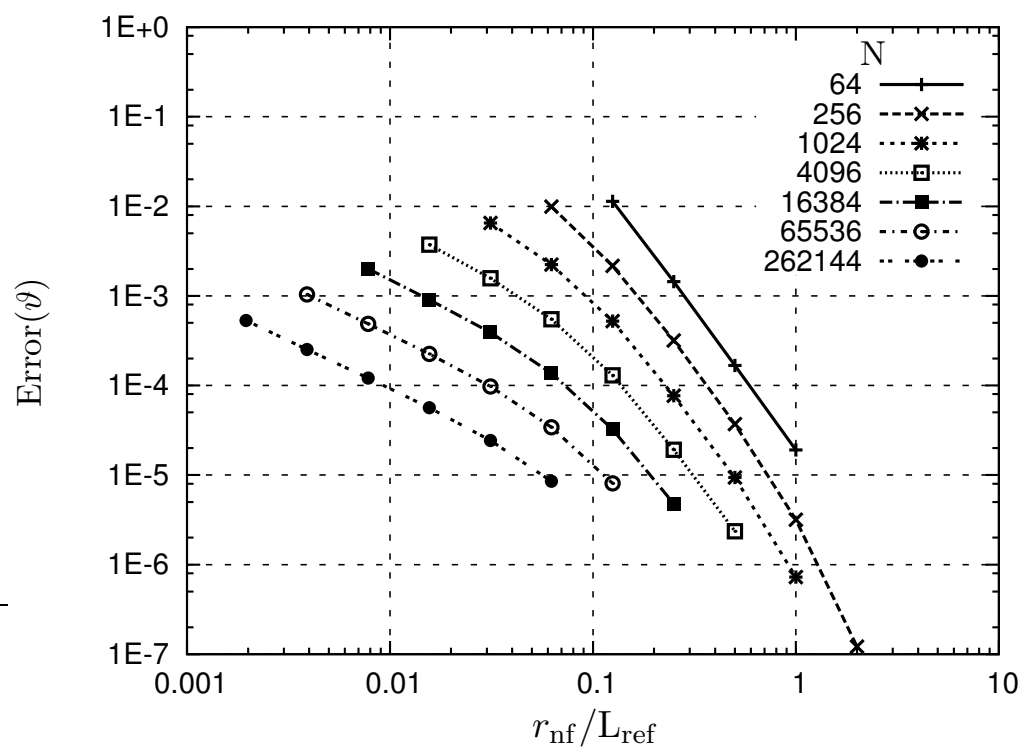

Figure 3.11: Relative $L_{2}$-norm error in the solid angle as function of near field radius for different surface grids of a $(4,2,1)$ semi-axes ellipsoid.

For a constant $n_{\mathrm{nf}}$ number of panels in the near field Figure 3.12 shows the behavior of the error as function of problem size $N$. For the smaller near field parameters the error in the computed solid angle is only mildly dependent on the problem size and decreases slightly for the largest problem sizes. Typical (relative) error levels are in the range $10^{-2} \leftrightarrow 10^{-5}$.

Figure 3.13 displays the relative error in solid angle as function of dimensionless reciprocal panel length for a range of near field radius values. Smaller panel sizes and larger near field sizes lead to a reduction in the error in the solid angle approximation. Moreover, the error is shown to be of $\mathcal{O}\left(h^{2}\right)$ for the whole range of fixed near field radius values considered.

For a panel method based on a Dirichlet boundary condition that uses flat panels with constant strength source and dipole distributions the theoretical order of the error for general problems is $\mathcal{O}(h)$ at most (see Hoeij- 


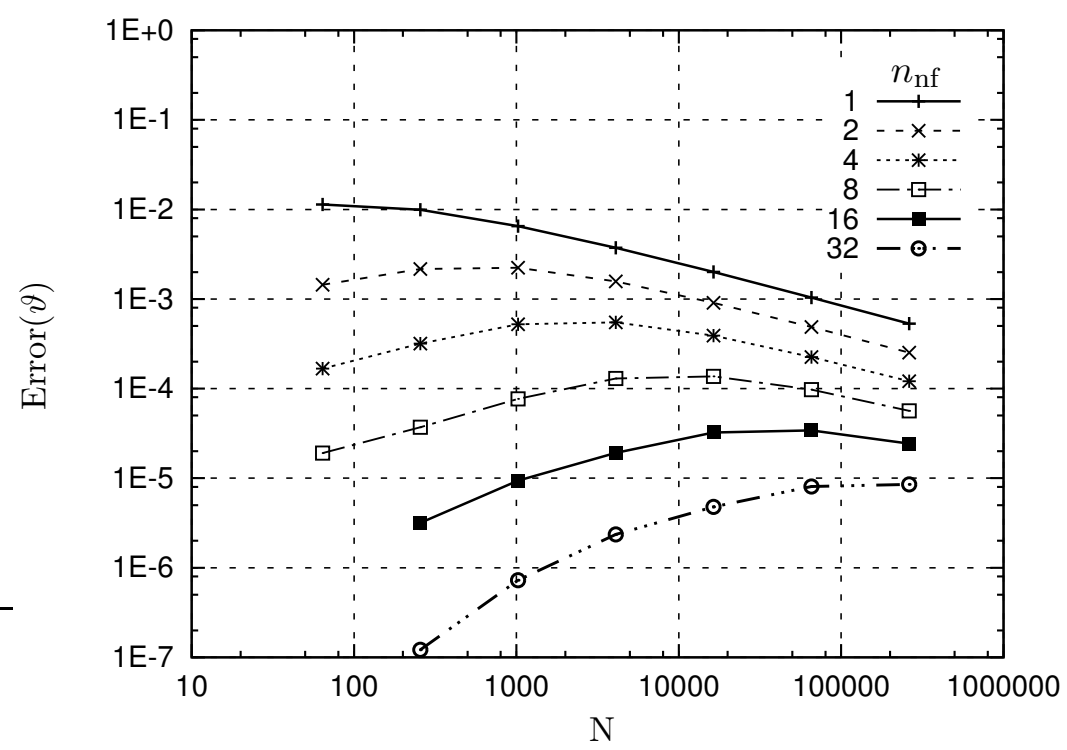

Figure 3.12: Relative $L_{2}$-norm error in the solid angle as function of problem size $N$ for different surface grids of a $(4,2,1)$ semi-axes ellipsoid.

makers [58], Katz and Plotkin [63], Oskam [88]). Such an $\mathcal{O}\left(h^{2}\right)$ super convergence was also noted by Bellamy-Knights et al. [8] for the simulation of $2 \mathrm{D}$ potential flow about an elliptical cylinder for which they assumed that the phenomenon was probably due to error cancellation occurring only for convex body shapes. For our solid angle test case the unit strength dipole distribution, the exact solution being constant, and the evaluation of the solid angle in the panel mid point could also be factors contributing to this occurrence of super convergence.

The variation of CPU time as function of dimensionless near field radius $r_{\mathrm{nf}} / \mathrm{L}_{\mathrm{ref}}$ is given in Figure 3.14. As expected, for a constant near field radius the CPU time for the computation of the solid angle increases with increasing total number of panels. It should be noted that in all cases the CPU time for the computation of the solid angle is dominated by the computation of the panel integrals and only for a small part (around 5\%) by the matrix vector multiplication. For a given number of panels, an increasing size of the near field leads to larger CPU times due to the increase in the costly analytic integral evaluations. This increase in CPU time slows down when some receiver points start to lie in the near field of all panels and a further increase of the near field size does not have an effect anymore. The significance of the results shown in Figures 3.13 and 3.14 is that for 


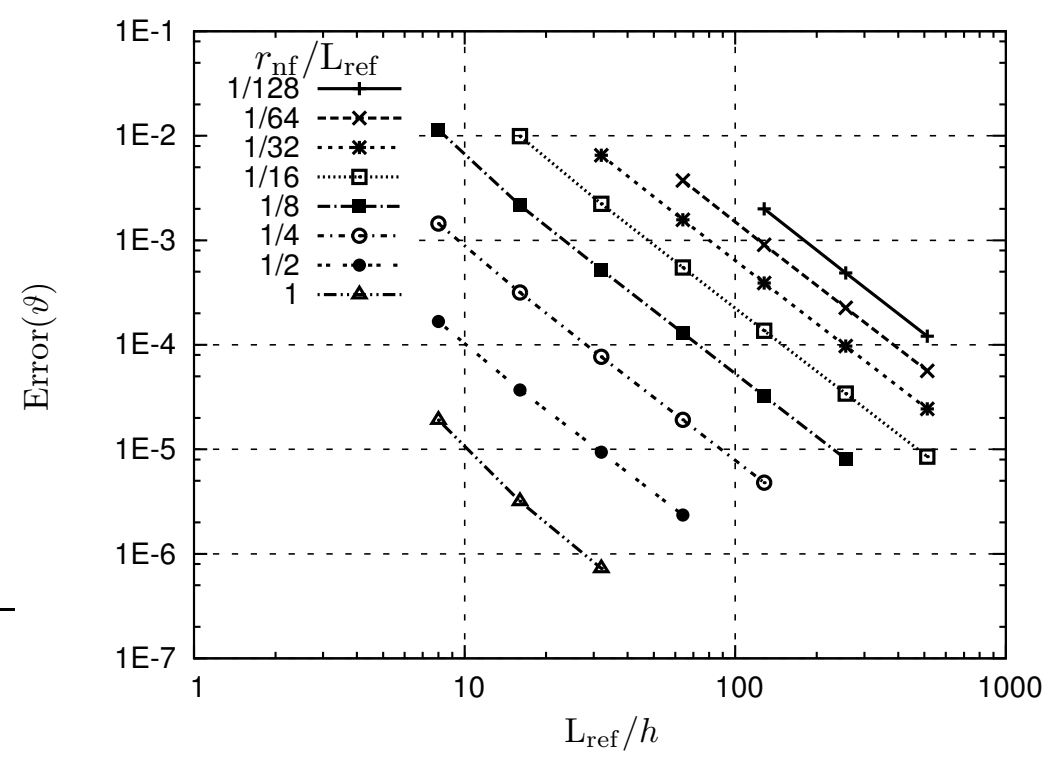

Figure 3.13: Relative $L_{2}$-norm error in the solid angle as function of reciprocal panel size for different near field radii for a $(4,2,1)$ semi-axes ellipsoid.

problems with large numbers of panels and relatively small near field radius it should be possible to speed up the evaluation of the point source contributions by the introduction of a fast multilevel scheme while still keeping the error $\mathcal{O}\left(h^{2}\right)$ through the use of appropriate polynomial interpolation orders as will be demonstrated in Chapter 4.

Figure 3.15 shows CPU time to be of $\mathcal{O}\left(N^{2}\right)$, a quadratic function of the total number of panels for every (fixed) near field radius. The CPU time for the case of only exact panel integral evaluations is much higher than the cases in which mostly point approximations are used. 


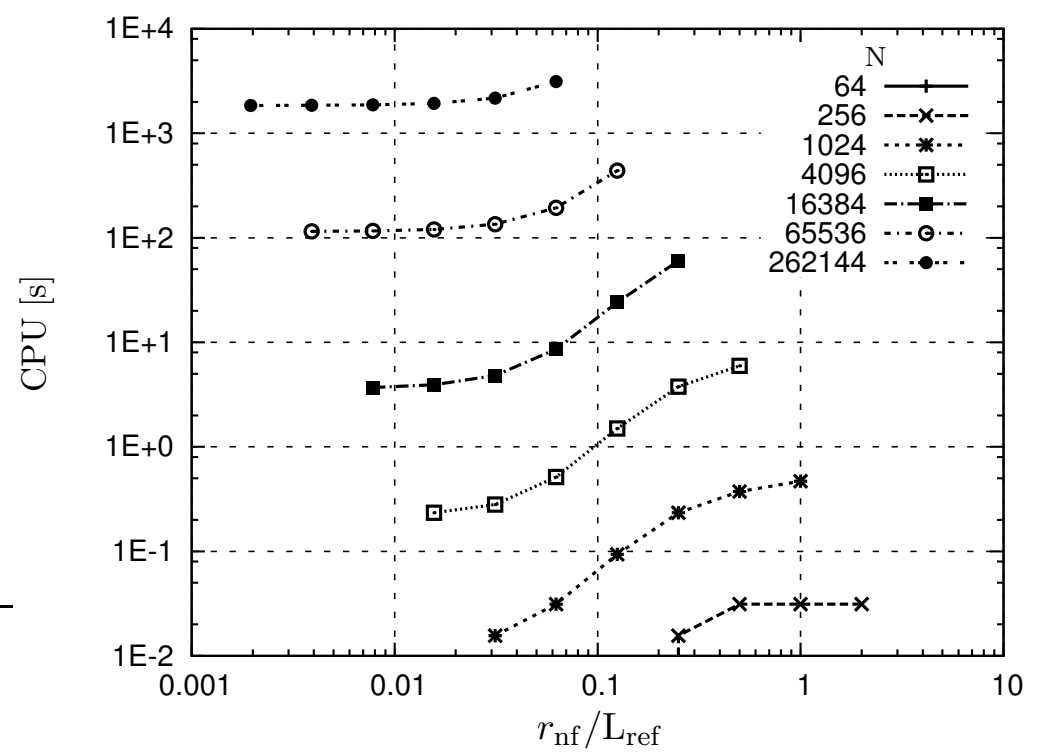

Figure 3.14: Solid angle evaluation CPU time as function of near field radius $r_{\mathrm{nf}} / \mathrm{L}_{\mathrm{ref}}$ for different surface grids of a $(4,2,1)$ semi-axes ellipsoid.

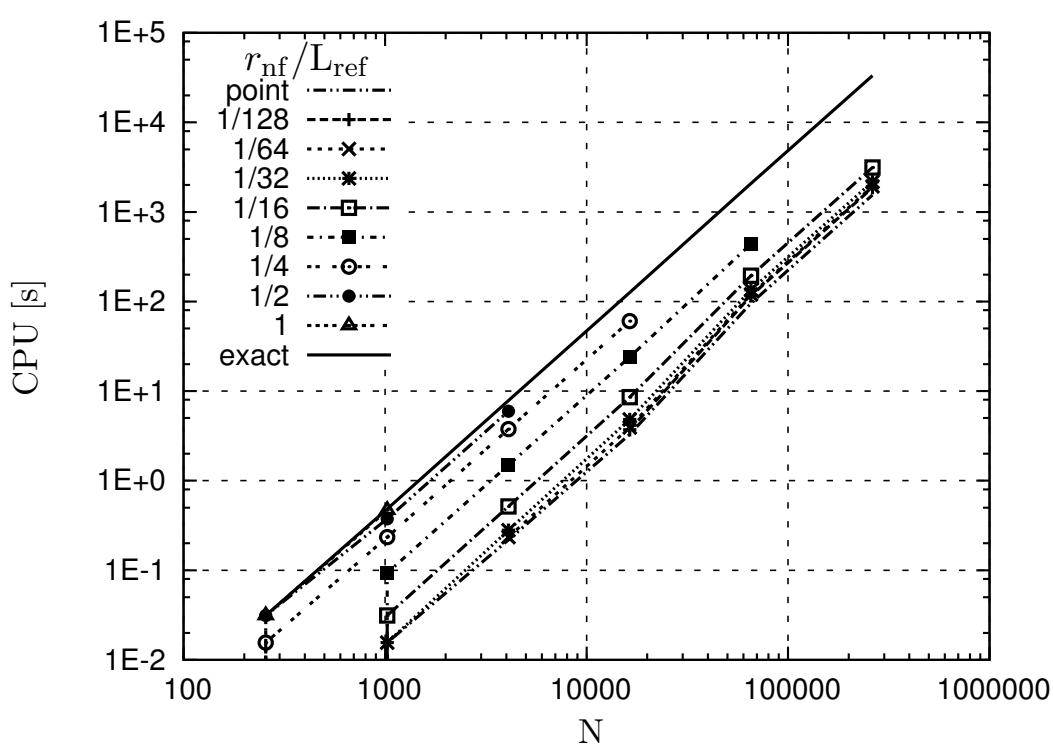

Figure 3.15: CPU time for solid angle evaluation as function of number of panels for different near field radii for a $(4,2,1)$ semi-axes ellipsoid. 


\subsubsection{Ellipsoid in Uniform Onset Flow}

For tri-axial ellipsoids in uniform, incompressible, inviscid onset flows exact solutions are available for the velocity potential distribution at the surface (see Appendix G.2). This makes it possible to verify the implementation of the panel method for the correct setup of the system of equations, the solution of the system of equations, and the application of post-processing steps necessary to obtain the surface velocity distribution and surface pressure distribution. Verification tests are performed for an ellipsoid with semi-axes $(4,2,1)$ for onset flows along each of the three axes. The ellipsoid surface is discretized by $N=n_{\xi} \times n_{\eta}$ panels where $n_{\xi}=n_{\eta}$.

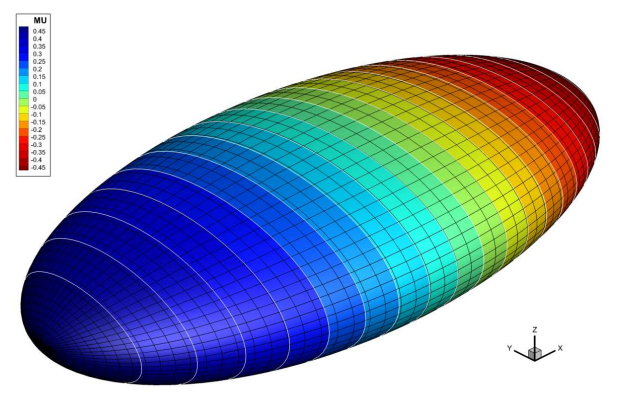

(a) Dipole strength.

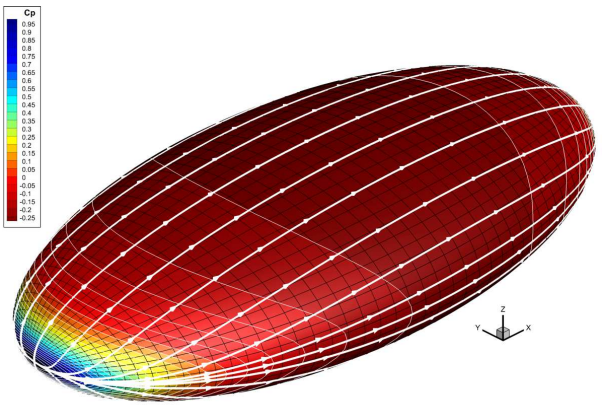

(b) Streamlines and surface pressure distribution.

Figure 3.16: Results of the panel method for a $(4,2,1)$ semi-axes ellipsoid for onset flow in $x$-direction, i.e. $\vec{u}_{\infty}=(1,0,0)^{T}$.

Figure 3.16 shows a result of the panel method for a $64 \times 64$ panel surface grid and a unit onset flow along the longest ellipsoid axis. A corresponding picture for the exact solution can be found in Figure G.2.

\section{Velocity Potential}

The perturbation potential at the collocation point of each of the panels is obtained using a direct solver for the system of equations. The computed perturbation potential at the collocation points is compared to the (linear) analytical solution at the points projected in panel normal direction at the exact ellipsoid surface (see Appendix G.1).

We define the dimensionless $\mathrm{L}_{2}$-norm of the error in the perturbation po- 
tential as

$$
\epsilon_{\varphi}=\frac{\mathrm{L}_{2}\left(\varphi_{\text {exact }}+\mu\right)}{\mathrm{U}_{\text {ref }} \mathrm{L}_{\mathrm{ref}}},
$$

and set the reference length and velocity to $\mathrm{L}_{\text {ref }}=8$ and $\mathrm{U}_{\text {ref }}=1$.

Figure 3.17 shows the error in the velocity potential as a function of the characteristic panel length $h$ defined in equation (3.32). The error is of $\mathcal{O}\left(h^{2}\right)$ in the velocity potential for this paneling. The largest errors occur near the poles of the grid, which in this case coincide with the areas of largest surface curvature. In the case of an onset flow in $x$-direction the stagnation points coincide with the poles of the grid, and the largest errors occur elsewhere on the surface. A similar $\mathcal{O}\left(h^{2}\right)$ super convergence behavior

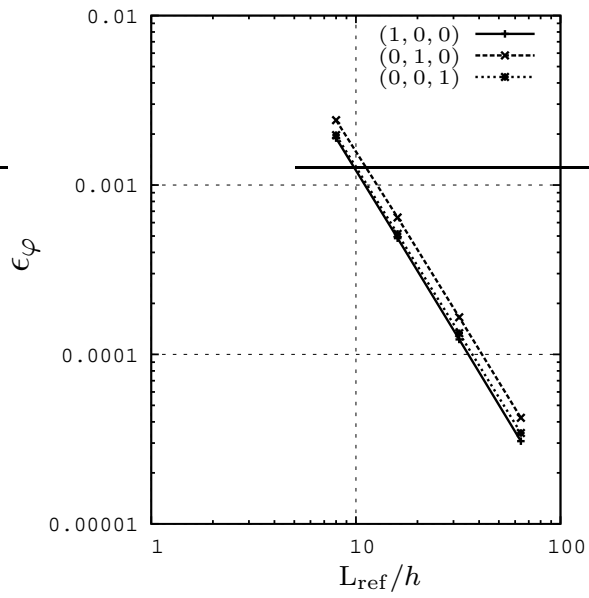

(a) $L_{2}$-norms.

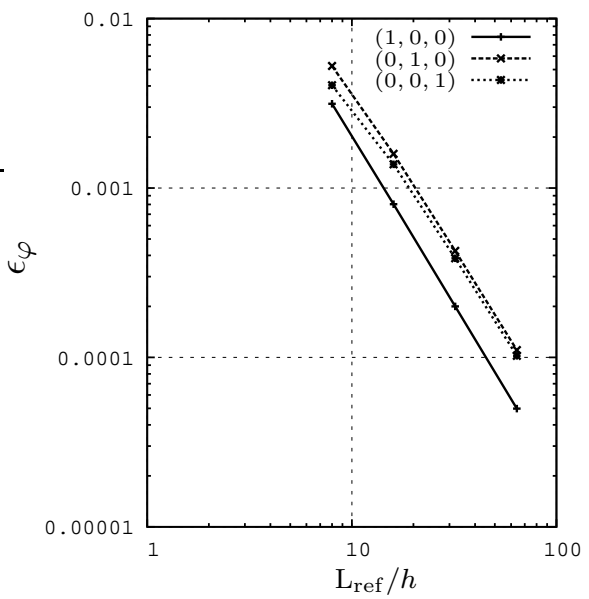

(b) $L_{\infty}$-norms.

Figure 3.17: Norms in the error in perturbation potential as a function of $\mathrm{L}_{\mathrm{ref}} / h$, with $h$ a characteristic panel length, for unit onset flows along the three coordinate axes of a $(4,2,1)$ semi-axes ellipsoid.

was observed in the solid-angle test case in Section 3.5.2. In this case the exact solution is linear in the Cartesian coordinates along the surface of the ellipsoid and the evaluation of the velocity potential in the panel mid point could again be a factor contributing to this occurrence of super convergence.

\section{Surface Pressure Coefficient}

The pressure coefficient is obtained via the Bernoulli equation (2.43) from the velocity computed at the surface. The onset velocity is the reference velocity used to obtain the non-dimensional coefficients. 
Figure 3.18 shows the $\mathrm{L}_{2^{-}}$and $\mathrm{L}_{\infty}$ error norms in the pressure coefficient for onset flows along all three coordinate axes. The behavior of the error in the pressure coefficient is of $\mathcal{O}\left(h^{2}\right)$, but is less regular than the error in the velocity potential. The largest errors occur at points near the poles of the grid for an onset flow in $z$-direction.

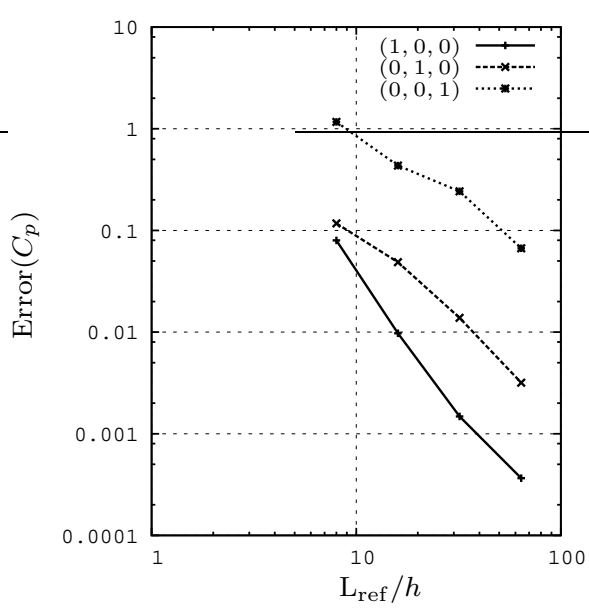

(a) $L_{2}$-norms.

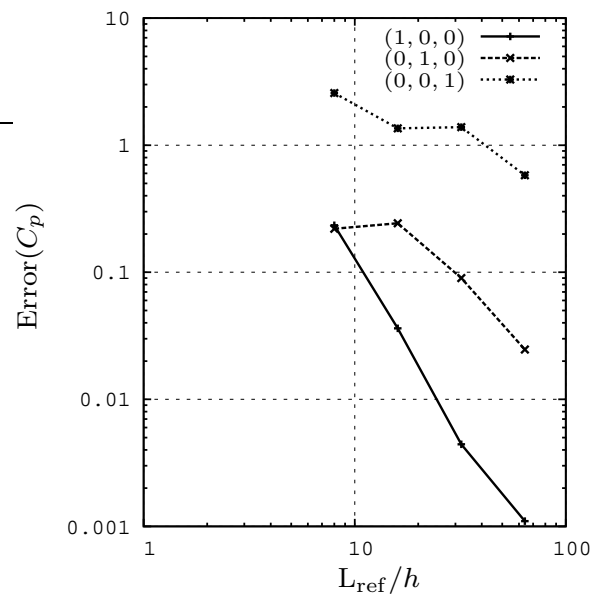

(b) $L_{\infty}$-norms.

Figure 3.18: Norms in the error in pressure coefficient as a function of $\mathrm{L}_{\mathrm{ref}} / h$, with $h$ a characteristic panel length, for unit onset flows along the three coordinate axes of a $(4,2,1)$ semi-axes ellipsoid.

In Figure 3.19, the distribution of the error in the pressure coefficient $\Delta C_{p}=$ $C_{p_{\text {panel }}}-C_{p_{\text {exact }}}$ is plotted for a $64 \times 64$ panel discretization and an onset flow in $z$-direction, the 'worst case' of the three flow conditions. The highest levels of the error occur at the poles of the grid but rapidly decrease away from these points. 


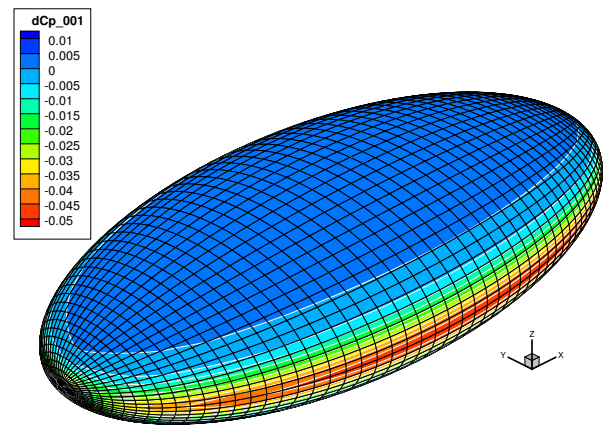

(a) Pressure coefficient error.

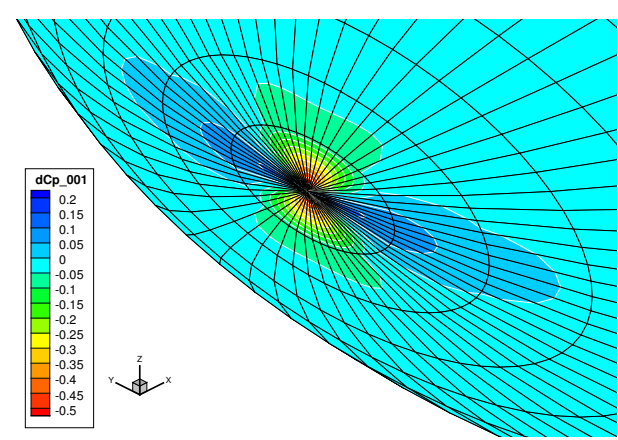

(b) Closeup of the pole region.

Figure 3.19: Distributions of the error in pressure coefficient for a $64 \times 64$ discretization of a $(4,2,1)$ semi-axes ellipsoid in an onset flow in $z$-direction. The largest errors appear at points near the poles of the grid.

\subsubsection{Rotating Ellipsoid in Fluid at Rest}

Exact solutions are available for the incompressible, inviscid flow about a tri-axial ellipsoid in a fluid at rest, rotating at constant angular velocity around an arbitrary axis through its center (see Appendix G.3).

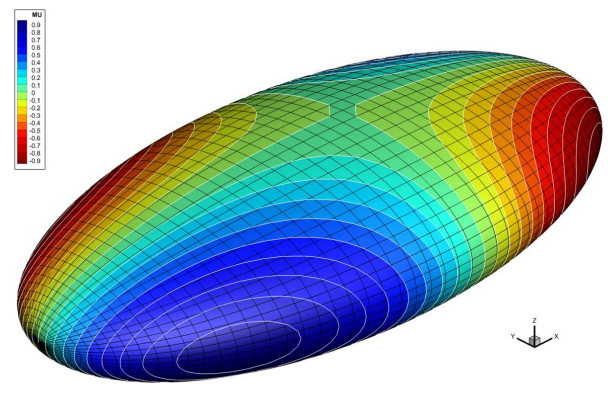

(a) Dipole strength.

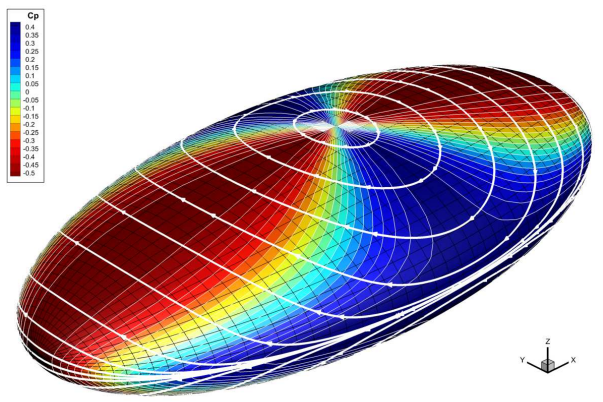

(b) Streamlines and pressure distribution.

Figure 3.20: Results of the panel method for a $(4,2,1)$ semi-axes ellipsoid rotating around the $z$-axis at 1 radian per second angular velocity in a fluid at rest.

The verification tests are performed for an ellipsoid with semi-axes $(4,2,1)$ rotating around each of its three main axes. The surface discretizations are identical to those of the preceding section for this ellipsoid in a uniform parallel flow. In the simulations the geometry is rotated by $1^{\circ}$ each time step as described in Section 3.2.1 until the ellipsoid is in its original position 
again. The results at the last time step are then compared to the exact solution. The time step size $\Delta t$ is such that a rotational velocity of 1 radian per second results, that is, $\Delta t=2 \pi / 360 \mathrm{~s}$.

Figure 3.20 shows a result of the panel method for a $64 \times 64$ panel surface grid and rotation of the ellipsoid at 1 radian per second around the $z$-axis. A corresponding picture for the exact solution can be found in Figure G.3.

\section{Velocity Potential}

Like the case for the ellipsoid in uniform onset flow, the perturbation potential at the collocation points is compared to the (bi-linear) analytical solution at the points projected in panel normal direction located on the exact ellipsoid surface.

We define the dimensionless relative $\mathrm{L}_{2}$-norm of the error in the perturbation potential as in Equation (3.34) and set $\mathrm{L}_{\mathrm{ref}}=8$ and $\mathrm{U}_{\mathrm{ref}}=|\vec{\Omega}| \mathrm{L}_{\mathrm{ref}} / 2=$ 4 , in which we used an angular velocity $|\vec{\Omega}|$ of 1 radian per second.

The error in the velocity potential is shown in Figure 3.21 as a function of the characteristic panel length $h$. The accuracy of the panel method for this test case is $\mathcal{O}\left(h^{2}\right)$. A similar behavior was observed in the test case for the ellipsoid in uniform onset flow and in Section 3.5.2. In this test case the exact solution is bi-linear in the Cartesian coordinates along the surface of the ellipsoid and the evaluation of the velocity potential in the panel mid point could again be a factor contributing to this occurrence of super convergence.

\section{Surface Pressure Coefficient}

The surface pressure coefficient is obtained via the Bernoulli equation (2.43) from the velocity at the surface and the material derivative of the perturbation velocity potential. However, in this case a reference velocity constructed from the (zero) onset velocity and the local surface velocity would give a zero reference velocity at points on the rotation axis and lead to an amplification of the error in pressure coefficient near these points. Therefore, a constant reference velocity of $\mathrm{U}_{\text {ref }}=|\vec{\Omega}| \mathrm{L}_{\text {ref }} / 2=4$ is used to obtain the non-dimensional pressure coefficients and determine the surface distribution of the error.

Figure 3.22 shows the $\mathrm{L}_{2}$ - and $\mathrm{L}_{\infty}$ norms for the error in the pressure coefficient for rotation along each of the three coordinate axes. The behavior of the error in pressure coefficient is less regular than the error in the velocity 


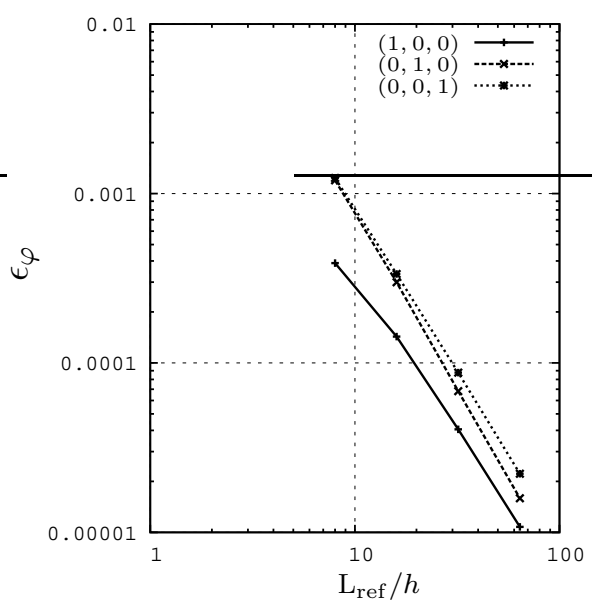

(a) $L_{2}$-norms.

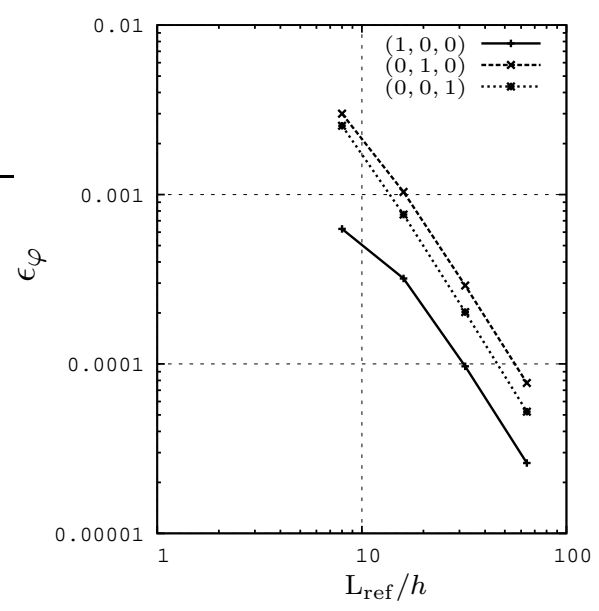

(b) $L_{\infty}$-norms.

Figure 3.21: Norms of the error in perturbation potential as a function of $\mathrm{L}_{\mathrm{ref}} / h$, with $h$ a characteristic panel length, for rotation along the three coordinate axes of a $(4,2,1)$ semi-axes ellipsoid.

potential.

For an ellipsoid discretized with a surface grid of $64 \times 64$ panels the distribution of the error in the pressure coefficient $\Delta C_{p}$ is plotted in Figure 3.23. The ellipsoid rotates with unit angular velocity around the $y$-axis, the "worst case' of the three conditions. The highest error level occurs around the poles of the grid, but the error level rapidly decreases away from these points. 


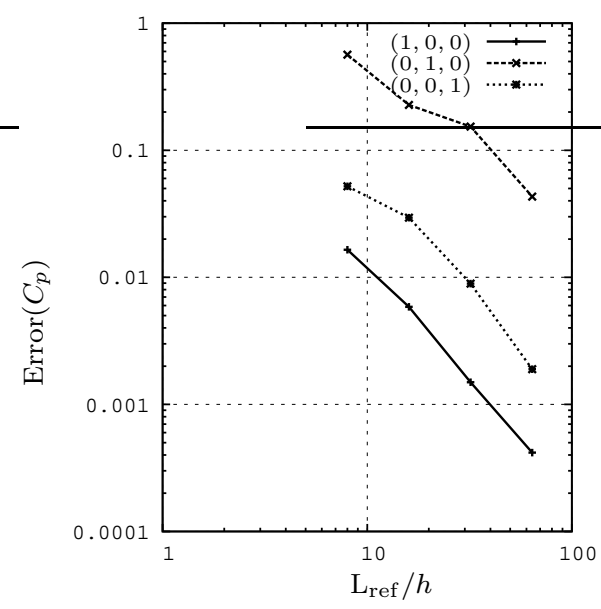

(a) $L_{2}$-norms.

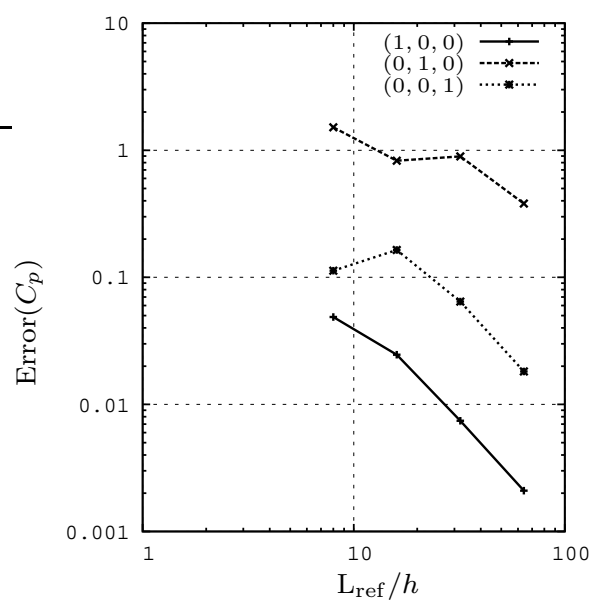

(b) $L_{\infty}$-norms.

Figure 3.22: Norms of the error in pressure coefficient as a function of $\mathrm{L}_{\mathrm{ref}} / h$, where $h$ is a characteristic panel length, for rotation along the three coordinate axes of a $(4,2,1)$ semi-axes ellipsoid.

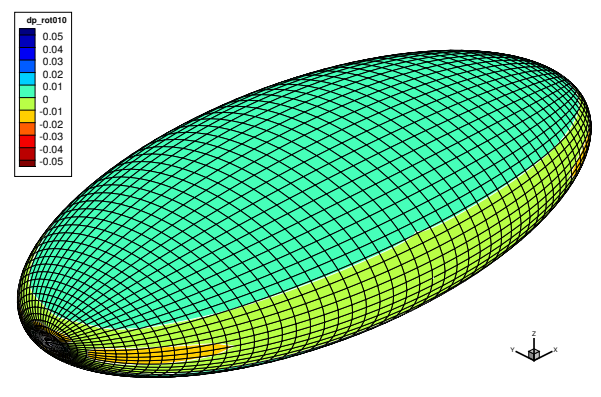

(a) Pressure coefficient error.

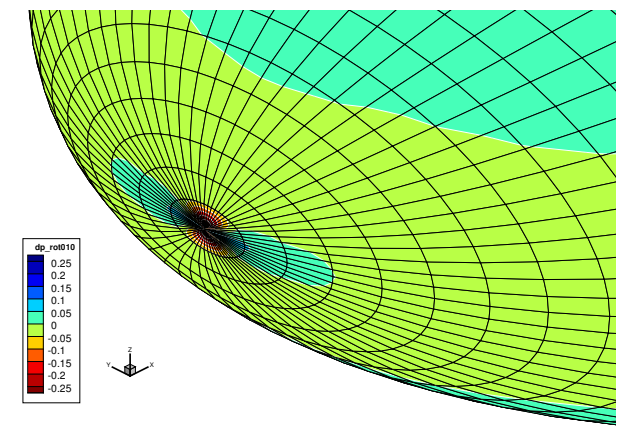

(b) Closeup of the pole region.

Figure 3.23: Surface distribution of the error in the pressure coefficient for a $64 \times 64$ panel discretization of the ellipsoid, rotating around the $y$-axis in a fluid at rest. The largest errors appear in the poles of the grid. 


\subsubsection{Elliptic Wing with von Kármán-Trefftz Airfoil}

For three dimensional lifting bodies there is no analytical solution available. However, it is possible to determine the convergence behavior of the simulation method with the help of the exact solution for a 2D von Kármán-Trefftz (KT) airfoil.

We construct a $21.89 \%$ thick KT airfoil with $4.05 \%$ camber and a trailing edge opening angle of $15^{\circ}$ by a conformal transformation of a circle with center $(-0.15,0.10)$. The downstream stagnation point at the circle at $(1.0,0.0)$ will map into the trailing edge after the transformation. The angleof-attack for zero lift is for the resulting airfoil $\alpha_{0} \approx-4.97^{\circ}$. Figure 3.24 shows the resulting $2 \mathrm{D}$ airfoil geometry.

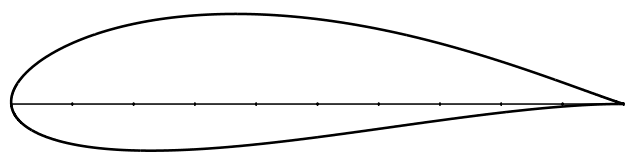

Figure 3.24: Von Kármán-Trefftz airfoil geometry.

The KT airfoil is discretized and represented by 32, 64, 128, 256, and 512 panels. These discretized airfoils are then used as cross sections in a wing with elliptic planform and straight $c / 4$-line. A wing with root chord $c_{r}=1.0$ and wing span $b=200.0$ is selected. This results in a wing with aspect ratio $\lambda=b^{2} / S=4 b /\left(\pi c_{r}\right) \approx 254.65$ which ensures an almost $2 \mathrm{D}$ flow field in case of a uniform onset flow in chordwise direction. The effect of the finite wing span on the lift curve is in the test accounted for by an increase in angle of attack:

$$
\sin \left(\alpha_{3 \mathrm{D}}-\alpha_{0}\right)=\frac{\lambda+2}{\lambda} \sin \left(\alpha_{2 \mathrm{D}}-\alpha_{0}\right)
$$

In the current test we set $\alpha_{2 \mathrm{D}}=5^{\circ}$ and find for the corresponding 3D angle-of-attack $\alpha_{3 \mathrm{D}} \approx 5.0791^{\circ}$. The discretization of the wing is further determined by 17 sections that are distributed more densily towards the wing tips with a cosine function in spanwise direction. At the wing trailing edge a wake is attached with a length in downstream direction of 5 times the wing span.

The section in the wing center is used for the comparison with the solution for the 2D KT airfoil. Figure 3.25 compares the exact (2D) pressure distribution in chordwise direction with the computed results for the $3 \mathrm{D}$ high aspect ratio wing at corrected angle-of-attack. The computed result converges towards the exact result. 


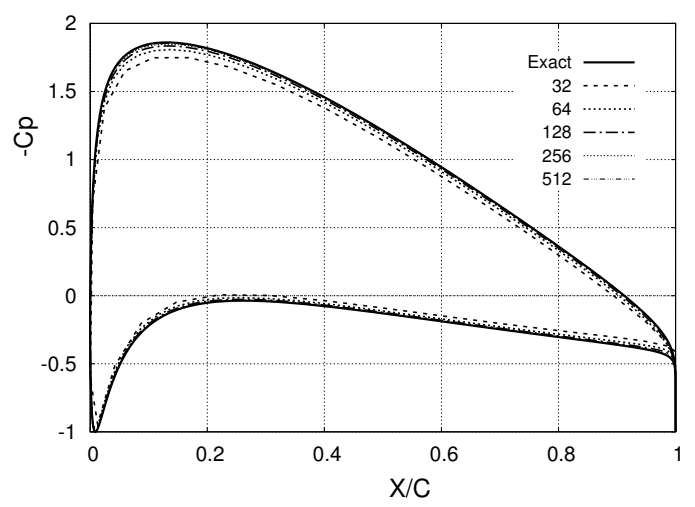

(a) Pressure distribution.

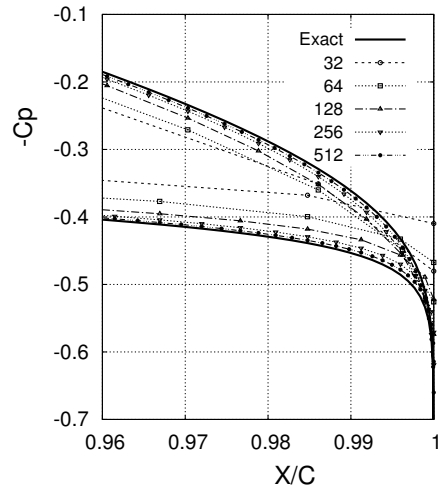

(b) Closeup.

Figure 3.25: Exact (2D) and computed (3D) surface pressure distributions for a wing with a von Kármán-Trefftz airfoil section discretized for a range of chordwise number of panels.

Figure 3.26 shows the norms of the error in the pressure coefficient as function of $\mathrm{L}_{\text {ref }} / h$. The reference length is the root chord $c_{r}$ and the characteristic panel size $h$ is the root chord divided by half the number of panels in chordwise direction. The $\mathrm{L}_{2}$-norm of the error in the pressure coefficient distribution is only slowly decreasing with decreasing panel size and is $\mathcal{O}\left(h^{0.70}\right)$. The reduction in $\mathrm{L}_{\infty}$-norm is much slower $\mathcal{O}\left(h^{0.19}\right)$ and can be attributed to the difference in pressure distribution at the trailing edge where the flow velocity at the surface rapidly decelerates towards the trailing edge and the exact pressure coefficient takes the stagnation value of 1 .

The reduced convergence rate for the lifting wing makes it plausible that the $\mathcal{O}\left(h^{2}\right)$ convergence observed in the test cases for the solid angle (Section 3.5.2), the ellipsoid in uniform onset flow (Section 3.5.3), and the rotating ellipsoid in flow at rest (Section 3.5.4), was due to the constant, linear, and bi-linear character of these exact solutions. 


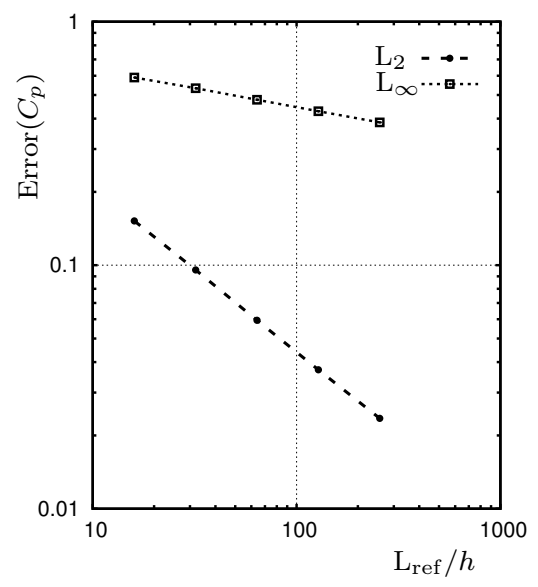

Figure 3.26: Convergence of the computed surface pressure distribution towards the exact solution for a von Kármán-Trefftz airfoil. 


\section{Fast Cluster Multilevel Algorithm}

\subsection{Introduction}

An inherent property of the integral equations discussed in Chapter 2 is that the solution at each location in the domain requires the evaluation of integrals over the entire surface. In the discretization as a panel method (see Chapter 3), the effort to obtain a solution in $N$ points from the integrals over $N$ panels on the surface of the geometry by a direct, conventional approach grows quadratically with the problem size $N$.

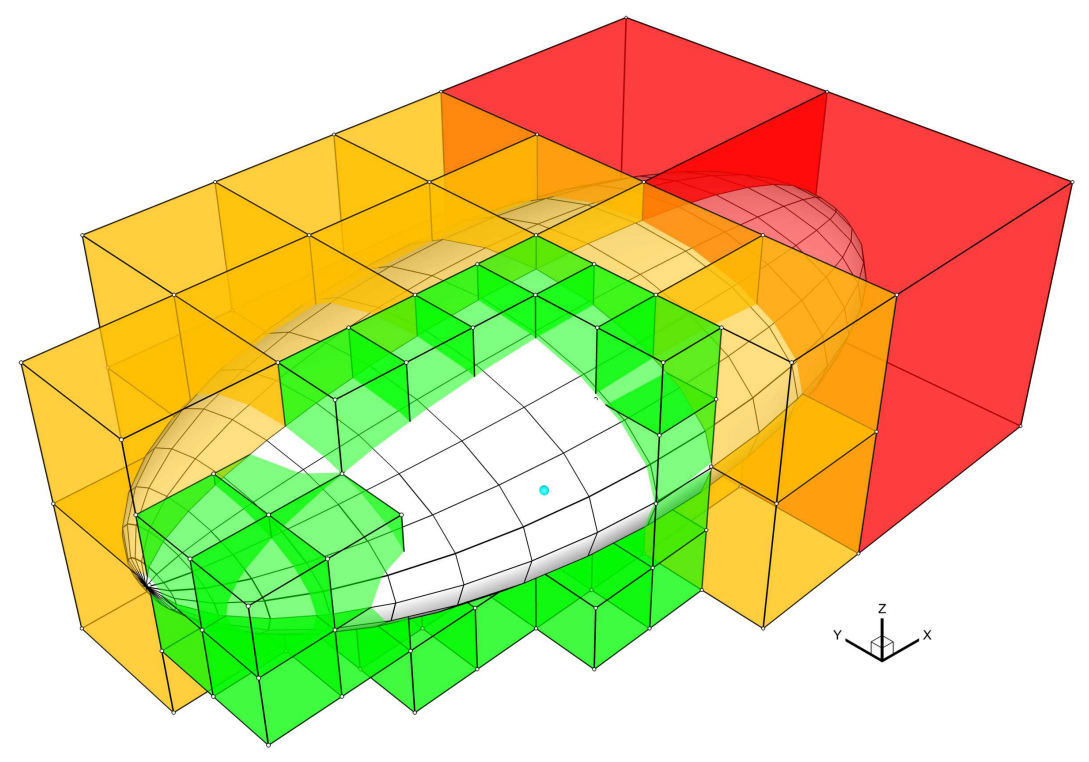

Figure 4.1: Hierarchy of boxes around an evaluation point on a surface.

We apply the panel method to the simulation of unsteady three-dimensional incompressible inviscid flow around wind turbine rotor blades. The wakes that emanate from the rotor blades increase the problem size every time step and the problem easily reaches hundreds of thousands of panels for a single rotor. Moreover, each new time step the geometry is altered and a new evaluation has to be performed.

In a wind turbine engineering environment short problem turnaround times are a prerequisite in the iterative computational rotor blade design pro- 
cess and in the wind turbine structural dynamics analysis phase. Problem turnaround times consists of the preparation time needed to setup a simulation plus the actual simulation run time. Therefore, a conventional $\mathcal{O}\left(N^{2}\right)$ approach quickly becomes infeasible as Figure 4.2 illustrates for a single time step in a relevant 3D wake roll-up simulation. The development of advanced multilevel algorithms provides a very significant alleviation of this computational burden. In our work it is proven that a practical solution can be developed that reduces this burden to $\mathcal{O}(N)$ work. The significance of the multilevel method developed in this work is already showcased in the same figure and its advantage for large problem sizes is obvious.

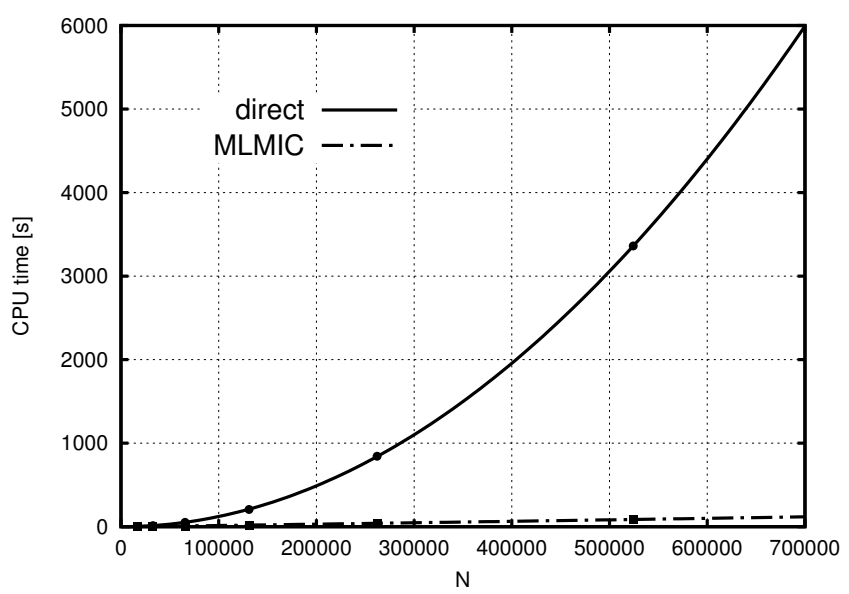

Figure 4.2: Work grows quadratically with problem size $N$ for a conventional panel method implementation. The new MLMIC method reduces the work in each time step for a $3 D$ wake deformation problem considerably.

The algorithm builds upon the multilevel multi-integration concept of Brandt and Lubrecht [23], Brandt [21], Venner and Lubrecht [111]. New concepts are introduced so that the method is also practically applicable to highly irregular surfaces as appearing in the (panel method) wakes of wind turbine rotor blades. We will refer to the new approach as the MLMIC (Multi-Level Multi-Integration Cluster) scheme.

- The concepts and key ideas are explained that will enable us to reduce the work from $\mathcal{O}\left(N^{2}\right)$ to a problem of $\mathcal{O}\left(N \ln ^{d}(N)\right)$ work in $d$-dimensional space. Moreover, the algorithm developed can also handle highly irregular surfaces (Section 4.2).

- The fast multilevel approach for the evaluation of the surface integrals 
is first explained using a model point discretization of the geometry in 1-dimensional space and a smooth, non-singular integral kernel function (Section 4.2.1). It is shown that in this case the work for the evaluation of the integrals is reduced from $\mathcal{O}\left(N^{2}\right)$ to $\mathcal{O}(N)$.

- For singular, asymptotically smooth kernels we define a near field in the domain. We construct the new MLMIC fast multilevel multiintegration scheme centered around the concept of clusters of evaluation points and source points (Section 4.2.2).

The computational effort of the evaluation of the integrals is shown to be of $\mathcal{O}\left(N \ln ^{d}(N)\right)$ for fixed geometries in d-dimensional space.

For the case of developing wakes in wind turbine flows where the geometry grows in size at the same rate as the increase in problem size, the computational effort is reduced to $\mathcal{O}(N)$.

- We show the extension of the MLMIC method with point discretization in one dimension to higher dimensional space (Section 4.3.1) and introduce a hierarchy of volumetric boxes as shown in Figure 4.1.

- The extension of point discretizations to higher order surface (panel) integrals in three-dimensional space is discussed (Section 4.3.2).

- We describe how more elaborate vector (component) kernel functions can be treated and how the gradient of the solution is determined (Section 4.3.3).

- The implementation of the fast MLMIC method for 3D problems is verified and its performance in terms of accuracy and work in a panel method model problem are demonstrated (Section 4.5).

- The relation between the new MLMIC method and other multilevel schemes is discussed (Section 4.6).

\subsection{Multi-Level Multi-Integration Cluster Scheme}

Before describing the basic concepts of the MLMIC method first some remarks on the wording in the rest of this chapter.

The term source is used in its general meaning and refers to any kernel function ${ }^{1}$. Receivers denote the locations where the evaluation of an integral

\footnotetext{
${ }^{1}$ For panel methods the term source is usually reserved for the velocity potential integral (2.11) involving kernel $1 / r$ and its gradient, and the term dipole or doublet is linked to integral (2.10) involving kernel $\bar{n} \cdot \vec{r} / r^{3}$ and its gradient.
} 
transform is needed. The term node is used exclusively for vertices in the (background) grid boxes and for all other situations the word point is used. We use the term box for a rectangular bounded domain, and a set of points in such a bounded domain is called a cluster. A box containing a cluster is said to be active. Furthermore, we will use lowercase and upper case letters for fine and coarse grid variables respectively. Identifier $x$ and subscript $i$ are used for receiver quantities, and identifier $y$ and subscript $j$ for source quantities. Discrete values are identified with a superscript $h$. For the $d$-dimensional formulation subscripts $1, \ldots, d$ will be used.

The canonical form of the integral transform in $d$-dimensional space $\Omega \in \mathbb{R}^{d}$ is

$$
\phi(x)=\int K(x, y) \sigma(y) d y,
$$

which expresses the value of $\phi(x)$ in receiver point $x$ as the integral of the product of a source function $\sigma(y)$ in $y$ and a kernel function $K$ that depends both on $x$ and $y$, where possibly the domains of $x$ and $y$ overlap. Often both receiver and source domains $x$ and $y$ are lower dimensional manifolds $\partial \Omega \in \mathbb{R}^{d-1}$ but this is not a requirement. In linear potential theory the kernel function $K(x, y)$ is a Green's function that only depends on the relative position of source and receiver points $K(x, y)=K(|x-y|)$. However, this property is not required for the multilevel algorithm to be applicable.

The continuous integral transform (4.1) is discretized (using the panel method) and evaluated in receiver points $x_{i}^{h}$ as

$$
\phi^{h}\left(x_{i}^{h}\right)=\phi_{i}^{h}=\int K\left(x_{i}^{h}, y\right) \tilde{\sigma}^{h}(y) d y=\sum_{j} K_{i, j}^{h h} \sigma_{j}^{h},
$$

where $\tilde{\sigma}^{h}(y)$ is a piecewise polynomial representation of the source distribution and the degrees of freedom $\sigma_{j}^{h}$ and $K_{i, j}^{h h}$ defined such that equation (4.2) holds. In the panel method in $\mathbb{R}^{d}$ the domain of integration is most often a surface in $\mathbb{R}^{d-1}$ discretized using a distribution of non-overlapping panels. In case of a piecewise constant source distribution, each degree of freedom $\sigma_{j}^{h}$ used to describe the source distribution is associated with exactly one panel. For clarity it is assumed first that the source distribution is concentrated in points $y_{j}^{h}$ with strength $\sigma_{j}^{h}$ in one-dimensional space, that is, $\tilde{\sigma}^{h}(y)=\sigma_{j}^{h} \delta\left(y-y_{j}^{h}\right)$. In this case $K_{i, j}^{h h}=K\left(x_{i}^{h}, y_{j}^{h}\right)$. We also assume that we have a scalar kernel function. In Section 4.3 we will reintroduce distributed sources, higher dimensional space, and deal with more elaborate kernel functions.

In the MLMI algorithm by Brandt and Lubrecht [23], Brandt [21], and Venner and Lubrecht [111] a fast evaluation is obtained by the combination 
of geometric coarsening and interpolation. The boundary $\partial \Omega$ is represented by a hierarchy of coarsened grids (levels) that are constructed from the definition of the boundary geometry. For singular kernel functions the algorithm exploits the smoothness of the kernel function for distant receiver and source points on the boundary by replacing the exact values of the kernel by polynomial interpolation from coarse grid values. The influence of sources on receivers at the same grid level are corrected when the receiver and source points are too close and the interpolation of the kernel function becomes inaccurate. A prerequisite for such a scheme is that the underlying geometry and parametrization is sufficiently smooth to warrant the kernel interpolation. Wind turbine rotor wakes however deform strongly under self-influence and the resulting manifolds are highly irregular even under normal flow conditions.

The key idea in this thesis is that the kernel $K(x, y)$ is generally a well defined function not only on boundary $\partial \Omega \in \mathbb{R}^{d-1}$ but throughout the domain $\Omega \in \mathbb{R}^{d}$. This observation is used to remove the smoothness constraint on boundary $\partial \Omega$ when $y \in \partial \Omega$ through the introduction of (background) grids in $\Omega$ where grid smoothness can be enforced and $y \in \Omega$. This will make it possible to apply the scheme even in cases with highly distorted surfaces such as deformed wind turbine wake vortex sheets. Obviously this new flexibility comes at the cost of the introduction of an extra space dimension. Volume grids were also discussed by Brandt [21],[22] in the context of many-body interactions.

When smoothness of the boundary and its parametrization can be guaranteed the extra space dimension is not necessary. See for example the paper by Dölz, Harbrecht and Peters [28].

\subsubsection{Smooth Kernels}

We start the explanation of the MLMIC method on the basis of a simple one-dimensional formulation of the discretized integral transform (4.2) exhibiting a smooth, non-singular kernel function $K(x, y)$ with bounded higher order derivatives. In the general case we divide space into $c$ boxes, an approach that was already hinted at by Brandt and Lubrecht [23]. In this section however we will mostly deal with just one cluster of receiver points that fits in a box, and one cluster of source points that fits in a box of its own. This is illustrated in Figure 4.3 where for clarity the points and grids for the two source and receiver boxes are pulled apart. The two boxes are independent, they can overlap or be completely disjoint.

First, we introduce grid nodes $I$ in $X_{I}^{H}$ for the receiver box and grid nodes $J$ 


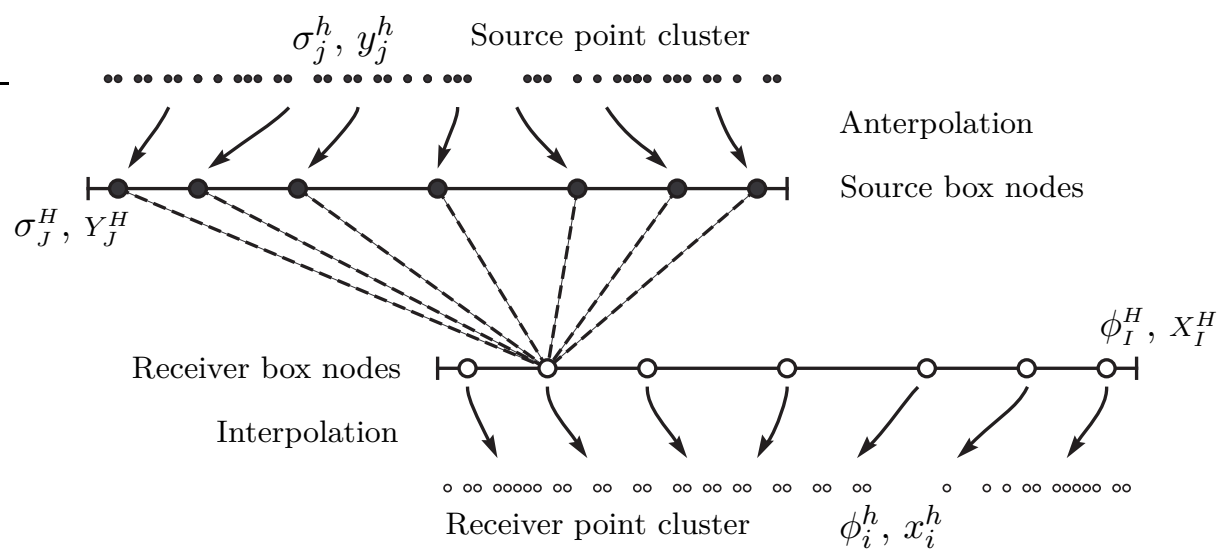

Figure 4.3: Concepts in the MLMIC algorithm for smooth kernel integrals using points and grid boxes in $1 D$ space. For clarity, the points and grids for the source and receiver boxes are pulled apart.

in $Y_{J}^{H}$ for the box on the source side. In addition, we introduce $\phi_{I}^{H}$ and $\sigma_{J}^{H}$ for the integral values in the receiver box nodes and the source strengths in the source box nodes respectively.

The key ingredient in fast multilevel evaluation schemes is an approximate representation of the kernel function $K(x, y)$. In the panel clustering scheme by Hackbusch and Nowak [49] the kernel function is approximated by a Taylor expansion in the source coordinates, and in the Fast Multipole Method by Greengard and Rokhlin [42],[41] spherical harmonics expansions are used. In both these approaches higher order derivatives of the kernel function are required. In the work of Brandt and Lubrecht [23] the kernel function is approximated by an interpolating polynomial requiring only kernel values. This is the approach taken in the MLMIC method.

For convenience let us write the discretized canonical integral transform here again

$$
\phi_{i}^{h}=\sum_{j} K_{i, j}^{h h} \sigma_{j}^{h}
$$

and introduce the approximation for kernel values $K_{i, j}^{h h}$ by interpolating the kernel function through $p$ receiver and source grid nodes $X_{I}$ and $Y_{J}$. For the interpolating polynomials on the receiver and source sides we use the shorthand notations $L^{I}(x)$ and $L^{J}(y)$ where summation over the $p$ indices $I$ and $J$ is implied. We can now write

$$
K_{i, j}^{h h}=\tilde{K}_{i, j}^{h h}+\mathcal{O}(\epsilon)
$$


where

$$
\tilde{K}_{i, j}^{h h}=\sum_{I} \sum_{J} K_{I, J}^{H H} L^{I}\left(x_{i}^{h}\right) L^{J}\left(y_{j}^{h}\right)
$$

and

$$
K_{I, J}^{H H}=K\left(X_{I}^{H}, Y_{J}^{H}\right)
$$

We will subdivide the domain into multiple boxes of the same size and use Lagrange interpolation through $p$ background grid nodes in each of the active boxes that encompass receiver and source point clusters. The error we make by this approximation is of $\mathcal{O}\left(K^{(p)} h^{p}\right)$, with $h$ the size of the box. The only requirement for the receiver and source background grids is that the kernel function can be represented accurately by polynomial interpolation on these grids. In our implementation we will use a Chebyshev node distribution of the first kind where the nodes in each grid box are distributed more densely towards the boundaries of the box. This results in a more evenly distributed interpolation error. An accessible description of the barycentric form of Lagrange interpolation and the advantages of using Chebyshev nodes is discussed by Berrut and Trefethen [9]; see also Appendix F.

Substitution of (4.5) into (4.3) gives

$$
\phi_{i}^{h} \approx \sum_{j} \sum_{I} \sum_{J} K_{I, J}^{H H} L^{I}\left(x_{i}^{h}\right) L^{J}\left(y_{j}^{h}\right) \sigma_{j}^{h},
$$

which can be reordered without any assumptions into

$$
\phi_{i}^{h} \approx \sum_{I} L^{I}\left(x_{i}^{h}\right)\left(\sum_{J} K_{I, J}^{H H}\left(\sum_{j} L^{J}\left(y_{j}^{h}\right) \sigma_{j}^{h}\right)\right) .
$$

In equation (4.8) the three components in the MLMIC method for smooth kernels can be distinguished going inward-out of the parentheses. With the help of Figure 4.3, and introducing some new nomenclature, the three steps are:

\section{Anterpolation}

The source strengths $\sigma_{j}^{h}$ in points $y_{j}^{h}$ are effectively transferred to "pseudo-sources" $\sigma_{J}^{H}$ located in the encompassing box grid nodes $Y_{J}^{H}$ by

$$
\sigma_{J}^{H}=\sum_{j} L^{J}\left(y_{j}^{h}\right) \sigma_{j}^{h} .
$$

This step is the adjoint (or transpose) of interpolation in $Y_{J}^{H}$, hence its name (see Brandt [21]). 


\section{Coarse grid summation}

The kernel values $K_{I, J}^{H H}$ representing the influence of source box nodes on receiver box grid nodes are used to perform a matrix-vector multiplication with the vector of "pseudo-sources" $\sigma_{J}^{H}$ in source nodes $Y_{J}^{H}$ to give the receiver values $\phi_{I}^{H}$ in the receiver box grid nodes $X_{I}^{H}$ through

$$
\phi_{I}^{H}=\sum_{J} K_{I, J}^{H H} \sigma_{J}^{H}
$$

\section{Interpolation}

The receiver values $\phi_{i}^{h}$ in points $x_{i}^{h}$ are obtained by interpolation of the receiver values $\phi_{I}^{H}$ in the surrounding nodes $X_{I}^{H}$ of the receiver box grid through

$$
\phi_{i}^{h}=\sum_{I} L^{I}\left(x_{i}^{h}\right) \phi_{I}^{H} .
$$

The coarse grid summation in equation (4.10) has a form identical to the original discrete problem in (4.3). We can therefore apply the MLMIC method recursively and introduce a hierarchy of increasingly larger (coarser) grid boxes. Each set of boxes of the same size constitutes a grid level. Figure 4.4 shows an illustration of such interactions between two box grid levels in the MLMIC method where the smaller (fine) grid box nodes now assume the role of the points described earlier. Note that the interpolation is always done within the coarse receiver box under consideration. Anterpolation distributes information only to the nodes of the encompassing "parent" source box.

For smooth kernels with bounded higher order derivatives the recursive invocation of the scheme does not bring any advantage over performing a single step using only the coarsest background grid. However, for asymptoticallysmooth singular kernels we can fully exploit the recursive character of the scheme, as we will see in Section 4.2.2.

For Lagrange interpolation in one-dimensional space with polynomials of order $p$, the number of nodes in each box is $p$. All grid nodes in a box are involved in the interpolation to any point within that box. Furthermore we assume, without loss of generality, that there are an equal number $N$ of receiver points $x_{i}^{h}$ and source points $y_{j}^{h}$.

For the anterpolation step the amount of work for each source point is $\mathcal{O}(p)$ for one-dimensional problems and $\mathcal{O}\left(p^{d}\right)$ in the $d$-dimensional case. Likewise, the work per receiver point in the interpolation step is $\mathcal{O}\left(p^{d}\right)$ for $d$-dimensional problems. For anterpolation and interpolation between grid levels the amount of work per grid node is $\mathcal{O}(d p)$ for problems in 


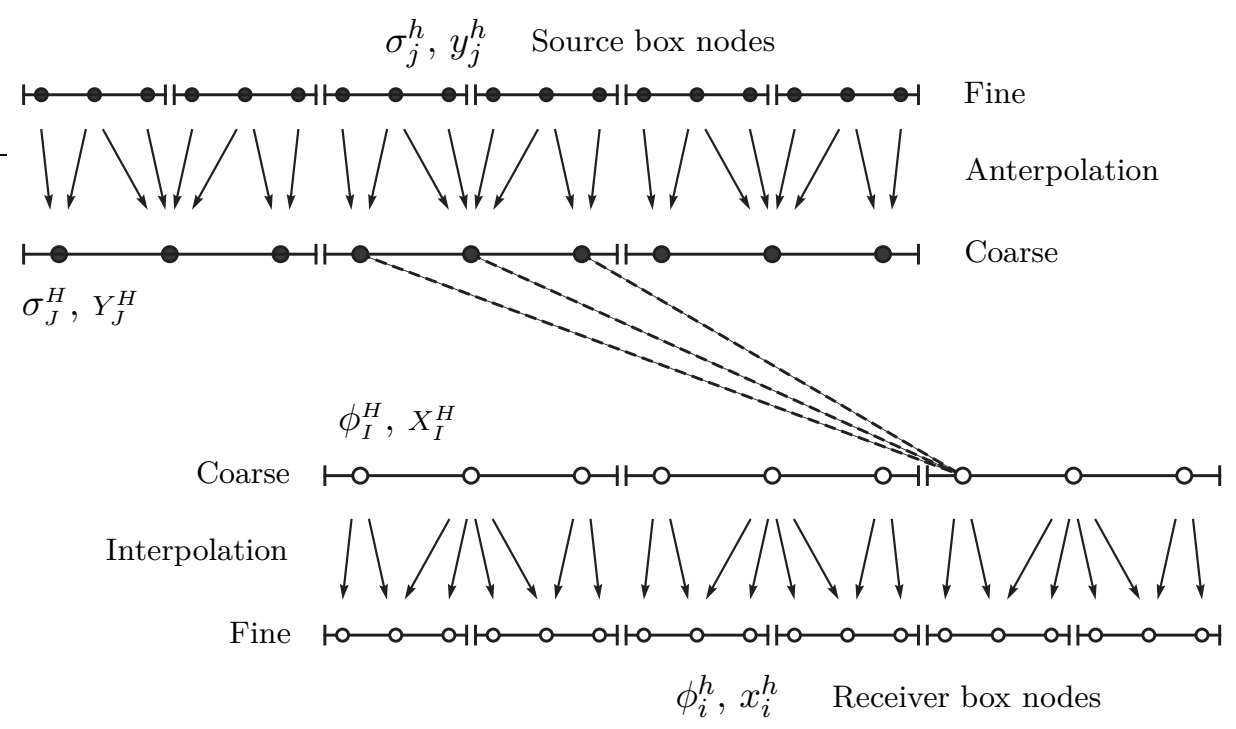

Figure 4.4: Concepts in the MLMIC algorithm for smooth kernel integrals involving boxes on two grid levels in $1 D$ space. For clarity, the source and receiver boxes at the two grid levels are pulled apart.

$d$-dimensional space when those tasks are performed one dimension at a time.

Thus, the work involved in anterpolation and interpolation between all receiver and source points and the boxes at the finest grid level is at most of $\mathcal{O}\left(p^{d} N\right)$. For anterpolation and interpolation in between grid levels the work is of $\mathcal{O}(m d p)$, where $m$ is the number of active nodes on the finer grid level.

If we assume that at the coarsest grid level in 1D space there are $c$ receiver boxes and $c$ source boxes each having $p$ nodes, the amount of work for the coarse grid summation is of $\mathcal{O}\left(c^{2} p^{2}\right)$. For the general $d$-dimensional case the work is of $\mathcal{O}\left(c^{2 d} p^{2 d}\right)$.

The anterpolation and interpolation steps are thus linearly dependent on the number of source and receiver points, assuming the polynomial order $p$ is independent of problem size $N$ for smooth kernel functions. If we now arrange for the $c^{d} p^{d}$ number of background grid nodes at the coarsest level in $d$-dimensional space to be such that at most $c^{2 d} p^{2 d} \propto N$ each of the three steps is linearly dependent on the problem size $N$, and the work for the complete MLMIC method for smooth kernels is $\mathcal{O}(N)$.

The reduction of computational complexity from $\mathcal{O}\left(N^{2}\right)$ to $\mathcal{O}(N)$ is ob- 
tained at the expense of the error made in interpolation which of course should be controlled, i.e. kept within "acceptable" bounds that are determined by the discretization error that is made by the panel method anyway.

For smooth kernels with bounded higher order derivatives on a domain of size $h$ the maximum error is $\left|\epsilon_{p}(x)\right| \in \mathcal{O}\left(h^{p}\right)$ (see Appendix F). For the interpolation order we find thus that $p \in \mathcal{O}(\ln (1 / \epsilon) / \ln (1 / h))$. A reduction in the error level for a given domain size can therefore be achieved with a higher order interpolation polynomial, and for a fixed error level and a decreasing domain size the polynomial order can be lowered.

\subsubsection{Singular Kernels}

For asymptotically smooth, singular kernels like $K(x, y)=|x-y|^{-1}$ the function and its higher order derivatives become unbounded when receiver points $x$ and source points $y$ approach each other. As a result (see Appendix F), a naive replacement of the kernel function by an interpolating polynomial through $p$ nodes in a box of size $h$ will give an unacceptable high error of $\mathcal{O}\left(K^{(p)} h^{p}\right)$ which translates for the above given kernel function to an error of $\mathcal{O}\left(|x-y|^{-(p+1)} h^{p}\right)$. This suggests two measures that can be taken to avoid these problems: decrease the box size $h$ or use the interpolating polynomial only when the kernel function is behaving smoothly and its derivatives are bounded, that is, when receiver points $x$ and source points $y$ are sufficiently distant (according to some criterion yet to be defined). Alternatively one can replace the original kernel with a function with bounded higher order derivatives (kernel softening) and correct for the error a posteriori as for example done by Sandak [98].

To describe the MLMIC method concisely let us introduce the notation $\mathcal{B}^{J}$ for a source box, $\mathcal{B}^{I}$ for a receiver box, and $\{$.$\} for a set. We introduce the$ shorthand $\{i\}$ for the set of receiver points $x_{i}^{h}$ contained in box $\mathcal{B}^{I}$, and $\{I\}$ for the set of receiver box grid nodes $X_{I}^{H}$ in $\mathcal{B}^{I}$. Similarly for the sources: $\{j\}$ denotes the set of source points $y_{j}^{h}$ in box $\mathcal{B}^{J}$, and $\{J\}$ is the set of source box grid nodes $Y_{J}^{H}$ in $\mathcal{B}^{J}$.

The notion of separating space around a receiver box $\mathcal{B}^{I}$ into a near field and a far field is key in the MLMIC method for asymptotically smooth kernels. The MLMIC method is centered around the concept of point clusters contained in boxes, so the natural choice is to distinguish near field and far field based upon the distance between receiver and source box centers:

$$
d=\operatorname{dist}\left(\mathcal{B}^{I}, \mathcal{B}^{J}\right) / h,
$$

where $h$ is the box size and dist() is defined as the maximum of the distances 


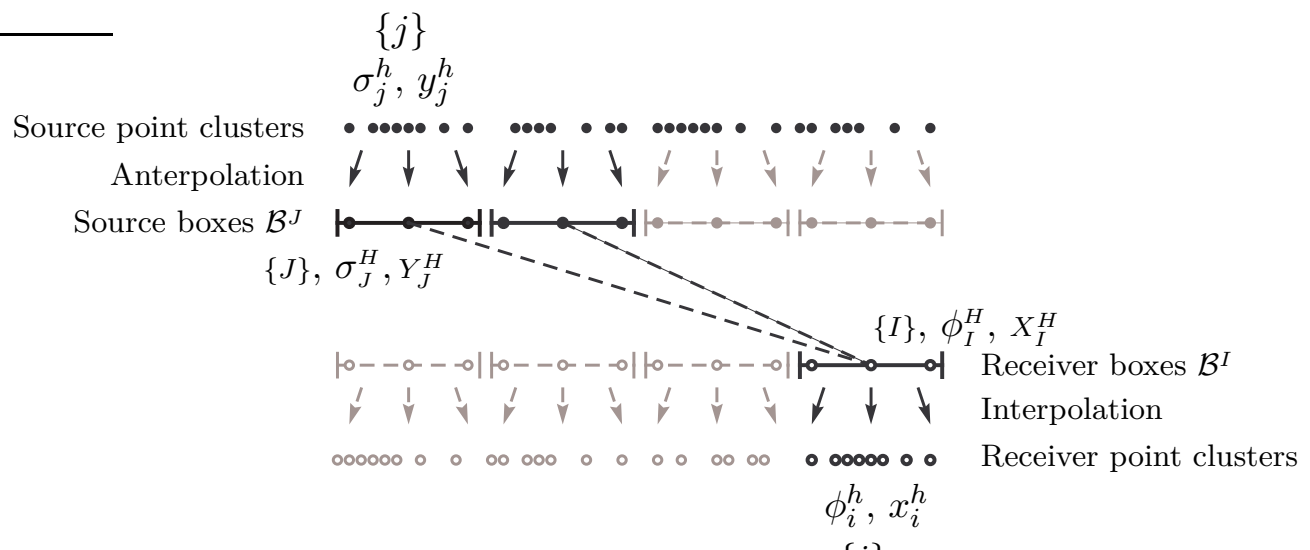

Figure 4.5: Concepts in the MLMIC algorithm for asymptotically smooth, singular kernels using uniform boxes. Source boxes in the near field of a receiver box do not contribute to the result at that level.

in each coordinate direction between the centers. We introduce a near field distance parameter $d_{\mathrm{nf}}$ that defines whether a source box is in the near field of a receiver box or in its far field:

$$
\begin{aligned}
& d \leq d_{\mathrm{nf}} \quad: \quad \text { near field } \\
& d>d_{\mathrm{nf}} \quad: \text { far field. }
\end{aligned}
$$

Thus, a "ring" of $d_{\text {nf }}$ source boxes defines the perimeter of a receiver box near field. For example, in Figure 4.5 where $d_{\mathrm{nf}}=1$ only the immediate neighbors of a receiver box are in its near field.

Let us introduce the notation $\{J\}_{\mathrm{ff}}$ for the source nodes in boxes $\mathcal{B}^{J}$ in the far field of $\mathcal{B}^{I}$, that is, when $\operatorname{dist}\left(\mathcal{B}^{I}, \mathcal{B}^{J}\right) / h>d_{\mathrm{nf}}$. In the same spirit we introduce $\{j\}_{\mathrm{nf}}$ for the clusters of source points in boxes $\mathcal{B}^{J}$ in the near field of $\mathcal{B}^{I}$ where $\operatorname{dist}\left(\mathcal{B}^{I}, \mathcal{B}^{J}\right) / h \leq d_{\mathrm{nf}}$. The resulting MLMIC method for asymptotically smooth kernels is now as follows:

\section{Anterpolation}

For each block $\mathcal{B}^{J}$ compose clusters of source points in $y_{j}^{h} \in \mathcal{B}^{J}$ with strengths $\sigma_{j}^{h}$ and transfer these strengths to "pseudo-sources" $\sigma_{J}^{H}$ located in the source background grid nodes $Y_{J}^{H} \in \mathcal{B}^{J}$ by

$$
\sigma_{J}^{H}=\sum_{\{j\}} L^{J}\left(y_{j}^{h}\right) \sigma_{j}^{h} .
$$




\section{Coarse grid summation}

For each receiver box $\mathcal{B}^{I}$ the kernel values $K_{I, J}^{H H}$ representing the influence of source box $\mathcal{B}^{J}$ on receiver block $\mathcal{B}^{I}$ are used to account for the effects of the "pseudo-sources" $\sigma_{J}^{H}$ in the far field of box $\mathcal{B}^{I}$. The resulting receiver values $\phi_{I}^{H}$ in the receiver box grid nodes $X_{I}^{H} \in \mathcal{B}^{I}$ are obtained through

$$
\phi_{I}^{H}=\sum_{\{J\}_{\mathrm{ff}}} K_{I, J}^{H H} \sigma_{J}^{H} .
$$

By excluding the contributions from the source boxes in the near field the error that would result from interpolation of the kernel function is avoided.

\section{Interpolation}

For each receiver box $\mathcal{B}^{I}$ the receiver values $\phi_{i}^{h}$ in points $x_{i}^{h} \in \mathcal{B}^{I}$ are obtained by interpolation of the receiver values $\phi_{I}^{H}$ at the grid nodes $X_{I}^{H} \in \mathcal{B}^{I}$ of the receiver grid through

$$
\phi_{i}^{h}=\sum_{\{I\}} L^{I}\left(x_{i}^{h}\right) \phi_{I}^{H} .
$$

\section{Cluster near field}

For each receiver box $\mathcal{B}^{I}$ the receiver values $\phi_{i}^{h}$ in points $x_{i}^{h} \in \mathcal{B}^{I}$ are complemented with the contributions from the near field source points with strength $\sigma_{j}^{h}$ by

$$
\phi_{i}^{h}:=\phi_{i}^{h}+\sum_{\{j\}_{\mathrm{nf}}} K_{i, j}^{h h} \sigma_{j}^{h} .
$$

One of the crucial differences between the current scheme and the MLMI scheme by Brandt and Lubrecht [23], Brandt [21], and Venner and Lubrecht [111] is that it avoids the introduction of corrections to the intermediate results on the coarser grids. This is a consequence of the containment of information in the boxes. An additional advantage of the separate final cluster near field contribution in (4.17) is that it avoids computationally expensive removal of self-influences as was encountered by Sandak [98].

The coarse grid summation in equation (4.15) can be carried out recursively using a hierarchy of increasingly larger boxes. The nodes in smaller boxes at the finer grid level take the role of the points described in the algorithm above.

Figure 4.6 depicts the case of two background grid levels where the black bars represent the (far field) source boxes and the white bars the receiver 
boxes that are involved in the construction of the solution in receiver point $x_{i}^{h}$. The source strengths in each box $\mathcal{B}^{J}$ are obtained from anterpolation of the source strengths in its "child" boxes $\mathcal{B}^{j}$. In this example the near field criterion is $d_{\mathrm{nf}}=1$. Each source block contributes to the (partial) result in the receiver block on the same grid level. These partial results are propagated (by interpolation) from each coarser grid level to the nodes of the next finer grid level until the finest grid is reached and the contribution is interpolated in the receiver point $x_{i}^{h}$.

In the final cluster near field step the contributions of all source points $\{j\}_{\mathrm{nf}}$ in the finest grid level near field boxes surrounding $x_{i}^{h}$ are taken into account.

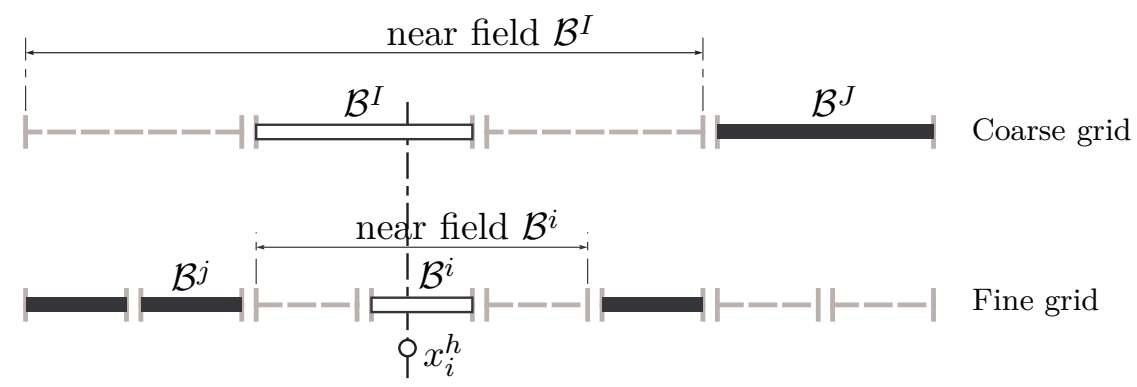

Figure 4.6: Source boxes (black) and receiver boxes (white) on two grid levels involved in the determination of the solution at point $x_{i}^{h}$. The grey boxes are not involved in the partial result at their grid level.

The work involved in the MLMIC method for asymptotically smooth, singular kernels follows the reasoning for smooth kernel functions. We apply the scheme recursively and account for the influence of sources further away with larger boxes on coarser grid levels. A constant polynomial order $p$ and a doubling of the box size with each coarsening is assumed, though other setups are also possible.

From the definition of the near field parameter $d_{\mathrm{nf}}$ in equation (4.13) it follows that a receiver box has $d_{\text {nf }}$ boxes on either side in its near field. Thus, the near field of a receiver box contains $\left(2 d_{\mathrm{nf}}+1\right)^{d}$ source boxes in $d$ dimensional space. The range of active source boxes surrounding a receiver box is determined by the near field of the receiver box's parent; outside that range the coarser grid source boxes are in the far field and take over. This coarser grid near field is also $\left(2 d_{\mathrm{nf}}+1\right)^{d}$ wide and can contain $2^{d}\left(2 d_{\mathrm{nf}}+1\right)^{d}$ "child" source boxes.

The number of active source boxes that contribute to the result in a particu- 
lar receiver box at a specific grid level is therefore at most $\left(2^{d}-1\right)\left(2 d_{\mathrm{nf}}+1\right)^{d}$. In the one-dimensional example in Figure 4.6 , where $d_{\mathrm{nf}}=1$, there are at most 3 sources boxes involved at each grid level. For a three-dimensional problem and $d_{\mathrm{nf}}=1$ this increases to at most 189 active source boxes surrounding each receiver box. Notice that this number grows rapidly with increasing near field distance; for example for $d_{\mathrm{nf}}=2$ we already have (at most) 875 source boxes for each receiver box result on every grid level.

We assume without loss of generality that there are an equal number $N$ of receiver points $x_{i}^{h}$ and source points $y_{j}^{h}$ and assume that all grid levels have the same interpolation order $p$. Each receiver and source box therefore has $p^{d}$ nodes. We further assume that at the finest grid level there are $c^{d}$ receiver and source boxes. We already established that each receiver box on every grid level has a bound maximum number of source boxes that contribute to its result, independent of problem size $N$ and polynomial order $p$. It follows that the work for coarse grid summation for each receiver box on the finest grid level is at most of $\mathcal{O}\left(c^{d} p^{2 d}\right)$. At each coarser grid level the number of boxes is reduced by a factor $2^{d}$ and the total work per grid level consequently reduces with geometric progression. The amount of work per finest grid receiver box and every coarser grid "ancestor" box is therefore still $\mathcal{O}\left(c^{d} p^{2 d}\right)$. When we set the box size on the finest grid level such that the average number of points per box is in the order of the number of nodes in that box, i.e. $N / c^{d} \in \mathcal{O}\left(p^{d}\right)$, the above estimate for the order of work for all coarse grid summations becomes $\mathcal{O}\left(p^{d} N\right)$.

For anterpolation of point sources to the finest grid nodes the amount of work per point is of $\mathcal{O}\left(p^{d}\right)$ giving a total amount of work for all $N$ source points of $\mathcal{O}\left(p^{d} N\right)$. For the anterpolation between the finest grid level and the next coarser level the work per node is of $\mathcal{O}(p)$ if each dimension is handled separately. With $N / c^{d} \in \mathcal{O}\left(p^{d}\right)$ the total numer of nodes on the finest grid is $\mathcal{O}(N)$ and the total work for anterpolation of all nodes on the finest grid level is thus of $\mathcal{O}(p N)$. For coarser grid levels the number of nodes decreases with geometric progression and the total amount of work over all grid levels remains $\mathcal{O}(p N)$. The amount of work involved in the interpolation steps is equal to the work for the equivalent anterpolation steps as follows from the adjoint relationship.

In the final near field cluster contribution the source points in $\left(2 d_{\mathrm{nf}}+1\right)^{d}$ near field boxes are involved and the work for the result in all points in a receiver box is thus of $\mathcal{O}\left(N^{2} / c^{2 d}\right)$. This gives a total work for all $c^{d}$ receiver boxes of $\mathcal{O}\left(N^{2} / c^{d}\right)$. With the number of points per finest grid level box $N / c^{d} \in \mathcal{O}\left(p^{d}\right)$, the total work for the final near field contribution will be of $\mathcal{O}\left(p^{d} N\right)$. This situation occurs when an increase in $N$ is accompanied 
by an equally rapid growing domain and we keep the finest grid box size constant. An example of such a case is of wind turbine rotor wakes that grow and convect further downstream with each new time step.

In the case of an increasing problem size $N$ for a fixed size geometry while keeping the number of finest grid level boxes at $c^{d}$ and consequently the box size constant the total amount of work in the final near field cluster contribution will be $\mathcal{O}\left(N^{2}\right)$.

The order of work in this case can be reduced by decreasing the finest grid box size with increasing problem size. If we want to keep the number of sources per box on the finest grid level independent of problem size, the box size should decrease like $h \in \mathcal{O}\left(1 / N^{1 / d}\right)$ for a uniform distribution of receiver and source points in space. From the interpolation error estimate in equation (F.8) we can deduce for a kernel function like $f(x)=1 / x$ and $d_{\mathrm{nf}}=1$ that $\left|\epsilon_{p}(x)\right| \in \mathcal{O}\left(1 /\left(2^{2 p-1} h\right)\right)$. As a result the polynomial order is $p \in \mathcal{O}(\ln (1 /(\epsilon h)))$ or in terms of problem size $p \in \mathcal{O}(\ln (N / \epsilon))$. Consequently the amount of work in all steps of the MLMIC method for a given accuracy will be of $\mathcal{O}\left(N \ln ^{d}(N)\right)$ for problems in $d$-dimensional space.

\subsection{Higher Dimensions}

This section extends the MLMIC method to two-dimensional domains with point sources, receiver points, and a uniform distribution of source and receiver boxes that cover space. The further extension of the MLMIC method to 3D space is straightforward. The interaction between two consecutive grid levels is discussed. As was recommended in Brandt and Lubrecht [23] and in Venner and Lubrecht [111], the anterpolation and interpolation steps are performed one dimension at a time but in contrast to those references the coarse grid summation step is performed only when all dimensions are coarsened. From now on we omit the superscripts $H$ and $h$ and signal coarse grid variables with capital letters and fine grid variables with lower case letters. Subscripts $1, \ldots, d$ will be used when the direction to which an identifier is associated is unclear from context.

The canonical integral transform (4.1) for the problem in two-dimensional space $\Omega \in \mathbb{R}^{2}$ reads

$$
\phi(\vec{x})=\int K(\vec{x}, \vec{y}) \sigma(\vec{y}) d y .
$$

We will assume again that the source distribution $\sigma_{j}$ is a scalar function and is concentrated in points $\vec{y}_{j}$ and that both source points $\vec{y}_{j}=\left(y_{j_{1}}, y_{j_{2}}\right)^{\mathrm{T}}$ and receiver points $\vec{x}_{i}=\left(x_{i_{1}}, x_{i_{2}}\right)^{\mathrm{T}}$ could be distributed randomly over the 
$2 \mathrm{D}$ domain $^{2}$. The discrete approximation of the integral transform (4.2) is repeated here

$$
\phi_{i}=\sum_{j} K_{i, j} \sigma_{j}
$$

where now index $i$ is used for the set of $2 \mathrm{D}$ receiver points and index $j$ is used for the set of $2 \mathrm{D}$ source points.

Grid nodes are distributed in a (tensor product) matrix ordering. For fine grid receiver and source nodes in 2D space we will use lower case letters $i \equiv\left(i_{1}, i_{2}\right)$ and $j \equiv\left(j_{1}, j_{2}\right)$ respectively where the subscripts 1 and 2 are sometimes omitted and implied by the position of the index instead. For coarse grid nodes upper case indices are used.

\subsubsection{Singular Kernels}

An illustration of the elements in the MLMIC interaction between source point clusters, receiver point clusters, and a single source and receiver box with grid nodes is given in Figure 4.7 where for clarity the points, nodes, and boxes for the source and receiver sides are shifted closer together. Also shown are the interactions between some nodes $\vec{Y}_{J J}$ in the source box with strength $\sigma_{J J}$ and one node in the receiver box which represents part of the coarse grid source contribution to one receiver value $\phi_{I I}$ in grid node $\vec{X}_{I I}$. The arrows in the figure represent the flow of information in the scheme, that is, for anterpolation on the source side the flow is from the source points towards the grid nodes and for interpolation on the receiver side the flow is away from the nodes in the direction of the cluster points.

Given a source box $\mathcal{B}^{J}$ with nodes $\vec{Y}_{J J}$ and a receiver box $\mathcal{B}^{I}$ with nodes $\vec{X}_{I I}$ we can now exploit the smoothness of the kernel $K(\vec{x}, \vec{y})$ by multivariate Lagrange interpolation (see Appendix F) of the discrete function values in the background nodes:

$$
K_{i, j}=\tilde{K}_{i, j}+\mathcal{O}(\epsilon)
$$

where

$$
\tilde{K}_{i, j}=\sum_{I_{1}} \sum_{I_{2}} \sum_{J_{1}} \sum_{J_{2}} K_{I I, J J} L^{I_{1}}\left(x_{i_{1}}\right) L^{I_{2}}\left(x_{i_{2}}\right) L^{J_{1}}\left(y_{j_{1}}\right) L^{J_{2}}\left(y_{j_{2}}\right),
$$

and

$$
K_{I I, J J}=K\left(\vec{X}_{I I}, \vec{Y}_{J J}\right) .
$$

\footnotetext{
${ }^{2} \mathrm{~A}$ slight abuse of notation for the point coordinates will enable us to use it for both point-node interactions and node-node interactions.
} 


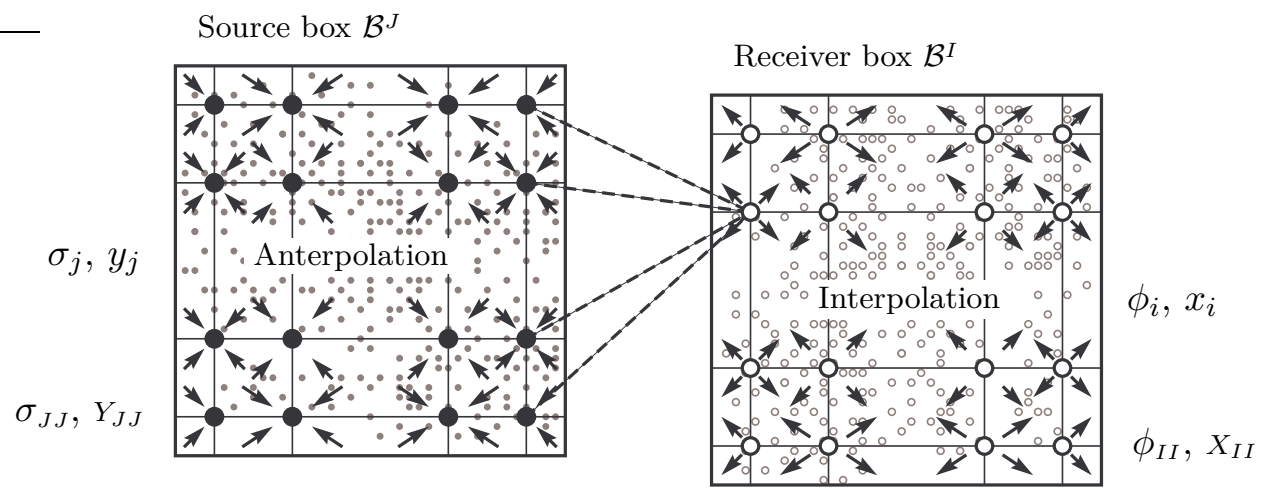

Figure 4.7: Concepts in the MLMIC interaction between source point and receiver point clusters, and between source and receiver box grid nodes in $2 D$ space.

Substitution of the approximation for the kernel function in (4.21) into the original discrete summation in equation (4.19) gives after some reordering

$$
\phi_{i} \approx \sum_{I_{1}} L^{I_{1}}\left(x_{i_{1}}\right) \sum_{I_{2}} L^{I_{2}}\left(x_{i_{2}}\right)\left(\sum_{J_{1}} \sum_{J_{2}} K_{I I, J J}\left(\sum_{j} L^{J_{1}}\left(y_{j_{1}}\right) L^{J_{2}}\left(y_{j_{2}}\right) \sigma_{j}\right)\right) .
$$

This expression is identical to the 1D equivalent in equation (4.8), except that the single summations over $J$ and $I$ have now become double summations over the two grid index directions of the source and receiver nodes $J_{1}$ and $J_{2}$, and $I_{1}$ and $I_{2}$. As stated in Brandt [21] p.26, "the main gain here is not in reducing the number of points, but in the transition to a uniform grid". In this case the transition is to a uniform distribution of boxes with similar grids.

The anterpolation, coarse grid summation, and interpolation steps are easily recognized in equation (4.23) going inward-out of the parentheses. The extension of the MLMIC algorithm for asymptotically-smooth, singular kernels described in Section 4.2.2 from one dimension to multiple dimensions for the case of arbitrary located point sources and receiver points is therefore straightforward.

The formulation of the interaction between source and receiver boxes in the 2D MLMIC method is described by an expression similar to equation (4.23). Instead of using single indices $i$ and $j$ we will use (tensor product) array indices. Figure 4.8 gives an illustration of the elements in the interaction between background grids in 2D space, where coarse grid box sizes are twice the fine grid box sizes. 


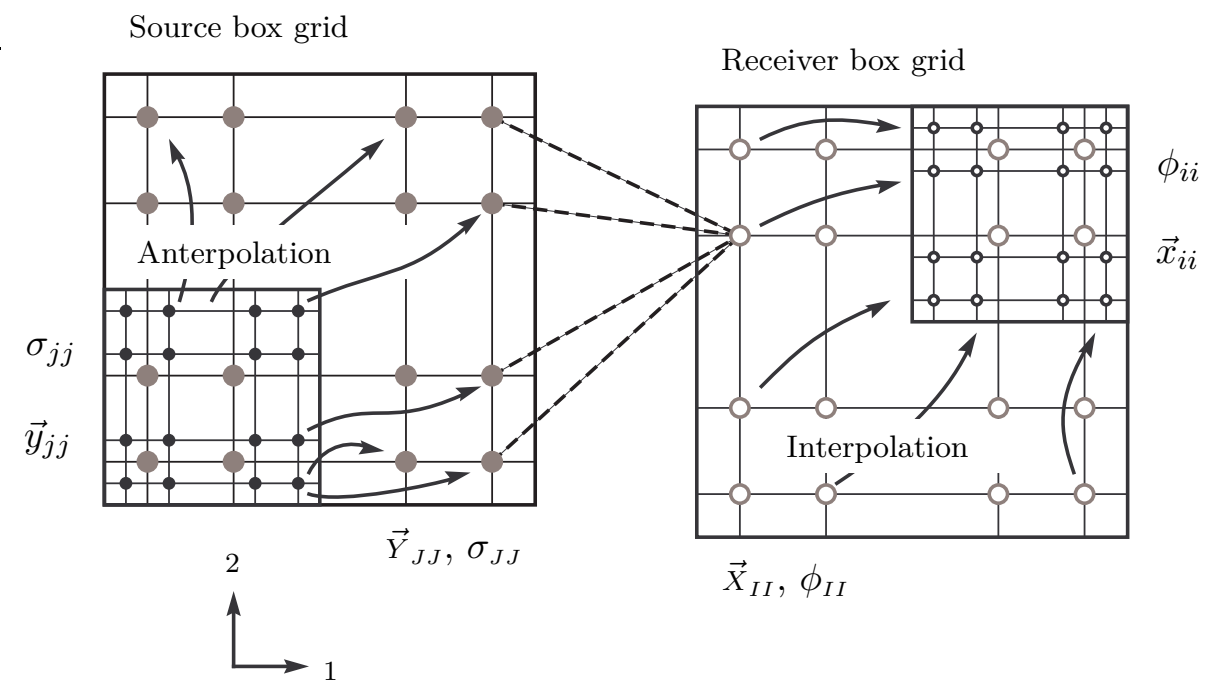

Figure 4.8: Concepts in the MLMIC interaction of fine and coarsened 2D source and receiver box grids.

The approximation of the original summation in equation (4.19) can now be written as

$\phi_{i i} \approx \sum_{I_{1}} L^{I_{1}}\left(x_{i_{1}}\right) \sum_{I_{2}} L^{I_{2}}\left(x_{i_{2}}\right)\left(\sum_{J_{1}} \sum_{J_{2}} K_{I I, J J}\left(\sum_{j_{1}} L^{J_{1}}\left(y_{j_{1}}\right) \sum_{j_{2}} L^{J_{2}}\left(y_{j_{2}}\right) \sigma_{j j}\right)\right)$.

Anterpolation of the source nodes is performed one dimension at the time and likewise the interpolation of the coarser grid data to a the finer grid with smaller boxes is done for consecutive dimensions (see Appendix F). The extension of the MLMIC method from two to three dimensions is straightforward.

\subsubsection{Surface Singularity Distributions}

Up to now we assumed that the source distribution $\tilde{\sigma}(\vec{y})$ in the discretized canonical integral transform (4.2) was concentrated in points $\vec{y}_{j}$. In this section we will discuss the handling of surface distributed sources.

We start the evaluation of equation (4.1), the boundary integral in twodimensional space $\Omega \in \mathbb{R}^{2}$ over boundary $\partial \Omega$, by discretizing the geometry first and subdivide the boundary into non-overlapping panels $\partial \Omega_{k}$ such that $\partial \Omega=\cup \partial \Omega_{k}$. For each panel a piecewise polynomial function for the source 
distribution $\tilde{\sigma}_{k}^{h}(\vec{y})$ over the surface is assumed. Evaluation of the integral in receiver points $\vec{x}_{i}^{h}$ now consists of a summation of the contributions of all individual panels $\partial \Omega_{k}$ and reads

$$
\phi^{h}\left(\vec{x}_{i}^{h}\right)=\phi_{i}^{h}=\sum_{k} \int_{\partial \Omega_{k}} K\left(\vec{x}_{i}^{h}, \vec{y}\right) \tilde{\sigma}_{k}^{h}(\vec{y}) d s_{y} .
$$

In line with the general approach in the MLMIC method in which only kernel function values are required, we will approximate the integral for each panel with an $m_{q}$-point Gaussian quadrature rule which for a general function $f(\xi)$ reads

$$
\int_{-1}^{1} f(\xi) d \xi \approx \sum_{q=1}^{m_{q}} f_{q} w_{q}
$$

and is exact for polynomial functions $f(\xi)$ up to degree $2 m_{q}-1$. In above formula $w_{q}$ are the quadrature weights and $f_{q}=f\left(\xi_{q}\right)$, where $\xi_{q}$ are the Gaussian quadrature point locations. Substitution of the Gaussian quadrature rule in the integral (4.18) over a panel gives

$$
\int_{\partial \Omega_{k}} K\left(\vec{x}_{i}^{h}, \vec{y}\right) \tilde{\sigma}_{k}^{h}(\vec{y}) d s_{y} \approx \sum_{q=1}^{m_{q}} K_{i, q} \sigma_{q}^{h} J_{q} w_{q},
$$

where

$$
K_{i, q}=K\left(\vec{x}_{i}^{h}, \vec{y}_{q}^{h}\right), \quad \sigma_{q}^{h}=\tilde{\sigma}_{k}^{h}\left(\vec{y}_{q}^{h}\right) \quad \text { for } \quad \vec{y}_{q}^{h}=\vec{y}^{h}\left(\xi_{q}\right) \in \partial \Omega_{k},
$$

and $J_{q}$ is the Jacobian determinant $|d s / d \xi|$ of the transformation from $s$ to $\xi$ at point $\vec{y}_{q}^{h}$. Now, substitution of the quadrature approximation (4.27) for the integral over a panel into equation (4.25) gives a similar result as the case with point sources (4.19)

$$
\phi_{i}^{h}=\sum_{j} K_{i, j} \sigma_{j}^{h} J_{j} w_{j}=\sum_{j} K_{i, j} \hat{\sigma}_{j}^{h}
$$

where the summation is over all quadrature points at $\vec{y}_{j}^{h}$ that take the role of concentrated point sources with transformed source strengths $\hat{\sigma}_{j}^{h}$. Notice that this approach automatically allows for higher order panel geometry discretizations, higher order kernel interpolations, and higher order source distributions. For smooth kernels and for the far field of asymptotically smooth kernels we can therefore use the same MLMIC method for surface distributed source strengths as for point sources.

For singular kernels the approximation of the integral over each panel with a Gaussian quadrature rule breaks down when the receiver point $\vec{x}_{i}^{h}$ is in 
the near field of the panel quadrature source points $\vec{y}_{q}^{h}$. Therefore, in the final cluster near field step the exact (or semi-exact) expressions from the original panel method formulation are used. These analytical expressions involve computationally expensive evaluations of functions like $\ln (x)$ and $\arctan (x)$.

The extension of this approach to integration over higher dimensional manifolds $\partial \Omega \in \mathbb{R}^{d}$ with $d \geq 2$ is straightforward with the use of tensor product Gaussian quadrature rules.

\subsubsection{Vector (Component) Kernels}

Up to now the MLMIC method is written for scalar kernel and source functions connected through a simple scalar multiplication operator. The MLMIC method can also be applied to integral transforms where kernel $K(\vec{x}, \vec{y})$, source strength $\sigma(\vec{y})$, and result $\phi(\vec{x})$ can be scalar, vector, or tensor functions. The canonical integral transform in this case is written as

$$
\phi(\vec{x})=\int_{\partial \Omega} K(\vec{x}, \vec{y}) * \sigma(\vec{y}) d s_{y}
$$

in which $*$ is a general notation for the operator at hand. For vector kernel and source functions for example the $*$ symbol could represent the vector dot product or the vector cross product operator. The adaptation of the MLMIC method for smooth or asymptotically-smooth, singular kernels to this more general equation is to apply the scheme for each combination of integrand and operator.

Another approach to deal with vector or tensor components in the integrand is to write out the general integral transform (4.30) into its scalar components and apply the scalar MLMIC method to each of them. For example, an integral with a vector outer product in its kernel such as the Biot-Savart law will result in a matrix vector product like

$$
\vec{\phi}\left(\vec{x}_{i}\right)=\vec{\phi}_{i}=\sum_{j} \vec{K}_{i, j} \times \vec{\sigma}_{j}
$$

which can be written in vector components, with $\vec{\phi}=\left(\phi_{1}, \phi_{2}, \phi_{3}\right)^{\mathrm{T}}, \vec{\sigma}=$ $\left(\sigma_{1}, \sigma_{2}, \sigma_{3}\right)^{\mathrm{T}}$, and $\vec{K}=\left(K_{1}, K_{2}, K_{3}\right)^{\mathrm{T}}$, as follows

$$
\begin{aligned}
& \phi_{1}=\sum K_{2} \sigma_{3}-\sum K_{3} \sigma_{2}, \\
& \phi_{2}=\sum K_{3} \sigma_{1}-\sum K_{1} \sigma_{3}, \\
& \phi_{3}=\sum K_{1} \sigma_{2}-\sum K_{2} \sigma_{1} .
\end{aligned}
$$


The MLMIC scheme in this case is applied to three different scalar kernels, each with two different source strength distributions, where the setup of the boxes only needs to be done once.

When the kernel contains a vector inner product, for example the dipole perturbation potential surface integral, the resulting summation is

$$
\phi\left(\vec{x}_{i}\right)=\phi_{i}=\sum_{j} \vec{K}_{i, j} \cdot \vec{\sigma}_{j},
$$

which can be constructed from its vector components by

$$
\phi=\sum K_{1} \sigma_{1}+\sum K_{2} \sigma_{2}+\sum K_{3} \sigma_{3} .
$$

When the result of the integral transform (4.30) is a vector function it is often possible to rewrite the equation as the gradient of a scalar equation. For example the Biot-Savart kernel in equation (4.31) can be written in terms of the gradient of the dipole potential equation which has a form like equation (4.33). This is a computationally more efficient approach and is the approach we will follow in this thesis, see also Sandak [98]. In our implementation we will use the MLMIC method to determine the velocity potential function at the finest grid level and obtain the gradient of the velocity potential by using the differentiated form of barycentric Lagrange interpolation as described by Berrut and Trefethen [9] and summarized in Appendix F. We then evaluate the interpolation polynomial of the vector components on the finest grid level in the receiver points and add in the final cluster near field step the vector contributions from the panels using the analytical form of the singular kernel integrals over the panels (see for example Katz and Plotkin [63]).

\subsection{Implementation}

In the current 3D MLMIC implementation we use cubes to fill the domain and use the same polynomial order $p$ for the interpolation in receiver and source boxes in each direction and on all grid levels. Each box is given a Chebyshev node distribution of the first kind. The near field criterion (see equation (4.12)) is set to $d_{\mathrm{nf}}=1$, and the coarsest grid level consists of at most 3 boxes in each direction giving a total of 27 boxes. Each box at a coarser grid level consists of 8 'child' boxes at the finer grid level. The same box decomposition of space is used for the receiver and source side.

A $2 \mathrm{D}$ representation of the box setup and the source boxes $\mathcal{B}^{J}$ contributing to the result in a single receiver block $\mathcal{B}^{I}$ at the same grid level is shown 
in Figure 4.9. The final cluster near field contribution (equation (4.17)) comes from the source points or from the panels from which they originate. In this setup the free parameters are the polynomial interpolation order $p$ and the size $h$ of the boxes at the finest grid level.

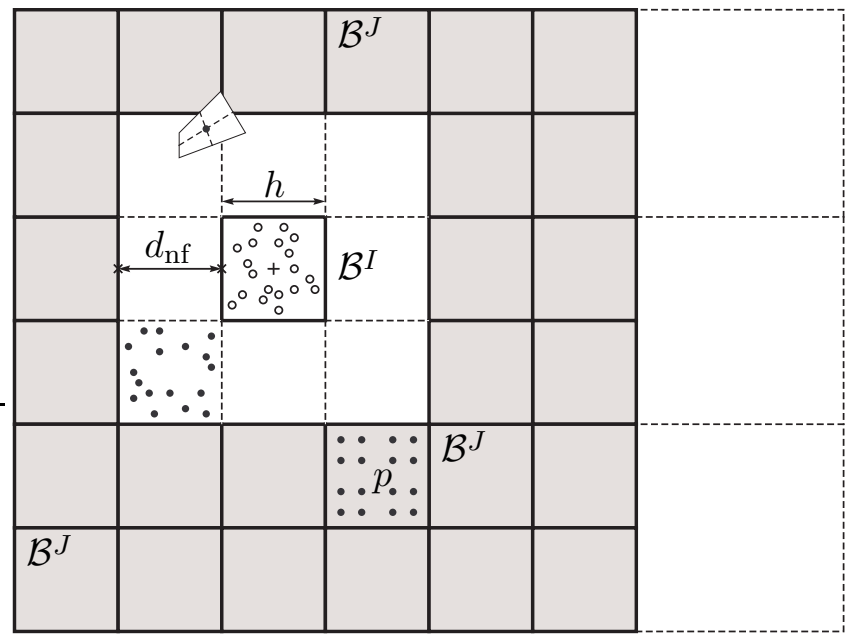

Figure 4.9: Active source boxes $\mathcal{B}^{J}$ interacting with receiver cube $\mathcal{B}^{I}$ on the same grid level. Here a ring of $d_{\mathrm{nf}}=1$ source boxes outside the receiver box defines the perimeter of its near field.

The kernel values involving the nodes in the receiver and source boxes are precomputed and because the kernel functions of interest are translation invariant only the source boxes around a single reference receiver cube have to be considered.

The geometric symmetry in the cube decomposition of space is exploited similar to the approach by Messner, Bramas, Coulaud, and Darve [74]. This allows us to reduce the number of precomputed interactions between source and receiver boxes considerably. For near field criterion $d_{\mathrm{nf}}=1$ only the kernel values of the source cubes shown in Figure 4.10 interacting with the receiver cube have to be computed. This geometric symmetry and the symmetry in the kernel functions makes it possible to combine the (transformed) source strength vectors of related cubes and call optimized library functions for matrix-matrix multiplications instead of a sequence of less efficient matrix-vector multiplications.

Figure 4.11 gives an example of how symmetry is utilized in 2D for a kernel function that depends on distance between nodes only. Here the particular interaction that we want to take into account is between source node $(1,2)$ 


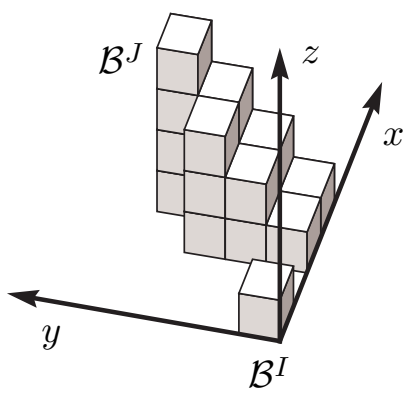

Figure 4.10: Reduced number of precomputed source box kernels for a single receiver cube $\mathcal{B}^{I}$ by exploitation of geometric and kernel symmetries.

in box $\mathcal{B}^{J}$ and receiver node $(2,4)$ in $\mathcal{B}^{I}$. This interaction is indicated by the thick black arrow between the two nodes in the boxes. The symmetry in the kernel and in the geometry makes that this particular interaction is identical to that of receiver node $(3,4)$ in $\mathcal{B}^{I}$ and node $(4,2)$ in the source box in the precomputed domain. Moreover, the multitude of symmetry planes make it possible in this example to relate all interactions indicated with a thin dotted line arrow to the same precomputed interaction. It is possible, through permutations of the source box and receiver box node orderings, to relate all interactions between $\mathcal{B}^{J}$ and $\mathcal{B}^{I}$ with precomputed ones.

In the implementation the interaction between the source box in the precomputed domain and the reference receiver box $\mathcal{B}^{I}$ is computed at the start of a simulation and stored as a matrix for later use. In the MLMIC coarse grid summation step the source strength vectors of all corresponding source boxes are permuted and collected, after which a matrix-matrix multiplication is performed. The result is transformed to correct order and added to the partial result for box $\mathcal{B}^{I}$.

\subsection{MLMIC Verification}

In this section we demonstrate on the basis of model problems that the error in integral values $\phi_{i}^{h}$ and the computational time in the MLMIC method is controlled through the choice of polynomial order and the finest grid box size.

First we will distribute point sources with predefined source strengths over a surface in 3D space and use the same points with a tiny offset as receiver 


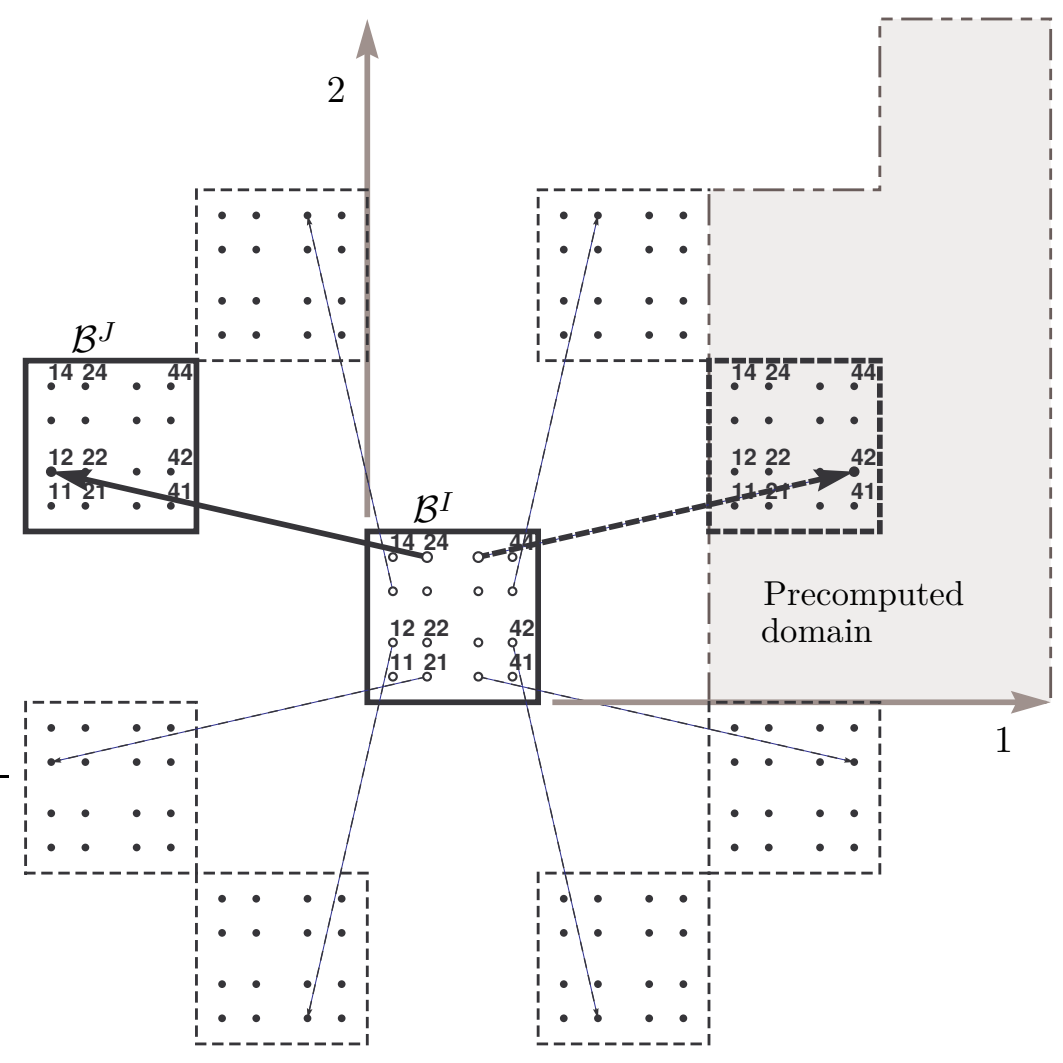

Figure 4.11: Corresponding interactions due to symmetry in geometry for a scalar kernel function dependent on relative distance. The near field criterion is $d_{\mathrm{nf}}=1$.

points. Both the dipole kernel function of equation (3.9) and the source kernel function of equation (3.10) used in the panel method are tested. For both kernel functions we compare the solution from the MLMIC method with a direct evaluation using point receivers and sources. The final MLMIC cluster near field step will use the same direct evaluation. Differences in the solution therefore only come from the anterpolation, coarse grid summation, and interpolation steps in the MLMIC method. For the tests all simulations were performed with a sequentially running code in order to get consistent CPU timings.

Next the MLMIC method is used to perform the solid angle test for a tri-axial ellipsoid as discussed in the verification of the panel method in Section 3.5.2. The final cluster near field step uses the analytical panel integral expressions. In this particular setup this step does not contribute to the error in the solid angle result. This test will give an indication when 
the error incurred by the MLMIC method is of the same order as the error in the far field approximation in the panel method. In that situation the error levels off, and increasing polynomial order or decreasing finest grid level box size does not result in a further decrease in error.

\subsubsection{Source and Dipole Potentials}

In this test we start with a tri-axial ellipsoid with semi-axes $(4,2,1)$ and a total of 16,384 panels (128 along the x-axis, and 128 along the circumference) and double the size and number of panels in x-direction until we have an ellipsoid with semi-axes $(128,2,1)$ and a total of 524,288 panels, now with 4096 panels along the x-axis. This situation, where the addition of extra panels results in an increase in domain size in one direction, is similar to the case of a growing wake downstream of a wind turbine.

We use the panel areas as source strength and panel midpoints as the cloud of point sources. The receiver points have a slight offset from point sources. The finest grid level box size was set to $h=0.25$ for the first set of simulations. The polynomial order was varied from 3 to 7 and the results are compared with the results from the direct evaluation. Figure 4.12 shows the results for the 3D source potential kernel, that is $K(\vec{x}, \vec{y})=1 / r$ with $r=|\vec{r}|$, and $\vec{r}=\vec{x}-\vec{y}$.

The computational work as function of problem size $N$ is shown in Figure 4.12a and from the figure we can deduce that the cost of the direct evaluation is of $\mathcal{O}\left(N^{2}\right)$ and that the MLMIC method in this setup is of $\mathcal{O}(N)$, as was already obtained theoretically in Section 4.2.2. Notice that especially for the lower polynomial orders large reductions in computational time result from the MLMIC method.

Figure $4.12 \mathrm{~b}$ shows the accompanying relative $\mathrm{L}_{2}$-norm (see Section 3.5.1) of the errors in integral values $\phi$ as incurred by the MLMIC method as function of polynomial order $p$. For interpolation of kernels with bounded higher order derivatives the maximum error is $\epsilon \in \mathcal{O}\left(h^{p}\right)$ (see Appendix F), so that $\ln (\epsilon) \propto p$ for fixed box size $h$. This linear dependence of the logarithm of the error on interpolation order $p$ is apparent in Figure 4.12b. The same figure shows a weak dependence of the error on problem size $N$. Here we defined the relative $\mathrm{L}_{2}$-norm error $\epsilon_{\text {rel }}$ as

$$
\epsilon_{\mathrm{rel}}=\frac{\mathrm{L}_{2}\left(\phi_{\text {MLMIC }}-\phi_{\text {direct }}\right)}{\mathrm{L}_{2}\left(\phi_{\text {direct }}\right)} \text {. }
$$

The same tests are performed for the 3D dipole potential kernel $K(\vec{x}, \vec{y})=$ $(\bar{n} \cdot \vec{r}) / r^{3}$ and the results are shown in Figure 4.13a. Due to the three 
component kernel all CPU times in Figure 4.13a are larger than for the single component source kernel shown in Figure 4.12a. As a side note, the CPU times for the direct approach and for the MLMIC method for $p=6$ were used for Figure 4.2 at the start of the chapter, though plotted on a linear scale. Figure $4.13 \mathrm{~b}$ shows that in this test case the error is only a function of polynomial order $p$ and independent of problem size $N$.

The tests are repeated for a two times larger box size $h=0.50$ at the finest grid level and the results are shown in Figure 4.14 and Figure 4.15. The trends are the same as before $(h=0.25)$ but the sensitivity to polynomial order $p$ is smaller as here the final near field contribution dominates the computational time. For the source potential we can see that for interpolation polynomial orders $p \leq 5$ the finer box size $h=0.25$ gives shorter CPU times and that for larger polynomial orders it is advantageous to have the larger box size. A smaller box size gives a smaller near field and thus a faster determination of the final near field contribution. However, a smaller box size also gives rise to more grid levels and could, especially for the higher order interpolations, lead to an imbalance in the number of source points in a cluster and the $p^{3}$ nodes per box at the finest grid level. For example, for the given geometry and box size $h=0.25$ the average number of points in a box at the finest grid level was 15 , whereas for $h=0.50$ it was 60 .

Figure 4.16 shows the relative contributions to the total MLMIC simulation time of the grid setup, the far field evaluation, and the near field evaluation. The results are for interpolation order $p=6$ and concern the evaluation of both the source and the dipole potential integrals. Notice that the time needed for the MLMIC grid setup is only a fraction of the total simulation time in all the cases considered. 


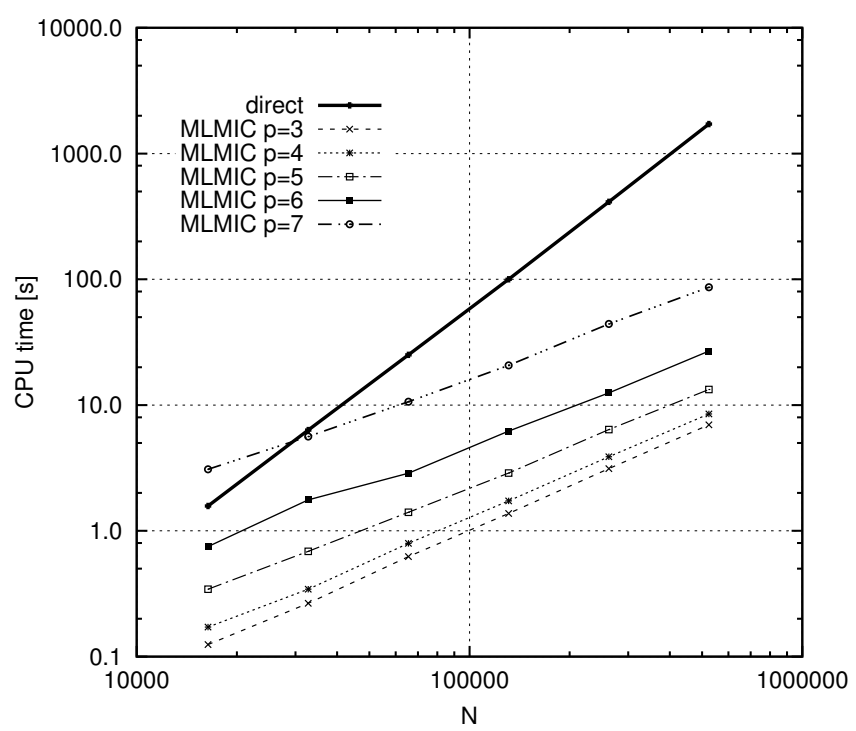

(a) CPU time as function of problem size $N$.

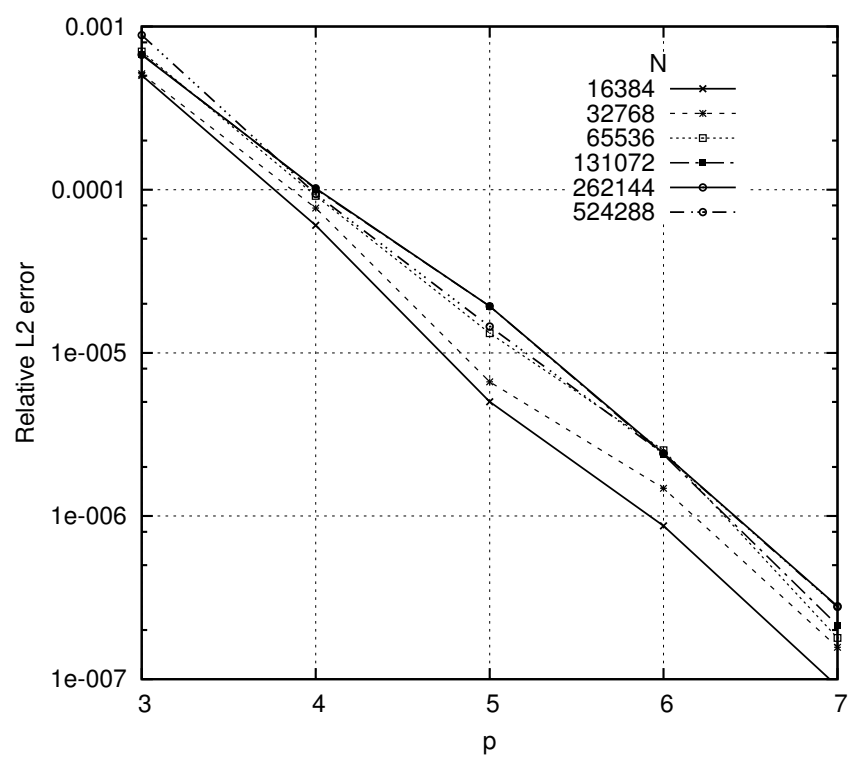

(b) Relative $L_{2}$-norm errors as function of polynomial order $p$.

Figure 4.12: MLMIC method CPU time and errors for the 3D source potential kernel. Source points are distributed on a set of tri-axial ellipsoids of varying length and the finest grid box size is $h=0.25$. 


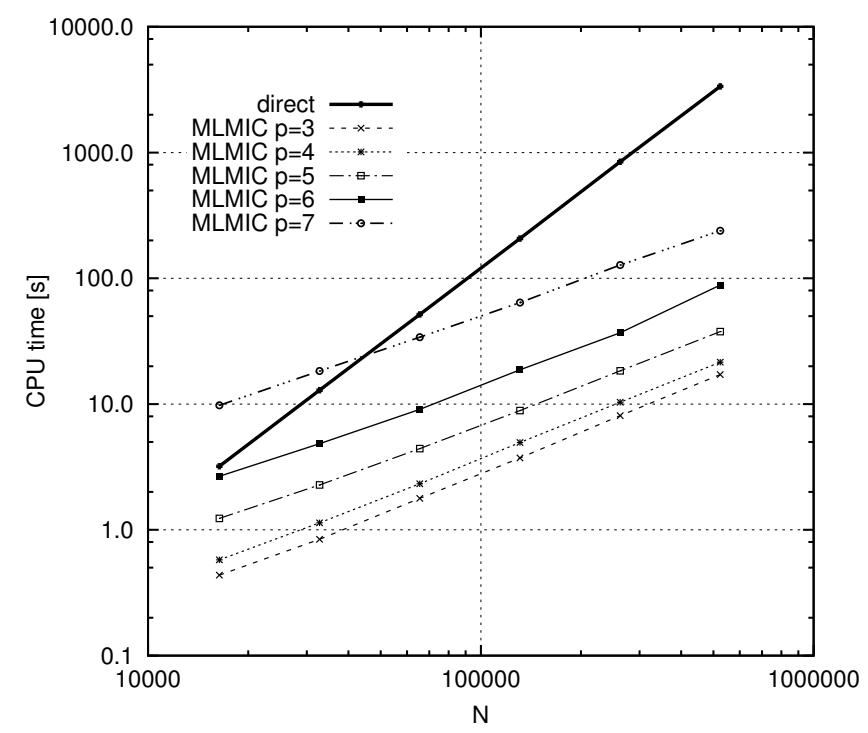

(a) $C P U$ time as function of problem size $N$.

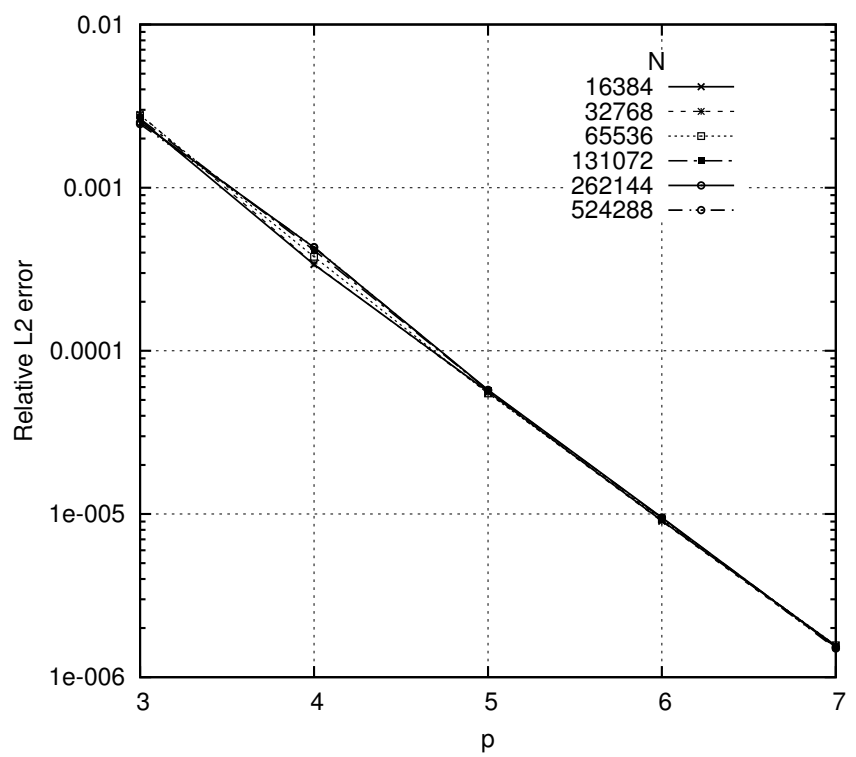

(b) Relative $L_{2}$-norm errors as function of polynomial order $p$.

Figure 4.13: $M L M I C$ method CPU time and errors for the 3D dipole potential kernel. Source points are distributed on a set of tri-axial ellipsoids of varying length and the finest grid box size is $h=0.25$. 


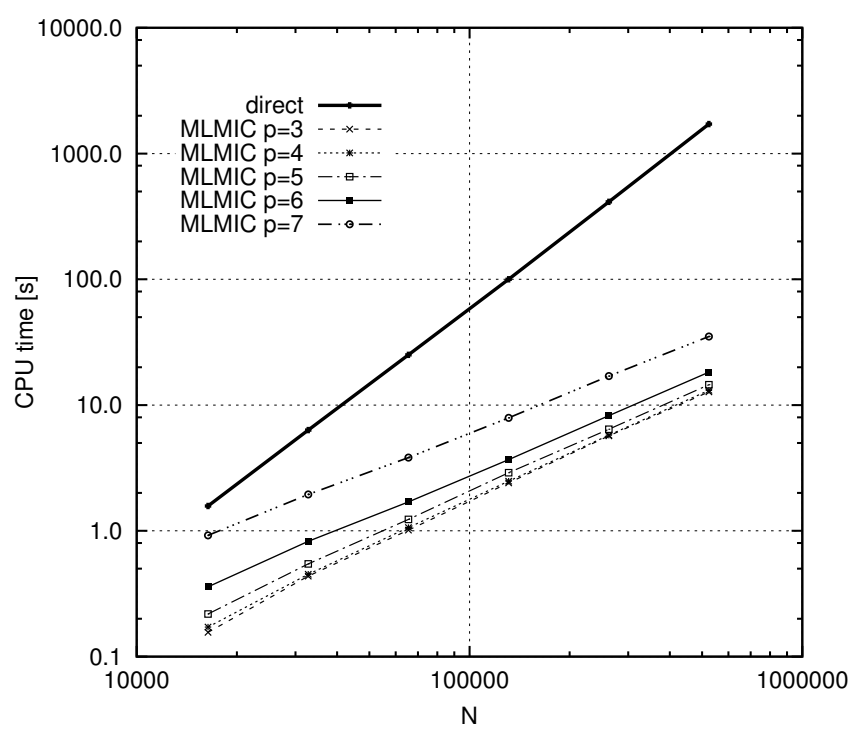

(a) CPU time as function of problem size $N$.

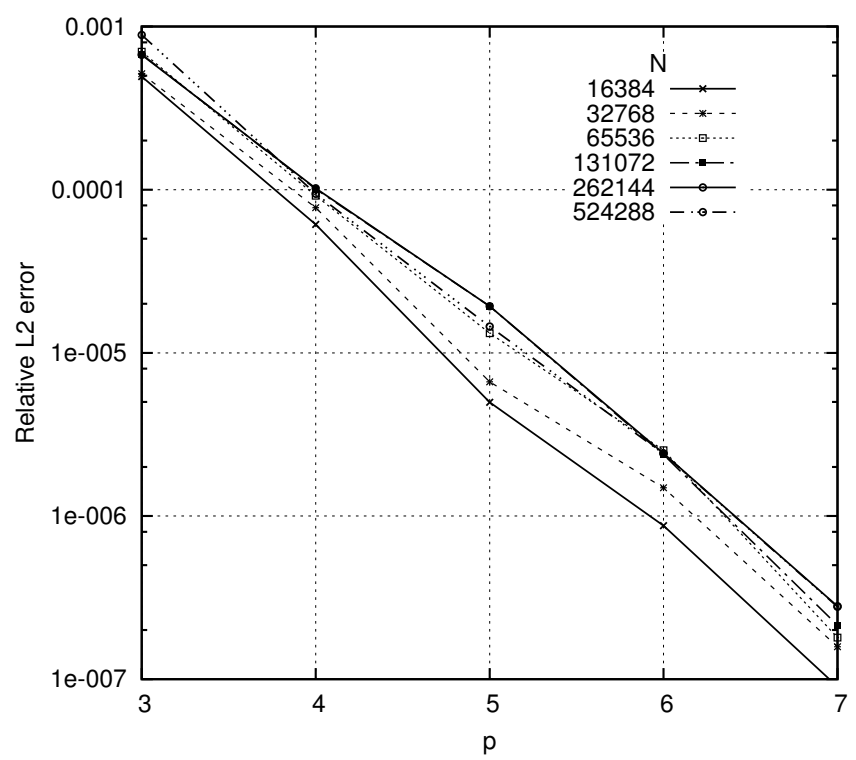

(b) Relative $L_{2}$-norm errors as function of polynomial order $p$.

Figure 4.14: MLMIC method CPU time and errors for the 3D source potential kernel. Source points are distributed on a set of tri-axial ellipsoids of varying length and the finest grid box size is $h=0.50$. 


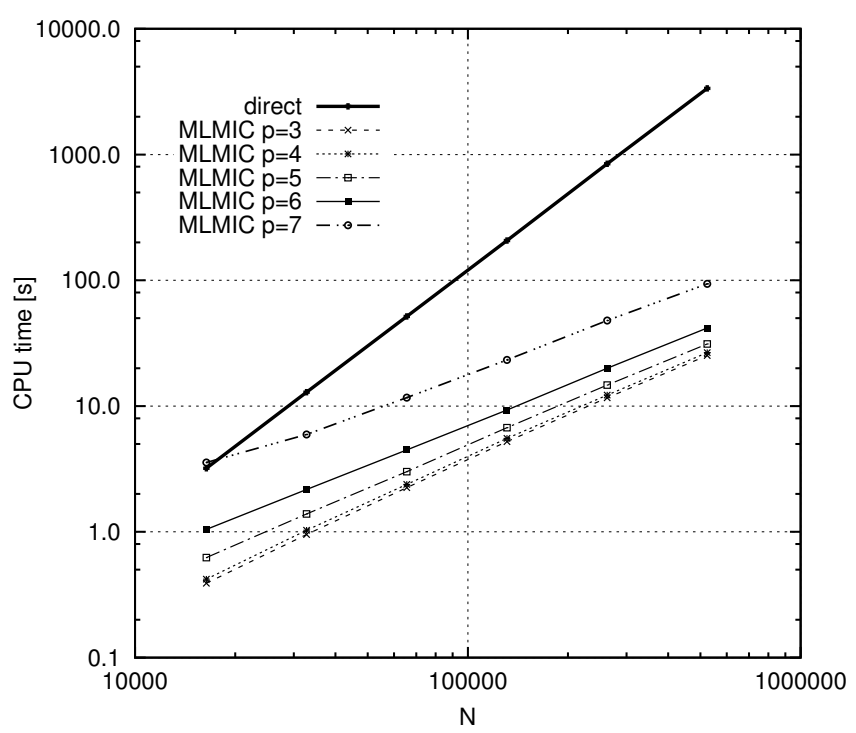

(a) CPU time as function of problem size $N$.

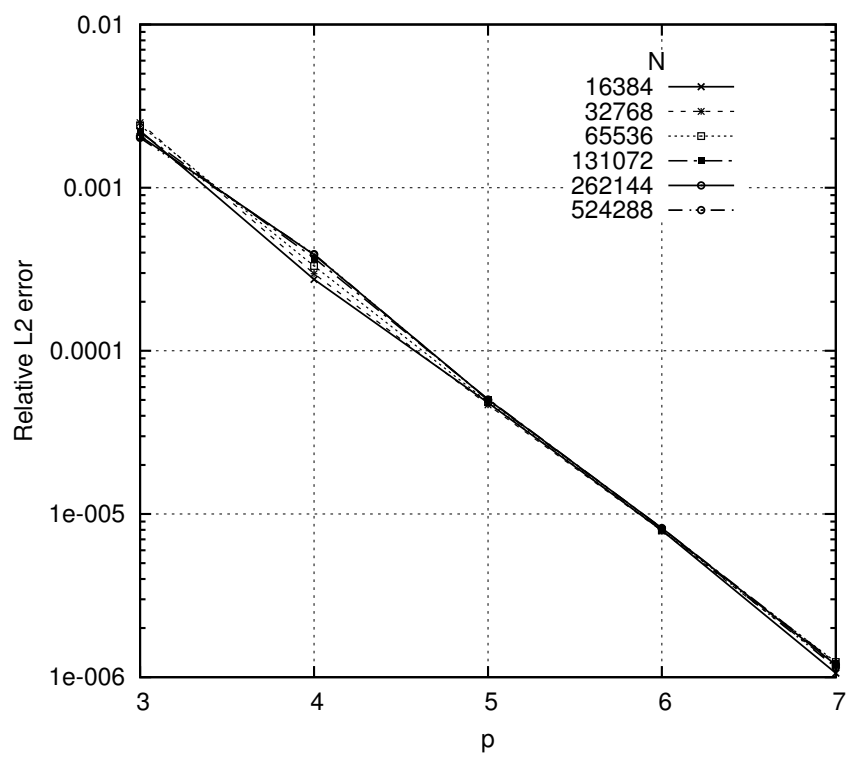

(b) Relative $L_{2}$-norm errors as function of polynomial order $p$.

Figure 4.15: MLMIC method CPU time and errors for the 3D dipole potential kernel. Source points are distributed on a set of tri-axial ellipsoids of varying length and the finest grid box size is $h=0.50$. 


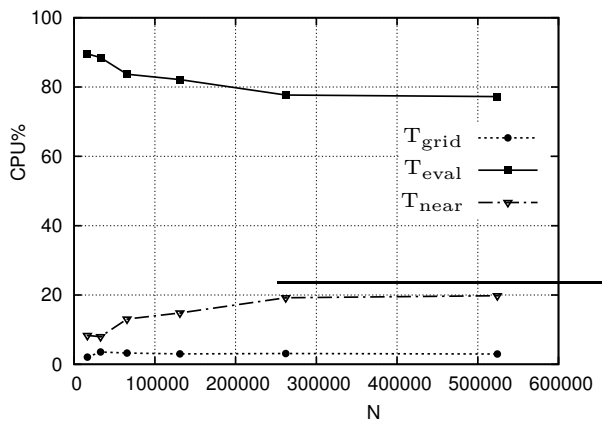

(a) Source potential, $h=0.25$.

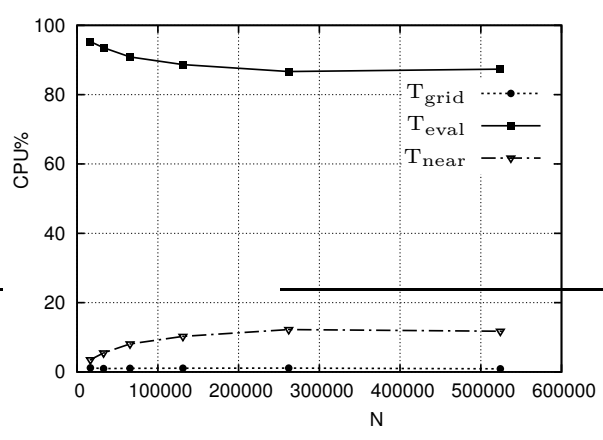

(c) Dipole potential, $h=0.25$.

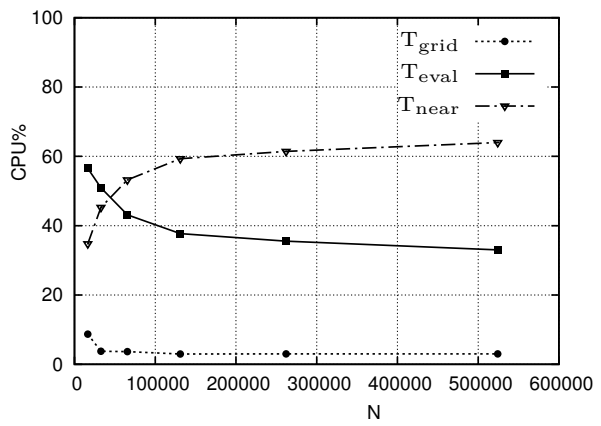

(b) Source potential, $h=0.50$.

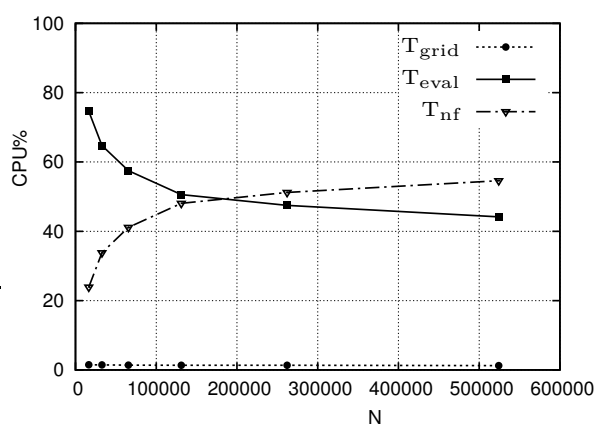

(d) Dipole potential, $h=0.50$.

Figure 4.16: Relative contributions of grid setup, far-field evaluation, and nearfield evaluation to the total MLMIC simulation time for the evaluation of source and dipole potential integrals and polynomial order $p=6$. 


\subsubsection{Solid Angle Tests}

For the verification of the MLMIC method in a more realistic setting the solid angle test for a tri-axial ellipsoid as discussed in Section 3.5.2 is used. The analytical panel integral expressions from that implementation are used in the final cluster near field step of the MLMIC method. In this particular setup the near field step does not contribute to the error in the solid angle result. The error we incur will then only be from the use of point source approximations plus the errors introduced by the polynomial interpolation of the kernel function in the MLMIC method. The test will give an indication when the error incurred by the MLMIC method is of the same order as the error in the far field approximation in the panel method. In that situation the error levels off, and increasing polynomial order $p$ or decreasing finest grid level box size $h$ will not result in a further decrease in error and the goal of the MLMIC method is achieved.

The tests will be performed for the $(4,2,1)$ semi-axes ellipsoid with the number of panels increasing from 1024 to 262,144 by doubling the number of panels from $32 \times 32$ in each surface direction simultaneously. The polynomial order will be increased from 3 to 7 and the same order will be used for all grid levels. The finest grid cube sizes are selected to be $h=0.2,0.4,0.8$.

Figure 4.17a shows the behavior of the relative error in the solid angle as a function of the polynomial interpolation order for different problem sizes when the finest grid cube size is fixed. We already concluded from Figure 3.11 that for a fixed near field size an increasing number of panels on the ellipsoid surface will result in a smaller error level and this holds in the current MLMIC result when the polynomial order is sufficiently high. The error levels stagnate for each of the problem sizes $N$ from the point where the error due to the point source approximation starts to dominate over the error due to the polynomial interpolation. For larger problem sizes this stagnation occurs at higher polynomial orders.

A similar behavior is observed in Figure 4.17b where now the problem size is fixed and the size of the finest grid level cubes (and thereby the near field size) is varied. A larger near field size will reduce the error in the solid angle computation and stagnation of the error level will start at a higher interpolation order.

This behavior of the error leveling off is also apparent in Figure 4.18a where the relative error in the solid angle is plotted as function of problem size $N$ for a given near field size. The error level for the lower polynomial orders taper off already at the smaller problem sizes where the error is dominated by the MLMIC kernel interpolation. 


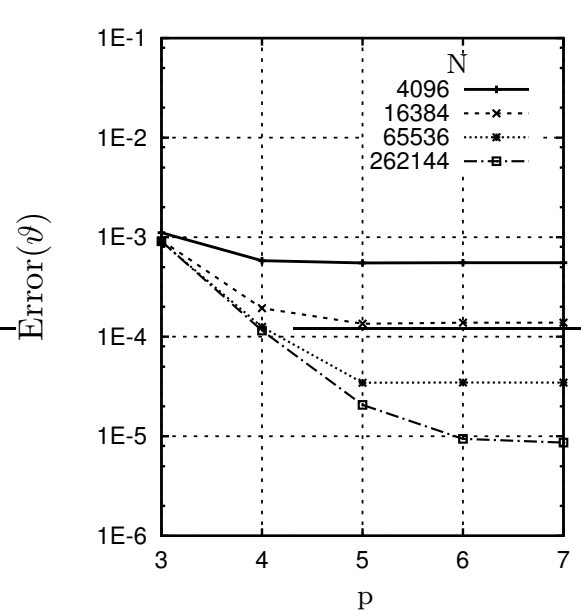

(a) Fixed finest grid cube size $h=0.4$.

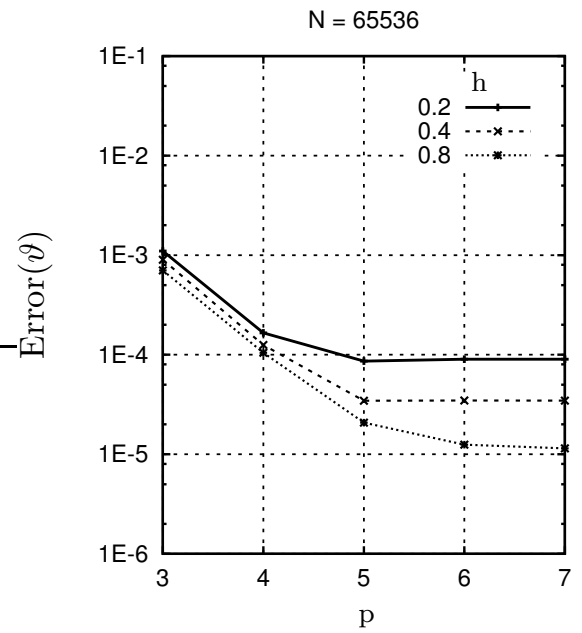

(b) Fixed problem size $N=65536$.

Figure 4.17: Relative $L_{2}$-norm solid angle error as function of polynomial order $p$ for a $(4,2,1)$ semi-axes ellipsoid.

Figure 4.18b shows that for a fixed polynomial order an increasing near field size will shift the error level to lower values but that the error level stagnates from the moment that the error due to the MLMIC interpolation starts to dominate over the error due to the point source approximation.

Both pictures in Figure 4.18 show that given a sufficiently high polynomial order the error is of $\mathcal{O}(1 / N)$ which means that the error reduces quadratically with the panel size as was also observed for the panel method without the MLMIC scheme in Figure 3.13. The error level is then limited by the polynomial interpolation order $p$. 


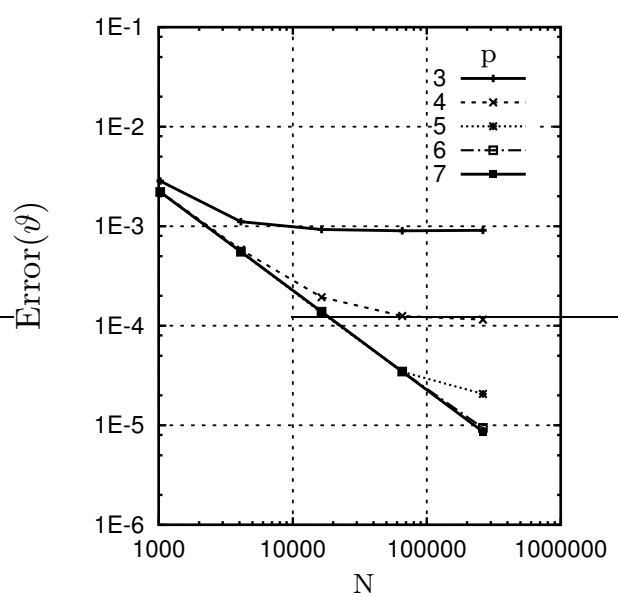

(a) Fixed finest grid cube size $h=0.4$.

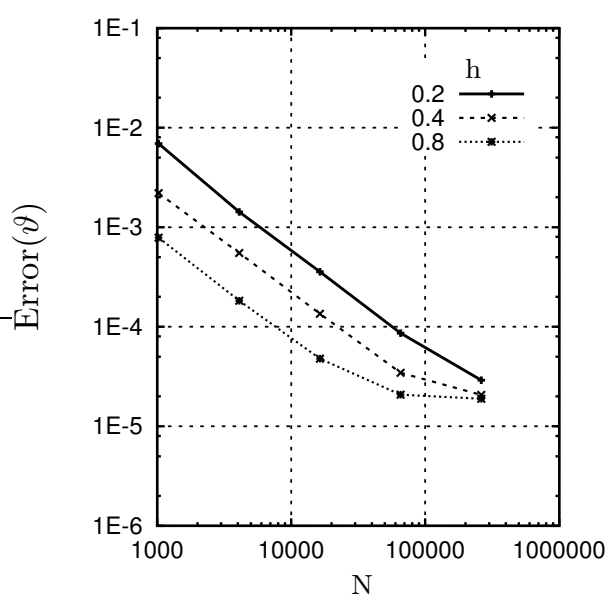

(b) Fixed polynomial order $p=5$.

Figure 4.18: Relative $L_{2}$-norm solid angle error as function of problem size $N$ for a $(4,2,1)$ semi-axes ellipsoid. 


\subsection{Related Work}

In this section we discuss existing multilevel methods used in the fast computation of integral transforms. Having established an understanding of the current multilevel method we now discuss these other algorithms and relate the steps in those schemes with the steps in the MLMIC method.

\subsubsection{Fast Multipole Method}

In his thesis work Greengard [41] in 1988 was the first the reduce the work in three-dimensional gravitational or electrostatic field N-body simulations from $\mathcal{O}\left(N^{2}\right)$ to $\mathcal{O}(N)$ with a controlled error level by his so called Fast Multipole Method (FMM). The FMM algorithm is considered to be one of the important scientific developments of the 20th century and is associated with Leslie Greengard's name and that of his supervisor Vladimir Rokhlin [42],[43],[95].

In the Fast Multipole Method the kernel function is approximated by spherical harmonics series expansions valid in a neighborhood around the expansion point. Like the general Taylor series expansion, the infinite series involves higher order derivatives of the kernel function and the error level is controlled by the number of terms taken into account. A particular feature of spherical harmonics expansion of a potential field is that each of the terms in the series is a solution of the Laplace equation and none of the terms is redundant. Compared to the Taylor series expansion or the MLMIC method, fewer terms are required for a given accuracy (see Morse and Feshbach [78],[79]). However, in some cases the requirement for a potential field and the requirement for higher order derivatives is an undesirable property.

The difference between the FMM algorithm and the MLMIC method described in the preceeding sections is that the kernel function in the FMM is approximated by a function involving a set of (mixed) higher order derivatives in a single expansion point, while the MLMIC method involves just the kernel function itself but in a set of interpolation nodes. The simplicity and applicability of the MLMIC method for general integral transforms is an advantage the scheme has over the FMM algorithm.

The MLMIC method is similar to the FMM algorithm in the sense that both divide space into boxes in which information is contained throughout the steps in the algorithm and that both schemes have the notion of a near field and a far field based on those boxes. The seven steps in the FMM scheme [41],[42] have a corresponding action in the MLMIC method. 
Greengard just numbered these steps but in later publications names and acronyms were attached to some of the steps as indicated in the following list:

- Step 1, Particle-to-Multipole (P2M)

"Form multipole expansions of potential field due to particles in each box about the box center at the finest mesh level."

This step corresponds to the anterpolation of source points to the nodes in the finest grid in the MLMIC method.

- Step 2, Multipole-to-Multipole (M2M)

"Form multipole expansions about the centers of all boxes at all coarser mesh levels, each expansion representing the potential field due to all particles contained in one box."

In the MLMIC method this is the recursive anterpolation of source strengths up to the coarsest grid level.

- Step 3, Multipole-to-Local (M2L) and Local-to-Local (L2L)

"Form a local expansion about the center of each box at each mesh level (but not the finest grid level). This local expansion describes the field due to all particles in the system that are not contained in the current box, its nearest neighbors, or its second nearest neighbors. Once the local expansion is obtained for a given box, it is shifted ... to the centers of the box's children, forming the initial expansion for the boxes at the next (finer) level."

This step is a combination of the coarse grid summation at each grid level and the interpolation between grid levels in the MLMIC method.

- Step 4, Multipole-to-Local (M2L)

"Compute interactions at the finest grid level."

This step corresponds to the coarse grid summation at the finest grid level in the MLMIC method.

- Step 5, Local-to-Particle (L2P)

"Evaluate local expansions at particle positions."

In the MLMIC method this step corresponds to the final interpolation from the finest grid to the receiver points.

- Step 6

"Compute potential (or force) due to near neighbors directly."

Together with the next step this corresponds to the final cluster near field step in the MLMIC method. 
- Step 7

"Add direct and far-field terms together."

The mathematics behind the FMM algorithm are quite involved and the implementation of the theory in a numerical method is far from trivial.

\subsubsection{Panel Clustering Method}

Hackbusch and Nowak [49] in 1989 published their work on the Panel Clustering method. The method makes use of a Taylor series expansion of the kernel function centered around a point at the source (panel) side that is integrated over the panel area. The coefficients in the series relate to moments of various orders over the panel area and only have to be calculated once. Clusters of nearby panels are formed and their expansions are shifted and combined around a common point, and clusters of clusters can be formed subsequently for when the receiver point is further away. This last step is fundamental and distinguishes the Panel Clustering scheme from the use of Taylor series based multipole expansions in the panel method by Hess and Smith [54] in 1967.

The Panel Clustering scheme uses higher-order derivatives of the kernel function but has the advantage over the FMM approach in that it has no operator-dependent series expansion and can be applied for general kernel functions. Moreover, the Panel Clustering scheme differs from the FMM scheme in that it was designed for surface integrals and not for point sources. This drawback however is easily overcome in the FMM scheme with the approach described in Section 4.3.2. At the receiver side no series expansions are used and the Panel Clustering scheme has therefore a somewhat increased computational complexity as compared to the Fast Multipole Method and the MLMIC method.

Expressed in the terms used to describe the MLMIC method, the Panel Clustering technique only uses anterpolation of the source panels to (Taylor series) multipole expansions at the coarser grid level. The summation involves the multipole approximations and the non-coarsened receiver points. In later versions of the Panel Clustering scheme [46] a hierarchical approach was also introduced at the receiver side. Like the FMM scheme the Panel Clustering scheme is used for the far-field influences, that is, when source panels are sufficiently far away from the evaluation point. The near field is accounted for by a direct evaluation of the panel integrals.

The Panel Clustering method was generalized by Hackbusch [45],[47] from a panel based approach to a degree-of-freedom index oriented approach. 
The resulting construction of hierarchical matrices ( $\mathcal{H}$-matrices) is based on trees of index-clusters and leads to a block-structured matrix, where the sub-blocks are low-rank matrices. This generalized approach supports all normal matrix operations like (approximate) addition, multiplication, and matrix inversion. Initially the contruction of the hierarchical matrices was based on Taylor expansions. In later publications [16], [17], [39] [48] the construction of hierarchical $\mathcal{H}^{2}$-matrices included an approach using interpolation of the kernel function. A hierarchy of approximations was used for both the source side and the receiver side of the integral transform (hence the name $\mathcal{H}^{2}$ ). The approach based on interpolation was introduced by Brand and Lubrecht [23] and is discussed in the next section.

\subsubsection{Multi-Level Multi Integration}

The Multi-Level Multi-Integration (MLMI) scheme by Brandt, Lubrecht, and Venner [23],[111] was first published in 1990. One year later Brandt [21] extended the scheme to integral transforms with oscillatory kernels. The MLMI scheme forms the basis of the MLMIC method in this thesis. Prior to these publications the basic idea of the MLMI method was outlined already by Brandt [19] in 1981 and described briefly in [20] in 1987.

In the MLMI scheme the kernel function is approximated with an interpolating polynomial through multiple points whereas the FMM and Panel Clustering approaches spherical harmonics or a Taylor series expansions in a single point are used that rely on higher-order derivatives of the kernel function. This makes the MLMI scheme more readily applicable for general kernel functions.

The MLMI scheme discretizes the domain (surface) of integration directly using a uniform (equidistant) grid. The kernel function in an interval is approximated by an even order $p$ Lagrange polynomial, symmetrically positioned around the interval, that is, a centered quasi-interpolation is used. This gives an expression for the approximate integral over the interval that involves $p / 2$ kernel values to the left and $p / 2$ values to its right. This differs from the MLMIC method in two ways. First, the interpolation in an interval requires a larger region with kernel values that 'moves' with each interval. Consequently, the anterpolation step distributes the source values to larger and larger regions with each grid coarsening step. And secondly, the domain of integration must be smooth to warrant the approximation of the kernel function with a polynomial.

At the coarsest grid level the coarse grid summation is performed. For asymptotically-smooth kernel functions corrections are performed for the 
anterpolation and interpolation steps separately when sources and receivers are in each others near field. The local coarse grid contribution is subtracted from the solution and replaced by the contribution from the next finer grid level. The correction steps differ from the MLMIC method where clusters of source and receiver points on a grid level only interact when their boxes are in each others far field.

The two corrections in the MLMI scheme can be identified in the expression for the discretized boundary integral in one-dimensional space (see also equation (4.8)):

$$
\phi_{i}^{h} \approx \underbrace{\sum_{I}^{I}\left(x_{i}^{h}\right) \underbrace{\left(\sum_{J} K_{I, J}^{H H}\left(\sum_{j} L^{J}\left(y_{j}^{h}\right) \sigma_{j}^{h}\right)\right)}_{\text {Approximation for } \sum_{j} K_{I, j}^{H h} \sigma_{j}^{h}}}_{\text {Approximation for } \sum_{j} K_{i, j}^{h h} \sigma_{j}^{h}} .
$$

The correction step for anterpolation is named the "coarse grid correction" and the correction in the interpolation step is named "fine grid correction". Cast in the form of the MLMIC method discussed in Section 4.2.2, the steps in the MLMI scheme are

\section{Anterpolation}

The source strengths $\sigma_{j}^{h}$ in points $y_{j}^{h}$ are transferred to pseudo-sources $\sigma_{J}^{H}$ located in the surrounding source background grid nodes $Y_{J}^{H}$ by

$$
\sigma_{J}^{H}=\sum_{j} L^{J}\left(y_{j}^{h}\right) \sigma_{j}^{h}
$$

\section{Coarse grid summation}

The kernel values $K_{I, J}^{H H}$ representing the influence of source nodes on receiver background grid nodes are used to perform a matrix-vector multiplication with the vector of pseudo-sources $\sigma_{J}^{H}$ in source nodes $Y_{J}^{H}$ to give the receiver values $\phi_{I}^{H}$ in the receiver background grid nodes $X_{I}^{H}$ through

$$
\phi_{I}^{H}=\sum_{J} K_{I, J}^{H H} \sigma_{J}^{H}
$$

\section{Coarse grid correction}

Replace the contributions from the previous two steps when receiver nodes in $X_{I}^{H}$ are in the near field of source points in $y_{j}^{h}$ with the best 
possible solution in the receiver background grid nodes:

$$
\phi_{I}^{H}:=\phi_{I}^{H}+\sum_{\substack{j \\|2 I-j| \leq m}}\left(K_{I, j}^{H h}-\sum_{J} K_{I, J}^{H H} L^{J}\left(y_{j}^{h}\right)\right) \sigma_{j}^{h} .
$$

\section{Interpolation}

The receiver values $\phi_{i}^{h}$ in points $x_{i}^{h}$ are obtained by interpolation of the receiver values $\phi_{I}^{H}$ in the surrounding nodes $X_{I}^{H}$ of the receiver background grid through

$$
\phi_{i}^{h}=\sum_{I} L^{I}\left(x_{i}^{h}\right) \phi_{I}^{H} .
$$

\section{Fine grid correction}

Replace (part of) the coarse grid correction and its subsequent interpolation with the best possible solution when the receiver point at $x_{i}^{h}$ is in the near field of the source point at $y_{j}^{h}$ :

$$
\phi_{i}^{h}:=\phi_{i}^{h}+\sum_{\substack{j \\|i-j| \leq m}}\left(K_{i, j}^{h h}-\sum_{I} K_{I, j}^{H h} L^{I}\left(x_{i}^{h}\right)\right) \sigma_{j}^{h} .
$$

For the coarse grid summation in equation (4.38) the MLMI scheme can be applied recursively. Note that for convolution-type kernels, when the integration is over all equidistant intervals, and the near field sets can be determined a priori, the coefficients in the coarse grid summation and the two correction steps can be precomputed.

Brandt and Lubrecht [23] hinted in their publication at a cluster variant of the MLMI scheme for general type kernels. Paraphrased, they stated

This correction work can again be reduced to $\mathcal{O}(n p)$ by grouping together cp points (cp values of $i$ ) at a time and by carrying out the corrections for all these cp points over a fixed (independent of i) interval (of length $2 m+c p=\mathcal{O}(p)$ ), instead of the varying interval $(|i-j| \leq m)$ used in (4.41).

This observation was the start for the development of the MLMIC method. At the University of Twente, following the thesis work by Venner [110] in 1991, the MLMI scheme was topic of further research in the area of contact dynamics by Bos [18] in 1995, and Wijnant [118] in 1998. The application of the MLMI scheme for acoustics problems was investigated by Hérnandez Ramírez [51] in 2005. 


\subsubsection{Precorrected-FFT Method}

In 1997 Phillips and White [90] published their multilevel algorithm that uses the fast Fourier transform to efficiently evaluate the matrix-vector product occurring in the inner loop of iterative algorithms for solving electromagnetic boundary integral equations. The algorithm is at best $\mathcal{O}(N \ln N)$ but has the advantage that it can rely on existing optimized FFT implementations. The precorrected-FFT (pFFT) method makes use of a uniform background grid that encompasses the domain of evaluation points and the integration surface. The algorithm relies on the translation invariance of the kernel function. There is no explicit hierarchy of grids in the algorithm; this is part of the FFT method. In the pFFT algorithm four major steps can be distinguished that can be related best to the MLMI method in Section 4.6.3:

\section{Projection}

"Project the panel charges onto a uniform grid of point charges."

This step corresponds to the anterpolation of source points to the finest background grid in the MLMI method. However, in the pFFT algorithm the coefficients in the projection step are determined through an explicit optimization of the potential in test points surrounding a cell. This gives the approximation of the panels (integrals) located in the cell by the set of $p^{3}$ pseudo-charges in the background grid. Like the MLMI algorithm 4.6.3 the grid nodes in a region surrounding the cell are affected and might overlap with other regions with projected panel charges.

\section{Convolution}

"Compute the grid potentials due to grid charges using an FFT."

This step requires one forward and one inverse 3D FFT after which the potential in the receiver grid is obtained. The computational effort of the FFT algorithm is $\mathcal{O}(N \ln N)$. This step is related to the combined anterpolation, coarse grid summation, and interpolation steps in the MLMI algorithm that involve all background grid levels.

\section{Interpolation}

"Interpolate the grid charges onto the panels."

In this step the grid potentials are interpolated to the panels and in case of a Galerkin panel method this step is exactly the adjoint operation of the pFFT projection step. This step is equivalent to the final interpolation step in the MLMI algorithm where the intermediate solution in the evaluation points is obtained. 


\section{Nearby Interactions}

"Directly compute nearby interactions."

The interactions are calculated inaccurately by the FFT convolution step when panels and evaluation points are in close proximity in case of asymptotically smooth kernels. Similar to the MLMI method a correction is applied locally that consists of subtracting the local (near field) variant of the previous three steps for each cell and replacing it with a direct interaction between panels and evaluation points.

Phillips and White mention that this step is expensive to compute but that its cost is incurred just once when the pFFT scheme is part of the inner loop of an iterative algorithm.

The pFFT scheme is applied in a panel method for aerospace applications by Willis [119] and Willis, Peraire and White [120] where it is used for the fast matrix vector product evaluation in a GMRES iterative solver. They cite the high computational setup costs of the pFFT scheme and use a Fast Multipole Method with lower setup costs for the simulation of the dynamics of the wake behind a wing. 


\section{Application}

\subsection{MEXICO experiment}

In 2014 new wind tunnel tests were performed for a 4.5 meter diameter three-bladed model wind turbine in the Large Scale Low Speed Facility (LLF) (see Figure 5.1) of the German Dutch Wind Tunnels (DNW) [15]. This most recent set of measurements was a follow up of the initial measurement campaign [102],[104] in the same wind tunnel in 2006 in the MEXICO (Model EXperiments In COntrolled conditions) project. The combined results from these wind tunnel measurements form a large database of surface pressures, forces, flow velocity vectors, and aero-acoustic data for a range of operational conditions. Among these operating conditions are variations in wind speed, rotational speed, wind turbine yaw angle, and rotor blade pitch angle. This database is used extensively in research and validation studies by a large international consortium of institutes and universities [100],[101].

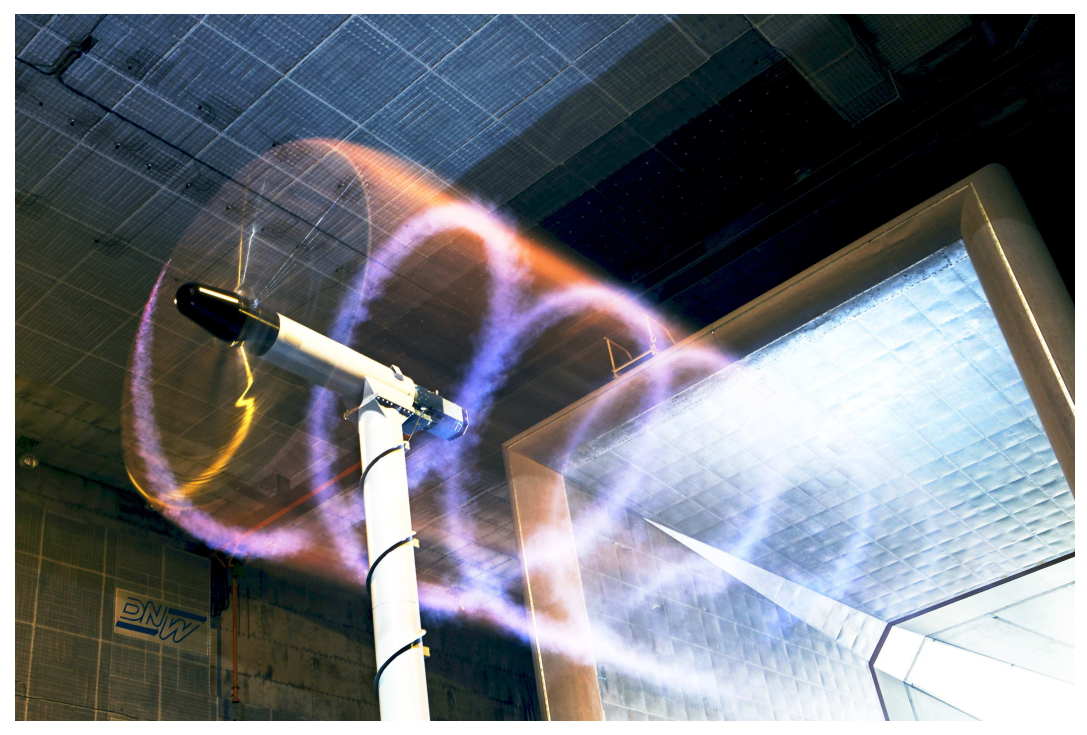

Figure 5.1: Visualization of flow around the MEXICO model wind turbine in the Large Scale Low Speed Facility (LLF) of the German Dutch Wind Tunnels $(D N W)[15]$.

In the "New MEXICO" wind tunnel experiments surface pressure distributions were measured at sections at $25 \%, 35 \%, 60 \%, 82 \%$, and $92 \%$ radius. 
Table 5.1: Selected "New MEXICO" test cases.

\begin{tabular}{c|r|c}
\hline$U_{\infty}$ & \multicolumn{1}{|c|}{$\lambda$} & run \\
\hline \hline $10.0 \mathrm{~m} / \mathrm{s}$ & 10.0 & 265 \\
$14.7 \mathrm{~m} / \mathrm{s}$ & 6.8 & 266 \\
$24.1 \mathrm{~m} / \mathrm{s}$ & 4.2 & 268 \\
\hline
\end{tabular}

We select three axial flow conditions (i.e. zero yaw angle) to compare our simulated pressure distributions with the experimental results in the database. The conditions are at a constant rotational speed of 425.1 RPM, giving a rotor tip speed of approximately $U_{\text {tip }}=100.1\left[\mathrm{~m} \cdot \mathrm{s}^{-1}\right]$. Selected wind speeds are 10.0, 14.7, and $24.1\left[\mathrm{~m} \cdot \mathrm{s}^{-1}\right]$. This corresponds to the three different tip speed ratios $\lambda=U_{\text {tip }} / U_{\infty}$ given in Table 5.1.

The blade of the MEXICO rotor is comprised of three airfoil sections shown in Figure 5.2. Each of the airfoils is used in a specific region of the blade: the part of the blade with the DU91-W2-250 airfoil extends from $20.0 \%$ to $45.56 \%$ radius, the part of the blade with the Ris $\varnothing$ A2-21 airfoil covers the region from $54.44 \%$ to $65.56 \%$ radius, and the part of the blade with the NACA64-418 airfoil is from $74.44 \%$ to $100 \%$ of the rotor radius. Figure 5.3 shows the three parts of constant airfoil cross-section. These parts are connected through transition elements. Figure 5.3 also shows the 5 sections of the pressure measurements.

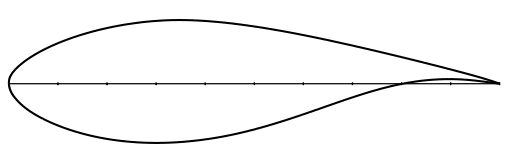

(a) DU91-W2-250 airfoil.

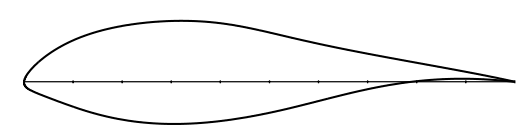

(b) Risø A2-21 airfoil.

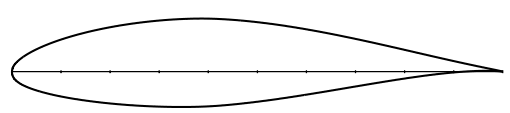

(c) NACA64-418 airfoil.

Figure 5.2: Airfoil sections used in the MEXICO rotor blade.

A validation study was performed at NLR by Ten Pas [89] in 2016 for the state-of-the-art numerical simulation method ENSOLV [66] that is based on the Navier-Stokes equations. Data from the MEXICO experiments were used in the validation of ENSOLV for wind turbine applications. Part of the ENSOLV validation was concerned with the pressure distributions at five radial positions for the three operating conditions listed above in Table 5.1. We include the results from that study in our comparison. 


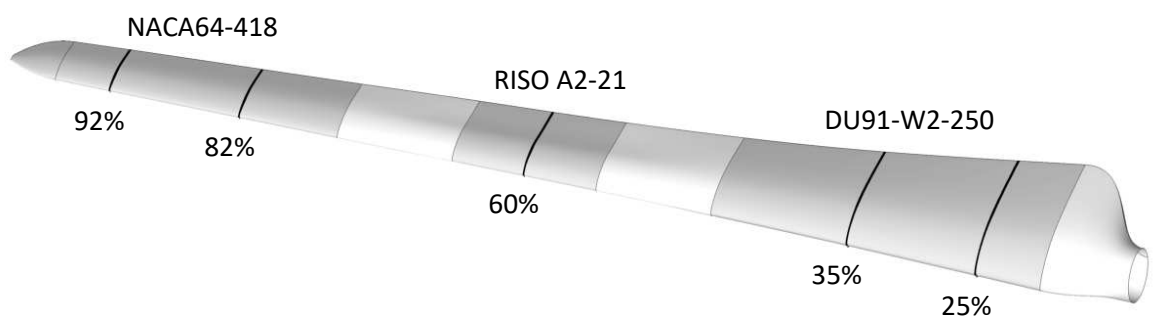

Figure 5.3: Regions of constant airfoil cross-section of the MEXICO rotor blades.

ENSOLV is a high order finite-volume method that uses multi-block structured grids. In the ENSOLV computations a fully turbulent boundary layer was assumed and represented with a nonlinear explicit algebraic Reynoldsstress model based on the modified $k-\omega$ turbulence model by Kok [65]. The axial periodicity in the geometry and the flow conditions has been exploited to reduce the computational domain to one rotor blade. The blade surface was represented by a grid of 164 cells in radial direction and 128 cells around the circumference of the blade cross sections. The wind turbine nacelle was included in the computational domain. The total number of cells was approximately 9 million. Constructing the block decomposition of the space around the configuration such that a high quality grid could be generated was the most labor-intensive task and took about a week. Once a block decomposition is obtained the generation of the grid takes less effort. The ENSOLV computations have been performed on a high end computer system at NLR with typical run times of half an hour per flow condition.

For the numerical simulations using the panel method the three blades of the MEXICO wind turbine are included but the wind turbine nacelle (see Figure 5.4) is excluded. The blades feature zero trailing edge thickness. For one blade a fine grid is used which consists of 92 panels in radial direction and 120 panels in cross-section circumferential direction. A coarser grid is used for the other two blades which consists of 46 panels in radial direction and 30 panels for the blade cross-section. Figure 5.4 displays the wake of the blade with the finest grid. All three wake surfaces extend 2000 panels downstream. In total this grid configuration consists of 13,800 panels for the three blade surfaces and 368,000 panels for the three wake surfaces.

This discretization is used in simulations for the MEXICO rotor at design conditions by both the conventional panel method and the panel method using the MLMIC scheme. The computation of the velocity induced at all wake panel corner points (Equation (3.14)) by all 381,800 panels at the body and wake surfaces takes 6990 seconds $(\approx 1.9 \mathrm{hr}$ ) for a single time 


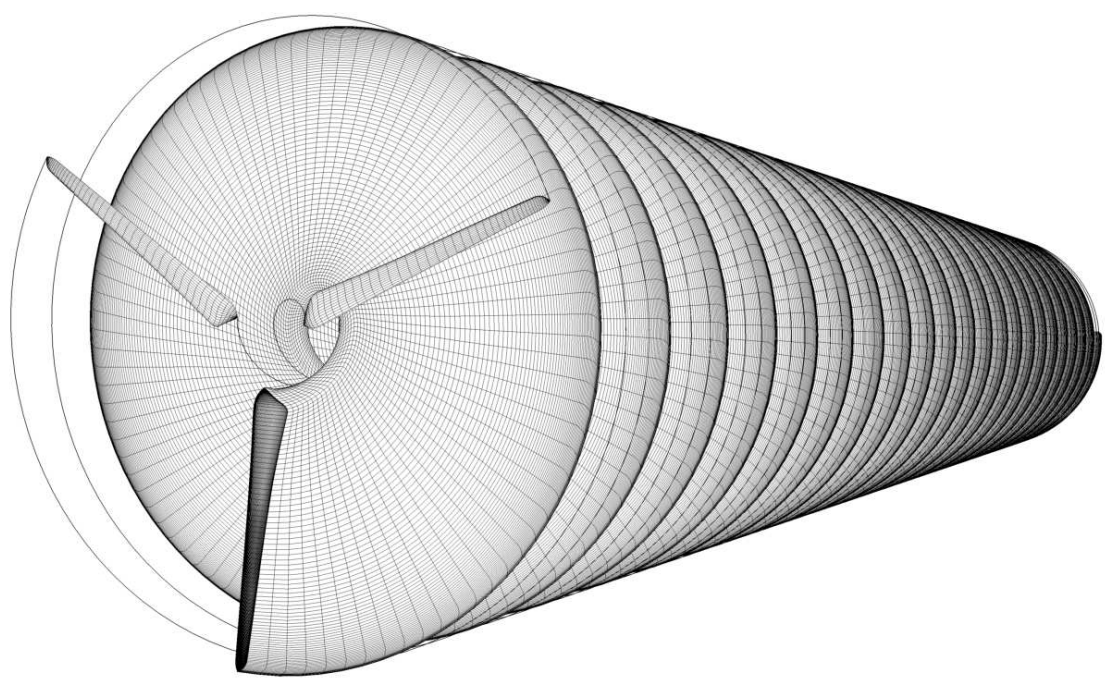

Figure 5.4: Discretized MEXICO rotor geometry and wake surfaces used in the panel method. For clarity only one wake surface is displayed. The total number of panels for the three blades is 13,800 and the three wake surfaces are discretized with 368,000 panels in total.

step with the conventional panel method on a general-purpose personal computer. The computation on the same computer of the same problem with the MLMIC scheme, with interpolation order $p=4$ and finest grid box size $h=0.4[\mathrm{~m}]$, takes less than 46 seconds per time step. This gives a speedup factor of over 150 for this grid configuration.

The MLMIC method was also applied with the same parameters in the computation of the influence of the wake panels at the panels on the blade (i.e. the right-most term in Equation (3.8)). Here the computing time was reduced from 258 seconds per time step for the conventional panel method to 4 seconds per time step for the MLMIC method, giving a speedup factor of 64 . Figure 5.5 compares the surface pressure distribution at $25 \%$ radius of the MEXICO rotor blade for these two simulations. For the sections towards the tip the differences in computed surface pressure distributions are smaller and indiscernible at this scale of the plot.

The application of the MLMIC method for the evaluation of the velocity in the wake and for the evaluation of the right-hand side of the linear system of equations (3.8) makes the Krylov iterative method currently the most time-consuming part of the panel method. For the grid configuration above, a time step takes 564 seconds CPU time. The Krylov method consumes 513 CPU seconds: more than $90 \%$ of the CPU time. An obvious future 
improvement is to replace the current dense matrix-vector multiplications in the Krylov iterations that represent the left-hand side of (3.8) with MLMIC integral transform evaluations.

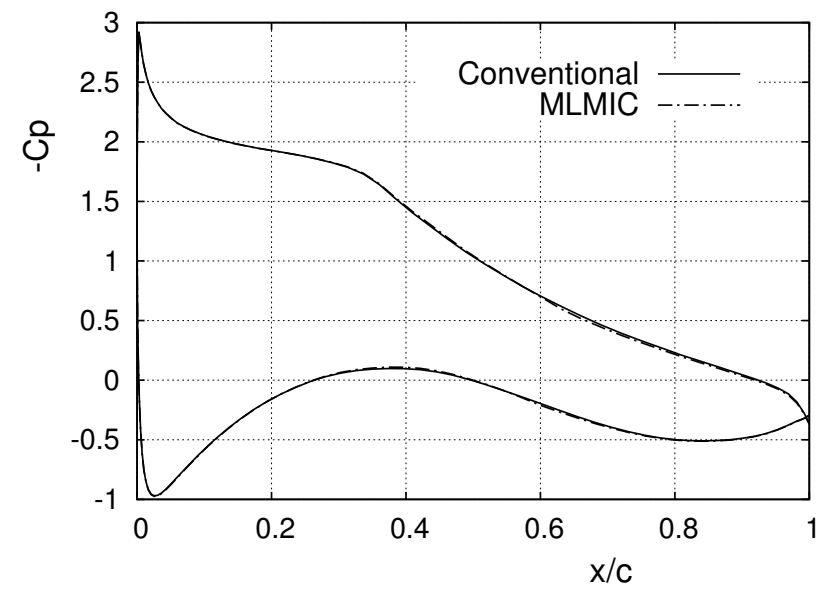

Figure 5.5: Comparison of computed surface pressure distribution at 25\% radial position, obtained by conventional panel method and by panel method with MLMIC computation of the wake influence on the rotor. The MLMIC results are obtained with interpolation order $p=4$ and finest grid box size $h=0.4[\mathrm{~m}]$. The MEXICO rotor operates at design condition: wind velocity $14.7\left[\mathrm{~m} \cdot \mathrm{s}^{-1}\right]$ and tip speed ratio $\lambda=6.8$.

\section{High tip speed ratio}

Figure 5.6 shows the pressure distributions computed using the panel method and the ENSOLV method, compared to the MEXICO wind tunnel experiment for wind speed $10.0\left[\mathrm{~m} \cdot \mathrm{s}^{-1}\right]$ and tip speed ratio $\lambda=10.0$. This tip speed ratio is higher than the tip speed ratio at design conditions and as a consequence the local incidence of the blade sections is lower.

The surface pressure distributions for the NACA64-418 airfoil sections at $92 \%$ and $82 \%$ radius are shown in Figures $5.6 \mathrm{a}$ and $5.6 \mathrm{~b}$. The panel method is based on an inviscid flow model and shows, as expected, a lower pressure at the suction side of the airfoil. The effects of viscosity are represented in the ENSOLV method and as a result the computed surface pressure distributions at the suction side of the blade are closer to the experimental data. At the pressure side of the blade sections the boundary layer is much thinner and the result from the panel method is in very good agreement with the experimental data. 
The Ris $\varnothing$ A2-21 airfoil section at $60 \%$ radius is an airfoil with a different character of the surface pressure distribution (see Figure 5.6c): a higher adverse pressure gradient between about $40 \%$ and $60 \%$ chord along the blade suction side. Both numerical simulation methods show a substantial lower pressure than the experiment. Note that the difference between the ENSOLV result and the experimental data is larger than the difference between the result of the panel method and that of ENSOLV. At the pressure side of the blade section the results of both numerical simulation methods are in excellent agreement with the experimental data.

For the DU91-W2-250 airfoil section at 35\% and that at 25\% radius a consistent pattern is shown between the results of the panel method and that of ENSOLV, see Figures 5.6d and 5.6e: the panel method produces lower pressures along the airfoil suction side. The pressure gradient in the ENSOLV results are reduced by the effects of viscosity. At the pressure side of the blade the two numerical simulation methods give similar results, except near the trailing edge where effects due to the boundary layer become more pronounced. The surface pressure distribution from the experiment shows erratic behavior for the section at $25 \%$. This can be attributed to the sensors measuring absolute pressures and the low dynamic pressure at the innermost sections [15],[89], resulting in larger error bounds.

\section{Design tip speed ratio}

Figure 5.7 shows the pressure distributions from the panel method, the ENSOLV method, and the MEXICO wind tunnel experiment for the rotor design condition: wind speed $14.7\left[\mathrm{~m} \cdot \mathrm{s}^{-1}\right]$ and tip speed ratio $\lambda=6.8$.

A similar pattern as for the high tip speed ratio emerges: the results of the panel method show a pressure along the suction side of the airfoil that is somewhat lower than that from ENSOLV and that from the experiment. Along the pressure side the ENSOLV and panel method results are in very close agreement for the first half of the airfoil where effects due to the boundary layer are expected to be small.

\section{Low tip speed ratio}

Figure 5.8 shows the surface pressure distributions for the rotor operating at $24.1\left[\mathrm{~m} \cdot \mathrm{s}^{-1}\right]$ wind speed and at a low tip speed ratio $\lambda=4.2$. The experimental data indicate that the rotor blade experiences boundary layer separation for all sections considered for the aft $50 \%$ to $70 \%$ of the blade suction side. 
The panel method does not represent the boundary layer nor flow separation. Therefore it continues to have a lower pressure along the suction side of the airfoil. In this case the differences with the results from ENSOLV and from experimental data are large. While still under predicting the effects of viscosity, ENSOLV captures most of the flow separation.

We conclude by stating that for the case for which the rotor blade operates near design condition, or more general, in conditions for which the boundary layer remains attached, the results of the panel method are in good agreement with results from both the experiment and the ENSOLV numerical solution. An even better agreement is possible if the effects of the boundary layer are taken into account. This case will be argued further in Chapter 6 in the discussion on future developments.

To support this statement we extract the MEXICO rotor blade NACA64418 cross-section at $82 \%$ radius. For this airfoil section we perform two numerical simulations with XFOIL [31],[30]. In the first XFOIL simulation we assume the flow to be inviscid and select an angle-of-attack of $5.905^{\circ}$ that gives the same minimum pressure as the result from the 3D panel method for the MEXICO rotor in design condition at $82 \%$ radius (see Figure $5.7 \mathrm{~b}$ ). The second simulation is at the same angle-of-attack but with XFOIL in "viscous mode" for a fully turbulent boundary layer at Reynolds number $R e=650,000$ in which an integral boundary layer method accounts for the effects of the boundary layer on the surface pressure distribution.

Figure 5.9 compares the three numerically simulated surface pressure distributions with the experimental data. Clearly, including the influence of the boundary layer on the surface pressure distribution gives a much better agreement with the experimental data along the suction side of the airfoil. 


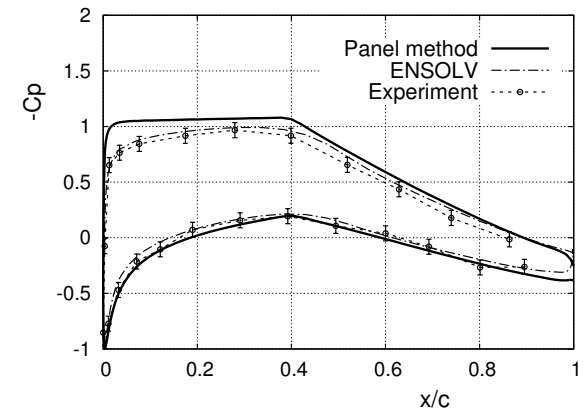

(a) 92\% Rotor radius, NACA64-418.

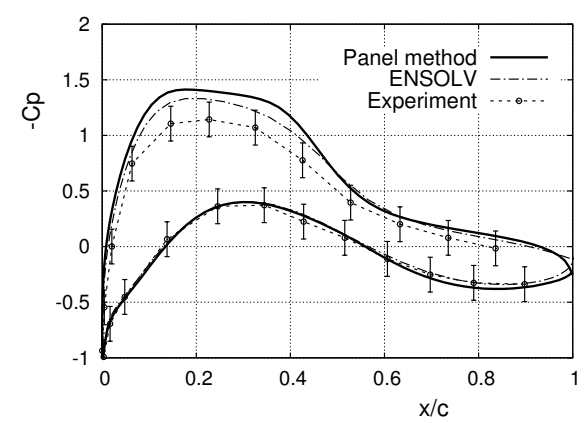

(c) 60\% Rotor radius, Risø A2-21.

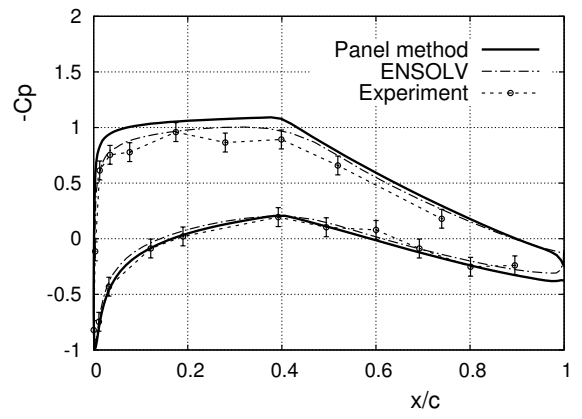

(b) $82 \%$ Rotor radius, NACA64-418.

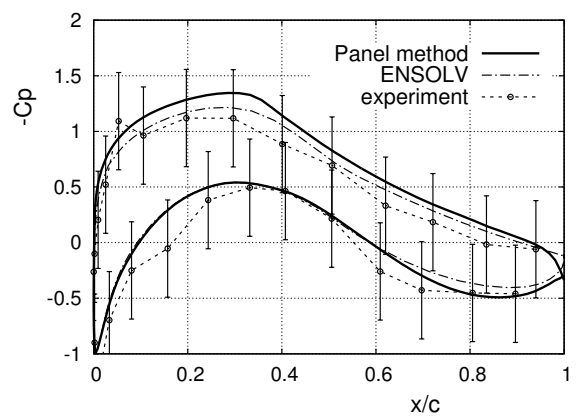

(d) 35\% Rotor radius, DU91-W2-250.

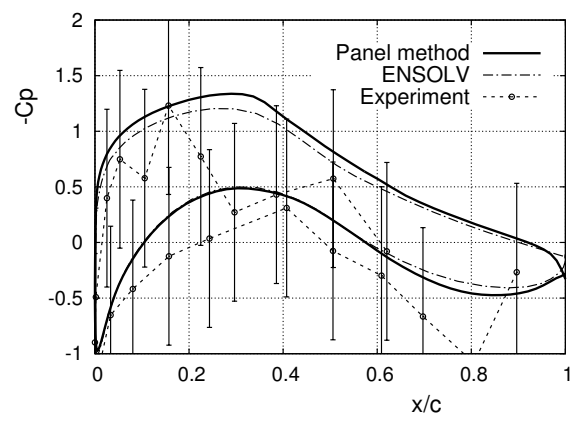

(e) 25\% Rotor radius, DU91-W2-250.

Figure 5.6: MEXICO rotor in uniform onset flow velocity of $10.0\left[\mathrm{~m} \cdot \mathrm{s}^{-1}\right]$ operating at tip speed ratio $\lambda=10.0$. Comparison of measured surface pressure distributions with numerical results from panel method and from ENSOLV, a state-of-theart numerical simulation method that solves the Reynolds-averaged Navier-Stokes equations. 


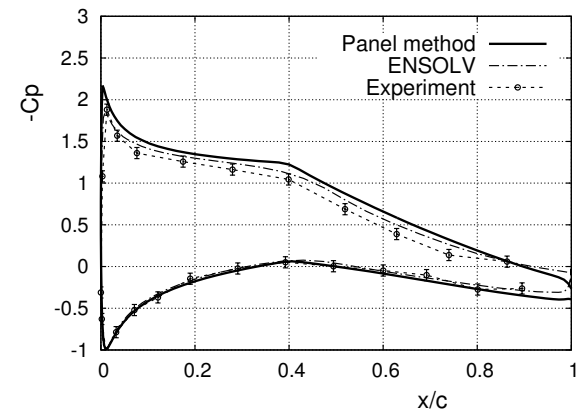

(a) 92\% Rotor radius, NACA64-418.

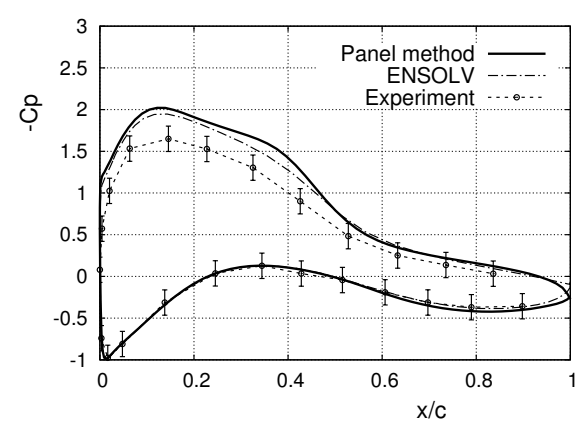

(c) 60\% Rotor radius, Risø A2-21.

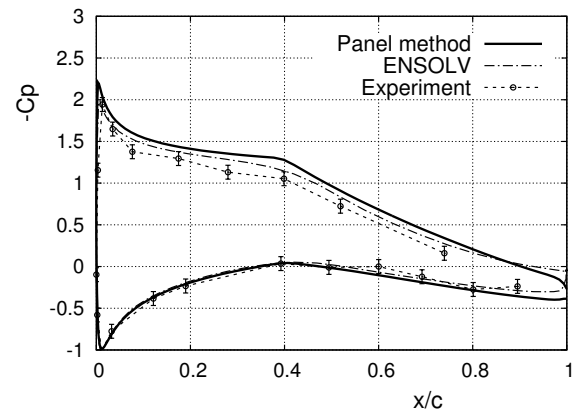

(b) $82 \%$ Rotor radius, NACA64-418.

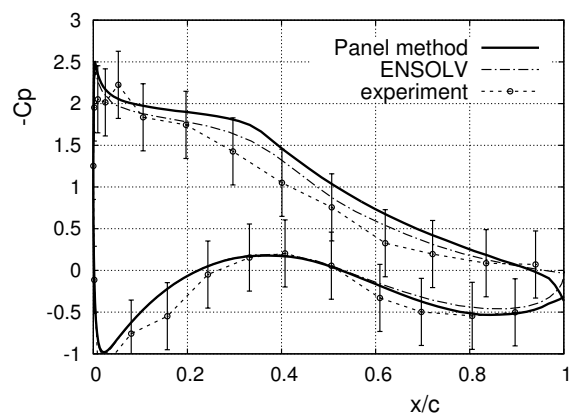

(d) 35\% Rotor radius, DU91-W2-250.

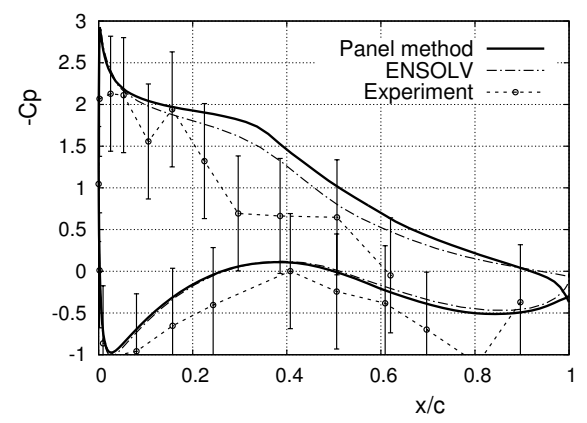

(e) 25\% Rotor radius, DU91-W2-250.

Figure 5.7: MEXICO rotor in uniform onset flow velocity of $14.7\left[\mathrm{~m} \cdot \mathrm{s}^{-1}\right]$ operating at tip speed ratio $\lambda=6.8$ (design conditions). Comparison of measured surface pressure distributions with numerical results from panel method and from ENSOLV, a state-of-the-art numerical simulation method that solves the Reynolds-averaged Navier-Stokes equations. 


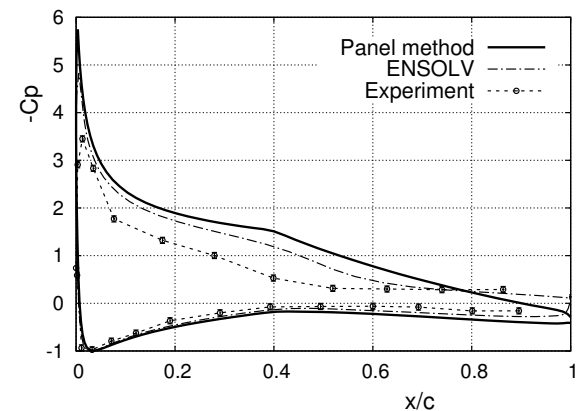

(a) 92\% Rotor radius, NACA64-418.

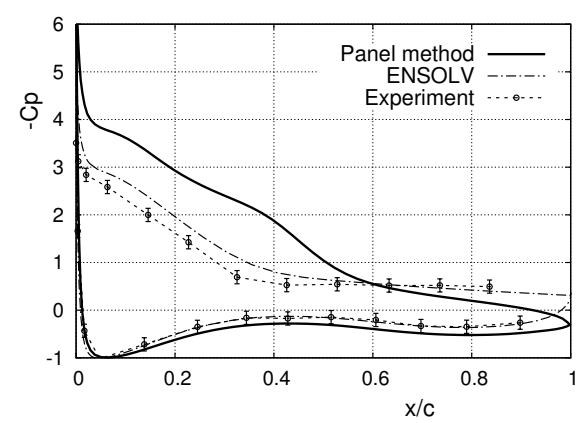

(c) 60\% Rotor radius, Risø A2-21.

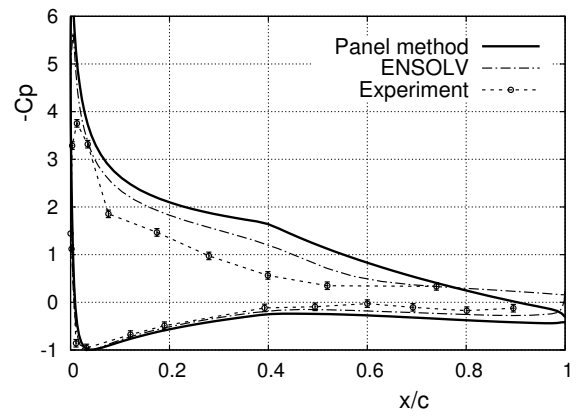

(b) $82 \%$ Rotor radius, NACA64-418.

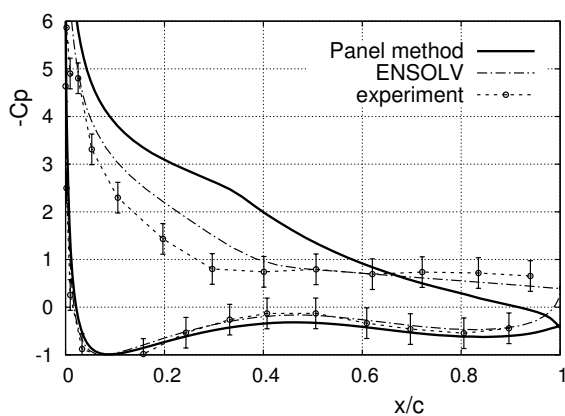

(d) 35\% Rotor radius, DU91-W2-250.

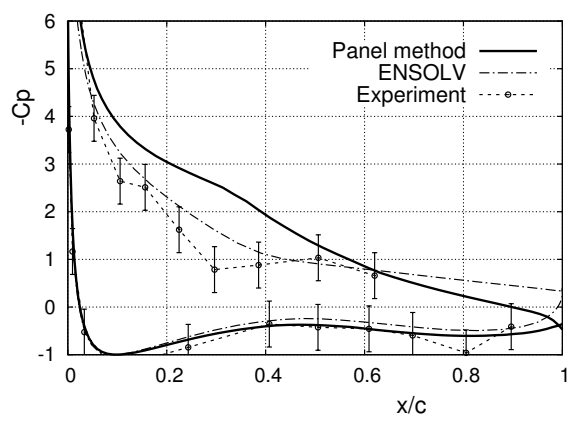

(e) 25\% Rotor radius, DU91-W2-250.

Figure 5.8: MEXICO rotor in uniform onset flow velocity of $24.1\left[\mathrm{~m} \cdot \mathrm{s}^{-1}\right]$ operating at tip speed ratio $\lambda=4.2$, Comparison of measured surface pressure distributions with numerical results from panel method and from ENSOLV, a state-of-theart numerical simulation method that solves the Reynolds-averaged Navier-Stokes equations. 


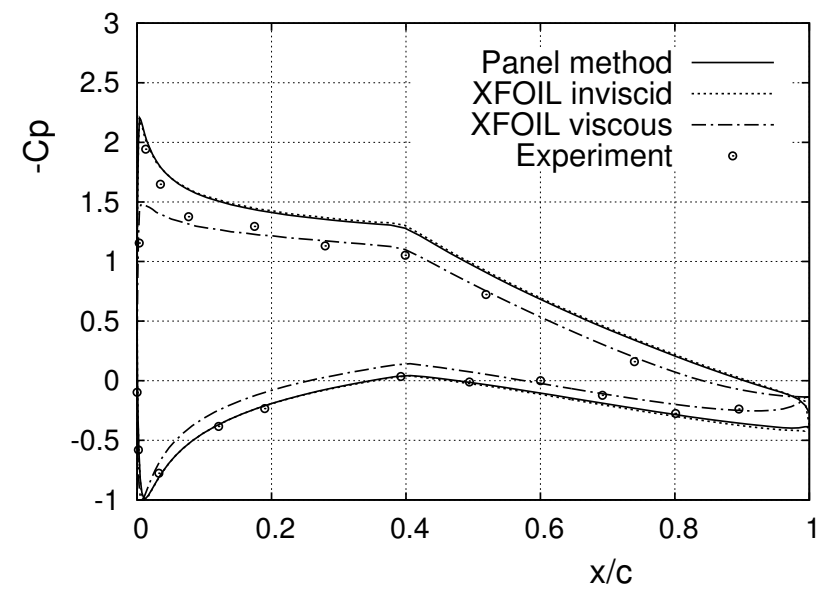

Figure 5.9: MEXICO rotor at design condition. Comparison of the measured surface pressure distribution at $82 \%$ radial position with results from the $3 D$ panel method, from the 2D XFOIL code [31],[30] in inviscid mode, and from XFOIL with fully turbulent boundary layer at $\alpha=5.905^{\circ}, \mathrm{Re}=650,000$. 


\section{Conclusions and Outlook}

\subsection{Conclusions}

This work set out to significantly reduce the computing times of a conventional panel method for the numerical simulation of the deformation of the wake behind a wind turbine and the influence of the wake on the aerodynamic loads acting on the rotor blades. This has been achieved by the developed MLMIC scheme as is demonstrated for the implemented loworder panel method.

This work repositions panel methods in the computational landscape as valuable medium fidelity computational design method for wind turbine engineering.

The basic problem is that for the numerical simulation of wind turbine rotor flows with a conventional panel method the computational burden grows quadratically with the number of degrees-of-freedom $N$ in the representation of singularity distributions on the rotor and wake surfaces. The developed MLMIC method reduces the $\mathcal{O}\left(N^{2}\right)$ computational complexity of the conventional approach to $\mathcal{O}(N)$, i.e. it achieves a speedup of the computation times that grows linearly with problem size.

This speedup is achieved through to the introduction of an interpolating function for the kernel function in the integrand of the integral representation combined with the introduction of a hierarchy of boxes and coarsened grid levels in the volume surrounding the wind turbine. This setup makes it possible to apply the scheme in cases of highly irregular surfaces as occur in the wake of a wind turbine.

The interpolation of the kernel introduces an error that can be controlled by the order of the interpolating polynomial and by the size of the interpolation interval (i.e. the box size) on the finest grid level. As long as the error due to the interpolation is of the same order or smaller than the error in the original (conventional) panel method discretization, this approximation is of no consequence to the order of accuracy of the method.

We compare our results for the pressure distribution at five radial positions to the results from the new MEXICO wind tunnel measurements for three operating conditions, and include the results from ENSOLV, a state-of-theart CFD method based on the Reynolds-averaged Navier-Stokes equations. For the panel method we discretize the three blades of the rotor using a total 
of 13,800 panels and using 368,000 panels to represent the wake surfaces attached to the blades. The results of the panel method compare well with the experimental data and the results of the CFD method for two cases in which the aerodynamic flow conditions can be considered benign. For a third flow condition the flow is separated at the suction side of the blade for the larger part of the blade surface and the assumption of inviscid flow in the panel method is not valid anymore and the comparison breaks down. In these simulations employing the panel method the computation of the influence of the wake on the rotor blades is 64 times faster with the MLMIC scheme than with the conventional panel method. For the computation of the velocity at the wake nodes, used to determine the deformation of the wake within each time step, the MLMIC method achieves a speedup factor of 150 .

A further speed up of the panel method is possible: the current multiplication of a dense matrix and a vector in the iterative Krylov solver can be replaced with MLMIC integral transform evaluations. The present matrixvector multiplication consumes about $90 \%$ of the total CPU time for a time step involving the grid configuration described above.

\subsection{Outlook}

The achieved $\mathcal{O}(N)$ simulation time for the MLMIC method makes it feasible to consider new developments involving the application of the panel method in time-dependent flow conditions as occurring for wind turbines at normal operating conditions. The ultimate goal is to arrive at a fast "medium fidelity" method for the simulation of wind turbine aerodynamics for use in an engineering environment. Such method bridges the gap between the low-fidelity BEM methods and the computationally expensive CFD methods and makes a balanced combination of short problem turnaround times and higher accuracy. There are two major topics that must be considered, namely modeling the boundary layers and fluid-structure interaction:

\section{Modeling the boundary layer}

The assumption of inviscid flow in the panel method gives reasonable results when the effect of fluid viscosity is confined to a very thin layer next to the surface of the configuration. In practice a wind turbine in normal operating conditions experiences aerodynamic forces governed by non-linear effects due to effects of viscosity, invalidating this assumption of inviscid flow. Including a model for the threedimensional boundary layer flow will enhance the predictive capability 
of the panel method considerably (see e.g. [31],[32]).

A solution can be found in viscous-inviscid interaction (VII) technology (see [71],[108]) in which a viscous flow model for the boundary layer is coupled in strong interaction with an inviscid flow model for the external flow. Such VII approach can model the effects of mild forms of boundary layer flow separation. A 3D integral boundary layer (IBL) method is recommended as it keeps the problem, like the panel method, restricted to the surface of the blades and the wake surfaces. For wind turbine applications, unsteady flow and the effects of rotation on the development of the boundary layer should be taken into account (see [36],[83]-[86],[103],[105]).

\section{Fluid-structure interaction}

The relative flexibility of wind turbine blades and the unsteady character of the aerodynamic loads make that the interaction between aerodynamic forces and structural dynamics must be taken into account to predict the behavior of a real wind turbine. This problem is commonly referred to as Fluid-Structure Interaction (FSI).

The unsteady aerodynamic loads causes the wind turbine to react through deflection, deformation, and acceleration of the structural components. The reaction of the structure in turn alters the aerodynamic forces on the configuration (see [29],[55]). A wind turbine control algorithm ideally avoids a positive feedback loop between fluid and structure and keeps the dynamic loads within bounds and reduces fatigue damage of the wind turbine structure.

Ideally, when the structure is represented by a surface model, it is possible to account in the panel method approach for the effects of local deformations and buckling of the rotor blade. Using a combination of panel method and IBL method, such FSI approach is much less cumbersome than an approach based on solving the Navier-Stokes equations on a volumetric discretization of the $3 \mathrm{D}$ computational domain [7]. 


\section{References}

[1] J.D. Anderson. Fundamentals of Aerodynamics. McGraw-Hill, fourth edition, 2006.

[2] Anon. Kyoto Protocol to the United Nations Framework Convention on Climate Change. Report, United Nations, 1998.

[3] Anon. Adoption of the Paris Agreement. Report, United Nations, 2015.

[4] Anon. Nationale Energieverkenning 2015. Report, Centraal Bureau voor de Statistiek, 2015.

[5] R. Aris. Vectors, Tensors, and the Basic Equations of Fluid Mechanics. Dover Publications, 1989.

[6] G.K. Batchelor. An Introduction to Fluid Dynamics. Cambridge University Press, 1967.

[7] Y. Bazilevs, K. Takizawa, and T.E. Tezduyar. Computational FluidStructure Interaction. Methods and Applications. John Wiley \& Sons Ltd, 2013.

[8] P.G. Bellamy-Knights, M.G. Benson, J.H. Gerrard, and I. Gladwell. Convergence properties of panel methods. Computer Methods in Applied Mechanics and Engineering, 76(2):171-178, 1989.

[9] J-P. Berrut and L.N. Trefethen. Barycentric Lagrange Interpolation. SIAM Review, 46(3):501-517, 2004.

[10] A. Betz. Wind Energie und ihre Ausnutzung durch Windmühlen. Vandenhoeck \& Ruprecht, 1926.

[11] A. Betz and L. Prandtl. Schraubenpropeller mit Geringstem Energieverlust. Nachrichten von der Gesellschaft der Wissenscaften zu Göttingen, 1919.

[12] H.A. Bijleveld. Application of Quasi-Simultaneous interaction method for the determination of the aerodynamic forces on wind turbine blades. PhD thesis, Rijksuniversiteit Groningen, 2012. 
[13] H.A. Bijleveld and A.E.P. Veldman. RotorFlow: A quasisimultaneous interaction for the prediction of aerodynamic flow over wind turbine blades. In The Science of Making Torque from Wind, 2010.

[14] L.M.M. Boermans and A. van Garrel. Design and Windtunnel Test Results of a Flapped Laminar Flow Airfoil for High-Performance Sailplane Applications. In ICAS 1994 Anaheim, pages 1241-1247, 1994.

[15] K. Boorsma and J.G. Schepers. New MEXICO experiment. Preliminary overview with initial validation. ECN-E--14-048, Energy research Centre of the Netherlands, 2014.

[16] S. Börm. $\mathcal{H}^{2}$-matrices - Multilevel methods for the approximation of integral operators. Preprint 7, Max-Planck-Institut für Mathematik in den Naturwissenschaften, Leipzig, 2003.

[17] S. Börm. Efficient Numerical Methods for Non-local Operators. $\mathcal{H}^{2}$ Matrix Compression, Algorithms, and Analysis. European Mathematical Society, 2010.

[18] J. Bos. Frictional Heating of Tribological Contacts. PhD thesis, University of Twente, 1995.

[19] A. Brandt. Guide to Multigrid Development. In Multigrid Methods. Proceedings of the Conference Held at Köln-Porz, November 23-27, 1981, pages 220-312, 1982.

[20] A. Brandt. Multilevel computations: Review and recent developments. In Proceedings of the Third Copper Mountain Conference on Multigrid Methods, Copper Mountain, Colorado, April 1987, pages 35-62, 1988.

[21] A. Brandt. Multilevel computations of integral transforms and particle interactions with oscillatory kernels. Computer Physics Communications, 1991.

[22] A. Brandt. Unpublished letter to S. Osher, V. Rokhlin, L. Greengard, Ami Harten, 1992.

[23] A. Brandt and A.A. Lubrecht. Multilevel Matrix Multiplications and Fast Solution of Integral Equations. Journal of Computational Physics, 90:348-370, 1990. 
[24] R.L. Carmichael and L.L. Erickson. PAN AIR - A Higher Order Panel Method for Predicting Subsonic or Supersonic Linear Potential Flows About Arbitrary Configurations. AIAA Paper 81-1255, AIAA, June 1981.

[25] A.H.-D Cheng and D.T. Cheng. Heritage and early history of the boundary element method. Engineering Analysis with Boundary Elements, 29:268-302, 2005.

[26] G.H. Cottet and P.D. Koumoutsakos. Vortex Methods: Theory and Practice. Cambridge University Press, 2000.

[27] W. Dobber. Cornelis Corneliszoon van Uitgeest en zijn bijdragen aan de eerste moderne economie van Europa. Report, Stichting Cornelis Corneliszoon van Uitgeest, 2014.

[28] J. Dölz, H. Harbrecht, and M. Peters. An interpolation-based fast multipole method for higher-order boundary elements on parametric surfaces. International Journal for Numerical Methods in Engineering, pages $\mathrm{n} / \mathrm{a}-\mathrm{n} / \mathrm{a}, 2016$. nme.5274.

[29] E.H. Dowell. A Modern Course in Aeroelasticity. Fifth Revised and Enlarged Edition, Vol. 217 of Solid Mechanics and Its Applications. Springer, 2015.

[30] M. Drela. XFOIL. http://web.mit.edu/drela/Public/web/xfoil/.

[31] M. Drela. XFOIL: An Analysis and Design System for Low Reynolds Number Airfoils. In T.J. Mueller, editor, Low Reynolds Number Aerodynamics, Vol. 54 of Lecture Notes in Engineering, pages 1-12. Springer-Verlag, New York, 1989.

[32] M. Drela. Three-Dimensional Integral Boundary Layer Formulation for General Configurations. AIAA Paper 2013-2437, AIAA, 2013.

[33] W.F. Durand, editor. Aerodynamic Theory, Vol. 1. Dover Publications, 1934.

[34] I.D. Faux and Pratt M.J. Computational Geometry for Design and Manufacture. Ellis Horwood Ltd, 1979.

[35] A. van Garrel. Development of a Wind Turbine Aerodynamics Simulation Module. ECN-C--03-079, Energy research Centre of the Netherlands, 2003. 
[36] A. van Garrel. Integral Boundary Layer Methods for Wind Turbine Aerodynamics. ECN-C--04-004, Energy research Centre of the Netherlands, 2004.

[37] A. van Garrel. Development of a Wind Turbine Rotor Flow Panel Method. ECN-E--11-071, Energy research Centre of the Netherlands, 2011.

[38] T.J. Garrett. Are there basic physical constraints on future anthropogenic emissions of carbon dioxide? Climatic Change, 104(3):437455, 2011.

[39] K. Giebermann. Multilevel Approximation of Boundary Integral Operators. Computing, 67:183-207, 2001.

[40] G. Green. An Essay on the Application of Mathematical Analysis to the Theories of Electricity and Magnetism, 1828.

[41] L. Greengard. The Rapid Evaluation of Potential Fields in Particle Systems. PhD thesis, Massachusetts Institute of Technology, 1988.

[42] L. Greengard and V. Rokhlin. A Fast Algorithm for Particle Simulations. Journal of Computational Physics, 73(2):325-348, 1987.

[43] L. Greengard and V. Rokhlin. A new version of the Fast Multipole Method for the Laplace equation in three dimensions. Acta Numerica, 6:229-269, 1997.

[44] D.A. Grier. When Computers Were Human. Princeton University Press, Princeton, USA, 2005.

[45] W. Hackbusch. A sparse matrix arithmetic based on $\mathcal{H}$-matrices. Part I: Introduction to $\mathcal{H}$-matrices. Computing, 62:89-108, 1999.

[46] W. Hackbusch. Panel Clustering Techniques and Hierarchical Matrices for BEM and FEM. Preprint 71, Max-Planck-Institut für Mathematik in den Naturwissenschaften, Leipzig, 2003.

[47] W. Hackbusch. Hierarchical Matrices: Algorithms and Analysis, Vol. 49 of Springer Series in Computational Mathematics. Springer, Berlin, Germany, 2015.

[48] W. Hackbusch and S. Börm. $\mathcal{H}^{2}$-matrix approximation of integral operators by interpolation. Applied Numerical Mathematics, 43:129$143,2002$. 
[49] W. Hackbusch and Z.P. Nowak. On the Fast Matrix Multiplication in the Boundary Element Method by Panel Clustering. Numerische Mathematik, 54:463-491, 1989.

[50] J.C. Heinz. Partitioned Fluid-Structure Interaction for Full Rotor Simulations using CFD. PhD thesis, Technical University of Denmark, 2013.

[51] I. Hérnandez Ramírez. Multilevel Multi-Integration Algorithm for Acoustics. PhD thesis, University of Twente, 2005.

[52] J.L. Hess. Calculation of Potential Flow About Arbitrary ThreeDimensional Lifting Bodies. MDC J5679-01, Douglas Aircraft Company, 1972.

[53] J.L. Hess and A.M.O. Smith. Calculation of non-lifting potential flow about arbitrary three-dimensional bodies. ES 40622, Douglas Aircraft Division, 1962.

[54] J.L. Hess and A.M.O. Smith. Calculation of potential flow about arbitrary bodies. Progress in Aerospace Sciences, 8:1-138, 1967.

[55] D.H. Hodges and G.A. Pierce. Introduction to Structural Dynamics and Aeroelasticity. Second Edition. Cambridge University Press, 2011.

[56] H.W.M. Hoeijmakers. A Panel Method for the Determination of the Aerodynamic Characteristics of Complex Configurations in Linearized Subsonic or Supersonic Flow. Technical Report NLR TR 80124, National Aerospace Laboratory of the Netherlands, 1980.

[57] H.W.M. Hoeijmakers. Computational aerodynamics of ordered vortex flows. PhD thesis, Delft University of Technology, 1989.

[58] H.W.M. Hoeijmakers. Panel Methods for Aerodynamic Analysis and Design. In Special Course on Engineering Methods in Aerodynamic Analysis and Design of Aircraft, AGARD Report R-783, 1991.

[59] M.-C. Hsu and Y. Bazilevs. Fluid-structure interaction modeling of wind turbines: simulating the full machine. Computational Mechanics, 50:821-833, 2012.

[60] F.T. Johnson. A General Panel Method for the Analysis and Design of Arbitrary Configurations in Incompressible Flows. Technical Report CR-3079, NASA, 1980.

[61] S.H. Jongsma. On a Method for Simulation-Based Wind Turbine Blade Design. PhD thesis, University of Twente, 2014. 
[62] J. Katz and B. Maskew. Unsteady Low-Speed Aerodynamic Model for Complete Aircraft Configurations. Journal of Aircraft, 25(4):302-310, 1988.

[63] J. Katz and A. Plotkin. Low-Speed Aerodynamics: From Wing Theory to Panel Methods. Cambridge University Press, second edition, 2001.

[64] D.P. Keenan. Marine Propellers in Unsteady Flow. PhD thesis, Massachusetts Institute of Technology, 1989.

[65] J.C. Kok. Resolving the Dependence on Freestream Values for the $k-\omega$ Turbulence Model. AIAA Journal, 38(7):1292-1295, 2000.

[66] J.C. Kok. A high-order low-dispersion symmetry-preserving finitevolume method for compressible flow on curvilinear grids. Journal of Computational Physics, 228:6811-6832, 2009.

[67] G.A.M van Kuik, J.N. Sørensen, and V.L. Okulov. Rotor theories by Professor Joukowsky: Momentum theories. Progress in Aerospace Sciences, 73:1-18, 2015.

[68] Th.E. Labrujère, W. Loeve, and J.W. Slooff. An Approximate Method for the Calculation of the Pressure Distribution on Wing-Body Combinations. In Aerodynamic Interference, AGARD CP-71, 1970.

[69] H. Lamb. Hydrodynamics. Cambridge University Press, sixth edition, 1932.

[70] M.J. Lighthill. On Displacement Thickness. Journal of Fluid Mechanics, 4:383-392, 1958.

[71] R.C. Lock and B.R. Williams. Viscous-Inviscid Interactions in External Aerodynamics. Progress in Aerospace Sciences, 24:51-171, 1987.

[72] A.A. Lubrecht and E. Ioannides. A Fast Solution of the Dry Contact Problem and the Associated Sub-Surface Stress Field, Using Multilevel Techniques. Journal of Tribology, 113(1):128-133, 1991.

[73] B. Maskew. Prediction of Subsonic Aerodynamic Characteristics: A Case for Low Order Panel Methods. Journal of Aircraft, 19(2):157163, 1982.

[74] M. Messner, B. Bramas, O. Coulaud, and E. Darve. Optimized M2L Kernels for the Chebyshev Interpolation based Fast Multipole Method. CoRR, abs/1210.7292, 2012. 
[75] L.M. Milne-Thomson. Theoretical Hydrodynamics. MacMillan \& Co, fourth edition, 1962.

[76] J. Moore, J. Peraire, and M. Drela. Progress Towards an Arbitrarily High-Order, Unstructured, Free-Wake Panel Solver. AIAA Paper 2013-2946, AIAA, 2013.

[77] L. Morino and C.-C. Kuo. Subsonic Potential Aerodynamics for Complex Configurations: A General Theory. AIAA Journal, 12(2):191197, 1974.

[78] P.M. Morse and H. Feshbach. Methods of Theoretical Physics, Vol. I. McGraw-Hill, 1953.

[79] P.M. Morse and H. Feshbach. Methods of Theoretical Physics, Vol. II. McGraw-Hill, 1953.

[80] J.N. Newman. Distributions of sources and normal dipoles over a quadrilateral panel. Journal of Engineering Mathematics, 20(2):113126, 1986.

[81] V.L. Okulov, J.N. Sørensen, and D.H. Wood. The rotor theories by Professor Joukowsky: Vortex theories. Progress in Aerospace Sciences, 73:19-46, 2015.

[82] F.W.J. Olver, D.W. Lozier, R.F. Boisvert, and C.W. Clark. NIST Handbook of Mathematical Functions. Cambridge University Press, first edition, 2010.

[83] H. Özdemir. Development of a discontinuous Galerkin method for the unsteady integral boundary layer equations. In Euromech Fluid Mechanics Conference - 8, 2010.

[84] H. Özdemir and E.F. van den Boogaard. Solving the integral boundary layer equations with a discontinuous Galerkin method. In EWEA 2011, 2011.

[85] H. Özdemir, A. van Garrel, and H.A. Bijleveld. RotorFlow: Development of a discontinuous Galerkin method for the integral boundary layer equations. In The Science of Making Torque from Wind, 2010.

[86] H. Özdemir, A. van Garrel, A.K. Ravishankara, F. Passalaqua, and H.J. Seubers. Unsteady Interacting Boundary Layer Method. Paper (accepted), AIAA, Texas, USA, January 2017. 
[87] B. Oskam. Transonic Panel Method for the Full Potential Equation Applied to Multicomponent Airfoils. AIAA Journal, 23(9):1327-1334, 1985.

[88] B. Oskam. Asymptotic Convergence of Higher-Order Accurate Panel Methods. Journal of Aircraft, 23(2):126-130, 1986.

[89] S. ten Pas. CFD simulations of the MEXICO wind turbine. Validating ENSOLV for wind turbine flows. Master's thesis, University of Twente, 2016.

[90] J.R. Phillips and J.K. White. A Precorrected-FFT method for Electrostatic Analysis of Complicated 3-D Structures. IEEE Transactions on Computer-Aided Design of Integrated Circuits and Systems, 16(10):1059-1072, 1997.

[91] W.J. Piers and J.W. Slooff. Calculation of transonic flow by means of a shock-capturing field panel method. AIAA Paper 79-1459, AIAA, 1979 .

[92] R. Postma. Shell in de race voor windenergie op zee. NRC, 2016.

[93] N. Ramos-García. Unsteady Viscous-Inviscid Interaction Technique for Wind Turbine Airfoils. PhD thesis, Technical University of Denmark, 2011.

[94] N. Ramos-García, J.N. Sørensen, and W.Z. Shen. Three-dimensional viscous-inviscid coupling method for wind turbine computations. Wind Energy, 19(1):67-93, 2016.

[95] V. Rokhlin. Rapid Solution of Integral Equations of Classical Potential Theory. Journal of Computational Physics, 60:187-207, 1983.

[96] Y. Saad and M.H. Schultz. GMRES: A Generalized Minimal Residual Algorithm for Solving Nonsymmetric Linear Systems. SIAM J. Sci. Stat. Comput., 7(3):856-869, July 1986.

[97] P.G. Saffman. Vortex Dynamics. Cambridge Monographs on Mechanics and Applied Mathematics. Cambridge University Press, 1992.

[98] B. Sandak. Multiscale Fast Summation of Long-Range Charge and Dipolar Interactions. Journal of Computational Chemistry, 22(7):717731, 2001.

[99] J.G. Schepers. Engineering models in wind energy aerodynamics: Development, implementation and analysis using dedicated aerodynamic measurements. PhD thesis, Delft University of Technology, 2012. 
[100] J.G. Schepers, K. Boorsma, T. Cho, S. Gomez-Iradi, P. Schaffarczyk, A. Jeromin, W.Z. Shen, T. Lutz, K. Meister, B. Stoevesandt, S. Schreck, D. Micallef, R. Pereira, T. Sant, H.A. Madsen, and N.N. Sørensen. Final report of IEA Wind Task 29: Mexnext (Phase 1). ECN-E--12-004, Energy research Centre of the Netherlands, 2012.

[101] J.G. Schepers, K. Boorsma, S. Gomez-Iradi, P. Schaffarczyk, H.A. Madsen, N.N. Sørensen, W.Z. Shen, T. Lutz, C. Schulz, I. Herraez, and S. Schreck. Final report of IEA Wind Task 29: Mexnext (Phase 2). ECN-E--14-060, Energy research Centre of the Netherlands, 2014.

[102] J.G. Schepers and H. Snel. Model Experiments in Controlled Conditions. ECN-E--07-042, Energy research Centre of the Netherlands, 2007.

[103] H. Snel, R. Houwink, and J. Bosschers. Sectional Prediction of Lift Coefficients on Rotating Wind Turbine Blades in Stall. ECN-C--93052, Energy research Centre of the Netherlands, 1994.

[104] H. Snel, J.G. Schepers, and N.B. Siccama. MEXICO Project: The Database and Results of Data Processing and Interpretation. AIAA Paper 2009-1217, AIAA, January 2009.

[105] J.N. Sørensen. Prediction of Three-Dimensional Stall on Wind Turbine Blade using Three-Level, Viscous-Inviscid Interaction Model. In EWEC '86, European Wind Energy Association Conference and Exhibition, pages 429-435, 1986.

[106] J.N. Sørensen. General Momentum Theory for Horizontal Axis Wind Turbines. Springer, 2016.

[107] A.E.P. Veldman. The Calculation of Incompressible Boundary Layers with Strong Viscous-Inviscid Interaction. In Computation of Viscous/Inviscid Interactions, AGARD CP-291, 1980.

[108] A.E.P. Veldman. New, Quasi-Simultaneous Method to Calculate Interacting Boundary Layers. AIAA Journal, 19(1):79-86, 1981.

[109] A.E.P. Veldman. A simple interaction law for viscous-inviscid interaction. Journal of Engineering Mathematics, 65(4):367-383, 2009.

[110] C.H. Venner. Multilevel Solution of the EHL Line and Point Contact Problems. PhD thesis, University of Twente, 1991. 
[111] C.H. Venner and A.A. Lubrecht. Multilevel Methods in Lubrication, Vol. 37 of Tribology Series. Elsevier Science, 2000.

[112] A.J.J. Verhoeff. Aerodynamics of Wind Turbine Rotors. PhD thesis, University of Twente, 2005.

[113] H.A. van der Vorst. Iterative Krylov methods for large linear systems. Cambridge Monographs on Applied and Computational Mathematics. Cambridge University Press, Cambridge, UK, New York, 2003.

[114] H. de Vries. On Synthetic Jet Actuation for Aerodynamic Load Control. PhD thesis, University of Twente, 2013.

[115] J. de Vries and A. van der Woude. Nederland 1500-1815. De eerste ronde van moderne economische groei. Uitgeverij Balans, 2005.

[116] O. de Vries. Fluid Dynamic Aspects of Wind Energy Conversion, Vol. AGARD-AG-243. AGARD, 1978.

[117] A.J. van der Wees and J. van Muijden. A Robust Quasi-Simultaneous Interaction Method for a Full Potential Flow with a Boundary Layer with Application to Wing-Body Configurations. In Fifth Symposium on Numerical and Physical Aspects of Aerodynamic Flows, January 1992.

[118] Y.H. Wijnant. Contact Dynamics in the field of Elastohydrodynamic Lubrication. PhD thesis, University of Twente, 1998.

[119] D.J. Willis. An Unsteady, Accelerated, High Order Panel Method with Vortex Particle Wakes. PhD thesis, Massachusetts Institute of Technology, 2006.

[120] D.J. Willis, J. Peraire, and J.K. White. A combined pFFT-multipole tree code, unsteady panel method with vortex particle wakes. International Journal for Numerical Methods in Fluids, 53:1399-1422, 2007. 


\section{A Mathematical Compendium}

This chapter contains a collection of some mathematical formulas useful in the present context. For formulas in index notation, summation over repeated indices is assumed.

Delta function: The Dirac delta function has the property that

$$
\delta(x)=0 \quad \text { for } x \neq 0,
$$

and that

$$
\int f(x) \delta(x-a) d x=f(a),
$$

provided that the point $x=a$ is included in the integration interval. In three-dimensional Cartesian space the delta function is defined by

$$
\delta\left(\vec{r}-\vec{r}_{0}\right)=\delta\left(x-x_{0}\right) \delta\left(y-y_{0}\right) \delta\left(z-z_{0}\right),
$$

and enables the expression of a discrete quantity at $\vec{r}_{0}$ in a volume integral as if it were a continuous distribution.

Divergence Theorem: The Divergence Theorem is Gauss' theorem for a vector function $\vec{b}$ and relates the volume and surface integrals of the vector field in a volume $V$ enclosed by the surface $\partial V$ by:

$$
\iiint_{V}(\nabla \cdot \vec{b}) d V=\iint_{\partial V}(\vec{b} \cdot \bar{n}) d S .
$$

Gauss' Theorem: Gauss' theorem gives a relation between the volume and surface integrals of a continuously differentiable (i.e. $C^{1}$ functions with continuous derivative) arbitrary tensor function $T_{i j}$ over a volume $V$ enclosed by piecewise smooth boundary $\partial V$ with outward unit normal vector $\bar{n}$. The tensor $T_{j k}$ may be a scalar, vector or tensor function of any rank. In index notation Gauss' theorem reads:

$$
\iiint_{V} \partial_{i}\left(T_{j k}\right) d V=\iint_{\partial V} n_{i} T_{j k} d S .
$$

Some special forms of Gauss' theorem are obtained when specific choices are substituted for tensor $T_{j k}$. Substituting vector field $b_{i}$ 
for $T_{j k}$ for example gives the Divergence Theorem and when scalar field $\phi$ is substituted for $T_{j k}$ we arrive at the Gradient Theorem.

Substitution of $\epsilon_{k i j} b_{j}$ for tensor $T_{j k}$ gives

$$
\iiint_{V}(\nabla \times \vec{b}) d V=\iint_{\partial V}(\bar{n} \times \vec{b}) d S
$$

Gradient Theorem: The Gradient Theorem is Gauss' theorem for a scalar function $\phi$ and relates the volume and surface integrals of the scalar field over a volume $V$ enclosed by the surface $\partial V$ by:

$$
\iiint_{V}(\nabla \phi) d V=\iint_{\partial V} \phi \bar{n} d S
$$

Green's identities: Green's first identity is obtained when $\left(\psi \partial_{i} \phi\right)$ is substituted into Gauss' theorem for $T_{j k}$, where $\psi$ and $\phi$ are once and twice continuously differentiable scalar functions respectively:

$$
\iiint_{V} \psi \nabla^{2} \phi d V=\iint_{\partial V} \psi \frac{\partial \phi}{\partial n} d S-\iiint_{V} \nabla \phi \cdot \nabla \psi d V
$$

where $\partial \phi / \partial n=\nabla \phi \cdot \bar{n}$ denotes the derivative in the direction of the outward normal and $\nabla^{2}=\nabla \cdot \nabla$ is the Laplacian operator.

Green's second identity is obtained from the first identity by interchanging the role of $\psi$ and $\phi$ and subtract the resulting equations. Both $\psi$ and $\phi$ are assumed to be twice continuously differentiable scalar functions. Green's second identity reads:

$$
\iiint_{V}\left(\psi \nabla^{2} \phi-\phi \nabla^{2} \psi\right) d V=\iint_{\partial V}\left(\psi \frac{\partial \phi}{\partial n}-\phi \frac{\partial \psi}{\partial n}\right) d S .
$$

Stokes' Theorem: Stokes' theorem relates the line integral of a smooth vector field $\vec{b}$ over a closed curve $\partial S$ to the surface integral of $\vec{b}$ over an open surface $S$ bounded by curve $\partial S$. The orientations of unit tangent vector $\bar{\tau}$ to curve $\partial S$ and the unit normal vector $\bar{n}$ to surface $S$ are related through the right-hand rule.

Stokes' theorem reads:

$$
\iint_{S}(\nabla \times \vec{b}) \cdot \bar{n} d S=\int_{\partial S} \vec{b} \cdot \bar{\tau} d s
$$




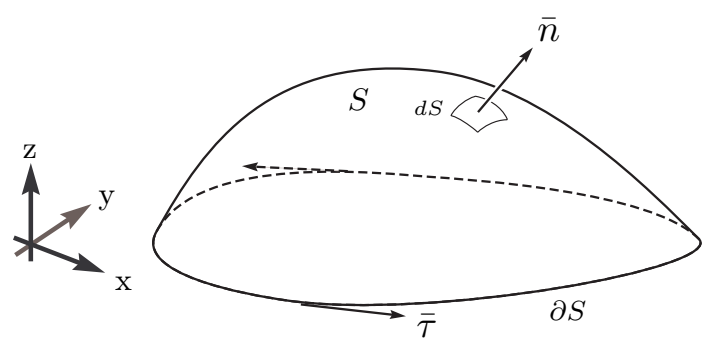

Other forms are

$$
\iint_{S}(\bar{n} \times \nabla f) d S=\int_{\partial S} f \bar{\tau} d s
$$

and

$$
\iint_{S}(\bar{n} \times \nabla) \times \vec{b} d S=-\int_{\partial S} \vec{b} \times \bar{\tau} d s .
$$

Substantial derivative: The substantial derivative, also know as material derivative, is the time rate of change following an infinitesimal impermeable control volume, a so-called fluid element, moving with fluid velocity $\vec{u}$. It can be split into the local and the convective derivative:

$$
\frac{\mathrm{D}()}{\mathrm{D} t}=\frac{\partial()}{\partial t}+\vec{u} \cdot \nabla() .
$$




\section{Vector identities:}

$$
\begin{aligned}
\vec{a} \cdot(\vec{b} \times \vec{c}) & =\vec{b} \cdot(\vec{c} \times \vec{a})=\vec{c} \cdot(\vec{a} \times \vec{b}) \\
\vec{a} \times(\vec{b} \times \vec{c}) & =\vec{b}(\vec{a} \cdot \vec{c})-\vec{c}(\vec{a} \cdot \vec{b}) \\
(\vec{a} \times \vec{b}) \times \vec{c} & =\vec{b}(\vec{a} \cdot \vec{c})-\vec{a}(\vec{b} \cdot \vec{c}) \\
(\vec{a} \times \vec{b}) \cdot(\vec{c} \times \vec{d}) & =(\vec{a} \cdot \vec{c})(\vec{b} \cdot \vec{d})-(\vec{a} \cdot \vec{d})(\vec{b} \cdot \vec{c}) \\
(\vec{a} \times \vec{b}) \times(\vec{c} \times \vec{d}) & =\vec{c}(\vec{a} \cdot(\vec{b} \times \vec{d}))-\vec{d}(\vec{a} \cdot(\vec{b} \times \vec{c})) \\
& =\vec{b}(\vec{a} \cdot(\vec{c} \times \vec{d})-\vec{a}(\vec{b} \cdot(\vec{c} \times \vec{d})) \\
\vec{x} & =\frac{\vec{x} \cdot(\vec{b} \times \vec{c})}{\vec{a} \cdot(\vec{b} \times \vec{c})} \overrightarrow{\vec{x} \cdot(\vec{c} \times \vec{a})} \vec{b}+\frac{\vec{x} \cdot(\vec{a} \times \vec{b})}{\vec{a} \cdot(\vec{b} \times \vec{c})} \vec{c} \\
\nabla(f g) & =f(\nabla g)+g(\nabla f) \\
\nabla(\vec{a} \cdot \vec{b}) & =\vec{a} \times(\nabla \times \vec{b})+\vec{b} \times(\nabla \times \vec{a})+(\vec{a} \cdot \nabla) \vec{b}+(\vec{b} \cdot \nabla) \vec{a} \\
\nabla \cdot(f \vec{a}) & =f(\nabla \cdot \vec{a})+\vec{a} \cdot(\nabla f) \\
\nabla \cdot(\vec{a} \times \vec{b}) & =\vec{b} \cdot(\nabla \times \vec{a})-\vec{a} \cdot(\nabla \times \vec{b}) \\
\nabla \times(\vec{a} \times \vec{b}) & =(\vec{b} \cdot \nabla) \vec{a}-(\vec{a} \cdot \nabla) \vec{b}+\vec{a}(\nabla \cdot \vec{b})-\vec{b}(\nabla \cdot \vec{a}) \\
\nabla \times(f \vec{a}) & =f(\nabla \times \vec{a})+\nabla f \times \vec{a} \\
\nabla \times(\nabla \times \vec{a}) & =\nabla(\nabla \cdot \vec{a})-(\nabla \cdot \nabla) \vec{a} \\
\vec{a} \cdot((\vec{b} \times \nabla) \times \vec{c}) & =((\vec{a} \cdot \nabla) \vec{c}) \cdot \vec{b}-(\vec{a} \cdot \vec{b})(\nabla \cdot \vec{c}) \\
\nabla \cdot(\nabla \times \vec{a}) & =0 \\
\nabla \cdot(\nabla f \times \nabla g) & =0 \\
\nabla \times \nabla f & =\overrightarrow{0}
\end{aligned}
$$

Define a 3D Cartesian displacement vector and its length as $\vec{r}$ and $r=|\vec{r}|$. Then

$$
\begin{aligned}
\nabla r^{n} & =n r^{n-2} \vec{r} \\
(\nabla \cdot \nabla) r^{n} & =n(n+1) r^{n-2} \\
\nabla \cdot\left(r^{n} \vec{r}\right) & =(n+3) r^{n} \\
\nabla \times\left(r^{n} \vec{r}\right) & =\overrightarrow{0} \\
\nabla \cdot \vec{r} & =3 \\
\nabla \times \vec{r} & =\overrightarrow{0}
\end{aligned}
$$




\section{B Conservation Laws}

In this chapter the derivation of the conservation laws for mass, momentum, and energy is given for reasons of completeness. They can also be found in most textbooks on fluid mechanics. A clear treatise is, for example, given in reference [1].

\section{B.1 Mass Conservation}

Let us consider an arbitrary control volume $V$ fixed in space. Fluid is freely flowing with velocity $\vec{u}(\vec{x}, t)$ through its bounding surface $\partial V$ with unit normal vector $\bar{n}(\vec{x})$ pointing outward as shown in Figure B.1. The equation for mass conservation, also known as the continuity equation, expresses that an increase in mass in volume $V$ can only come from a net mass flow through its bounding surface $\partial V$

$$
\frac{\mathrm{d}}{\mathrm{d} t}(\text { Mass in } V)=\text { Net mass inflow per unit time. }
$$

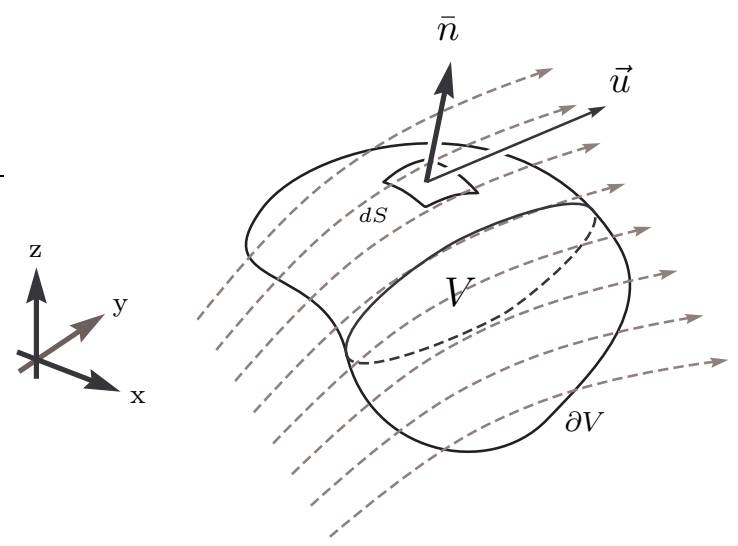

Figure B.1: A control volume $V$ fixed in space with fluid freely flowing through its bounding surface $\partial V$.

Considering that $\vec{u} \cdot \bar{n} d S$ is the volume that flows out of area $d S$ per unit time, we can write for the net mass flowing through the surface into control 
volume $V$ per unit time:

$$
\text { Mass inflow }=-\iint_{\partial V} \rho \vec{u} \cdot \bar{n} d S,
$$

where $\rho(\vec{x}, t)$ is the mass density of the fluid and the minus sign stems from the definition of the normal vector as positive when pointing outward. The integral form of the equation for mass conservation can now be written as

$$
\frac{\mathrm{d}}{\mathrm{d} t} \iiint_{V} \rho d V+\iint_{\partial V} \rho \vec{u} \cdot \bar{n} d S=0 .
$$

We can use the Divergence Theorem to transform the surface integral into a volume integral, provided we have a continuously differentiable vector field $\rho \vec{u}:$

$$
\frac{\mathrm{d}}{\mathrm{d} t} \iiint_{V} \rho d V+\iiint_{V} \nabla \cdot(\rho \vec{u}) d V=0,
$$

which can be written as

$$
\iiint_{V}\left(\frac{\partial \rho}{\partial t}+\nabla \cdot(\rho \vec{u})\right) d V=0
$$

where we used that the control volume is fixed in space to shift the time derivative to the integrand. Because this equation is valid for arbitrary control volumes, the integrand has to be zero for any point within $V$, and we arrive at the differential form of the continuity equation

$$
\frac{\partial \rho}{\partial t}+\nabla \cdot(\rho \vec{u})=0
$$

for all points in $V$, which can also be written as

$$
\nabla \cdot \vec{u}=-\frac{1}{\rho} \frac{\mathrm{D} \rho}{\mathrm{D} t} .
$$

For incompressible flow the mass density $\rho$ is constant and equation (B.2) reduces to a form without time derivative term

$$
\nabla \cdot \vec{u}=0 .
$$

If we assume irrotational flow $\nabla \times \vec{u}=\overrightarrow{0}$. Then we can write the velocity vector as the gradient of a scalar function $\Phi(\vec{x}, t)$, that is

$$
\vec{u}=\nabla \Phi \text {. }
$$

Substitution of this expression for the velocity vector into the continuity equation for incompressible flow (B.4) results in the Laplace equation for the yet unknown velocity potential $\Phi$ :

$$
\nabla \cdot \nabla \Phi=\frac{\partial^{2} \Phi}{\partial x^{2}}+\frac{\partial^{2} \Phi}{\partial y^{2}}+\frac{\partial^{2} \Phi}{\partial z^{2}}=0
$$




\section{B.2 Momentum Conservation}

Momentum conservation is expressed by Newton's second law of motion that states that, viewed from an inertial frame of reference, the time rate of change of the linear momentum of a particle is proportional to the net force excerted on it:

$$
\frac{\mathrm{d}}{\mathrm{d} t}(m \vec{u})=\vec{F}
$$

where $m$ is the mass of the particle and $\vec{u}$ its velocity.

Using our fixed control volume from Figure B.1, the equation for momentum conservation expresses that an increase in momentum in volume $V$ can only come from a net momentum flow through its surface or from a force exerted on the fluid. The equation for conservation of linear momentum can be written symbolically as

$$
\frac{\mathrm{d}}{\mathrm{d} t}(\text { Momentum in } V)=\text { Net momentum inflow per unit time }+\vec{F}
$$

The net momentum flowing through the surface into the control volume per unit time is equal to the net mass flow times the momentum per unit mass. The momentum per unit mass is just the velocity, so that

$$
\text { Momentum inflow }=-\iint_{\partial V} \rho \vec{u}(\vec{u} \cdot \bar{n}) d S,
$$

where the minus sign stems from the definition of the unit surface normal as positive when pointing outward. The momentum equation can now be written in integral conservation form as

$$
\frac{\mathrm{d}}{\mathrm{d} t} \iiint_{V} \rho \vec{u} d V+\iint_{\partial V} \rho \vec{u}(\vec{u} \cdot \bar{n}) d S=\vec{F},
$$

The force $\vec{F}$ acting on the fluid can be split into body forces that act upon the mass inside the control volume, and forces that act on the surface bounding the volume. Body forces are of the form

$$
\vec{F}_{b o d y}=\iiint_{V} \rho \vec{f} d V
$$

where $\vec{f}$ is defined as the force per unit mass. An example of such a body force is the force caused by gravity. With the gravitational acceleration vector denoted by $\vec{g}$ we can write this body force as

$$
\vec{F}_{\text {gravity }}=\iiint_{V} \rho \vec{g} d V
$$


The forces that act on the surface of the control volume can be decomposed into a force due to the static pressure $p$ acting on surface $\partial V$ in normal direction

$$
\vec{F}_{\text {pressure }}=-\iint_{\partial V} p \bar{n} d S,
$$

and a force due to the viscosity of the fluid, that is, the viscous stress $\vec{\tau} \equiv \overline{\bar{\tau}} \cdot \bar{n}$ :

$$
\vec{F}_{\text {viscous }}=\iint_{\partial V} \vec{\tau} d S=\iint_{\partial V} \overline{\bar{\tau}} \cdot \bar{n} d S
$$

Including the forces due to gravity (B.9), pressure (B.10) and viscous stress (B.11) in the momentum conservation equations (B.7) results in

$$
\frac{\mathrm{d}}{\mathrm{d} t} \iiint_{V} \rho \vec{u} d V+\iint_{\partial V} \rho \vec{u}(\vec{u} \cdot \bar{n}) d S=\iiint_{V} \rho \vec{g} d V-\iint_{\partial V} p \bar{n} d S+\iint_{\partial V} \overline{\bar{\tau}} \cdot \bar{n} d S .
$$

We can use the Divergence Theorem and Gradient Theorem to transform the surface integrals in equation (B.12) into volume integrals, provided we have a continuously differentiable tensor field $\rho \vec{u} \vec{u}$, pressure field $p$, and stress tensor field $\overline{\bar{\tau}}$, giving

$$
\iiint_{V}\left(\frac{\partial(\rho \vec{u})}{\partial t}+\nabla \cdot(\rho \vec{u} \vec{u})+\nabla p-\rho \vec{g}-\nabla \cdot \overline{\bar{\tau}}\right) d V=\overrightarrow{0},
$$

where we used that the control volume is fixed and we are allowed to shift the time derivative to the integrand. Because this equation is valid for arbitrary control volumes, the integrand on the left hand side has to balance the right-hand side everywhere within $V$, and we arrive at the differential form of the momentum equations:

$$
\frac{\partial(\rho \vec{u})}{\partial t}+\nabla \cdot(\rho \vec{u} \vec{u})+\nabla p-\rho \vec{g}-\nabla \cdot \overline{\bar{\tau}}=\overrightarrow{0},
$$

for all points within $V$.

For Newtonian fluids the viscous stresses are considered to be proportional to velocity gradients through the constitutive relation

$$
\overline{\bar{\tau}}=\mu\left[\nabla \vec{u}+(\nabla \vec{u})^{\mathrm{T}}-\frac{2}{3}(\nabla \cdot \vec{u}) \overline{\bar{I}}\right],
$$

where the dynamic viscosity coefficient $\mu$ is in general a function of temperature. 
This result can be simplified considerably if we assume the flow to be inviscid and incompressible. The constant mass density and constant gravitational acceleration vector $\vec{g}$ allow us to combine the pressure and gravitational terms in the momentum equations (B.13). Writing $\rho \vec{g}=\nabla(\rho \vec{g} \cdot \vec{x})$ and using the continuity equation (B.4) for incompressible flows, we arrive at the differential form of the momentum equations for inviscid, incompressible flows:

$$
\rho_{\infty} \frac{\partial \vec{u}}{\partial t}+\rho_{\infty}(\vec{u} \cdot \nabla) \vec{u}+\nabla\left(p-\rho_{\infty} \vec{g} \cdot \vec{x}\right)=\overrightarrow{0} .
$$

Using one of the vector identities from appendix A, the second term in above equation can be witten as

$$
(\vec{u} \cdot \nabla) \vec{u}=\nabla\left(\frac{1}{2} \vec{u} \cdot \vec{u}\right)-\vec{u} \times(\nabla \times \vec{u}) .
$$

Assuming steady flow, the substitution of the vector identity (B.16) in the momentum equations (B.15) for inviscid, incompressible flow gives

$$
\nabla\left(\frac{1}{2} \vec{u} \cdot \vec{u}+\frac{p}{\rho_{\infty}}-\vec{g} \cdot \vec{x}\right)-\vec{u} \times(\nabla \times \vec{u})=\overrightarrow{0} .
$$

Taking the inner product of this equation with the velocity vector $\vec{u}$ results in

$$
\vec{u} \cdot \nabla\left(\frac{1}{2} \vec{u} \cdot \vec{u}+\frac{p}{\rho_{\infty}}-\vec{g} \cdot \vec{x}\right)=0,
$$

which gives us the Bernoulli equation for steady, incompressible, inviscid, and rotational flow that is valid in every point on a streamline through $\vec{x}_{0}$ :

$$
\frac{1}{2} \vec{u} \cdot \vec{u}+\frac{p}{\rho_{\infty}}-\vec{g} \cdot \vec{x}=c\left(\vec{x}_{0}\right) .
$$

Let us now start again with the momentum equations for unsteady, inviscid, incompressible flow (B.15), and assume an irrotational velocity field, that is, $\nabla \times \vec{u}=\overrightarrow{0}$. We can then introduce $\vec{u}=\nabla \Phi$, and with the use of vector identity (B.16) arrive at

$$
\nabla\left(\rho_{\infty} \frac{\partial \Phi}{\partial t}+\frac{1}{2} \rho_{\infty} \nabla \Phi \cdot \nabla \Phi+p-\rho_{\infty} \vec{g} \cdot \vec{x}\right)=\overrightarrow{0},
$$

which gives the Bernoulli equation for unsteady, incompressible, potential flow:

$$
\frac{\partial \Phi}{\partial t}+\frac{1}{2} \nabla \Phi \cdot \nabla \Phi+\frac{p}{\rho_{\infty}}-\vec{g} \cdot \vec{x}=C(t),
$$

everywhere in the flow domain. 


\section{B.3 Energy Conservation}

The equation for conservation of energy is based on the first law of thermodynamics that states that an increase in total energy in a volume $V$ per unit time can only come from the energy added by heat per unit time or from the work per unit time done by forces. Using the fixed control volume from Figure B.1, the equation for conservation of energy for fluid flows can be expressed symbolically by

$\frac{\mathrm{d}}{\mathrm{d} t}($ Energy in $V)=$ Net energy inflow per unit time $+\dot{\text { Heat }}+\dot{\text { Work. }}$

Let $E(\vec{x}, t)$ be the total specific energy, that is the total energy per unit mass, and let $e(\vec{x}, t)$ be the internal specific energy that comes from random molecular motion. The total specific energy is the sum of the internal specific energy and the kinetic specific energy that is associated with the bulk velocity $\vec{u}(\vec{x}, t)$, that is

$$
E \stackrel{\text { def }}{=} e+\frac{1}{2} \vec{u} \cdot \vec{u}
$$

The total energy in volume $V$ is

$$
\text { Energy in } V=\iiint_{V} \rho E d V \text {. }
$$

For the net energy flowing through the surface $\partial V$ into the control volume per unit time we can write

$$
\text { Energy inflow }=-\iint_{\partial V} \rho E(\vec{u} \cdot \bar{n}) d S,
$$

where the minus sign stems from the definition of the unit surface normal as positive when pointing outward. The expression for energy conservation can now be written as

$$
\frac{\mathrm{d}}{\mathrm{d} t} \iiint_{V} \rho E d V+\iint_{\partial V} \rho E(\vec{u} \cdot \bar{n}) d S=\dot{\text { Heat }}+\text { Work. }
$$

The heat that is added to the volume can be split into volumetric heating that acts on the fluid inside the volume, and heat transfer at the surface that acts through thermal conduction at the surface bounding the control volume and is caused by gradients in the temperature distribution. Let $\dot{Q}(\vec{x}, t)$ denote the volumetric heat transfer in joule per unit volume per 
unit time, and let $\vec{q}$ be the conductive heat flux vector in joule per unit area per unit time, then

$$
\begin{aligned}
\text { Volumetric heating } & =\iiint_{V} \dot{Q} d V, \\
\text { Surface heat transfer } & =-\iint_{\partial V} \vec{q} \cdot \bar{n} d S .
\end{aligned}
$$

In general, for the work done per unit time by applying a force $\vec{f}(\vec{x}, t)$ to the fluid moving with velocity $\vec{u}(\vec{x}, t)$ we can write

$$
\dot{\mathrm{W}}_{\text {force }}=\vec{f} \cdot \vec{u}
$$

The forces can be split into volumetric forces that act upon the mass inside the volume, and forces that act via the surface of the volume, see Appendix B.2.

An example of a volumetric force is the gravitational force. With the gravitational acceleration vector denoted by $\vec{g}$ we can write the work done per unit time by this body force as

$$
\dot{\mathrm{W}}_{\text {gravity }}=\iiint_{V} \rho \vec{g} \cdot \vec{u} d V .
$$

The forces that act over the surface of the control volume can be decomposed into a force in normal direction due to the static pressure $p(\vec{x}, t)$ acting on surface $\partial V$ and a force due to the viscous stress. The work done by the static pressure per unit time is

$$
\dot{\mathrm{W}}_{\text {pressure }}=-\iint_{\partial V} p \vec{u} \cdot \bar{n} d S,
$$

and for the work done by viscous forces per unit time we have

$$
\dot{\mathrm{W}}_{\text {viscous }}=\iint_{\partial V} \vec{\tau} \cdot \vec{u} d S=\iint_{\partial V}(\overline{\bar{\tau}} \cdot \vec{u}) \cdot \bar{n} d S .
$$

Combining all contributions of heat and work in the expression for conservation of energy (B.20) results in the energy equation in integral conservation 
form:

$$
\begin{aligned}
\frac{\mathrm{d}}{\mathrm{d} t} \iiint_{V} \rho E d V+\iint_{\partial V} \rho E(\vec{u} \cdot \bar{n}) d S & =-\iint_{\partial V} p \vec{u} \cdot \bar{n} d S+\iint_{\partial V}(\overline{\bar{\tau}} \cdot \vec{u}) \cdot \bar{n} d S . \\
& +\iiint_{V} \dot{Q} d V-\iint_{\partial V} \vec{q} \cdot \bar{n} d S \\
& +\iiint_{V} \rho \vec{g} \cdot \vec{u} d V
\end{aligned}
$$

We can use the Divergence Theorem to convert the surface integrals to volume integrals, provided we have continuously differentiable vector fields $\rho E \vec{u}, p \vec{u}, \vec{q}$, and $\overline{\bar{\tau}} \cdot \vec{u}$, leading to

$$
\begin{aligned}
\iiint_{V} \frac{\partial(\rho E)}{\partial t} d V+\iiint_{V} \nabla \cdot(\rho E \vec{u}) d V & =-\iiint_{V} \nabla \cdot(p \vec{u}) d V+\iiint_{V} \nabla \cdot(\overline{\bar{\tau}} \cdot \vec{u}) d V \\
& +\iiint \dot{Q} d V-\iiint_{V} \nabla \cdot \vec{q} d V \\
& +\iiint_{V} \rho \vec{g} \cdot \vec{u} d V
\end{aligned}
$$

where we used that the control volume is fixed and we are allowed to shift the time derivative to the integrand. Because this equation is valid for arbitrary control volumes, the integrals have to balance each other everywhere in volume $V$ and consequently the energy equation can be written in differential form:

$$
\frac{\partial(\rho E)}{\partial t}+\nabla \cdot(\rho E \vec{u})+\nabla \cdot(p \vec{u})-\rho \vec{g} \cdot \vec{u}-\dot{Q}+\nabla \cdot \vec{q}-\nabla \cdot(\overline{\bar{\tau}} \cdot \vec{u})=0
$$

for a point inside $V$.

We can rewrite the above energy equation in terms of specific total enthalphy $H(\vec{x}, t)$ which is defined as

$$
H \stackrel{\text { def }}{=} E+\frac{p}{\rho}
$$

The substitution of the definition for total specific enthalphy in energy equation (B.28) gives a simpler equation for the conservation of energy that does not explicitly contain the term with the work done by the pressure:

$$
\frac{\partial(\rho H)}{\partial t}+\nabla \cdot(\rho H \vec{u})=\frac{\partial p}{\partial t}+\rho \vec{g} \cdot \vec{u}+\dot{Q}-\nabla \cdot \vec{q}+\nabla \cdot(\overline{\bar{\tau}} \cdot \vec{u}) .
$$


Expanding the term on the left hand side of the equation, and making use of the continuity equation (B.2), the non-conservation form of the equation for total enthalphy $H$ is obtained

$$
\rho \frac{\mathrm{D} H}{\mathrm{D} t}=\frac{\partial p}{\partial t}+\rho \vec{g} \cdot \vec{u}+\dot{Q}-\nabla \cdot \vec{q}+\nabla \cdot(\overline{\bar{\tau}} \cdot \vec{u}) .
$$

The set of conservation equations is supplemented with two constitutive relations, one for the viscous stress tensor $\overline{\bar{\tau}}(\vec{x}, t)$ and one for the heat flux vector $\vec{q}(\vec{x}, t)$. A model for the heat flux vector is given by Fourier's law

$$
\vec{q}=-\kappa \nabla T,
$$

where $\kappa$ is the thermal conductivity coefficient, and $T(\vec{x}, t)$ the temperature field.

The constitutive relation for the stress tensor was introduced in the derivation of the momentum equations in Appendix B.2 and is

$$
\overline{\bar{\tau}}=\mu\left[\nabla \vec{u}+(\nabla \vec{u})^{\mathrm{T}}-\frac{2}{3}(\nabla \cdot \vec{u}) \overline{\bar{I}}\right],
$$

with $\mu$ the dynamic viscosity coefficient that in general is a function of temperature $T$.

Two equations of state complement the set of equations: one expressing the relation between pressure $p$, mass density $\rho$, and temperature $T$, and one expression for e.g. the specific internal energy $e$. For a calorically perfect gas the two equations of state are the thermal equations of state

$$
p=\rho R T,
$$

with $R=c_{p}-c_{v}$ the specific gas constant which for air is approximately $R=287\left[\mathrm{~J} \cdot \mathrm{kg}^{-1} \cdot \mathrm{K}^{-1}\right]$, and the caloric equation of state

$$
e=c_{v} T,
$$

with $c_{v}$ the (constant) specific heat at constant volume.

Related to these two expressions and the definition of the specific enthalpy $h \stackrel{\text { def }}{=} e+\frac{p}{\rho}$ is the relation

$$
h=c_{p} T,
$$

with $c_{p}$ the (constant) specific heat at constant pressure. The ratio between the two specific heats is denoted by $\gamma=\frac{c_{p}}{c_{v}}$. For air within a certain range of conditions the heat capacity ratio is $\gamma=1.4$. 


\section{Helmholtz Decomposition}

Helmholtz' theorem of vector calculus states that a general continuously differentiable vector field in 3D space can be composed of a harmonic (i.e. irrotational and solenoidal) vector field $\vec{h}$, an irrotational vector field $\nabla \psi$, and a solenoidal vector field $\nabla \times \vec{\alpha}$ as

$$
\vec{a}=\vec{h}+\nabla \psi+\nabla \times \vec{\alpha},
$$

where $\vec{\alpha}$ itself is solenoidal, that is, $\nabla \cdot \vec{\alpha}=0$. Note that the harmonic part can also be written as the gradient of a scalar field and be combined with the second term (see for example Aris [5] and Saffman [97]). We have for $\vec{x} \in V$

$$
\begin{aligned}
\psi(\vec{x}) & =\frac{-1}{4 \pi} \iiint_{V} \frac{\nabla \cdot \vec{a}}{r} d V+\frac{1}{4 \pi} \iint_{\partial V} \frac{\vec{a} \cdot \bar{n}}{r} d S, \\
\vec{\alpha}(\vec{x}) & =\frac{1}{4 \pi} \iiint_{V} \frac{\nabla \times \vec{a}}{r} d V+\frac{1}{4 \pi} \iint_{\partial V} \frac{\vec{a} \times \bar{n}}{r} d S .
\end{aligned}
$$
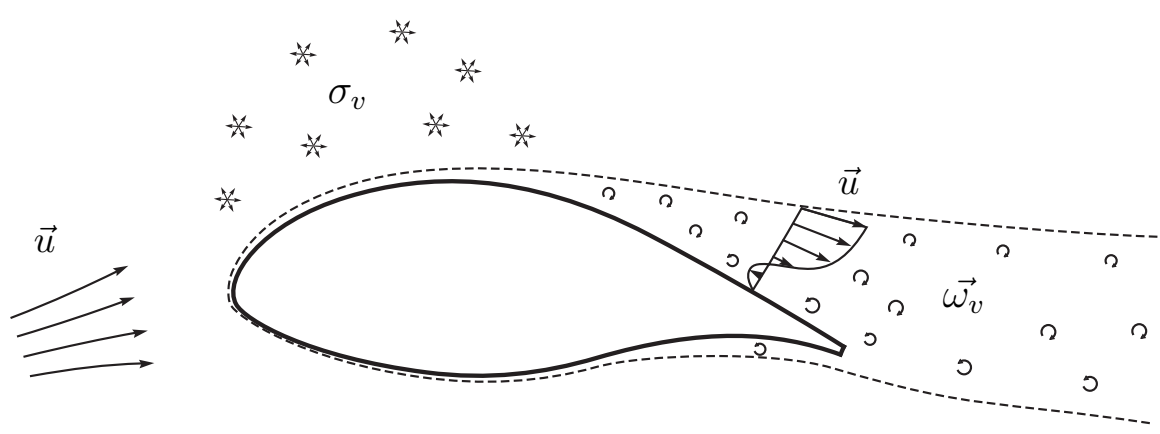

Figure C.1: Helmholtz' decomposition for the velocity field.

For boundary $\partial V$ tending to infinity and the condition that the vector field $\vec{a}(\vec{y})$ is decaying faster to zero than $1 / r$ we have for $\vec{x} \in V$

$$
\begin{aligned}
\psi(\vec{x}) & =\frac{-1}{4 \pi} \iiint_{V} \frac{\nabla \cdot \vec{a}}{r} d V, \\
\vec{\alpha}(\vec{x}) & =\frac{1}{4 \pi} \iiint_{V} \frac{\nabla \times \vec{a}}{r} d V,
\end{aligned}
$$


where

$$
\vec{r}=\vec{x}-\vec{y}, \quad r=|\vec{r}| .
$$

Using Helmholtz' theorem, a continuously differentiable velocity vector field $\vec{u}(\vec{x})$ can be composed from a velocity field $\nabla \Phi_{\infty}(\vec{x})$ that is both irrotational and solenoidal, and two perturbation fields involving the divergence and the curl of the velocity vector field in the volume as depicted in Figure C.1, that is,

$$
\vec{u}(\vec{x})=\nabla \Phi_{\infty}(\vec{x})+\iiint_{V} \frac{\sigma_{v} \vec{r}}{4 \pi r^{3}} d V+\iiint_{V} \frac{\overrightarrow{\omega_{v}} \times \vec{r}}{4 \pi r^{3}} d V,
$$

where the volumetric source and vorticity distributions are respectively

$$
\begin{aligned}
& \sigma_{v}(\vec{y})=\nabla \cdot \vec{u}, \\
& \vec{\omega}_{v}(\vec{y})=\nabla \times \vec{u} .
\end{aligned}
$$

Restricting source and vorticity volume distributions to the body and wake surfaces $\partial V$ will lead to divergence free and irrotational flow everywhere except across these surfaces as indicated in Figure C.2. In formal notation this means that we define the surface source and vorticity distributions $\sigma\left(\vec{y}_{S}\right)$ and $\vec{\gamma}\left(\vec{y}_{S}\right)$ as functions of volume distributions $\sigma_{v}(\vec{y})$ and $\vec{\omega}_{v}(\vec{y})$ by

$$
\begin{aligned}
& \sigma_{v}(\vec{y})=\sigma\left(\vec{y}_{S}\right) \delta\left(\vec{y}_{S}-\vec{y}\right), \\
& \vec{\omega}_{v}(\vec{y})=\vec{\gamma}\left(\vec{y}_{S}\right) \delta\left(\vec{y}_{S}-\vec{y}\right),
\end{aligned}
$$

where $\vec{y}_{S} \in \partial V$ and where $\delta\left(\vec{y}_{S}-\vec{y}\right)$ is the three-dimensional Dirac delta function, the product of the delta functions for the three Cartesian components. The resulting expression for the potential velocity $\vec{u}(\vec{x})=\nabla \Phi(\vec{x})$ is now

$$
\nabla \Phi(\vec{x})=\nabla \Phi_{\infty}(\vec{x})+\iint_{\partial V} \frac{\sigma \vec{r}}{4 \pi r^{3}} d S+\iint_{\partial V} \frac{\vec{\gamma} \times \vec{r}}{4 \pi r^{3}} d S,
$$

where

$$
\vec{r}=\vec{x}-\vec{y}_{S}, \quad r=|\vec{r}| .
$$

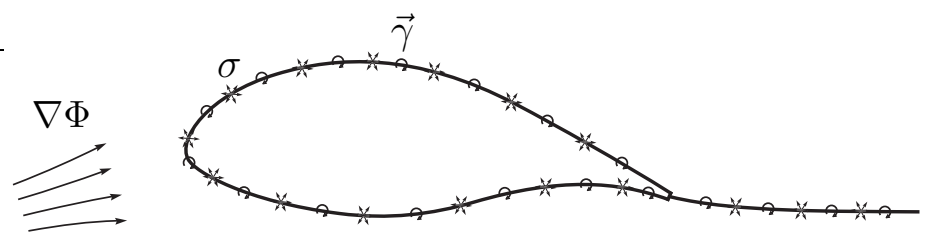

Figure C.2: Potential flow velocity field. 


\section{Rotational Onset Flow}

Wind turbines are normally operating in unsteady wind with shear in which the wind field has a rotational component. This section follows the analysis by Keenan [64] and investigates the conditions under which it is justifiable to apply a potential flow panel method to the simulation of the flow around wind turbine rotors when submerged in a rotational onset flow.

In incompressible flows subjected to a conservative force field the evolution of the vorticity field $\vec{\omega}(\vec{x}, t)$ can be obtained by applying the curl operator to the equations expressing the conservation of momentum (B.13). After some manipulation, the resulting Eulerian description for inviscid flows is

$$
\frac{\partial \vec{\omega}}{\partial t}+\vec{u} \cdot \nabla \vec{\omega}=\vec{\omega} \cdot \nabla \vec{u}
$$

We split the flow field into a rotational onset flow field $\vec{u}_{0}$ and a perturbation velocity field $\vec{u}_{1}$ due to the presence of the wind turbine that will be captured by the panel method:

$$
\vec{u}=\vec{u}_{0}+\vec{u}_{1} .
$$

The corresponding vorticity distribution is $\vec{\omega}=\vec{\omega}_{0}+\vec{\omega}_{1}$, in which

$$
\vec{\omega}_{0}=\nabla \times \vec{u}_{0}, \quad \text { and } \quad \vec{\omega}_{1}=\nabla \times \vec{u}_{1} .
$$

The term $\vec{\omega}_{1}$ is due to the wake surfaces emanation from the lifting components of the configuration.

Substituting (D.2) and (D.3) in (D.1) gives

$$
\frac{\partial\left(\vec{\omega}_{0}+\vec{\omega}_{1}\right)}{\partial t}+\left(\vec{u}_{0}+\vec{u}_{1}\right) \cdot \nabla\left(\vec{\omega}_{0}+\vec{\omega}_{1}\right)=\left(\vec{\omega}_{0}+\vec{\omega}_{1}\right) \cdot \nabla\left(\vec{u}_{0}+\vec{u}_{1}\right) .
$$

For the onset wind field $\vec{u}_{0}$ with rotational components it is assumed that the equation for conservation of momentum for inviscid flow (D.1) is satisfied, so that

$$
\frac{\partial \vec{\omega}_{0}}{\partial t}+\vec{u}_{0} \cdot \nabla \vec{\omega}_{0}=\vec{\omega}_{0} \cdot \nabla \vec{u}_{0}
$$

Expanding the terms in (D.4) and substituting (D.5) gives an equation for the perturbation flow field:

$$
\frac{\partial \vec{\omega}_{1}}{\partial t}+\vec{u}_{1} \cdot \nabla \vec{\omega}_{1}-\vec{\omega}_{1} \cdot \nabla \vec{u}_{1}=-\vec{u}_{1} \cdot \nabla \vec{\omega}_{0}-\vec{u}_{0} \cdot \nabla \vec{\omega}_{1}+\vec{\omega}_{1} \cdot \nabla \vec{u}_{0}+\vec{\omega}_{0} \cdot \nabla \vec{u}_{1} .
$$


For a thick rotor blade in a rotational onset flow the blade chord length $c[\mathrm{~m}]$ is set as the characteristic length scale for the perturbation flow field. Let the characteristic perturbation velocity be $\lambda U_{0}\left[\mathrm{~m} \cdot \mathrm{s}^{-1}\right]$, with $\lambda$ a scaling factor equal to ratio of the local surface velocity and the onset wind speed. For current wind turbine designs the local speed ratio increases from $\lambda \approx 1$ at the blade root to $\lambda \approx 8$ at the tip.

Let the characteristic velocity of the onset flow be $U_{0}\left[\mathrm{~m} \cdot \mathrm{s}^{-1}\right]$, and let $\Delta[\mathrm{m}]$ be the characteristic length scale for the onset flow wind shear, where $\Delta \gg c$. With these scales the order of magnitude of the cross terms in the right-hand side of equation (D.6) can be estimated:

$$
\begin{aligned}
& \frac{\left|\vec{u}_{1} \cdot \nabla \vec{\omega}_{0}\right|}{\left|\vec{u}_{1} \cdot \nabla \vec{\omega}_{1}\right|} \approx \mathcal{O}\left(\frac{1}{\lambda} \frac{c^{2}}{\Delta^{2}}\right) \\
& \frac{\left|\vec{u}_{0} \cdot \nabla \vec{\omega}_{1}\right|}{\left|\vec{u}_{1} \cdot \nabla \vec{\omega}_{1}\right|} \approx \mathcal{O}\left(\frac{1}{\lambda}\right) \\
& \frac{\left|\vec{\omega}_{1} \cdot \nabla \vec{u}_{0}\right|}{\left|\vec{u}_{1} \cdot \nabla \vec{\omega}_{1}\right|} \approx \mathcal{O}\left(\frac{1}{\lambda} \frac{c}{\Delta}\right) \\
& \frac{\left|\vec{\omega}_{0} \cdot \nabla \vec{u}_{1}\right|}{\left|\vec{u}_{1} \cdot \nabla \vec{\omega}_{1}\right|} \approx \mathcal{O}\left(\frac{1}{\lambda} \frac{c}{\Delta}\right)
\end{aligned}
$$

Ignoring the terms smaller than $\mathcal{O}(1 / \lambda)$, but retaining the term $\vec{\omega}_{1} \cdot \nabla \vec{u}_{0}$, reduces equation (D.6) to

$$
\frac{\partial \vec{\omega}_{1}}{\partial t}+\left(\vec{u}_{0}+\vec{u}_{1}\right) \cdot \nabla \vec{\omega}_{1}=\vec{\omega}_{1} \cdot \nabla\left(\vec{u}_{0}+\vec{u}_{1}\right) .
$$

Equation (D.7) states that the convection and stretching of the wake vorticity is due to the total velocity field.

The terms in equation (D.6) that were neglected are $\vec{u}_{1} \cdot \nabla \vec{\omega}_{0}$ and $\vec{\omega}_{0} \cdot \nabla \vec{u}_{1}$, which are respectively the contributions to the convection and the stretching of the onset flow field vorticity due to the perturbation velocity field. We thus ignore the influence of wind turbine on the onset flow vorticity development. This approximation is justified as long as the characteristic length scale in the onset wind is much larger than the rotor blade chord length .

A similar analysis can be performed for the dynamics of the wake in a rotational onset flow field. We follow the analysis by Keenan [64] and let circulation $\Gamma\left[\mathrm{m}^{2} \cdot \mathrm{s}^{-1}\right]$ be characteristic for the lifting flow of the configuration and set $\delta[\mathrm{m}]$ the characteristic thickness of the wake vortex sheet. Combined these scales give a characteristic velocity $\Gamma / \delta$ for the perturbation flow field. The estimate for the order of magnitude of the cross terms 
in the right-hand side of equation (D.6) is now

$$
\begin{aligned}
& \frac{\left|\vec{u}_{1} \cdot \nabla \vec{\omega}_{0}\right|}{\left|\vec{u}_{1} \cdot \nabla \vec{\omega}_{1}\right|} \approx \mathcal{O}\left(U_{0} \frac{\delta}{\Gamma} \frac{\delta^{2}}{\Delta^{2}}\right) \\
& \frac{\left|\vec{u}_{0} \cdot \nabla \vec{\omega}_{1}\right|}{\left|\vec{u}_{1} \cdot \nabla \vec{\omega}_{1}\right|} \approx \mathcal{O}\left(U_{0} \frac{\delta}{\Gamma}\right) \\
& \frac{\left|\vec{\omega}_{1} \cdot \nabla \vec{u}_{0}\right|}{\left|\vec{u}_{1} \cdot \nabla \vec{\omega}_{1}\right|} \approx \mathcal{O}\left(U_{0} \frac{\delta}{\Gamma} \frac{\delta}{\Delta}\right) \\
& \frac{\left|\vec{\omega}_{0} \cdot \nabla \vec{u}_{1}\right|}{\left|\vec{u}_{1} \cdot \nabla \vec{\omega}_{1}\right|} \approx \mathcal{O}\left(U_{0} \frac{\delta}{\Gamma} \frac{\delta}{\Delta}\right)
\end{aligned}
$$

The analysis leads again to equation (D.7) if we ignore the terms smaller than $\mathcal{O}\left(U_{0} \delta / \Gamma\right)$, but retain the term $\vec{\omega}_{1} \cdot \nabla \vec{u}_{0}$. Discarding the terms $\vec{u}_{1} \cdot \nabla \vec{\omega}_{0}$ and $\vec{\omega}_{0} \cdot \nabla \vec{u}_{1}$ is justified as long as $\Delta \gg \delta$.

For turbulent length scales in the onset wind velocity of the order of the boundary layer thickness at the rotor blade the decomposition of the flow field into two components is not justified anymore and the full velocity field should be accounted for. 


\section{E Boundary Integral Equation}

In an inertial Cartesian coordinate system in 3D space in which coordinates are denoted by $\vec{x}=(x, y, z)^{\mathrm{T}}$, the Laplace equation for the velocity potential in unsteady, incompressible flow, in domain $V \in \mathbb{R}^{3}$, can be written as

$$
\nabla \cdot \nabla \Phi=\frac{\partial^{2} \Phi}{\partial x^{2}}+\frac{\partial^{2} \Phi}{\partial y^{2}}+\frac{\partial^{2} \Phi}{\partial z^{2}}=0
$$

Although the Laplace equation has no time-dependent term, the velocity potential $\Phi(\vec{x}, t)$ is a function of space and time. Unsteady boundary conditions will introduce time-dependence in the solution. In this section, timedependence is implicitly assumed. The problem in volume $V$ can be reduced to a problem involving only surface integrals by the introduction of a form of the Divergence Theorem that relates the volume and surface integrals of an arbitrary tensor function over a volume $V$ enclosed by surface $\partial V$ with unit normal vector $\bar{n}$ pointing into the volume:

$$
\iint_{\partial V}\left(\Psi_{2} \nabla \Psi_{1}-\Psi_{1} \nabla \Psi_{2}\right) \cdot \bar{n} d S=\iiint_{V}\left(\Psi_{1} \nabla^{2} \Psi_{2}-\Psi_{2} \nabla^{2} \Psi_{1}\right) d V
$$

equation (E.2) is known as Green's second identity in which surface $\partial V$ is piecewise continuous and the scalar functions $\Psi_{1}$ and $\Psi_{2}$ are assumed to be twice continuously differentiable.

We set for $3 \mathrm{D}$ flows

$$
\Psi_{1}=\frac{1}{r}, \quad \Psi_{2}=\Phi,
$$

in which $\Phi(\vec{x})$ is the velocity potential function at point $\vec{x}$ (see Figure E.1) and $r$ is the distance from point $\vec{y}$ on the enclosing surface to an arbitrary fixed point $\vec{x}$ :

$$
\vec{r}=\vec{x}-\vec{y}, \quad r=|\vec{r}|, \quad \text { where } \quad \vec{y} \in \partial V .
$$

Notice that, taking derivatives with respect to $\vec{x}$, that

$$
\nabla \frac{1}{r}=-\frac{\vec{r}}{r^{3}}, \quad \text { and } \quad \nabla^{2} \frac{1}{r}=0, \quad \text { for } \quad r \neq 0 .
$$

For point $\vec{x}$ outside flow domain $V$ both $\Psi_{1}$ and $\Psi_{2}$ satisfy the Laplace equation and equation (E.2) becomes

$$
\iint_{\partial V}\left(\Phi \nabla \frac{1}{r}-\frac{1}{r} \nabla \Phi\right) \cdot \bar{n} d S=0, \quad \vec{x} \notin V .
$$




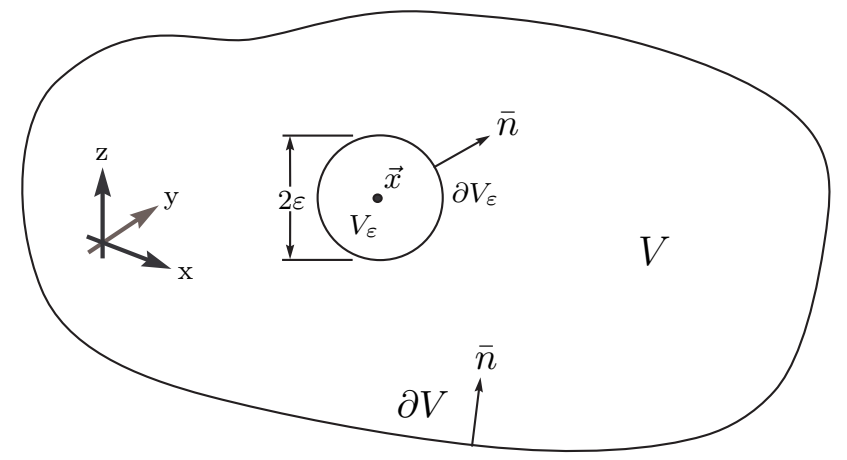

Figure E.1: Flow domain with $\vec{x} \in V$ excluded from the domain of integration.

In case point $\vec{x}$ is inside the domain of interest $V$, a small sphere with radius $\varepsilon$ is introduced to exclude volume $V_{\varepsilon}$ around point $\vec{x}$ from the volume of integration (see Figure E.1). Again, both $\Psi_{1}$ and $\Psi_{2}$ satisfy the Laplace equation and equation (E.2) now becomes

$$
\iint_{\partial V+\partial V_{\varepsilon}}\left(\Phi \nabla \frac{1}{r}-\frac{1}{r} \nabla \Phi\right) \cdot \bar{n} d S=0, \quad \vec{x} \in V .
$$

Evaluation of the integral over the surface of the sphere $\partial V_{\varepsilon}$, using equation (E.5), gives us

$$
\iint_{\partial V_{\varepsilon}}\left(\Phi \nabla \frac{1}{r}-\frac{1}{r} \nabla \Phi\right) \cdot \bar{n} d S=\iint_{\partial V_{\varepsilon}}\left(\Phi \frac{\bar{n} \cdot \vec{r}}{r^{3}}-\frac{1}{r} \nabla \Phi \cdot \bar{n}\right) d S .
$$

For a sphere with radius $\varepsilon$ we have $\int d S=4 \pi \varepsilon^{2}$. Assuming a continuously differentiable velocity potential $\Phi$ and letting $\varepsilon \rightarrow 0$, the second term in equation (E.8) vanishes:

$$
\iint_{\partial V_{\varepsilon}}\left(-\frac{1}{r} \nabla \Phi \cdot \bar{n}\right) d S=-\frac{\partial \Phi}{\partial n} \iint_{\partial V_{\varepsilon}}\left(\frac{1}{r}\right) d S=0,
$$

and for the first term in (E.8) we find

$$
\iint_{\partial V_{\varepsilon}}\left(\Phi \frac{\bar{n} \cdot \vec{r}}{r^{3}}\right) d S=-\Phi(\vec{x}) \iint_{\partial V_{\varepsilon}}\left(\frac{1}{r^{2}}\right) d S=-4 \pi \Phi(\vec{x}),
$$

where we used for the normal vector on the sphere the expression

$$
\bar{n}=\frac{\vec{y}-\vec{x}}{|\vec{y}-\vec{x}|}=-\frac{\vec{r}}{r}
$$


Substitution of (E.9) and (E.10) in equation (E.7) gives an expression for the velocity potential $\Phi$ at an arbitrary point $\vec{x} \in V$ in terms of an integral over the boundaries $\partial V$ :

$$
\Phi(\vec{x})=\frac{1}{4 \pi} \iint_{\partial V}\left(\Phi \nabla \frac{1}{r}-\frac{1}{r} \nabla \Phi\right) \cdot \bar{n} d S, \quad \vec{x} \in V .
$$

The same procedure can be followed for point $\vec{x}$ located at $\partial V$. Now only half a sphere is surrounding $\vec{x}$, assuming that $\vec{x}$ is not located at a surfaceslope discontinuity. In addition, the intersection of the hemisphere with $\partial V$ is excluded from the surface of integration (see Figure E.2). This results in the following integral:

$$
\Phi(\vec{x})=\frac{1}{2 \pi} \iint_{\partial V}\left(\Phi \nabla \frac{1}{r}-\frac{1}{r} \nabla \Phi\right) \cdot \bar{n} d S, \quad \vec{x} \in \partial V .
$$

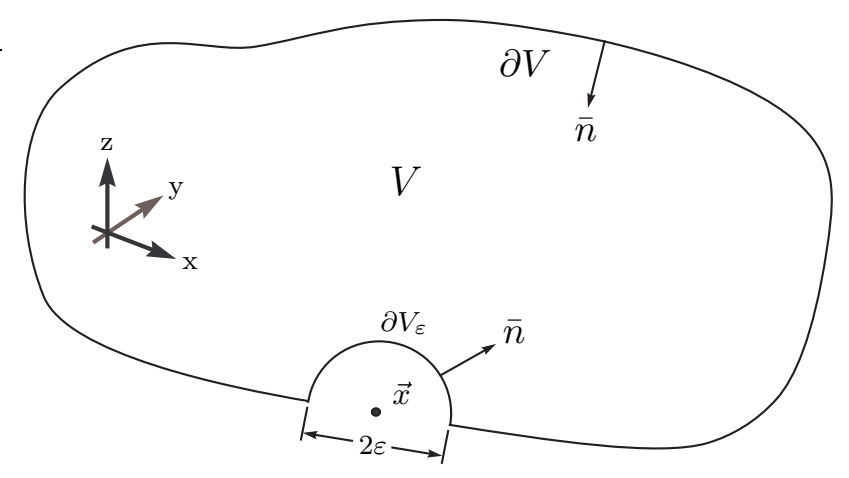

Figure E.2: Flow domain with $\vec{x} \in \partial V$ excluded from the domain of integration.

In summary, we have found that

$$
\frac{1}{4 \pi} \iint_{\partial V}\left(\Phi \nabla \frac{1}{r}-\frac{1}{r} \nabla \Phi\right) \cdot \bar{n} d S= \begin{cases}0 & \vec{x} \notin V, \\ \frac{1}{2} \Phi(\vec{x}) & \vec{x} \in \partial V, \\ \Phi(\vec{x}) & \vec{x} \in V .\end{cases}
$$

Now, consider the example situation in Figure E.3 in which we have a collection of non-overlapping subdomains $V_{m}$, each with a velocity potential function $\Phi_{m}$. Some of the flow domains will have physical significance, while others are introduced to allow for certain boundary conditions, or for their influence on external regions. The boundary separating volume $V_{m}$ from volume $V_{k}$ is denoted by $S_{m, k}$

$$
S_{m, k}=\partial V_{m} \cap \partial V_{k}, \quad m \neq k,
$$


and normal vector $\bar{n}_{m}$ is defined to point into subdomain $V_{m}$. The surface $S$ of the configuration and its wake (see Figure E.3) are described by the complete set of inner boundaries:

$$
S=\cup S_{m, k}
$$

The outer boundary $S_{0,1}$ is located either a finite or an infinite distance away from the inner boundaries in case of internal or external flows respectively.

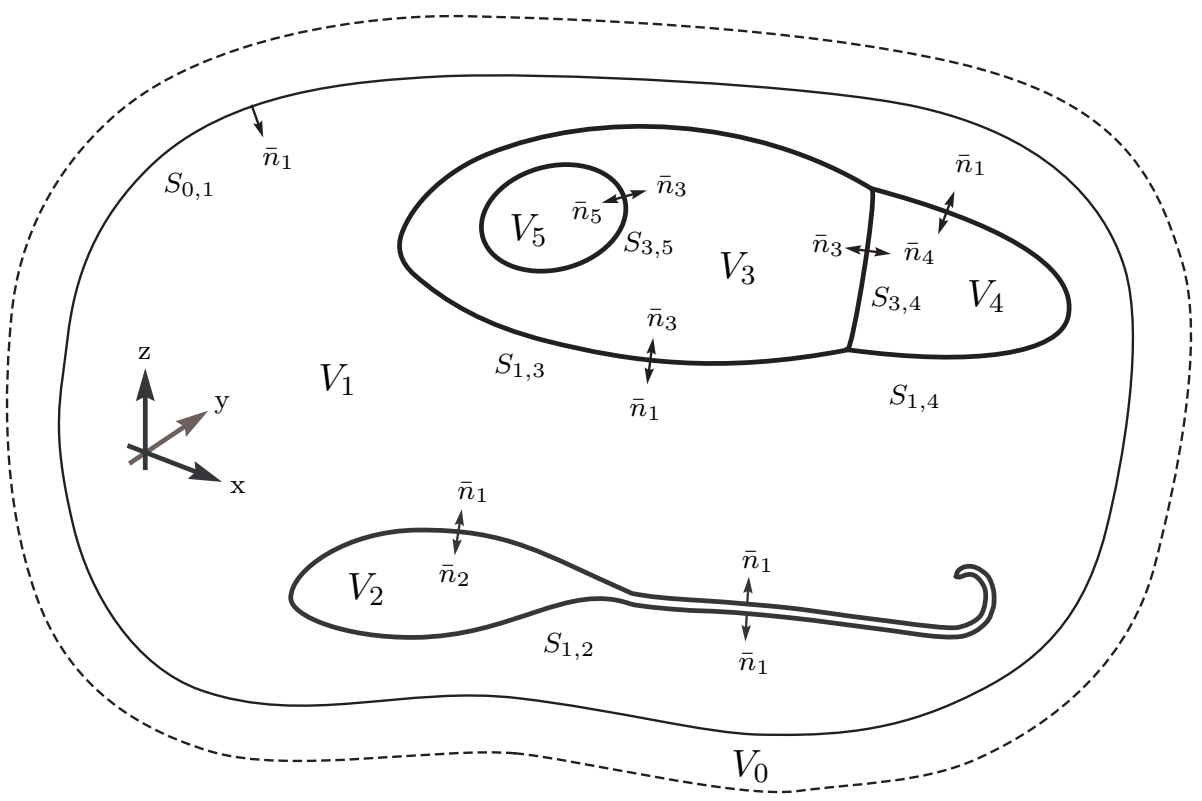

Figure E.3: Flow domain composed of non-overlapping volumes $V_{m}$ and boundaries $S_{m, k}$ that separate volume $V_{m}$ from volume $V_{k}$.

In the situation where point $\vec{x}$ lies in subdomain $V_{1}$, the contributions to equation (E.14) from the boundaries of volumes $V_{1}, V_{2}, V_{3}, V_{4}$ and $V_{5}$ are respectively

$$
\begin{aligned}
& \Phi(\vec{x})=\frac{1}{4 \pi} \iint_{S_{0,1}+S_{1,2}+S_{1,3}+S_{1,4}}\left(\Phi_{1} \nabla \frac{1}{r}-\frac{1}{r} \nabla \Phi_{1}\right) \cdot \bar{n}_{1} d S, \\
& 0=\frac{1}{4 \pi} \iint_{S_{1,2}}\left(\Phi_{2} \nabla \frac{1}{r}-\frac{1}{r} \nabla \Phi_{2}\right) \cdot \bar{n}_{2} d S, \\
& 0=\frac{1}{4 \pi} \iint_{S_{1,3}+S_{3,4}+S_{3,5}}\left(\Phi_{3} \nabla \frac{1}{r}-\frac{1}{r} \nabla \Phi_{3}\right) \cdot \bar{n}_{3} d S, \quad \text { for } \quad \vec{x} \in V_{1} \text {. } \\
& 0=\frac{1}{4 \pi} \iint_{S_{1,4}+S_{3,4}}\left(\Phi_{4} \nabla \frac{1}{r}-\frac{1}{r} \nabla \Phi_{4}\right) \cdot \bar{n}_{4} d S, \\
& 0=\frac{1}{4 \pi} \iint_{S_{3,5}}\left(\Phi_{5} \nabla \frac{1}{r}-\frac{1}{r} \nabla \Phi_{5}\right) \cdot \bar{n}_{5} d S,
\end{aligned}
$$


For the situation where point $\vec{x}$ lies in subdomain $V_{k}$, we end up with almost the same set of equations; only the factor $\Phi(\vec{x})$ on the left hand side shifts to the equation of region $V_{k}$. Summation of the integral equations in equation (E.17) gives us therefore the following general formula for the velocity potential at a fixed point $\vec{x} \in V$, where we use the relationship $\bar{n}_{k}=-\bar{n}_{m}$ for $\vec{x} \in S_{m, k}$ :

$$
\begin{aligned}
\Phi(\vec{x})= & \frac{1}{4 \pi} \iint_{S}\left(\left(\Phi_{m}-\Phi_{k}\right) \nabla \frac{1}{r}-\frac{1}{r} \nabla\left(\Phi_{m}-\Phi_{k}\right)\right) \cdot \bar{n}_{m} d S+ \\
& \frac{1}{4 \pi} \iint_{S_{0,1}}\left(\Phi_{1} \nabla \frac{1}{r}-\frac{1}{r} \nabla \Phi_{1}\right) \cdot \bar{n}_{1} d S, \quad \vec{x} \in V
\end{aligned}
$$

In the limit where the distance of the outer boundary $S_{0,1}$ to the inner boundaries goes to infinity, the contribution to $\Phi(\vec{x})$ from the integral in equation (E.18) for $S_{0,1}$ can be considered to represent the unperturbed velocity potential $\Phi_{\infty}(\vec{x})$ in the entire domain, that is, the velocity potential if no inner boundaries were present. The integral contributions from the inner boundaries may thus be considered perturbation velocity potentials. Of course this is only valid if the velocity potential perturbation in equation (E.18) vanishes at infinity.

In the case that boundary $S_{0,1}$ lies within a finite distance from the other boundaries, as is the case for internal flows, a region $V_{0}$ (the dotted line in figure E.3) may be considered to lie outside $S_{0,1}$ that extends to infinity and gives rise to $\Phi_{\infty}(\vec{x})$ again. The surface $S_{0,1}$ is now part of the inner boundaries (E.16).

If we define the dipole and source surface singularity strengths to be

$$
\mu=-\left(\Phi_{m}-\Phi_{k},\right) \quad \text { and } \quad \sigma=\nabla\left(\Phi_{m}-\Phi_{k}\right) \cdot \bar{n}_{m},
$$

and, using the definition for $\vec{r}$ in equation (E.4), introduce the dipole and source perturbation velocity potentials

$$
\begin{aligned}
\varphi_{\mu}(\vec{x}) & =\frac{-1}{4 \pi} \iint_{S} \mu \frac{\bar{n}_{m} \cdot \vec{r}}{r^{3}} d S, \\
\varphi_{\sigma}(\vec{x}) & =\frac{-1}{4 \pi} \iint_{S} \sigma \frac{1}{r} d S,
\end{aligned}
$$

we can reformulate boundary integral equation (E.18) as

$$
\Phi(\vec{x})=\Phi_{\infty}(\vec{x})+\varphi_{\mu}(\vec{x})+\varphi_{\sigma}(\vec{x}), \quad \vec{x} \in V .
$$


It is also possible to include the influence of source and dipole singularities in the near field in the definition of the onset velocity potential field as long as (E.22) is not evaluated there.

The perturbation velocity potential functions in equations (E.20) and (E.21) vanish towards infinity and satisfy the far-field condition mentioned earlier. The integrals have a singular integrand which results in a jump in velocity potential of magnitude $\mu$ at point $\vec{y} \in S_{m, k}$ across the surface:

$$
\lim _{\varepsilon \rightarrow 0} \varphi\left(\vec{y} \pm \varepsilon \bar{n}_{m}, t\right)=\varphi_{\sigma}^{p}(\vec{y}, t)+\varphi_{\mu}^{p}(\vec{y}, t) \mp \frac{1}{2} \mu(\vec{y}, t) .
$$

The two $\varphi^{p}\left(\vec{y} \in S_{m, k}\right)$ terms are to be interpreted as Principal Value or Finite Part integrals in which a small region around the singular point is excluded from the surface of integration. 


\section{F Barycentric Lagrange Interpolation}

A short description is given of barycentric Lagrange interpolation as advocated by Berrut and Trefethen [9]. For multivariate functions a tensor product approach is described that makes use of repeated invocations of univariate Lagrange interpolation.

Given a set of $n$ nodes $x_{i}, i=1, \ldots, n$ together with corresponding function values $f_{i}$. The problem is to find a univariate polynomial $p(x)$ that interpolates through the function values, that is $p\left(x_{i}\right)=f_{i}$. The interpolation polynomial $p_{n}(x)$ through the set of $n$ points $x_{i}$ can be written as

$$
p_{n}(x)=\sum_{i=1}^{n} L_{i}^{n}(x) f_{i},
$$

where the Lagrange polynomial $L_{i}^{n}(x)$ has the property

$$
L_{i}^{n}\left(x_{k}\right)=\delta_{i k} .
$$

The Lagrange polynomial basis functions can be written in barycentric form $[9]$ as

$$
L_{i}^{n}(x)=\frac{\frac{w_{i}}{x-x_{i}}}{\sum_{j=1}^{n} \frac{w_{j}}{x-x_{j}}},
$$

where the weights $w_{i}$ are defined up to an arbitrary multiplication factor by

$$
w_{i}=\frac{1}{\prod_{\substack{j=1 \\ j \neq i}}^{m} x_{i}-x_{j}}, \quad i=1, \ldots, n .
$$

The work to compute all weights $w_{i}$ is $\mathcal{O}\left(n^{2}\right)$ and independent of $x$. Once the weights are known the remaining work to interpolate in $x$ is of $\mathcal{O}(n)$. For Chebyshev node distributions of the first kind the weights are, up to an arbitrary multiplication factor, given by the explicit formula

$$
w_{i}=(-1)^{i} \sin \frac{(2 i-1) \pi}{2 n}, \quad i=1, \ldots, n,
$$

with the nodes distributed in domain $[-1,1]$ according to

$$
x_{i}=\cos \frac{(2 n-2 i+1) \pi}{2 n}, \quad i=1, \ldots, n .
$$


An example of the basis Lagrange polynomials $L_{i}^{n}(x)$ through five Chebyshev nodes of the first kind is given in Figure F.1.

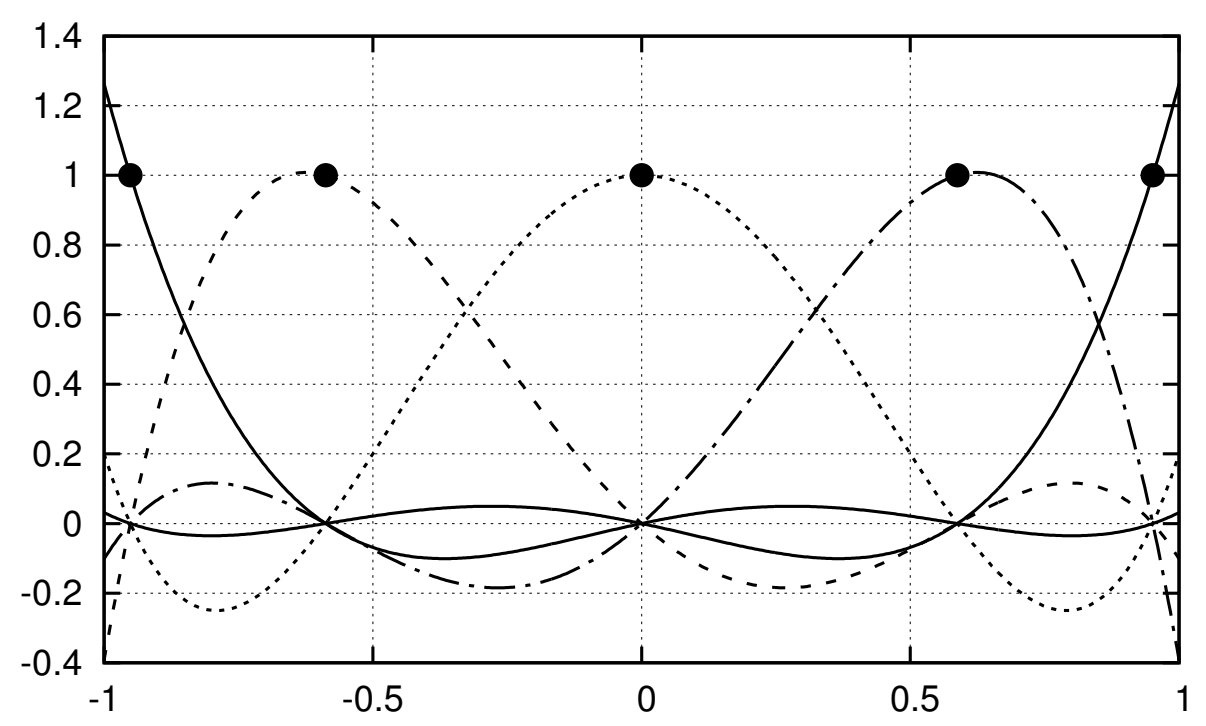

Figure F.1: Basis Lagrange polynomials $L_{i}^{5}(x)$ through five Chebyshev nodes of the first kind.

The error made at a point $x$ in interval $[a, b]$ by the approximation of function $f(x)$ with a polynomial $p_{n}(x)$ that interpolates through $n$ function values $f\left(x_{i}\right)$ with $x_{i} \in[a, b]$ is

$$
\epsilon_{n}(x)=f(x)-p_{n}(x)=\frac{f^{(n)}(\xi)}{n !} \prod_{i=1}^{n}\left(x-x_{i}\right),
$$

for some $\xi \in[a, b]$. For a Chebyshev node distribution of the first kind a bound can be derived for the product term in equation (F.7) which results in an error bounded for $x \in[a, b]$ by

$$
\left|\epsilon_{n}(x)\right| \leq \frac{1}{2^{2 n-1} n !}(b-a)^{n} \max \left|f^{(n)}(\xi)\right| .
$$

The Lebesgue constant, a measure for the stability of a polynomial representation of a continuous function through a predefined set of nodes on interval $[a, b]$, is defined by

$$
\Lambda_{n}=\max _{x \in[a, b]} \sum_{i=1}^{n}\left|L_{i}^{n}(x)\right|,
$$


and appears in the inequality

$$
\max _{x \in[a, b]}\left|p_{n}(x)\right| \leq \Lambda_{n} \max _{x \in[a, b]}|f(x)| .
$$

For Chebyshev nodes of the first kind an upper bound for the Lebesgue constant for $n \geq 2$ is given by

$$
\Lambda_{n} \leq \frac{2}{\pi} \ln n+1
$$

For the first derivative of the polynomial interpolant (F.1) we have

$$
p_{n}^{\prime}(x)=\sum_{i=1}^{n} L_{i}^{\prime}(x) f_{i},
$$

and after some manipulations we find for the first derivatives of the basis polynomials $L_{i}^{\prime}\left(x_{j}\right)$ in the interpolation nodes $x_{j}$ themselves

$$
L_{i}^{\prime}\left(x_{j}\right)=\frac{w_{i} / w_{j}}{x_{i}-x_{j}}, \quad \text { for } i \neq j,
$$

and for the case $i=j$ we find

$$
L_{i}^{\prime}\left(x_{i}\right)=-\sum_{j \neq i}^{n} L_{i}^{\prime}\left(x_{j}\right)
$$

For interpolation in multiple variables we can make use of tensor products and use one-dimensional interpolation in each variable direction. For Lagrange interpolation in two variables $x$ and $y$ this results with $p\left(x_{i}, y_{j}\right)=f_{i j}$ in

$$
p(x, y)=\sum_{i=1}^{n} \sum_{j=1}^{m} L_{i}^{n}(x) L_{j}^{m}(y) f_{i j},
$$

where the Lagrange polynomials $L_{i}^{n}(x)$ and $L_{j}^{m}(y)$ have the property

$$
L_{i}^{n}\left(x_{k}\right)=\delta_{i k}, \quad \text { and } \quad L_{j}^{m}\left(y_{k}\right)=\delta_{j k}
$$

For computational purposes it is useful to reorder equation (F.15) to

$$
p(x, y)=\sum_{i=1}^{n} L_{i}^{n}(x) \sum_{j=1}^{m} L_{j}^{m}(y) f_{i j},
$$

or equivalently to

$$
p(x, y)=\sum_{j=1}^{m} L_{j}^{m}(y) \sum_{i=1}^{n} L_{i}^{n}(x) f_{i j} .
$$


The algorithm to interpolate in a $2 \mathrm{D}$ array of data points is illustrated in Figure F.2. Following equation (F.17) first the function values are interpolated in $y$ along lines of varying $j$ giving the data points represented by the circles. These data points are then interpolated in $x$ giving the function value at point $(x, y)$.

In the procedure following equation (F.18) first the data is interpolated along lines of varying index $i$ giving the data points represented by the square symbols. The so obtained data values are then interpolated in $y$ to give the function value at point $(x, y)$.

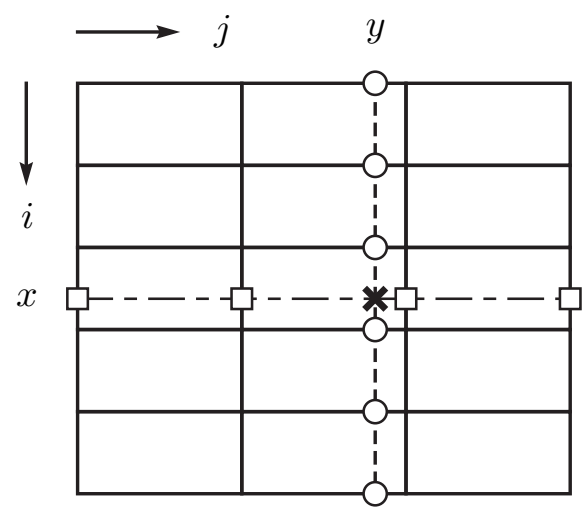

Figure F.2: Tensor product interpolation in an array of data points.

Note that when $x=x_{k}$, that is when $x$ aligns with the original data points, the summation over $i$ in equations (F.17) and (F.18) disappears due to property (F.16) and we can set $i=k$. Similarly, when $y=y_{k}$ the summation over $j$ in equations (F.17) and (F.18) disappears and $j=k$ can be used. 


\section{G Tri-Axial Ellipsoids}

In this appendix we will discuss the analytical potential flow solution, known from literature, for a tri-axial ellipsoid (see Figure G.1) in arbitrary uniform onset flow, and in still air rotating around an arbitrary axis through its center. For sake of completeness equivalent expressions are derived for the $2 \mathrm{D}$ problem by letting one of the ellipsoid dimensions approach infinity.

The analytical solutions make it possible to verify the implementation of numerical potential flow methods for the correctness of system of equation setup, the solution of the system by a linear equation solver, and the application of post-processing steps necessary to obtain the surface velocity distribution. A quantitative estimate of the errors introduced by the numerical scheme will thus be obtained. The combination of the two exact solutions can describe any flow case with uniform onset flow with rotation of the ellipsoid around an axis of arbitrary direction and location.

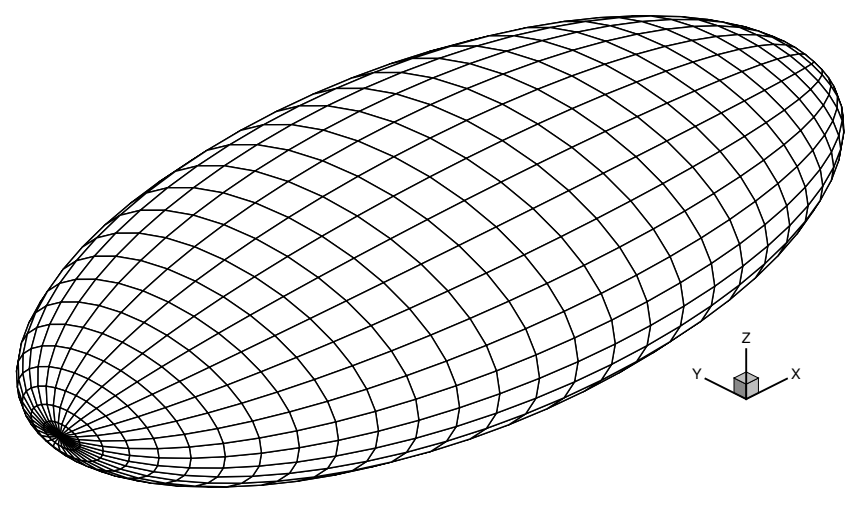

Figure G.1: Tri-axial ellipsoid with semi-axes $(4,2,1)$ and a surface discretization of $32 \times 32$ panels. 


\section{G.1 Ellipsoid Geometry}

The surface of an ellipsoid with semi-axes $(a, b, c)$ is described by $\psi\left(\vec{x}_{e}\right)=0$ if we define the function $\psi(\vec{x})$ as

$$
\psi(\vec{x})=\left(\frac{x}{a}\right)^{2}+\left(\frac{y}{b}\right)^{2}+\left(\frac{z}{c}\right)^{2}-1
$$

where $\vec{x}_{e}=\left(x_{e}, y_{e}, z_{e}\right)^{\mathrm{T}}$ is a point at the ellipsoid surface. The unit normal vector to the surface of the ellipsoid $\bar{n}\left(\vec{x}_{e}\right)$ can be calculated from

$$
\bar{n}\left(\vec{x}_{e}\right)=\frac{\nabla \psi\left(\vec{x}_{e}\right)}{\left|\nabla \psi\left(\vec{x}_{e}\right)\right|} .
$$

Using the implicit definition of the ellipsoid surface (G.1), the gradient of function $\psi$ evaluated at the surface of the ellipsoid is given by

$$
\nabla \psi\left(\vec{x}_{e}\right)=\left(\frac{2 x_{e}}{a^{2}}, \frac{2 y_{e}}{b^{2}}, \frac{2 z_{e}}{c^{2}}\right)^{\mathrm{T}}
$$

In some cases the points where we know a numerical solution are not located exactly on the ellipsoid surface but lie at some small distance from it. Projection of these points onto the surface of the ellipsoid gives us again a location where we can determine the exact solution of the flow problem. For example, points $\vec{x}_{e}$ on the ellipsoid surface are obtained by projecting the collocation points $\vec{x}_{c}$ along the panel normal vector $\bar{n}_{c}$ (see equation (3.1)) onto the surface. To determine the points $\vec{x}_{e}$ the following algorithm is used:

Input: Collocation point $\vec{x}_{c}$, normal vector $\vec{n}_{c}$ at $\vec{x}_{c}$

Output: Point $\vec{x}_{e}$ on the surface of the ellipsoid along $\bar{n}_{c}$

Initial guess: $\vec{x}_{e}:=\vec{x}_{c}$

$$
\begin{aligned}
& \text { repeat } \\
& \begin{array}{c}
\Delta \ell=-\psi\left(\vec{x}_{e}\right) / \nabla \psi\left(\vec{x}_{e}\right) \cdot \bar{n}_{c} \\
\vec{x}_{e}:=\vec{x}_{e}+\Delta \ell \bar{n}_{c}
\end{array} \\
& \text { until }|\Delta \ell|<\varepsilon
\end{aligned}
$$

Note that for a projection normal to the ellipsoid surface, the algorithm can be easily adapted by updating $\bar{n}_{c}$ with a new normal vector from equation (G.2) each iteration and projecting the collocation point in the direction of this normal vector. 


\section{G.2 Ellipsoid in Uniform Onset Flow}

For ellipsoidal bodies in uniform onset flow analytical potential flow solutions exist (see Durand [33], Lamb [69], Milne-Thomson [75]). The perturbation velocity potential on the surface of an ellipsoid for a general uniform parallel onset flow $\vec{u}_{\infty}=\left(u_{\infty}, v_{\infty}, w_{\infty}\right)^{\mathrm{T}}$, is given by

$$
\varphi\left(\vec{x}_{e}\right)=u_{\infty} x_{e} \frac{\alpha_{0}}{2-\alpha_{0}}+v_{\infty} y_{e} \frac{\beta_{0}}{2-\beta_{0}}+w_{\infty} z_{e} \frac{\gamma_{0}}{2-\gamma_{0}},
$$

and is linear in the three ellipsoid coordinates. The dimensionless coefficients $\alpha_{0}, \beta_{0}$, and $\gamma_{0}$ are

$$
\begin{aligned}
& \alpha_{0}=a b c \int_{0}^{\infty} \frac{1}{\left(a^{2}+\lambda\right) \Delta_{\lambda}} d \lambda, \\
& \beta_{0}=a b c \int_{0}^{\infty} \frac{1}{\left(b^{2}+\lambda\right) \Delta_{\lambda}} d \lambda, \\
& \gamma_{0}=a b c \int_{0}^{\infty} \frac{1}{\left(c^{2}+\lambda\right) \Delta_{\lambda}} d \lambda,
\end{aligned}
$$

in which we used the shorthand notation

$$
\Delta_{\lambda}=\sqrt{\left(a^{2}+\lambda\right)\left(b^{2}+\lambda\right)\left(c^{2}+\lambda\right)} .
$$

These coefficients can be expressed in terms of elliptic integrals. However, above integrals are regular and can also be numerically evaluated up to arbitrary precision given a specific choice of semi-axes $(a, b, c)$. Notice that the sum of the three integrals can be evaluated easily to yield

$$
\alpha_{0}+\beta_{0}+\gamma_{0}=2 .
$$

In table G. 1 the values of $\alpha_{0}, \beta_{0}$, and $\gamma_{0}$ for some combinations of semi-axes $(a, b, c)$ are given. Note that for the sphere $a=b=c=1$ the exact value for the integrals $\alpha_{0}=\beta_{0}=\gamma_{0}=2 / 3$ is reproduced by the numerical evaluation.

For the total potential on the surface of the ellipsoid we can write

$$
\Phi\left(\vec{x}_{e}\right)=\vec{u}_{\infty} \cdot \vec{x}_{e}+\varphi\left(\vec{x}_{e}\right)=\frac{2 u_{\infty}}{2-\alpha_{0}} x_{e}+\frac{2 v_{\infty}}{2-\beta_{0}} y_{e}+\frac{2 w_{\infty}}{2-\gamma_{0}} z_{e} .
$$

The velocity vector component tangential to the surface of the ellipsoid can now be determined from the surface gradient of the total potential (G.10). 
We avoid the introduction of surface metrics to determine the surface gradient directly by first extending the velocity potential that is valid on the surface of the ellipsoid only into the domain, and let $\widetilde{\Phi}(\vec{x})$ be the extended potential. The error introduced by this propagation away from the surface is inconsequential to the surface tangential velocity (G.11) as the erroneous gradient in normal direction is removed again:

$$
\vec{u}_{t}\left(\vec{x}_{e}\right)=\nabla \widetilde{\Phi}-\left(\nabla \widetilde{\Phi} \cdot \bar{n}_{e}\right) \bar{n}_{e}
$$

where we have for the gradient of the extended total velocity potential

$$
\nabla \widetilde{\Phi}=\left(\frac{2 u_{\infty}}{2-\alpha_{0}}, \frac{2 v_{\infty}}{2-\beta_{0}}, \frac{2 w_{\infty}}{2-\gamma_{0}},\right)^{\mathrm{T}} .
$$

The velocity vector component normal to the surface follows from the boundary condition for the stationary geometry and reads

$$
\vec{u}_{n}\left(\vec{x}_{e}\right)=0 .
$$

Table G.1: Elliptic integral values for some combinations of semi-axes $(a, b, c)$.

\begin{tabular}{rccccc}
\hline$a$ & $b$ & $c$ & $\alpha_{0}$ & $\beta_{0}$ & $\gamma_{0}$ \\
\hline \hline 1 & 1 & 1 & 0.6666666666666666667 & 0.66666666666666666667 & 0.66666666666666666667 \\
4 & 2 & 1 & 0.224700883151007738 & 0.569560963385673906 & 1.205738153463318356 \\
8 & 4 & 1 & 0.131334033030569717 & 0.349125891286244098 & 1.519540075683186185 \\
16 & 4 & 1 & 0.050813762040028079 & 0.378853814490314385 & 1.570332423469657536 \\
32 & 4 & 1 & 0.017731858122161341 & 0.392266000999134472 & 1.590002140878704187 \\
64 & 4 & 1 & 0.005751180068755014 & 0.397413990476061750 & 1.596834829455183237 \\
\hline
\end{tabular}

Figure G.2 shows the exact solutions for the perturbation velocity potential and for the pressure distribution on the surface of an ellipsoid with $(4,2,1)$ semi-axes in an onset flow along the $x$-axis. Local pressure coefficients are determined by the Bernoulli equation with the reference velocity equal to the onset velocity.

\section{G.3 Rotating Ellipsoid in Fluid at Rest}

Following the treatises by Lamb [69], Milne-Thomson [75], and Munk in [33], the perturbation velocity potential of a tri-axial ellipsoid with half axes $(a, b, c)$ in a fluid at rest, rotating around an arbitrarily oriented axis 


\section{G. Tri-Axial Ellipsoids}

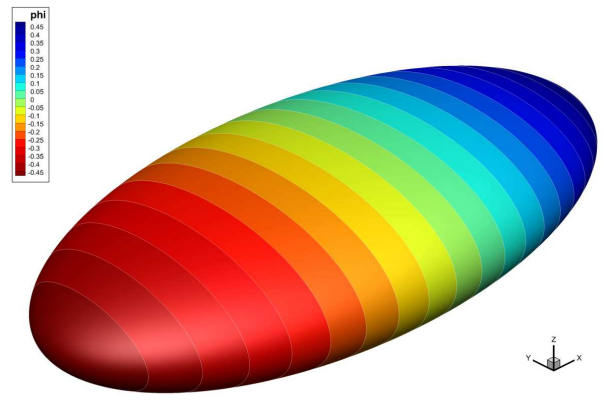

(a) Perturbation velocity potential.

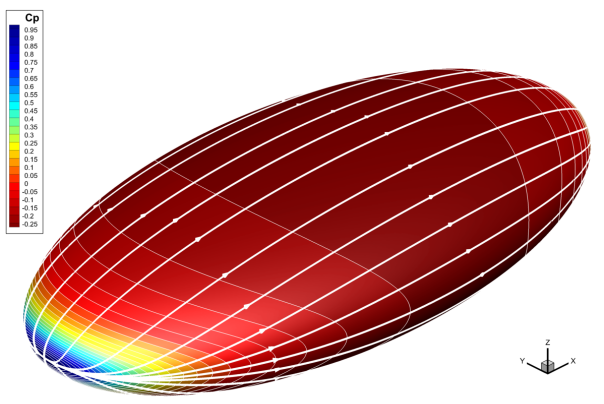

(b) Streamlines and surface pressure distribution.

Figure G.2: Exact solutions for a $(4,2,1)$ semi-axes ellipsoid for onset flow along the $x$-direction, i.e. $\vec{u}_{\infty}=(1,0,0)^{T}$.

$\vec{\Omega}=\left(\Omega_{x}, \Omega_{y}, \Omega_{z}\right)^{\mathrm{T}}$ through its center with angular velocity $|\vec{\Omega}|$, at a point $\vec{x}_{e}$ on the surface of the ellipsoid can be written as

$$
\varphi\left(\vec{x}_{e}\right)=\Omega_{x} y_{e} z_{e} \xi_{0}+\Omega_{y} z_{e} x_{e} \eta_{0}+\Omega_{z} x_{e} y_{e} \zeta_{0}
$$

with the dimensionless coefficients $\xi_{0}, \eta_{0}$, and $\zeta_{0}$ defined as ${ }^{1}$

$$
\begin{aligned}
& \xi_{0}=\frac{-a b c\left(b^{2}-c^{2}\right)^{2}}{2\left(b^{2}-c^{2}\right)+\left(b^{2}+c^{2}\right)\left(\beta_{0}-\gamma_{0}\right)} \int_{0}^{\infty} \frac{1}{\left(b^{2}+\lambda\right)\left(c^{2}+\lambda\right) \Delta_{\lambda}} d \lambda, \\
& \eta_{0}=\frac{-a b c\left(c^{2}-a^{2}\right)^{2}}{2\left(c^{2}-a^{2}\right)+\left(c^{2}+a^{2}\right)\left(\gamma_{0}-\alpha_{0}\right)} \int_{0}^{\infty} \frac{1}{\left(c^{2}+\lambda\right)\left(a^{2}+\lambda\right) \Delta_{\lambda}} d \lambda, \\
& \zeta_{0}=\frac{-a b c\left(a^{2}-b^{2}\right)^{2}}{2\left(a^{2}-b^{2}\right)+\left(a^{2}+b^{2}\right)\left(\alpha_{0}-\beta_{0}\right)} \int_{0}^{\infty} \frac{1}{\left(a^{2}+\lambda\right)\left(b^{2}+\lambda\right) \Delta_{\lambda}} d \lambda .
\end{aligned}
$$

Above integrals can be expressed in terms of the parameters $\alpha_{0}, \beta_{0}$, and $\gamma_{0}$ from the preceeding section, following Milne-Thomson [75], by noting that for example for equation (G.15) we can write the integrand as partial fractions and use

$$
\frac{1}{b^{2}+\lambda}-\frac{1}{c^{2}+\lambda}=\frac{-\left(b^{2}-c^{2}\right)}{\left(b^{2}+\lambda\right)\left(c^{2}+\lambda\right)}
$$

${ }^{1}$ The coefficients differ in sign with those in Lamb [69], Milne-Thomson [75], and Munk [33] due to an opposite sign in boundary conditions. 
and similar expressions for equations (G.16) and (G.17) by cyclic variation through the length of the half axes $(a, b, c)$. We thus find that

$$
\begin{aligned}
\xi_{0} & =\frac{\left(\beta_{0}-\gamma_{0}\right)\left(b^{2}-c^{2}\right)}{2\left(b^{2}-c^{2}\right)+\left(b^{2}+c^{2}\right)\left(\beta_{0}-\gamma_{0}\right)}, \\
\eta_{0} & =\frac{\left(\gamma_{0}-\alpha_{0}\right)\left(c^{2}-a^{2}\right)}{2\left(c^{2}-a^{2}\right)+\left(c^{2}+a^{2}\right)\left(\gamma_{0}-\alpha_{0}\right)}, \\
\zeta_{0} & =\frac{\left(\alpha_{0}-\beta_{0}\right)\left(a^{2}-b^{2}\right)}{2\left(a^{2}-b^{2}\right)+\left(a^{2}+b^{2}\right)\left(\alpha_{0}-\beta_{0}\right)},
\end{aligned}
$$

and define $0 / 0=0$, so that the perturbation potential for an axisymmetric ellipsoid rotating around its symmetry line is zero. In table G.2 the values of $\xi_{0}, \eta_{0}$, and $\zeta_{0}$ for some combinations of semi-axes $(a, b, c)$ are given.

Table G.2: Elliptic integral values for some combinations of semi-axes $(a, b, c)$.

\begin{tabular}{rrrrrr}
\hline$a$ & $b$ & $c$ & $\xi_{0}$ & $\eta_{0}$ & \multicolumn{1}{c}{$\zeta_{0}$} \\
\hline \hline 1 & 1 & 1 & 0.000000000000000000 & 0.000000000000000000 & 0.000000000000000000 \\
4 & 2 & 1 & -0.676996934726978718 & 1.104575464123506286 & -0.241967476150079956 \\
8 & 4 & 1 & -1.737729807684043291 & 2.445213216077034812 & -0.133042182608398807 \\
16 & 4 & 1 & -1.834010180280781559 & 3.242929752359723435 & -0.201471420199926423 \\
32 & 4 & 1 & -1.863990133169630757 & 3.702457389944820493 & -0.232114365685211036 \\
64 & 4 & 1 & -1.872174962679353822 & 3.898384172901041257 & -0.243986339485978655 \\
\hline
\end{tabular}

The velocity vector component tangential to the surface of the ellipsoid can now be determined from the surface gradient of the perturbation velocity potential. As in the previous section, we first propagate the perturbation velocity potential (G.14) into the domain and denote it by $\widetilde{\varphi}(\vec{x})$. To obtain the tangential velocity the gradient of this extended potential function is determined and the erroneous component in normal direction is removed again:

$$
\vec{u}_{t}\left(\vec{x}_{e}\right)=\nabla \widetilde{\varphi}-\left(\nabla \widetilde{\varphi} \cdot \bar{n}_{e}\right) \bar{n}_{e},
$$

where we have for the gradient of the propagated velocity potential at point $\vec{x}_{e}$ on the surface

$$
\nabla \widetilde{\varphi}=\left(\begin{array}{c}
\Omega_{y} z_{e} \eta_{0}+\Omega_{z} y_{e} \zeta_{0} \\
\Omega_{z} x_{e} \zeta_{0}+\Omega_{x} z_{e} \xi_{0} \\
\Omega_{x} y_{e} \xi_{0}+\Omega_{y} x_{e} \eta_{0}
\end{array}\right) .
$$

The perturbation velocity vector component normal to the surface of the ellipsoid comes from the boundary condition and is equal to the normal 
component of the surface velocity

$$
\vec{u}_{n}\left(\vec{x}_{e}\right)=\left(\left(\vec{\Omega} \times \vec{x}_{e}\right) \cdot \bar{n}_{e}\right) \bar{n}_{e}
$$

Figure G.3 shows the exact solutions for the perturbation velocity potential and pressure distribution for an ellipsoid with $(4,2,1)$ semi-axes rotating around its $z$-axis with $\vec{\Omega}=(0,0,1)^{\mathrm{T}}\left[\mathrm{rad} \cdot \mathrm{s}^{-1}\right]$. Local pressure coefficients are determined with the reference velocity equal to the local surface velocity $^{2}$.

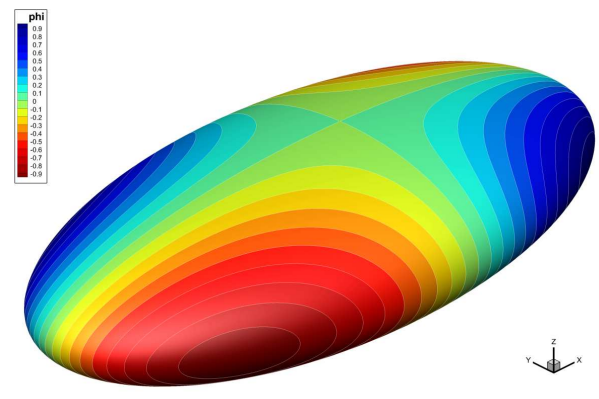

(a) Perturbation velocity potential.

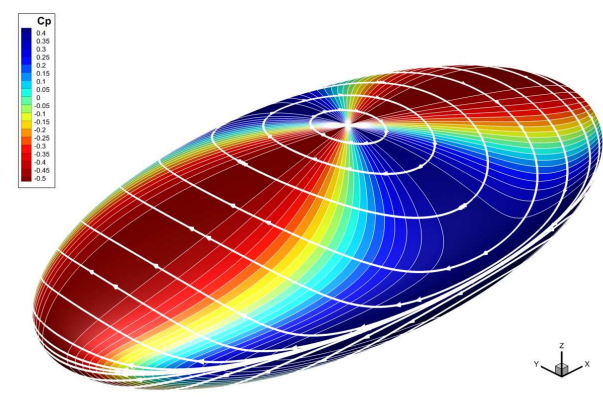

(b) Streamlines and surface pressure distribution.

Figure G.3: Exact solutions for a $(4,2,1)$ semi-axes ellipsoid rotating around the $z$-axis at angular velocity $\vec{\Omega}=(0,0,1)^{T}\left[\mathrm{rad} \cdot \mathrm{s}^{-1}\right]$ in a quiescent environment.

\section{G.4 Flow Solutions for a Two-Dimensional Ellipse}

Starting from the three-dimensional solutions for a tri-axial ellipsoid with semi-axes $(a, b, c)$, the two-dimensional flow solutions for a stationary body with elliptic cross-section in uniform onset flow $\vec{u}_{\infty}$ with angle of attack $\alpha$ and an ellipse in still air rotating about its center with velocity $\Omega_{z}$ are obtained when the dimension of the geometry in $z$-direction $c \rightarrow \infty$ and the solution is taken in the symmetry plane $z=0$. For the rotating ellipse the $z$-axis is the line around which the body rotates. Figure G.4 shows the geometric definitions used.

\footnotetext{
${ }^{2}$ For points where the axis of rotation intersects the ellipsoid surface the local surface velocity is zero and the pressure coefficient is not defined.
} 


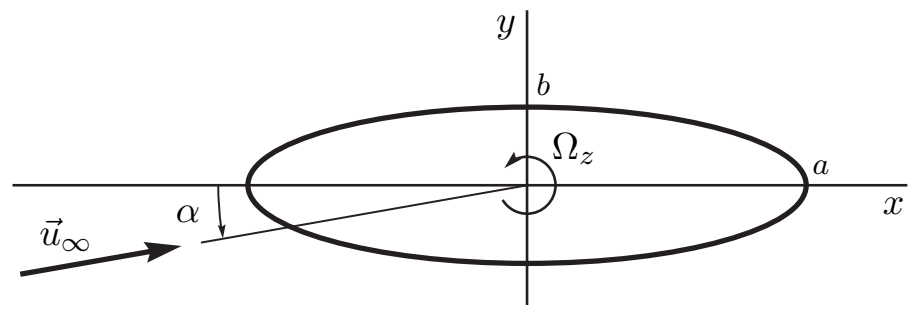

Figure G.4: Definitions for an ellipse with semi-axes $(a, b)$ in a uniform onset flow $\vec{u}_{\infty}$ with angle of attack $\alpha$ and rotating about its center with velocity $\Omega_{z}$.

\section{D Ellipse in Uniform Onset Flow}

For the elliptic integral for $\gamma_{0}$ in equation (G.7) and for the ratio of $\alpha_{0}$ and $\beta_{0}$ in equations (G.5) and (G.6) it can be shown (see reference [82]) that

$$
\begin{aligned}
& \lim _{c \rightarrow \infty} \gamma_{0}=0, \\
& \lim _{c \rightarrow \infty} \frac{\alpha_{0}}{\beta_{0}}=\frac{b}{a} .
\end{aligned}
$$

These limits combined with identity (G.9) result in

$$
\begin{aligned}
\alpha_{0} & =\frac{2 b}{a+b}, \\
\beta_{0} & =\frac{2 a}{a+b} .
\end{aligned}
$$

For the 2D perturbation potential at the surface of the ellipse we find that

$$
\varphi\left(\vec{x}_{e}\right)=u_{\infty} x_{e} \frac{b}{a}+v_{\infty} y_{e} \frac{a}{b},
$$

which gives for the total potential on the surface of the elliptic body

$$
\Phi\left(\vec{x}_{e}\right)=\vec{u}_{\infty} \cdot \vec{x}_{e}+\varphi\left(\vec{x}_{e}\right)=u_{\infty} x_{e}\left(1+\frac{b}{a}\right)+v_{\infty} y_{e}\left(1+\frac{a}{b}\right) .
$$

We extend this total potential from the surface of the ellipse into the flow domain and find for the gradient of the extended total potential $\widetilde{\Phi}(\vec{x})$ that

$$
\nabla \widetilde{\Phi}=\left(u_{\infty} \frac{a+b}{a}, v_{\infty} \frac{a+b}{b}\right)^{\mathrm{T}} .
$$

Using equations (G.2) and (G.3) for the unit normal vector and expression (G.11) for the tangential velocity at the surface we can now obtain 
an explicit expression for the flow solution in the symmetry plane $z=0$ for a stationary $2 \mathrm{D}$ elliptic body with semi-axes $(a, b)$ in uniform onset flow $\left(u_{\infty}, v_{\infty}\right)^{\mathrm{T}}$. After some manipulations we find for the total velocity tangential at point $\left(x_{e}, y_{e}\right)^{\mathrm{T}}$ at the surface

$$
\vec{u}_{t}\left(\vec{x}_{e}\right)=\left(u_{\infty} \frac{a+b}{a}, v_{\infty} \frac{a+b}{b}\right)^{\mathrm{T}}-\kappa_{\alpha}\left(\frac{x_{e}}{a^{2}}, \frac{y_{e}}{b^{2}}\right)^{\mathrm{T}}
$$

where

$$
\kappa_{\alpha}=\frac{u_{\infty} x_{e}(a+b) a b^{4}+v_{\infty} y_{e}(a+b) a^{4} b}{x_{e}^{2} b^{4}+y_{e}^{2} a^{4}} .
$$

The velocity vector component in surface normal direction is determined by the boundary condition and equals

$$
\vec{u}_{n}\left(\vec{x}_{e}\right)=\overrightarrow{0} .
$$

An example result for an ellipse with semi-axes $(4,1)$ at $0^{\circ}$ and $10^{\circ}$ angle of attack in a unit onset flow is shown in Figure G.5.

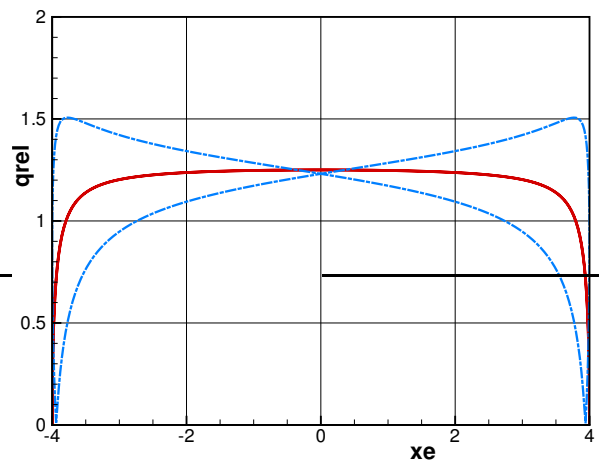

(a) Velocity magnitudes for $\alpha=0^{\circ}$ and $\alpha=10^{\circ}$.

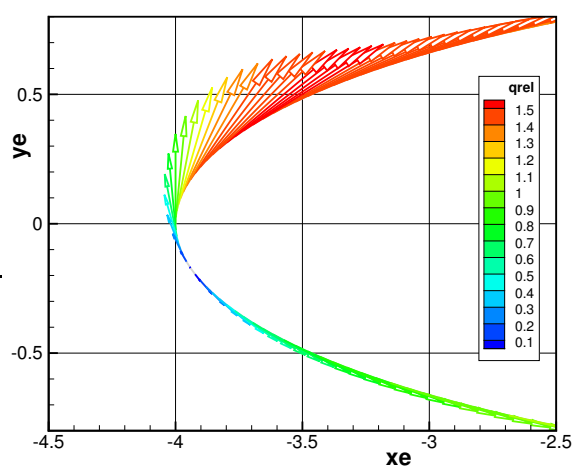

(b) Velocity vectors for $\alpha=10^{\circ}$.

Figure G.5: Velocity distributions for an ellipse with semi-axes $(4,1)$ in a unit onset flow at $0^{\circ}$ and $10^{\circ}$ angle of attack.

\section{D Rotating Ellipse in Fluid at Rest}

From equations (G.26) and (G.27) for $\alpha_{0}$ and $\beta_{0}$ we can derive an expression for the coefficient $\zeta_{0}$ in equation (G.20) that is associated with the ellipse rotating around the $z$-axis with angular velocity $\Omega_{z}$ and obtain

$$
\zeta_{0}=\frac{b^{2}-a^{2}}{2 a b} \text {. }
$$


For the perturbation potential at the surface of the rotating ellipse we can now write

$$
\varphi\left(\vec{x}_{e}\right)=\Omega_{z} x_{e} y_{e} \zeta_{0}=\Omega_{z} x_{e} y_{e} \frac{b^{2}-a^{2}}{2 a b} .
$$

We extend this perturbation potential into the domain and write for the gradient in $\vec{x}_{e}$

$$
\nabla \widetilde{\varphi}=\Omega_{z} \frac{b^{2}-a^{2}}{2 a b}\left(y_{e}, x_{e}\right)^{\mathrm{T}} .
$$

After some manipulations we find for the total tangential velocity at point $\left(x_{e}, y_{e}\right)^{\mathrm{T}}$ at the surface

$$
\vec{u}_{t}\left(\vec{x}_{e}\right)=\Omega_{z} \frac{b^{2}-a^{2}}{2 a b}\left(y_{e}, x_{e}\right)^{\mathrm{T}}-\Omega_{z} \kappa_{\Omega}\left(\frac{x_{e}}{a^{2}}, \frac{y_{e}}{b^{2}}\right)^{\mathrm{T}},
$$

where

$$
\kappa_{\Omega}=\frac{a b\left(b^{4}-a^{4}\right) x_{e} y_{e}}{2 x_{e}^{2} b^{4}+2 y_{e}^{2} a^{4}} .
$$

The normal velocity component is determined by the motion of the body as expressed in equation (G.23). After substitution of the relevant parameters for $z=0$ we find the expression

$$
\vec{u}_{n}\left(\vec{x}_{e}\right)=\Omega_{z} \frac{\left(a^{2}-b^{2}\right) a^{2} b^{2} x_{e} y_{e}}{x_{e}^{2} b^{4}+y_{e}^{2} a^{4}}\left(\frac{x_{e}}{a^{2}}, \frac{y_{e}}{b^{2}}\right)^{\mathrm{T}} .
$$

Figure G.6 shows the result for an ellipse with semi-axes $(4,1)$ rotating around the $z$-axis with $10^{\circ}$ per second in a fluid at rest. The relative velocity vectors are from the viewpoint of an observer on the surface and obtained from the absolute velocity vectors by subtracting the local velocity of the surface due to the rotation. 


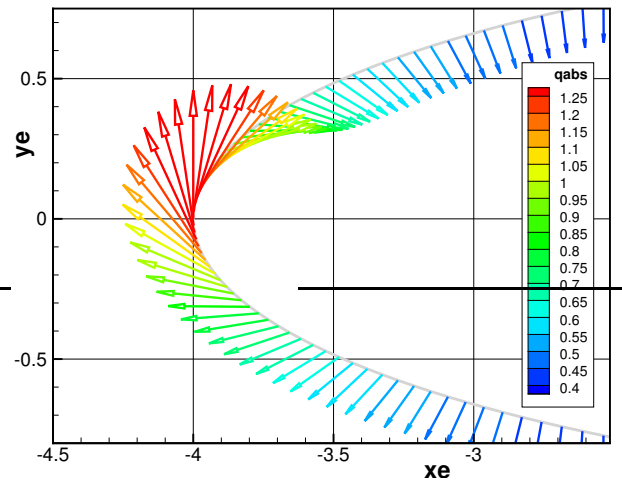

(a) Absolute velocity.

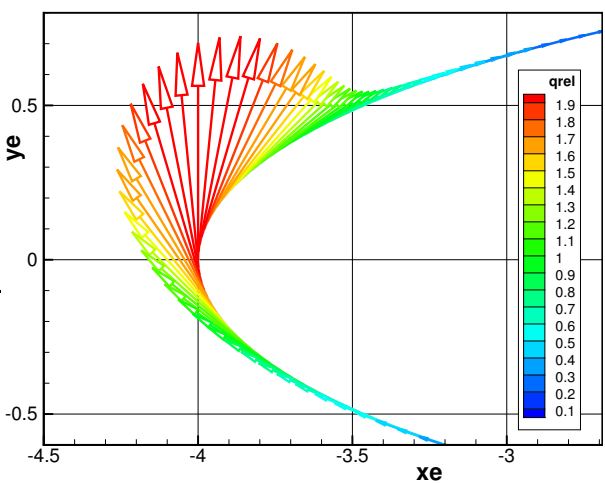

(b) Relative velocity.

Figure G.6: Absolute and relative velocity vectors for an ellipse with semi-axes $(4,1)$ rotating around its center in anti-clockwise direction with $10^{\circ}$ per second in a fluid at rest. 


\section{Acknowledgements}

First and foremost, I would like to express my gratitude to my promotores Harry Hoeijmakers and Kees Venner. Their patience, friendship, attention to detail, and love for the research field of fluid dynamics was for me a lifeline in an ocean of indifference. The group Engineering Fluid Dynamics at the University of Twente is a very nice collaborative environment in which research is valued and all efforts are appreciated: thank you both for that!

At ECN I was privileged to work with the late Danny Winkelaar who passed away unexpectedly in 2007. He set an example with his attention to detail, his great sense of fairness and loyalty, and his uncompromising stance in matters of principle. Danny also introduced me to the $\mathrm{AT}_{\mathrm{E}} \mathrm{X}$ typesetting system and encouraged me to pursue the present $\mathrm{PhD}$ research in my spare time.

I want to thank Hüseyin Özdemir and Sander van der Pijl who grew from being "just colleagues" at ECN to much more. It is always nice to have "brothers in arms" with lots of humor, an unconventional look upon the world, a very sharp mind, and a healthy distrust of imposed authority. Thank you for all your suggestions and encouragements.

I also want to thank the rest of my former colleagues at ECN with whom I shared a passion for wind turbine technology, with an explicit mention for Herman Snel, Edwin Bot, and Johan Peeringa. I have appreciated our conversations very much. I would also like to thank Özlem Çeyhan for generating the initial grid for the MEXICO rotor.

I would like to thank NLR, specifically Sebastiaan ten Pas for sharing the results of the ENSOLV simulations and Jaap van Muijden and Kees Wijnberg who made the cooperation possible.

I also want to thank my fellow researchers in the group Engineering Fluid Dynamics at the University of Twente. I was for several months "on sabbatical" in Twente and during that time I felt myself at home and a member of the team: Koen van Andel, Brenda Benders, Wouter den Breeijen, Hugo Buffy, Dirk van Eijkeren, Ellen van Emden, Giorgio Giangaspero, Rob Hagmeier, Mico Hirschberg, Frans de Jongh, Sietse Jongsma, Faraz Khatami, Bart Konijn, Niels Kruyt, Anjenet Mettivier Meyer, Paul Niël, Ellen Norde, Jesse Slot, Herman Stobbe, Hein de Vries, and Edwin van der Weide. 


\section{About the Author}

Arne van Garrel was born on February $18^{\text {th }} 1965$ in Amsterdam, The Netherlands. He had his high school education at Zaanlands Lyceum where he obtained his Atheneum diploma in 1983. After high school he started his study at Delft University of Technology, faculty of Aerospace Engineering, where he specialized in the area of low speed aerodynamics in the group of Prof. J.L. van Ingen. In cooperation with Ir. L.M.M. Boermans he designed a laminar airfoil for high-performance sailplanes of Alexander Schleicher Segelflugzeugbau [14].

His master thesis research was finalized in 1990 at Fokker Aircraft concerning a transonic wing design for an 80-seat aircraft. After receiving his MSc. degree he continued working in the "Numerical Aerodynamics" group of Fokker Aircraft where he shared a room with Gert-Jan Schipholt, a panel method specialist from NLR who made a career switch to Fokker. Arne's main activities were in aerodynamic design projects, CFD tool development, and maintaining contact with NLR on their latest developments in panel method technology.

After the bankruptcy of Fokker in 1996 he had an interim job at IBM with specialization on Object-Oriented Programming which was followed in 1998 by a research position at ECN in the group Analysis \& Design of the Wind Energy unit. There he worked on prediction methods for wind turbine aerodynamics, specifically panel methods and free vortex wake methods. During his time at ECN he continued working together with Jack van Hengst, the former head of the Aerodynamics \& Aeroelasticity Department at Fokker Aircraft, on CFD analyses for the Dornier Do 728 commercial airliner and on the aerodynamic wing design of the Grob G180 SPn business jet.

In 2015, after 17 years of research into wind turbine aerodynamics, he left ECN to focus on his PhD research on multilevel panel method technology for wind turbine applications. In cooperation with Prof. C.H. Venner he started lecturing the course "Wind Energy" at the University of Twente in the same year. 